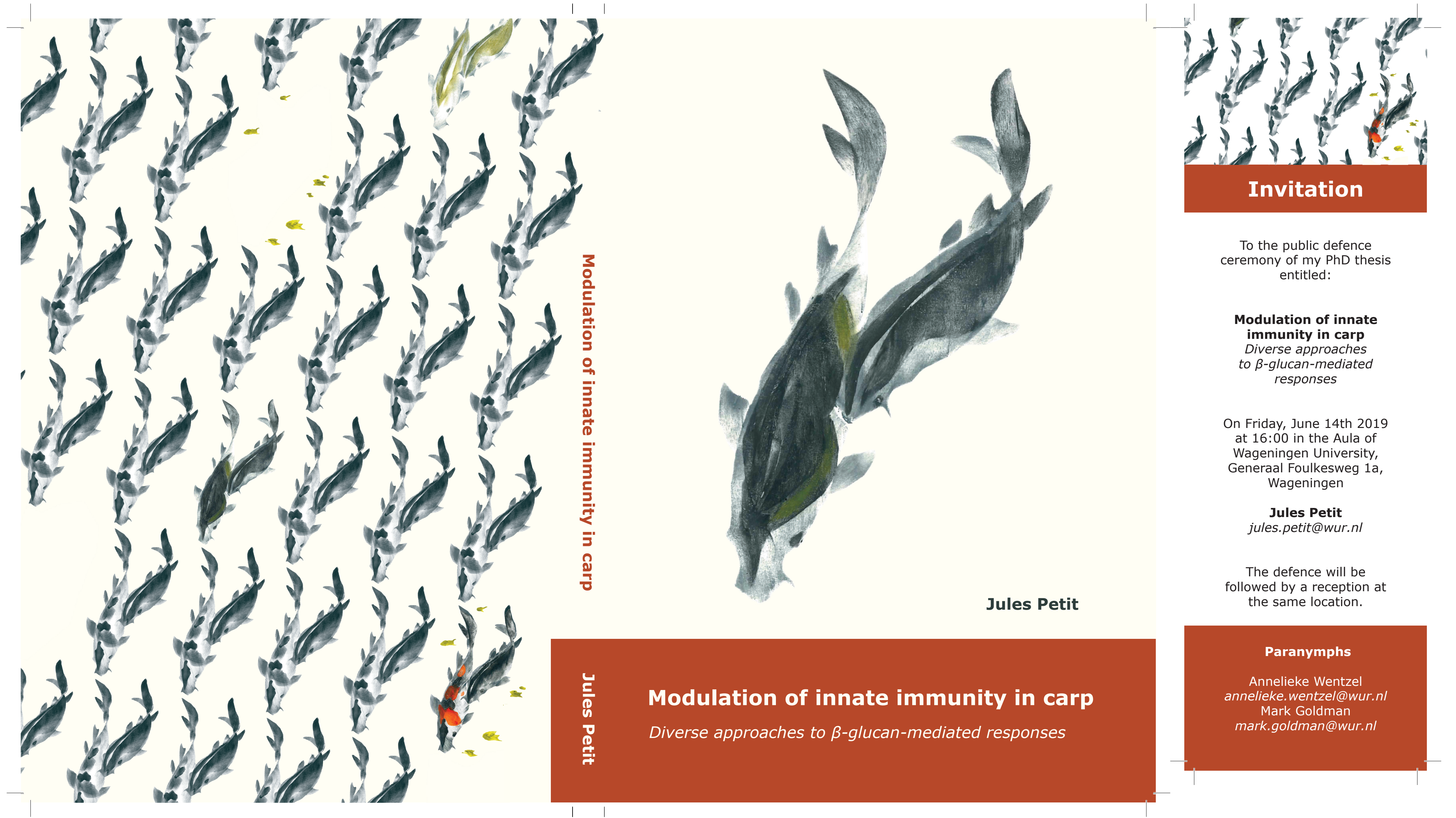




\section{Propositions}

1. Trained immunity is a conserved feature of common carp macrophages (this thesis).

2. $\beta$-glucans activate a Dectin-1-like signalling pathway in carp macrophages (this thesis).

3. If an increase in ploidy can improve tastiness of strawberries (inspired by Edger et al., 2019; Nature Genetics), there is hope for the tetraploid common carp.

4. Social networking can never be about connecting if not interrupted by frequent and conscious disconnecting.

5. Although an inborn enthusiastic and positive attitude can be perceived as naive, it is of great advantage to one's scientific career.

6. Since time has become our most valuable asset, we should value more the cost of other people's willingness to spend time with us.

Propositions belonging to the thesis, entitled

Modulation of innate immunity in carp

Diverse approaches to $\beta$-glucan-mediated responses

Jules Petit

Wageningen, 14 June 2019 


\section{Modulation of innate immunity in carp \\ Diverse approaches to $\beta$-glucan-mediated responses}

Jules Petit 


\section{Thesis committee}

\section{Promotors}

Prof. Dr G.F. Wiegertjes

Professor of Aquaculture and Fisheries

Wageningen University \& Research

Dr M. Forlenza

Associate professor, Cell Biology and Immunology group

Wageningen University \& Research

\section{Other members}

Prof. Dr D.R. Barreda, University of Alberta, Canada

Prof. Dr P. Boudinot, Institut national de la recherche agronomique (INRA),

France

Prof. Dr M. Kleerebezem, Wageningen University \& Research

Prof. Dr H.A. Schols, Wageningen University \& Research

This research was conducted under the auspices of the Graduate School Wageningen Institute of Animal Sciences. 


\title{
Modulation of innate immunity in carp Diverse approaches to $\beta$-glucan-mediated responses
}

\author{
Jules Petit
}

\section{Thesis}

submitted in fulfilment of the requirements for the degree of doctor at Wageningen University

by the authority of the Rector Magnificus,

Prof. Dr A.P.J. Mol, in the presence of the

Thesis Committee appointed by the Academic Board to be defended in public on Friday 14 June 2019 at 4 p.m. in the Aula. 
J. Petit

Modulation of innate immunity in carp - Diverse approaches to $\beta$-glucanmediated responses, 202 pages.

PhD thesis, Wageningen University, Wageningen, the Netherlands (2019) With references, with summaries in English and in Dutch

ISBN: $978-94-6343-946-6$

DOI: https://doi.org/10.18174/475568 


\section{Table of contents}

$\begin{array}{lll}\text { Chapter } 1 & \text { General introduction } 9\end{array}$

Chapter 2 Long-lived effects of administering $\beta$-glucans: p. 23 indications for trained immunity in fish

Chapter 3 Evidence of trained immunity in teleost fish: $\quad 4$ conserved features in carp macrophages

Chapter $4 \quad \beta$-glucan immuno-modulation in common carp $\quad$ p. 61 intestine: a role for microbiota and its metabolites

Chapter 5 Genomic and transcriptomic approaches to study p. 89 immunology in cyprinids: What is next?

Chapter 6 Studies into $\beta$-glucan recognition in fish suggests p. 115 a key role for the C-type lectin pathway

$\begin{array}{lll}\text { Chapter } 7 & \text { General discussion } & \text { p. } 139\end{array}$

$\begin{array}{ll}\text { R } & \text { References } 155\end{array}$

$\begin{array}{ll}\text { S Summaries } & \text { p. } 183\end{array}$

$\begin{array}{lll}\text { A } & \text { About the author } 193\end{array}$ 




\section{NO Waste: working on a circular economy}

The São Paulo Research Foundation (FAPESP) and The Netherlands Organisation for Scientific Research (NWO) agreed to finance a scientific collaboration aiming at the valorisation of $\beta$-glucan, a common 'waste' product from bioethanol production, as immuno-stimulant for use in aquaculture. This was aimed not only at strengthening the cooperation between the State of São Paulo in Brazil and the Netherlands, but is a subject well in frame with the Bio-based Economy research theme formulated by these two large research organisations.

In 1975, the Brazilian government launched a nationwide program to reduce the use of fossil fuels for automobiles, in favour of bio-ethanol. Currently in Brazil, approximately $40 \%$ of the traditional gasoline consumption is replaced by bioethanol produced from sugar cane $(1,2)$. The main industrial route of bio-ethanol production is through the fermentation of extracted sugar from sugar cane by Saccharomyces cerevisiae yeast (3). While the extracted sugar is used to produce ethanol, some of the left-over residues can be used as a source of renewable energy. A large portion of yeast cells used during the fermentation process, up to $90-95 \%$, is recycled in the Brazilian ethanol industry, which results in yeast cells being reused at minimum twice a day for 200 - 250 days (4). A major constituent of yeast cell walls are $\beta$-glucans, comprising over $50 \%$ of the cell wall dry mass weight $(5,6)$. These $\beta$-glucans can be isolated and be used in feed for companion animals and for production animals.

The possibility to use waste products such as $\beta$-glucans from yeast cell walls as feed supplements can further improve waste reduction. This formed the rationale for a scientific collaboration between two university partners; the Cell Biology and Immunology (CBI) group at Wageningen University \& Research and the Centro de Aquicultura da Unesp (Caunesp) at Universidade Estadual Paulista (UNESP), Campus de Jaboticabal, and the research unit of a commercial partner in Brazil (Biorigin). The scientific rationale for the project entitled "Use of branched 1,3/1,6 $\beta$-glucan, MacroGard ${ }^{\circledR}$, a waste product of the production of sugar and ethanol from baker's yeast, to stimulate the innate immune system of farmed fish (short name NOWASTE)" took shape after earlier observations on the immunomodulating ability of the $\beta$-glucan product, as published in two PhD theses of the Cell Biology and Immunology group $(7,8)$.

The aim of the NOWASTE project was to establish optimal protocols for the valorisation of $\beta$-glucan 'waste' from bioethanol production as immuno-stimulant for use in aquaculture, using $\beta$-glucans in the strategic improvement of fish health. Within the NOWASTE project, the CBI group was responsible for the characterization of molecular mechanisms underlying $\beta$-glucan immunomodulatory effects in fish. Furthermore, the CBI group was responsible for provision of training to the Brazilian partners in relevant in vitro assays and for providing a theoretical framework that could support field studies in Brazil. 


\section{Growth and intensification of aquaculture}

The aquaculture sector keeps growing rapidly and, as illustrated by the Food and Agriculture Organisation of the United Nations in their biyearly reports on 'State of World Fisheries and Aquaculture (SOFIA)', the consumption of fish has been growing with average rate of $3.2 \%$ per year since the 1960 s (9). During this period the average growth of the world population was $1.6 \%$ per year, which means that fish consumption per capita has increased. In 2015 , over $40 \%$ of the world population derived at least $20 \%$ of their animal protein intake from fish. With a growing consumption of fish and a growing world population, pressure on fisheries and aquaculture is increasing. In 2016, the total fisheries production accounted for 171 million tons, of which $47 \%$ could be ascribed to aquaculture (9). Almost $60 \%$ of this production could be accounted for by inland finfish production. The current expectation is that in 2020, total aquaculture production will surpass the total capture fisheries and the total aquaculture production for human consumption for 2030 is estimated to be 109 million tons alone.

In Brazil, the aquaculture sector is also growing fast, as illustrated by the tremendous increase from 30.000 tonnes in the 1990 s to over 275.000 tonnes in $2003^{1}$. From 2005 until 2015, the Brazilian aquaculture yield has further grown with a staggering $123 \%$ to 574.000 tonnes per year ${ }^{2}$ and in 2016 the FAO assessed the total aquaculture production of Brazil to be 581.000 tonnes, over $87 \%$ from inland finfish production, with an expected growth towards a total aquaculture production of 1.097 .000 tonnes in 2030 (9).

The intensification of the aquaculture industry accommodated for the growth of the sector and the growing demand for fish, but it came with some drawbacks. Intensification of aquaculture led to increased stress due to environmental factors such as low water quality, high temperature and high fish density (10). Increased stress, lack of adaptation to local infectious agents, increased host contact rates due to high stocking density and intensive monoculture has resulted in a clear increase in disease incidence (11-14) and economic losses (14) over the past decades. The economic losses in the Brazilian aquaculture farms due to diseases were estimated to a total of 84 million US dollars per year (15).

\footnotetext{
${ }^{1}$ As reported by FAO (http://www.fao.org/fishery/countrysector/naso brazil/en, accessed 20-02-2019)

2 As reported by Embrapa on 13-12-2016 (Brazilian Agricultural Research Corporation commissioned by the Brazilian Ministry of Agriculture, Livestock, and Food Supply)
} 


\section{Immuno-modulation of fish through $\beta$-glucan supplementation}

Using $\beta$-glucans to modulate the immune system of farmed fish can be a sustainable solution to reduce losses in the aquaculture sector, especially when derived as waste product. Further, the use of immuno-modulatory $\beta$-glucans to prevent disease and or increase resilience of fish in aquaculture in alternative to the use of antibiotics, might help reduce the detrimental effects of antibiotic spill over into the environment and food chain (16-18). Already in the 90's of the previous century it became evident that dietary supplementation or administration through injection of $\beta$-glucans could resulted in increased bacterial and viral resistance of fish [as reviewed by: (19-21)].

$\beta$-glucans are polysaccharides made by glucose linked by $\beta$-glycosidic bonds. $\beta$ glucans are classified as non-starch polysaccharides (NSPs), a complex group of molecules composed predominantly of linked monomers of hexoses and pentoses, e.g., galactose, glucose, arabinose, xylose and mannose, such as cellulose, hemicellulose (arabinoxylans and $\beta$-glucans) and pectin. Although nutritionists often refer to the adverse effects of NSPs in feed and to their little nutritional value due to their low digestibility, $\beta$-glucans in feed are often considered immunomodulatory (22). $\beta$-glucans are naturally found in the cell wall of yeast, including Candida albicans and yeast of the Saccharomyces genus (including $S$. cerevisiae, or baker's and brewer's yeast) (Figure 1). Furthermore, these polysaccharides can also be found in the cell wall of plants, among others wheat, rye, barley and several Echinacea species, seaweeds, such as Laminaria digitata, fungi and even in the cell wall of several bacterial species $(21,23)$. Depending on the exact source of $\beta$ -

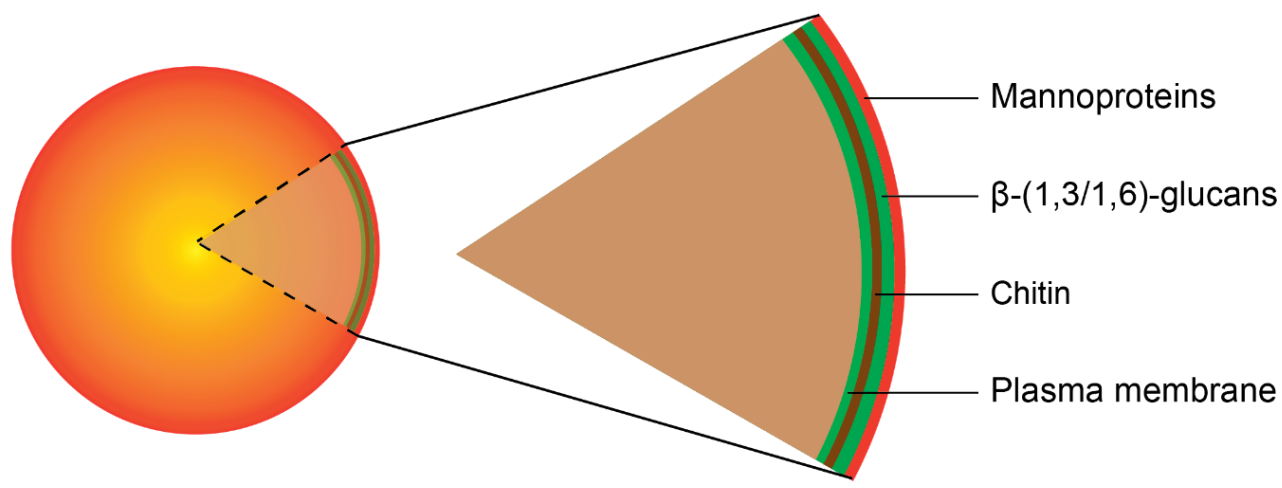

Saccharomyces cerevisiae yeast cell

Figure 1. Schematic overview of a cross-section of the cell wall of $S$. cerevisiae. The outermost layer of the cell wall consists of mannoproteins, followed by a layer with $\beta$ $(1,3 / 1,6)$-glucans, a layer of chitin and the plasma membrane. 
glucan, these polysaccharides exist as a wide variety of structurally diverse molecules with different $\beta$-glycosidic bonds and a linear or branched structure (Figure 2). Plant-derived $\beta$-glucans are often linear with a $\beta-(1,3)-, \beta-(1,4)-$ and $\beta$ - $(1,3 / 1,4)$-conformation, while yeast- and fungi-derived $\beta$-glucans are commonly branched with a $\beta-(1,3 / 1,6)$-conformation. $\beta$-glucan molecules can have a soluble or insoluble character and can have large differences in molecular weight (23). Probably the most widely used $\beta$-glucans in animal husbandry are the high molecular weight $S$. cerevisiae yeast-derived $\beta-(1,3 / 1,6)$-glucans. One of the best studied $\beta$-glucan preparations in aquaculture is MacroGard ${ }^{\circledR}$, a mixture of $\beta$ $(1,3 / 1,6)$-glucans derived from $S$. cerevisiae (24). Although the effects of $\beta$-glucan supplementation in aquaculture have commonly been perceived as positive and beneficial for fish health [reviewed by: (19-21, 24)], no definitive underlying mechanisms explaining the immuno-modulatory effects of $\beta$-glucan in fish had been described at the start of this project.

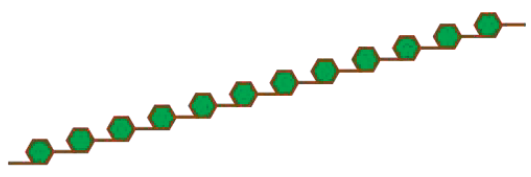

$\beta$-(1,3)-glucan

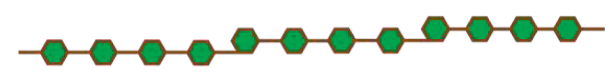

$\beta-(1,3 / 1,4)$-glucan

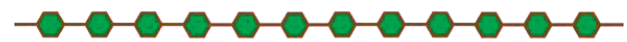

$\beta$-(1,4)-glucan

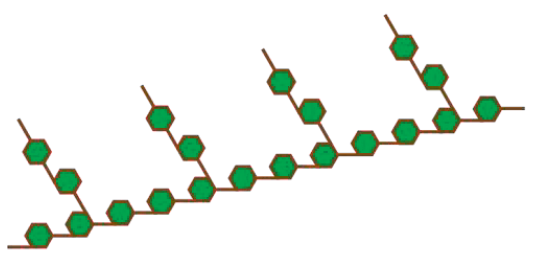

$\beta-(1,3 / 1,6)$-glucan

Figure 2. Schematic representation of different $\boldsymbol{\beta}$-glucan structures. Depending on the position of the linkage, $\beta$-glucans exist in a wide range of different conformations. $\beta$ $(1,3)-, \beta-(1,4)$ - and $\beta-(1,3 / 1,4)$-glucans exist as linear molecules and are often derived from plants or algae. $\beta-(1,3 / 1,6)$-glucans are branched and are often derived from fungi or yeasts.

\section{Direct or indirect effects of $\beta$-glucan supplementation}

There are several ways through which $\beta$-glucans might affect the immune system. Direct effects of $\beta$-glucan supplementation could be induced through sensing $\beta$ glucans as pathogen-associated molecular pattern (PAMP) via direct recognition by pattern recognition receptors (PRRs) on antigen sampling cells such as macrophages or dendritic cells. PRRs are germline-encoded innate immune 
receptors capable of recognizing different PAMPs (25). There are many different classes of PRRs, of which the Toll-like receptors (TLRs), C-type lectin receptors (CLRs) and nucleotide-binding oligomerization domain-like receptors (NLRs) are well known. Different classes of PRRs recognize different classes of PAMPs, and within one class of PRRs many different ligands can be sensed. For example, for the TLR family, TLR4 may sense lipopolysaccharides (LPS) from Gram-negative bacteria whereas TLR3 may sense double stranded RNA. In general, upon binding of a PAMP to a PRR, a downstream signalling cascade will be activated which can finally result in different responses, such as production of inflammatory cytokines or pathogen-induced phagocytosis, the process here referred to as direct effects of $\beta$-glucans (Figure 3A).

A

\section{Direct: \\ PAMP - PRR interaction}
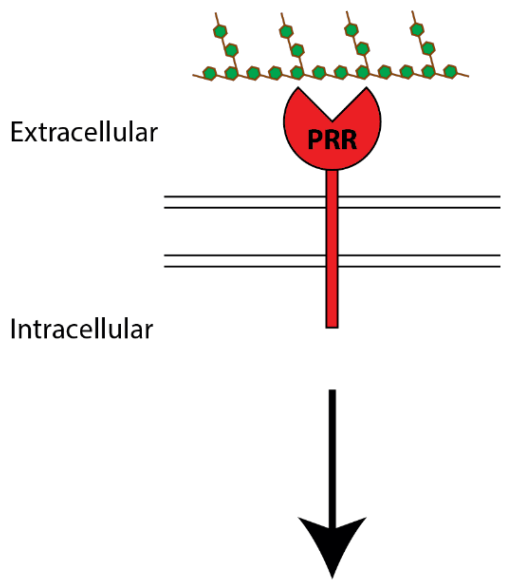

Downstream signalling inducing cytokine production
B

\section{Indirect: \\ Prebiotic effect}

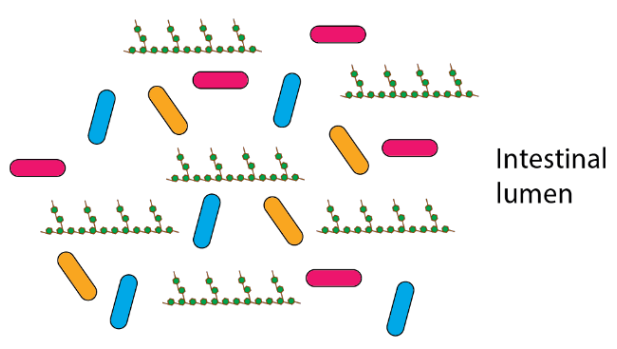

Figure 3. Schematic representation of a PAMP - PRR interaction and possible prebiotic $\boldsymbol{\beta}$-glucan effect. A Branched $\beta$-glucan as pathogen associated molecular pattern (PAMP) binding to a C-type Lectin Receptor (CLR) as pattern recognition receptor (PRR). $\beta$-glucan ligates to the extracellular part of the CLR, which directly activates a signalling pathway leading to various downstream responses, such as the induction of a cytokine response. B branched $\beta$-glucans fermented by intestinal microbes. With respect to indirect prebiotic effects, part of the microbiota is capable of degrading and fermenting $\beta$-glucans present in the intestinal lumen. As a result, fermentation end-products such as short chain fatty acids (SCFAs) can be produced which in turn can affect the host immune system.

Supplementation with $\beta$-glucans can also have indirect effects, often referred to as prebiotic effects. Prebiotics can be defined as "selectively fermented ingredients that allow specific changes, both in the composition and/or activity of the gastrointestinal microflora that confer benefits upon host wellbeing and health" 
(26). Supplementation with $\beta$-glucans could possibly provide a source of nutrient for specific intestinal microbes capable of fermenting these molecules. The fermentation end-products produced by the intestinal microbiota, such as short chain fatty acids (SCFAs), could then influence the host' immune responses, the process here referred to as indirect effects of $\beta$-glucans (Figure $3 \mathrm{~B}$ ).

\section{Direct effects: Dectin-1, the 'main' $\beta$-glucan receptor in mammals}

Direct effects of $\beta$-glucan supplementation could be induced by sensing $\beta$-glucans (PAMP) by a $\beta$-glucan receptor (PRR) on antigen sampling cells. To date, the bestdescribed $\beta$-glucan receptor is Dectin-1, part of C-type Lectin Receptor (CLR) family and also known as C-type Lectin domain Family 7 member A (CLEC7A). This receptor belongs to the $C$-type lectin super family $V, N K$ cell receptors and is a transmembrane receptor with a single carbohydrate recognition domain (CRD) and a cytoplasmic tail containing one immunoreceptor tyrosine-based activation motif (ITAM) (27-29). In mammals, Dectin-1 is predominantly expressed on cells from both the monocyte/macrophage and neutrophil lineages, where it acts as the major receptor for $\beta$-glucans (30). Research has shown that the ligation of $\beta$ - $(1,3)$ glucan to Dectin- 1 is dependent on a $\beta$-glucan binding cleft, formed by the spatial arrangement of three amino acid residues in the carbohydrate binding domain, tryptophan $(\mathrm{W})$ and histidine $(\mathrm{H})$ and tyrosine $(\mathrm{Y})$. The 3-dimensional arrangement of these three amino acid residues in a triangular fashion forms a shallow hydrophobic surface groove, capable of accommodating and binding $\beta$-glucan chains through hydrophobic interactions (31-33). While the three residues are conserved in a WxHxxxxY motif in Dectin-1 in mammals, the same three residues are present in the binding domain of $\beta-(1,3)$-glucan recognition protein (GNBP3) of invertebrates, although not as a conserved motif (34). Dectin-1 signalling is activated following clustering in synapse-like structures, formed within minutes after activation by particulate $\beta$-glucans (35). Following the interaction with $\beta$ glucan, Dectin-1 induces intracellular signalling via the phosphorylation of its ITAM $(6,36)$. Dectin-1 homodimers with phosphorylated ITAM recruit and activate Syk kinase. Activated Syk kinase is responsible for the recruit and activate Syk kinase. Activated Syk kinase is responsible for the phosphorylation of protein-kinase $C \delta$ (PKCD) which in turn phosphorylates the adapter protein Card9 (Caspase recruitment domain-containing protein 9) and the formation of a signalosome with the adapter proteins B cell chronic lymphocytic leukaemia and/or lymphoma 10 (Bcl10) and mucosa-associated lymphoid tissue lymphoma translocation gene 1 (Malt1), (37). The formation Card9-Bcl10-Malt1 signalosome results in activation of NF- $\mathrm{kB}$, leading to an inflammatory profile typical of stimulation with $\beta$-glucans $(38,39)$. Activation of Syk is also required for the induction of reactive oxygen species (ROS) production in response to $\beta$-glucan stimulation (40). Alternatively, Dectin-1 can signal via the Raf-1 kinase pathway, independently of Syk kinase via Ras (Figure 4). Identification of Dectin-1 and/or other C-type lectin super family $V$ 


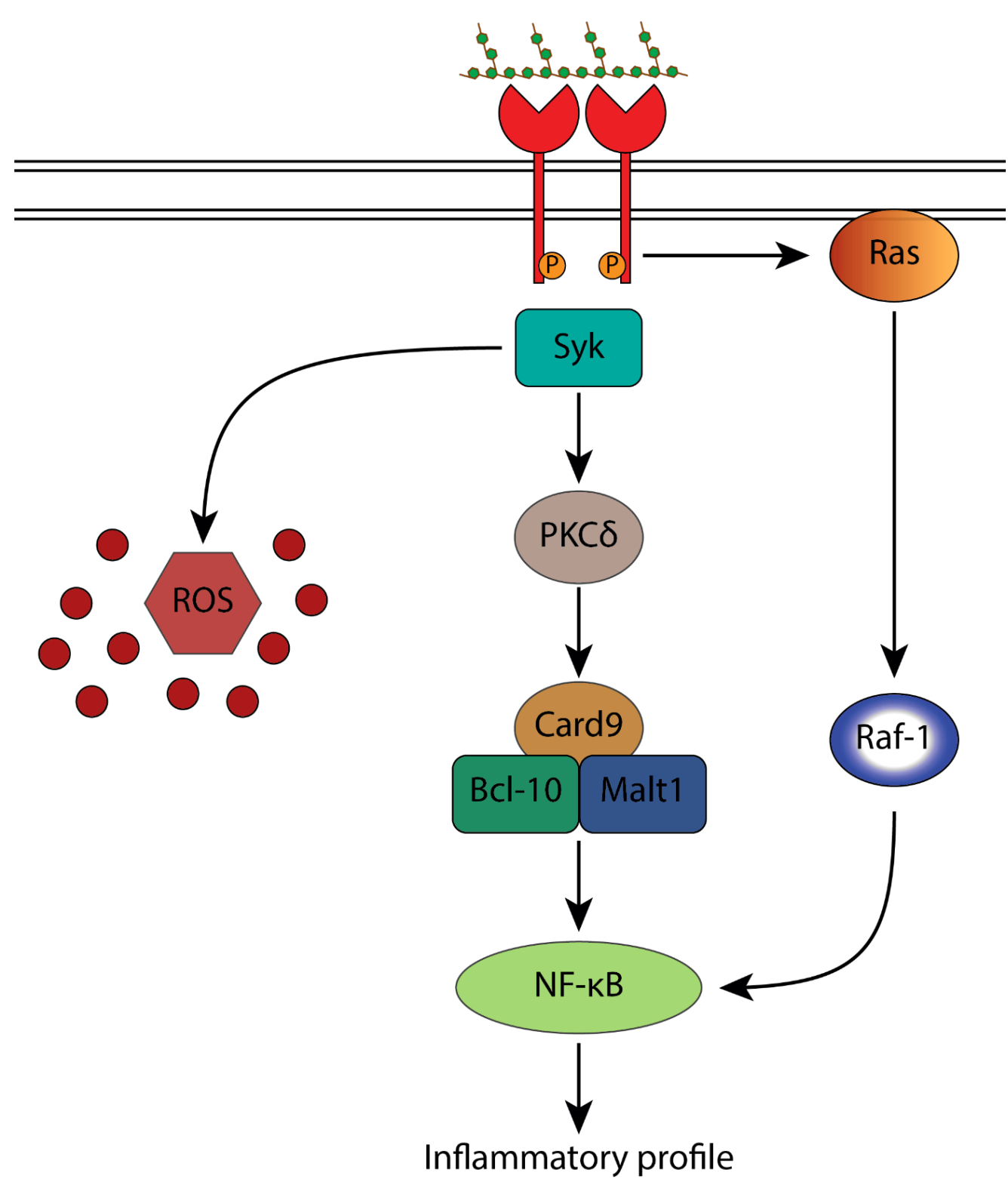

Figure 4. Schematic representation of Dectin-1 signalling. Schematic representation of the signalling of Dectin-1 after ligation with a ligand, e.g. $\beta$-glucans. Dectin-1 homodimers recruit Syk to phosphorylated ITAMs (Orange P), Syk in turn initiates downstream signalling. Syk induces the generation of reactive oxygen species (ROS) and phosphorylates protein-kinase $C \delta$ (PKC $\delta)$. PKC $\delta$ phosphorylates the adapter protein Card9, which facilitates complex formation with the adapter proteins $\mathrm{Bcl}-10$ and Malt1. The formation Card9-Bcl10-Malt1 signalosome results in activation of NF-KB, which results in the inflammatory profile associated with $\beta$-glucan stimulation. Alternatively, Dectin- 1 can activate a Syk independent pathway via Ras, which activates Raf-1 that results in the activation of NF- $\kappa B$. [Adapted from $(6,36)]$. 
NK cell receptors in fish genome assemblies has remained a challenge $(28,41$, 42 ), leaving unanswered the question on the 'main' $\beta$-glucan receptor in fish.

\section{Direct effects: Additional $\beta$-glucan receptors in mammals}

There are several additional recognition mechanisms besides recognition by Dectin- 1 that have been implicated in $\beta$-glucan sensing, i.e. the interplay of Dectin1 with TLRs, the interaction of $\beta$-glucans with Complement Receptor 3 (CR3), the binding of lactosylceramide (LacCer) to $\beta$-glucans and the interaction of Scavenger Receptors (SRs) with $\beta$-glucans (reviewed by: $(19,21,43)$ ). A role for TLR mediated signalling was shown in mice; deficiency in the adaptor protein MyD88 or in TLR2 reduced zymosan-induced IL2 and IL12 production by macrophages, but not the production of ROS $[(44,45)]$. Zymosan is a preparation of $S$. cerevisiae cell wall consisting of not only $\beta$-glucans, but also mannose and chitin (46). A role for CR3 in the response to $\beta$-glucans has also been noted. CR3 is composed of an $a_{m}$ chain, CD11b, and a common $\beta_{2}$ chain, CD18. The $a_{m}$ chain possesses a lectin domain capable of binding $\beta$-glucans $(30,47)$. It was shown that CR3 is the major receptor for $\beta$-glucan particles on human neutrophils and that ligation of the lectinsite primes cells towards iC3b opsonized molecules cytotoxicity $(48,49)$. Another role for sensing $\beta$-glucans can be played by LacCer, a glycosphingolipid with a specificity for the $\beta$-structure in $\beta$-glucans, leading to the induction of macrophage inflammatory protein-2 in mammalian leukocytes $(50,51)$. Finally, SRs have been shown to play a role and in uptake of $\beta$-glucans through competition binding studies (52-54). More recently, a study showed that two SRs, MARCO and CD204, can play a role in the opsonin independent uptake of zymosan in murine macrophages (55). It may be clear that also in fish several 'additional' $\beta$-glucan receptors may be present.

\section{Direct effects: Trained immunity induced by $\beta$ - glucans}

Recently, $\beta$-glucans were connected to a new paradigm in innate immunity. Traditionally, innate immune responses have been viewed as rapid, relatively nonspecific and lacking development of immunological memory. New insights have challenged this view, introducing a novel concept called trained immunity, defined as a heightened non-specific innate immune response to a secondary infection (56, 57), (Figure 5). The initial experiments that lead to the concept of trained immunity showed that $\mathrm{Rag}^{-/-}$mice exposed to a sub-lethal Candida albicans infection were better protected against a subsequent lethal dose of these yeasts, whereas monocyte-deficient mice did not show this increased protection, suggesting a form of memory present in the myeloid compartment. Interestingly, $\beta$-glucans present in the cell wall of $C$. albicans proved crucial for inducing trained immunity. Effects of trained immunity were measured in vivo up to 3 months post primary exposure. 
In vitro re-exposure of human PBMC to C. albicans induced a significant IL6 and TNFa response, even after a resting period of six days in the absence of a stimulus following primary exposure to $\beta$-glucans (58). Subsequently, several studies have further unravelled the underlying mechanisms of trained immunity, showing both metabolic reprogramming (59-63) and epigenetic reprogramming (59, 60, 64-66) following $\beta$-glucan exposure. Before the observation and establishment of the phenomenon trained immunity in humans and mice (67) similar cross-specific protection was observed in invertebrates [reviewed by: (68)] which, typically without T- and B-lymphocytes, can build up a form of immunity, protecting the organism upon a secondary exposure. Given the evolutionary position of teleost fish, as early vertebrates with a fully developed immune system it is likely that trained immune responses should exist also in fish and include receptors for direct sensing of $\beta$-glucan products.

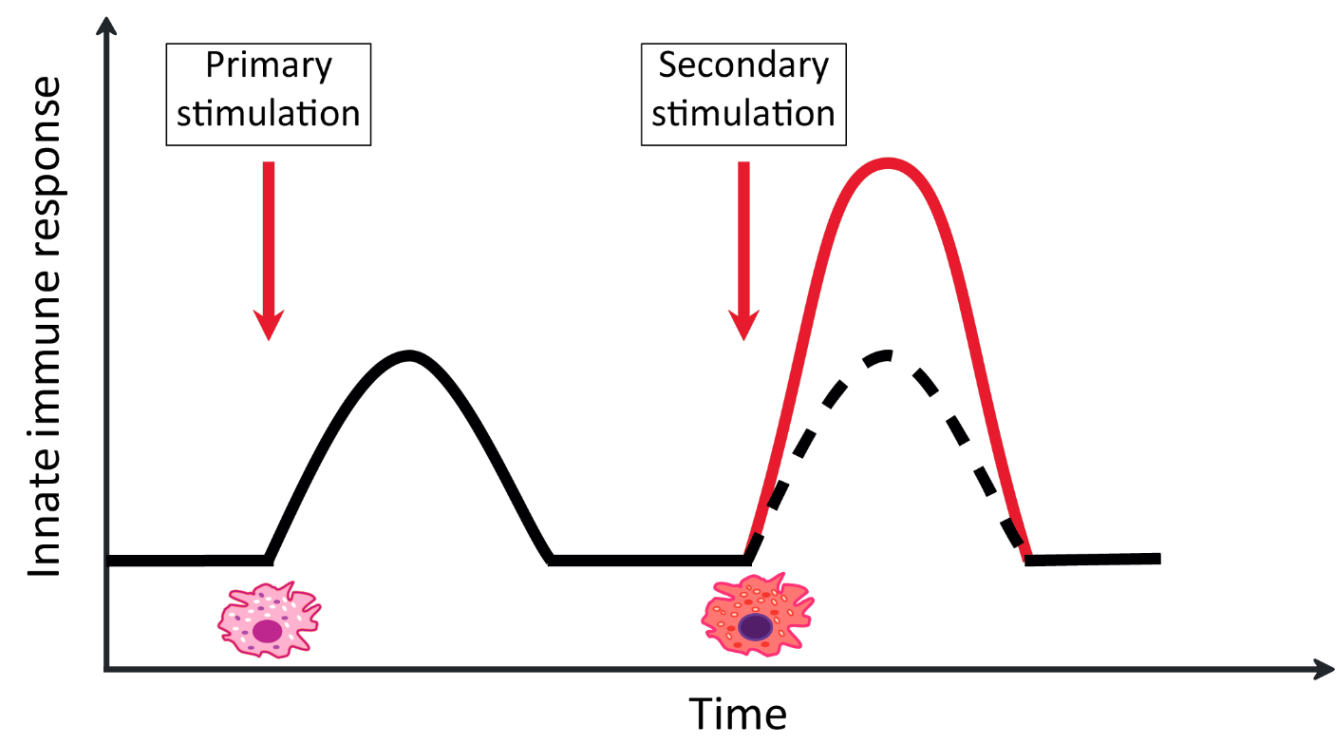

Figure 5. Schematic representation of trained immunity. Black line representing the innate immune response to stimulation, with the dotted black line representing the canonical perspective on the innate immune response to a second stimulation. The red line represents the observed innate immune response ascribed to trained immunity. During the first stimulation, infection or exposure, innate immune cells are reprogrammed, metabolically and epigenetically. Upon second stimulation, infection or exposure, these reprogrammed or 'trained' innate immune cells generate a heightened innate immune response.

\section{Indirect effects: immuno-modulation through intestinal microbiota}

Supplementation with $\beta$-glucans can also have prebiotic effects in the intestinal tract thereby influencing immune responses in an indirect manner. Upon supplementation, when administered orally, $\beta$-glucans may influence the 
composition of the intestinal microbiota or act as a nutrient for particular groups of micro-organisms, thereby causing a shift in intestinal microbiota. Several genera of bacteria common to the gastro-intestinal tract (GIT) are able to degrade and ferment $\beta$-glucans. For example, members of the Bacteroides genus found in the GIT of humans can degrade barley-derived $\beta$-1,4-glucans (69) and fungalderived $\beta-1,6$-glucans (70). Fermentation of $\beta$-glucans by bacteria will result in the production of short chain fatty acids (SCFAs), which in turn affect the host, as they can be sensed by SCFA receptors, G-protein coupled receptor (GPR) 41, GPR43 and GPR109A or directly enter host cells in a receptor-independent manner (7173). Indeed, a study in mice showed that a diet enriched with $\beta$-glucans derived from oat bran led to higher SCFA levels in the caecum (74). Another study that compared the SCFA concentrations in the colon of mice fed five different dietary fibres, observed clear differences in SCFA accumulation and in microbiota composition, even though regulation of epithelial cell's gene expression was comparable between the different dietary fibres (75). In mice production of SCFAs by intestinal microbiota can affect the host and its immune responsiveness. In fact, shifts in the composition of the intestinal microbiota toward more lactic acid bacteria, led to an increase in the IFN- $\beta$ response, induced upon recognition of double stranded RNA shed by these commensal bacteria (76). Of interest, $\beta$ glucans have been shown to stimulate growth of several commensal lactic acid bacteria, at least in humans (77), and preliminary data suggest effects of dietary $\beta$-glucan supplementation on the relative abundance of lactic acid bacteria in the intestinal microbiota of fish $(78,79)$. Thus, prebiotic effects of $\beta$-glucans on the immune system can be classified as indirect when based on shifts in intestinal microbiota, their metabolites, and when based on cellular mechanisms sensing these metabolites or other bacterial products (e.g. double-stranded RNA). No matter what, it may be clear that investigating the prebiotic effects of $\beta$-glucans on the immune system of fish is highly relevant.

\section{Outline of this thesis}

Reports on dietary supplementation with $\beta$-glucan and associated beneficial effects on fish health have been numerous and as a result $\beta$-glucan supplementation is widely applied in aquaculture. Although several studies have investigated the mechanisms of action and searched for potential candidate $\beta$-glucans receptors, no definitive mechanism has been reported that explains the immuno-modulatory effects of $\beta$-glucan in fish. The overall aim of this thesis was to explain the effects of $\beta$-glucans on carp immune responses, studying three different but not necessarily exclusive mechanisms: 1 ) long-lived effects on myeloid cells typical of trained immunity; 2) degradation and fermentation of $\beta$-glucans and associated shifts in composition of the intestinal microbiota and their metabolites; 3 ) regulation of downstream signalling upon $\beta$-glucan recognition in carp macrophages. In this thesis, the three mechanisms are discussed in this order. 
First I discuss the possibility of upgrading waste products from bioethanol production to immuno-stimulants for intensive aquaculture (General introduction, chapter 1 ). Sensing of $\beta$-glucan by an unknown PRR could activate a downstream signalling cascade and pro-inflammatory response, either or not associated with trained immunity, whereas prebiotic effects of $\beta$-glucans could also be indirect when based on shifts in intestinal microbiota.

In chapter $\mathbf{2}$ we review the current literature on $\beta$-glucan research in fish. We sought to update previous studies and reviews in the field and discuss possible mechanisms involved in the immuno-modulatory effects triggered by $\beta$-glucans, including changes in intestinal microbiota, receptor recognition and downstream signalling as well as the relatively new concept of trained immunity. We uncovered several studies with indications for long-lived effects of $\beta$-glucans in fish, but without further experimental evidence, were unable to ascribe these observations to trained immunity.

In chapter 3 we sought to investigate conservation of trained immunity in carp, using an in vitro model based on a known NOD-ligand, peptidoglycan, previously shown to be crucial for the induction of trained immunity in mammals. Following establishment of the model, we investigated the ability of the soluble $\beta$-glucan laminarin to induce trained immunity in carp macrophages, and show that both ligands are suitable training stimuli in carp, effectively expanding the tools available to study trained immunity in teleost fish.

In chapter 4 we set out to investigate the role of intestinal microbiota in the immuno-modulatory effects observed upon dietary $\beta$-glucan supplementation in fish. First, we characterised the normal intestinal microbiota of common carp by 16S rRNA sequencing. Next, in an in vitro batch culture experiment, we analysed the ability of carp intestinal microbiota to ferment $\beta$-glucans, and measured short chain fatty acid (SCFA) profiles. Finally, in vivo $\beta$-glucans supplementation was performed to analyse the effects of $\beta$-glucan on the intestinal microbiota composition and local gene expression.

In chapters $\mathbf{5}$ and $\mathbf{6}$ we investigated the possibilities of using next generation sequencing (NGS) to address our questions related to the mechanisms underlining the immuno-modulatory effects of $\beta$-glucans. In chapter 5 we first reviewed the current genome assemblies in teleost species and summarized current NGS-based studies investigating immune responses in carp and related species. We discuss the complexities and potential pitfalls of working with polyploid species such as common carp. In chapter 6 we applied NGS-based transcriptome analysis to macrophages stimulated with $\beta$-glucans. We tested the hypothesis that immunomodulatory effects of $\beta$-glucan in carp macrophages could be triggered by an unknown member of the C-type Lectin Receptor (CLR) family, different from Dectin-1. Regulation of the C-type lectin receptor signalling pathway suggested at least partial conservation of a $\beta$-glucan recognition cascade via a $C$-type lectin receptor. We conclude with the identification of several candidate receptors for $\beta$ glucans. 
Finally, in chapter 7, the general discussion, I integrate the results of the previous chapters in a larger framework and I address both the limitations and opportunities for further research into $\beta$-glucan supplementation as immuno-stimulant for aquaculture. The findings of this project alongside the interpretation and perspectives offered, pave the way for future research into $\beta$-glucan immunomodulation as well as tailored $\beta$-glucan supplementation strategies in aquaculture. 



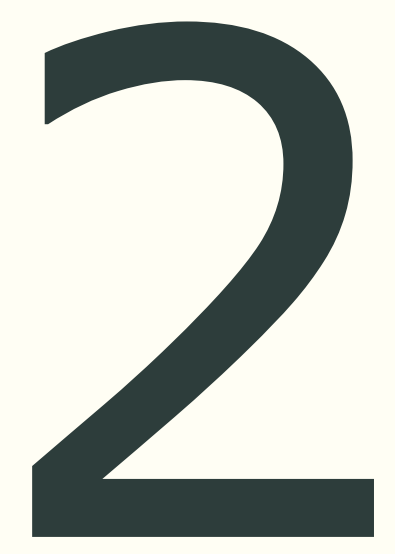

\title{
Long-lived effects of administering $\beta$-glucans: Indications for trained immunity in fish
}

\author{
Jules Petit, Geert F. Wiegertjes
}

Developmental and Comparative Immunology 64 (2016), p. 93-102

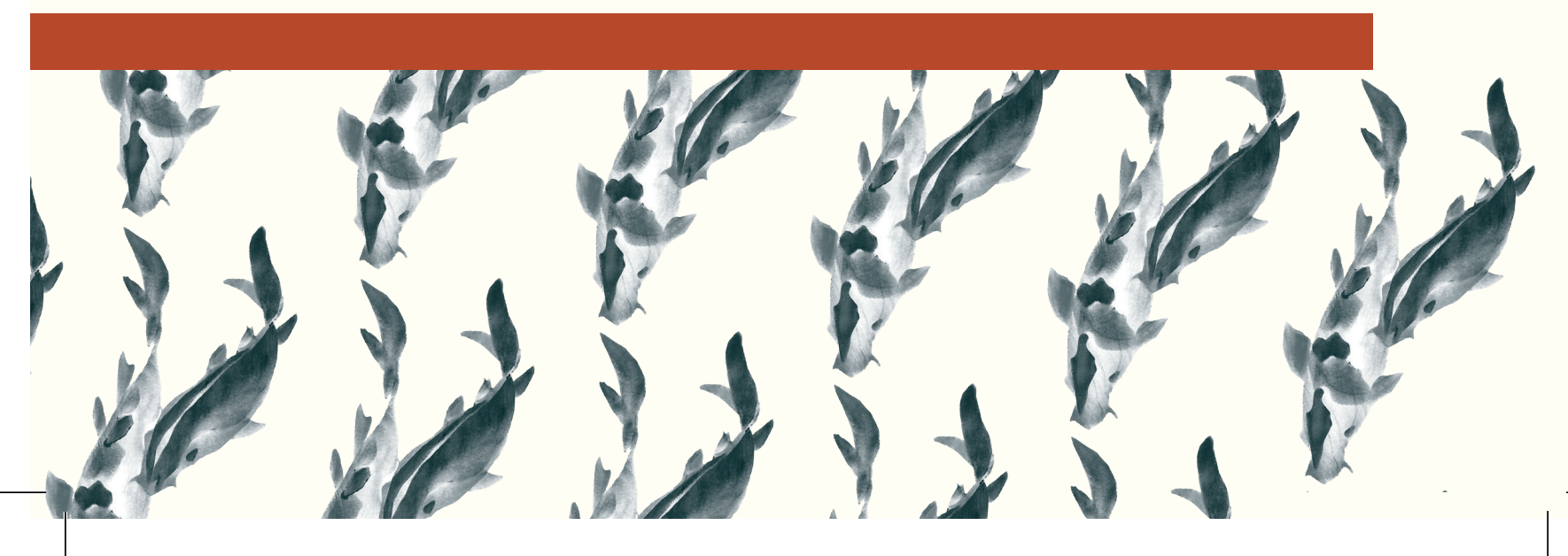




\section{Abstract}

Over the past decades, it has become evident that immune-modulation of fish with $\beta$-glucans, using injection, dietary or even immersion routes of administration, has stimulating but presumed short-lived effects on both intestinal and systemic immunity and can increase protection against a subsequent pathogenic challenge. Although the exact effects can be variable depending on, among others, fish species and administration route, the immune-stimulating effects of $\beta$-glucans on the immune system of fish appear to be universal. This review provides a condensed update of the most recent literature describing the effects of $\beta$-glucans on the teleost fish immune system. We shortly discuss possible mechanisms influencing immune-stimulation by $\beta$-glucans, including microbial composition of the gut, receptor recognition and downstream signalling. Of interest, in mammalian monocytes, $\boldsymbol{\beta}$-glucans are potent inducers of trained immunity. First, we screened the literature for indications of this phenomenon in fish. Criteria that we applied include indications for at least one out of three features considered characteristic of trained immunity; (i) providing protection against a secondary infection in a $\mathrm{T}$ and B-lymphocyte independent manner, (ii) conferring increased resistance upon re-infection and, (iii) relying on key roles for innate immune cell types such as natural killer cells and macrophages. We conclude that several indications exist that support the notion that the innate immune system of teleost fish can be trained. Second, we screened the literature for indications of long-lived effects on innate immunity of fish after administering $\beta$-glucans, a criterion which could help to identify key roles for macrophages on resistance to infection. We discuss whether $\beta$-glucans, as well-known immune-stimulants, are able to train the immune system of fish and argue in favour of further studies designed to specifically investigate this phenomenon in fish. 


\section{Introduction}

Since the 1990's, when immune-stimulation of fish was still under early development, several investigations have suggested that the provision of $\beta$ glucans, either dietary or by supplementary injection, can potentiate the resilience of immune cells $(80-82)$, reduce in vivo stress effects $(81,83)$ and protect teleost fish against subsequent challenges in vivo $(84,85)$. Indeed, provided they are applied as a prophylactic measure, it has become evident that $\beta$-glucans can be a potential immuno-stimulant, suitable for injection and dietary administration, with well-described but short-lived effects on intestinal immunity, systemic immunity and increased protection from a subsequent pathogenic challenge [reviewed by: (19)]. Yet, detailed knowledge of the receptors involved in recognition of $\beta$-glucans and of their downstream signalling is missing for teleosts, leaving obscure whether the observed potentiation should be attributed to direct effects on leukocytes or to indirect effects on, for example, the composition of microbial communities in the gut. Typically, studies investigating the effects of $\beta$-glucans have mostly focussed on relatively short-lived effects, in the order of days up to a few weeks, but recent insights in the field of innate immunity provide indications that $\beta$-glucans could also have effects for a longer period of time, possibly explained by the phenomenon 'trained immunity' (see paragraph 4.3).

$\beta$-glucans are found not only in the cell wall of yeast species, including Candida albicans and yeast of the Saccharomyces genus (S. cerevisiae, or baker's and brewer's yeast), but also in the cell wall of plants including wheat, rye and several Echinacea species, seaweeds, mushrooms and other fungi and even in the cell wall of several bacterial species [reviewed by: (21)]. In fact, $\beta$-glucans comprise a wide variety of structurally diverse molecules (Figure 1 ), which can be short or long, linear or branched and have a soluble or insoluble character but which all have in common that they are polymers comprised of repeating units of glucose, linked by $\beta$-glycosidic bonds $(21,86)$. Although $\beta$-glucans appear to have their immuneactivating capacity in common, there can be clear differences in activity owing to the diversity in structure $(21,87)$. In particular, large molecular weight $\beta$-glucans have stimulatory effects on leukocytes which include the induction of phagocytic, cytotoxic and antimicrobial activities (21). Probably the best-studied and mostapplied $\beta$-glucans are large molecular weight $S$. cerevisiae and $C$. albicans yeastderived $\beta-1,3 / 1,6$-glucans.

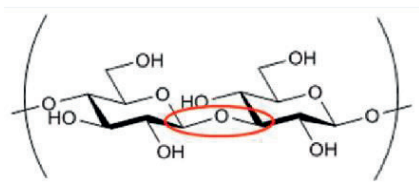

$\beta-1,3$

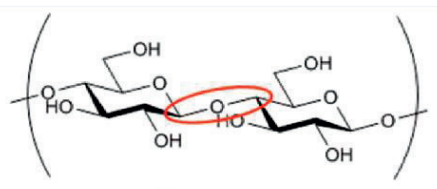

$\beta-1,4$

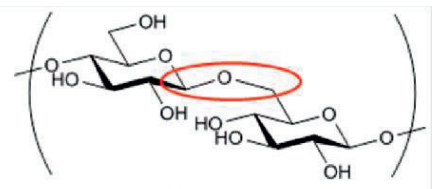

$\beta-1,6$

Figure 1. Structure of $\boldsymbol{\beta}$-glucan molecules. Examples of different linkages between repeating glucose units, determining the biochemical structure of diverse $\beta$-glucans. 


\section{Oral administration of $\beta$-glucans stimulates immune responses in fish}

As mentioned above, $\beta$-glucans can be a potential immuno-stimulant for fish with clear effects, in particular, on innate immunity $(19,21,88,89)$. These effects may not only depend on the branched structure but may also rely on the non-digestible nature of $\beta$-glucans (88). Non-digestible $\beta$-glucans may induce alterations in the composition of the gut microbiota and thereby indirectly influence the immune system (see paragraph 4.1) and/or the bacterial community in the gut may help to digest non-digestible oligosaccharides such as $\beta$-glucans into short-chain fatty acids with a physiological effect of their own (90). Alternatively, the linear $\beta-1,3$ backbone ends up undigested in the proximal part of the intestine, where a proportion is phagocytosed by neutrophilic granulocytes and/or macrophages and degraded by a reactive oxygen species-driven process (91). Of interest, in salmonids, the uptake of laminaran, a linear $\beta-1,3-$ glucan, via the posterior intestine results in a systemic accumulation in, among others, heart and spleen (92) whereas anal intubation with FITC-labelled yeast particles reveals uptake by mononuclear cells in the intestinal lumen (93). The extent to which yeast and $\beta-$ glucan particles are digested and/or taken up is still under debate, but it appears that the teleost intestine certainly is capable of uptake of $\beta$-glucans. The mechanisms behind antigen sampling and the cells involved in this process and present in the teleost gut are reviewed by Løkka and Koppang in this issue. Here, we build on previous reviews of the subject and add a discussion of the more recent literature (2008-2015 in particular) grouped by (super)order, differentiating salmonids, perciforms and cyprinids based on the assumption that the closer the phylogenetic relationship the more reliable the conclusions. While also briefly discussing other routes of administration such as injection and immersion, the more practical route of oral administration through the diet will receive most attention.

\subsection{Salmoniformes (salmonids)}

One of the first studies on the protective effects of $\beta$-glucans, describing that intraperitoneal injection with a $\beta-1,3 / 1,6$ ' $M$ ' glucan from $S$. cerevisiae enhanced resistance against two different bacterial pathogens, was performed in Atlantic salmon (84). It may not come as a surprise that subsequent studies addressing immune-modulating effects of $\beta$-glucans were performed in salmonids in particular. Based on the initial observations that $\beta$-glucans do indeed have immune-stimulating capacities, at least when injected, subsequent studies often included more practical routes such as oral administration (Table 1). The common picture that emerges from the studies on oral administration in salmonids is a confirmation of the immune-stimulating capacity of $\beta$-glucans, although with variable outcomes when it comes to increasing resistance against pathogens. For example, $\beta$-glucan treatment appears to increase resistance of Atlantic salmon to sea lice of the species Lepeophtheirus salmonis but not Caligus elongates (94), for 
which $\beta$-glucan treatment may even lead to a higher infestation (95). Of course, $\beta$-glucans should not be considered miracle compounds able to increase resistance to all pathogens at all levels of infection.

Table 1. Effects of oral administration of $\boldsymbol{\beta}$-glucans in salmonid fish. Publications are grouped according to species, exact type of $\beta$-glucan used as immune stimulant and date of publication. The most pronounced outcomes of each study are summarized as "Results".

\begin{tabular}{|c|c|c|c|}
\hline Species & Stimulant & Results & Reference \\
\hline $\begin{array}{l}\text { Rainbow trout } \\
\text { (Oncorhynchus } \\
\text { mykiss) }\end{array}$ & $\begin{array}{l}\beta-1,3 / 1,6- \\
\text { glucan } \\
\text { (lentinan) }\end{array}$ & $\begin{array}{l}\text { Decreased expression of pro-inflammatory genes in } \\
\text { response to LPS (investigation by micro-array) }\end{array}$ & (96) \\
\hline Rainbow trout & $\begin{array}{l}\text { B-1,3/1,6- } \\
\text { glucan }\end{array}$ & $\begin{array}{l}\text { Increasing trend in lysozyme activity (not significant) } \\
\text { observed in glucan fed trout } \\
\text { Glucan fed trout show increased resistance against } \\
\text { challenge with Ichthyophthirius multifilis (white spot) } \\
\text { Trend visible after short term feeding, significant after } \\
\text { feeding for a longer time period }\end{array}$ & (97) \\
\hline $\begin{array}{l}\text { Atlantic salmon } \\
\text { (Salmo salar) }\end{array}$ & $\begin{array}{l}\beta-1,3 / 1,6- \\
\text { glucan }\end{array}$ & $\begin{array}{l}\text { No significant effect on diarrhoea-like conditions } \\
\text { No implications on soybean meal induced enteritis } \\
\text { No effect on number of sea lice (Caligus elongatus) } \\
\text { infested fish or on number of sea lice per fish } \\
\text { Significant lower salmon lice (Lepeophteirus salmonis) } \\
\text { infestation (infested fish and lice per fish }\end{array}$ & (94) \\
\hline Atlantic salmon & $\begin{array}{l}\beta-1,3 / 1,6- \\
\text { glucan }\end{array}$ & $\begin{array}{l}\text { No effect on sea lice infestation, lice per fish even tends } \\
\text { to be higher than control } \\
\text { Histology showed no adverse effects of glucan enriched } \\
\text { feed on intestines }\end{array}$ & (95) \\
\hline Rainbow trout & $\begin{array}{l}\beta-1,3-\text { glucan } \\
\text { (Euglena } \\
\text { gracilis) } \\
\text { ( } \geq 98 \% \text { purity) }\end{array}$ & $\begin{array}{l}\text { No effect of } \beta \text {-glucan alone or as adjuvant on survival } \\
\text { after Yersinia ruckerii challenge } \\
\text { Down-regulation of expression of pro-inflammatory, } \\
\text { acute phase and lysozyme related genes after } \\
\text { challenge }\end{array}$ & (98) \\
\hline Rainbow trout & $\beta$-1,3-glucan & $\begin{array}{l}\text { Increased gene expression of cathelicidins } 2 \text { and IL-1 } \\
\text { in gut epithelial cells } \\
\text { Increased number of mucus secreting cells in the } \\
\text { intestine }\end{array}$ & (99) \\
\hline
\end{tabular}

Studies investigating the effects of $\beta$-glucans on maintaining the integrity of the gut have found no adverse effects and provide evidence for an assumed favourable increase in frequency of mucus-secreting cells in the epithelial barrier $(95,99)$. Of interest, oral administration of rainbow trout with $\beta$-glucans appears to downregulate the expression of immune-regulatory genes (e.g. IL-1 $\beta$ and lysozyme) in the presence of a microbial stimulus $(96,98)$, but up-regulate the expression of such genes (e.g. IL-1 $\beta$ and cathelicidins (host defense peptides)) in the absence of a microbial stimulus $(98,99)$. These apparent contrasting effects of $\beta$-glucans on the expression of immune-regulatory genes, in the presence or absence of a microbial stimulus, could possibly help explain the variable outcomes with respect to increased resistance against pathogens mentioned above. 
To verify and help explain the initial field observations, the number of laboratorybased studies aiming to acquire more detailed knowledge of the immunestimulating effects of $\beta$-glucans in salmonids, have increased considerably. It hasbecome clear that although the exact recognition receptors and downstream signalling routes still remain undefined, the immune-modulating effects of $\beta$ glucans on the immune system of salmonid fish should be considered stimulatory. Although the degree of disease protection offered by $\beta$-glucans clearly depends on, among others, the infectious agent, it should be noted that oral administration of $\beta$-glucans in salmonid species holds great potential as a prophylactic measure.

\subsection{Perciformes (bass)}

In Nile tilapia, $\beta$-glucans can rescue immune-compromised individuals treated with mercuric chloride, by feeding with a diet containing live $S$. cerevisiae, laminaran or purified $\beta$-glucans (from $S$. cerevisiae) (Table 2 ). The most pronounced effect was observed in fish fed with the purified $\beta$-glucans, where cellular and humoral immune parameters were restored to control levels and protection against subsequent challenge with Aeromonas hydrophila increased from $5 \%$ (control diet) to $60 \%$ survival ( $\beta$-glucan enhanced diet) (100). In most of the recent studies performed on bass species, oral administration of $\beta$-glucans not only increases innate immune parameters, such as phagocytic capacity and oxidative burst, lysozyme and complement activity (100-103), but also increases protection against challenge with a number of bacterial pathogens including $A$. hydrophila and Vibrio alginolyticus $(100,103)$. In general, the observations on the immunestimulating effects of $\beta$-glucans in perciforms (Table 2 ) support the findings in salmonids, with a comparable lack of mechanistic knowledge that could help explain recognition, signalling and immune-stimulation. 
Table 2. Effects of oral administration of $\boldsymbol{\beta}$-glucans in perciform fish. Publications are grouped according to species, exact type of $\beta$-glucan used as immune stimulant and date of publication. The most pronounced outcomes of each study are summarized as "Results".

\begin{tabular}{|c|c|c|c|}
\hline Species & Stimulant & Results & Reference \\
\hline $\begin{array}{l}\text { Nile tilapia } \\
\text { (Oreochromis } \\
\text { niloticus) }\end{array}$ & $\begin{array}{l}\beta-1,3 / 1,6- \\
\text { glucan or } \\
\text { laminaran }\end{array}$ & $\begin{array}{l}\text { Increased phagocytic activity and index in } \\
\text { immunocompromised (IMC) fish due to mercuric } \\
\text { chloride exposure); } \\
\text { Increased oxidative burst and neutrophil adhesion cells } \\
\text { in IMC fish fed } \beta \text {-glucan or laminaran; } \\
\text { Increased survival after challenge with Aeromonas } \\
\text { hydrophila in normal and IMC fish fed } \beta \text {-glucan; }\end{array}$ & $(100)$ \\
\hline $\begin{array}{l}\text { Orange spotted } \\
\text { grouper } \\
\text { (Epinephelus } \\
\text { coioides) }\end{array}$ & $\begin{array}{l}\text { Mixture of } \beta- \\
1,4 ; \beta-1,3 \text { and } \\
\beta-1,6 \text {-glucans }\end{array}$ & $\begin{array}{l}\text { Increased lysozyme activity, alternative complement } \\
\text { activation, phagocytic activity and oxidative burst; } \\
\text { Increased protection against Vibrio alginolyticus } \\
\text { challenge; }\end{array}$ & (103) \\
\hline $\begin{array}{l}\text { Gilthead } \\
\text { seabream } \\
\text { (Sparus aurata) }\end{array}$ & $\begin{array}{l}\beta-1,3 / 1,6- \\
\text { glucan }(99 \% \\
\text { pure) }\end{array}$ & $\begin{array}{l}\text { Increased IL-1 } \beta \text { and IFNy expression; } \\
\text { Increased phagocytosis and phagocytic index; }\end{array}$ & (101) \\
\hline $\begin{array}{l}\text { Red sea bream } \\
\text { (Pagrus major) }\end{array}$ & $\begin{array}{l}\text { Heat killed } \\
\text { Lactobacillus } \\
\text { plantarum } \\
\text { (HKLP) in } \\
\text { combination } \\
\text { with } \beta \text { - } \\
1,3 / 1,6 \text {-glucan }\end{array}$ & $\begin{array}{l}\beta \text {-glucans significant increase the effect of HKLP, with } \\
\text { respect to the lysozyme activity, the bactericidal effect, } \\
\text { the alternative complement pathway activation and the } \\
\text { total serum protein concentrations }\end{array}$ & $(102)$ \\
\hline
\end{tabular}

\subsection{Cypriniformes (cyprinids)}

Increasing attention has been given towards studying immune-modulating effects of $\beta$-glucans in cyprinids. As described above for salmonids and perciforms, also in cyprinids, oral administration of $\beta$-glucans stimulates a suite of innate immune parameters, again including stimulation of phagocytic capacity and oxidative burst, lysozyme and complement activity (104-107) (Table 3). Several studies in cyprinids have addressed the effect of $\beta$-glucan administration on (immune) gene expression (108-111). Among the investigated studies, continuous administration of $\beta$-glucans generally appears to result in an increased expression of proinflammatory genes, with a gradual decline over time depending on, among others, route of administration and immune organ under investigation $(107,108)$. Continuous oral administration (25 days) of $\beta$-glucans can result in the upregulation of anti-apoptotic genes in gut and head kidney, and of both anti- and pro-apoptotic genes in the spleen of common carp (109). The effects of $\beta$-glucans on apoptosis were further investigated and show that, in vitro, $\beta$-glucans can have a significant effect on apoptosis, but only at very high concentrations (112). Taken together, these findings support the notion that oral administration of $\beta$-glucans may modulate the intestinal immune response and protect cyprinid fish from an acute (over)reaction $(108,111)$. Since stimulation requires very high doses of $\beta$ glucans $(500 \mu \mathrm{g} / \mathrm{mL})$ to significantly increase apoptosis in head kidney leukocytes (112), it does not appear that oral administration of $\beta$-glucans has major effects 
on programmed cell death of leukocytes. Strikingly, continuous oral administration of $\beta$-glucans up-regulates the expression of TLR3, a pattern recognition receptor assumed important for the recognition and binding of viral double-stranded RNA, leading to the subsequent triggering of a type-I interferon (IFN) response (111). The link between oral administration of $\beta$-glucans and up-regulation of a receptor for viral pathogen-associated molecular patterns such as dsRNA does not appear an obvious one and requires further investigation, before it can help to explain the mechanism behind protective effects of $\beta$-glucans on resistance against viral pathogens. Overall, it is becoming clear that oral administration of $\beta$-glucans stimulates the innate immune system of cyprinids as it stimulates the innate immune system of salmonid and perciform fish species, suggesting that the capacity to stimulate the innate immune system of fish is a capacity intrinsic to (large molecular weight) $\beta$-glucans.

Table 3. Effects of oral administration of $\beta$-glucans in cyprinid fish. Publications are grouped according to species, exact type of $\beta$-glucan used as immune stimulant and date of publication. The most pronounced outcomes of each study are summarized as "Results".

\begin{tabular}{|c|c|c|c|}
\hline Species & Stimulant & Results & Reference \\
\hline $\begin{array}{l}\text { Common carp } \\
\text { (Cyprinus carpio) }\end{array}$ & $\beta$-1,3-glucan & $\begin{array}{l}\text { Increased oxidative burst } \\
\text { Increased lysozyme activity } \\
\text { Increased protection against Aeromonas hydrophila } \\
\text { challenge }\end{array}$ & (105) \\
\hline $\begin{array}{l}\text { Koi carp } \\
\text { (Cyprinus carpio } \\
\text { koi) }\end{array}$ & $\begin{array}{l}\beta-1,3-\text { glucan, } \\
\text { Chitosan or } \\
\text { Raffinose }\end{array}$ & $\begin{array}{l}\text { Increased white blood cell count (WBC) } \\
\text { Increased oxidative burst, lysozyme activity, } \\
\text { phagocytosis, bactericidal effect }\end{array}$ & (106) \\
\hline Common carp & $\begin{array}{l}\beta-1,3 / 1,6- \\
\text { glucan }\end{array}$ & $\begin{array}{l}\text { Down-regulation of pro-inflammatory genes in gut and } \\
\text { head kidney } \\
\text { Decrease in IgM titer after Aeromonas salmonicida } \\
\text { challenge (i.p injection) } \\
\text { Increased expression of pro-inflammatory genes in } \\
\text { head kidney after challenge but down-regulation in gut }\end{array}$ & $(108)$ \\
\hline Common carp & $\begin{array}{l}\beta-1,3 / 1,6- \\
\text { glucan }\end{array}$ & $\begin{array}{l}\text { No apoptosis in head kidney cells } \\
\text { Up-regulation of several anti- and pro-apoptotic genes } \\
\text { Differential responses between different organs } \\
\text { Upon LPS injection increased expression of pro- } \\
\text { apoptotic genes in head kidney, rest of tested organs } \\
\text { no effect }\end{array}$ & (109) \\
\hline Common carp & $\begin{array}{l}\beta-1,3 / 1,6- \\
\text { glucan }\end{array}$ & $\begin{array}{l}\text { Increased expression of B-Defensin } 1 \text { and } 2 \text { and } \\
\text { Mucin5b in skin and B-Defensin-2 in gills }\end{array}$ & $(110)$ \\
\hline Common carp & $\begin{array}{l}\beta-1,3 / 1,6- \\
\text { glucan }\end{array}$ & $\begin{array}{l}\text { Increased basal CRP levels and alternative complement } \\
\text { activation } \\
\beta \text {-glucan augments the CRP and complement response } \\
\text { to Aeromonas salmonicida challenge } \\
\text { Differential effects observed between organs }\end{array}$ & (107) \\
\hline Common carp & $\begin{array}{l}\beta-1,3 / 1,6- \\
\text { glucan }\end{array}$ & $\begin{array}{l}\text { Reduced expression of immune-regulatory genes in the } \\
\text { midgut (IL-1 } \beta, I L-10 \text { and TNFa) } \\
\text { Mx significantly increased upon poly(I:C) injection }\end{array}$ & (111) \\
\hline Common carp & $\begin{array}{l}\beta-1,3 / 1,6- \\
\text { glucan }\end{array}$ & $\begin{array}{l}\text { Increased serum complement activity } \\
\text { Increased alternative complement activation }\end{array}$ & $(104)$ \\
\hline
\end{tabular}




\section{Injection and immersion routes of administration of $\beta$-glucans}

Although oral administration of $\beta$-glucans clearly is among the most practical applications, other routes of administration have also been investigated (Table 4). Maybe not always cost-effective, but intraperitoneal (i.p.) injection certainly is an effective method to deliver $\beta$-glucans and stimulate the immune system. For example, a single dose of $\beta$-glucans injected i.p. in rainbow trout resulted in a level of protection against infection with the microsporidian, Loma salmonae, similar to the level of protection induced by a 3 weeks feeding trial using 10 times higher concentrations of $\beta$-glucans. Interestingly, the effects of the single i.p. injection could be measured for a prolonged period of up to 9 weeks in vivo (113) and up to 20 days ex vivo (no further time points measured) (114). Protective and stimulating effects on innate immunity after i.p. injection with $\beta$-glucans have also been observed in zebrafish. A single i.p. injection 6 days prior to challenge with $A$. hydrophila reduced the cumulative mortality, with similar although lower effects when injected only 2 days prior to challenge (115). Increasing the frequency of injections further increased the survival of the zebrafish. Although the effects of even a single i.p. injection appear to be rapid and universal, only relatively few studies have addressed this route of administration, while this route could be of wider interest. For example, verification of immune-stimulation after injection of $\beta$-glucans could be of interest for injection vaccination protocols.

A potentially interesting alternative application of immune-stimulation induced by $\beta$-glucans is provided by the immersion treatment of fertilized eggs, or gametes, of chum salmon (Oncorhynchus keta) against infection with Saprolegnia spp.: a short treatment of 3 minutes only was sufficient to provide a significant protection against spontaneous infection with this oomycete (116). This finding seems supported by the observation that both, pro- and anti-inflammatory genes were up-regulated after immersion of rainbow trout fry in a solution containing $\beta$-glucan (117). Comparable to these studies in salmonids, there has been an increasing interest in applying $\beta$-glucans as immersion treatment to cyprinid fish. Although it appears difficult to convincingly show that $\beta$-glucan administration by immersion can (also) have a systemic rather than only a local effect on the immune system (118), $\beta$-glucans can significantly improve wound healing of carp skin when applied to the water (119). Mucosal organs, including the skin, typically are covered by a layer of mucus which is continuously renewed to prevent pathogen attachment and serves as a vehicle for antimicrobial compounds, complement, and immunoglobulins (120). Administration of $\beta$-glucans by immersion, as modulators of mucosal surfaces of the skin or gills could be a promising new area of research, especially now that tools to reliably measure mucosal immunity are becoming available (121). Possible explanations for immune-stimulating effects of $\beta$-glucan immersion baths could be sought, for example, in effects on the composition of microbial communities in the skin mucus (see also paragraph 4.1) (122) or increased local populations of alternatively-activated macrophages expressing a 
healing phenotype (123). Independent of the exact macrophage phenotype that would develop in the presence of $\beta$-glucans, given the most recent indications in humans that trained immunity can be stimulated via recognition of $\beta$-glucans by macrophages (see also paragraph 5 ), there is no doubt that modulation of macrophage function by $\beta$-glucans should remain an active area of research of fish immunology.

Table 4. Effects of routes of $\boldsymbol{\beta}$-glucan administration other than oral. Publications are grouped according to species, route of administration and date of publication. The most pronounced outcomes of each study are summarized as "Results". The bold black border separates studies in Salminiformes from studies in Cypriniformes.

\begin{tabular}{|c|c|c|c|c|}
\hline Species & Stimulant & $\begin{array}{l}\text { Route of } \\
\text { administration }\end{array}$ & Results & Reference \\
\hline $\begin{array}{l}\text { Rainbow trout } \\
\text { (Oncorhynchus } \\
\text { mykiss) }\end{array}$ & $\begin{array}{l}\beta-1,3 / 1,6- \\
\text { glucan (S. } \\
\text { cerevisiae or } \\
\text { ProVale) }\end{array}$ & $\begin{array}{l}\text { Intraperitoneal } \\
\text { (i.p.) injection or } \\
\text { oral administration }\end{array}$ & 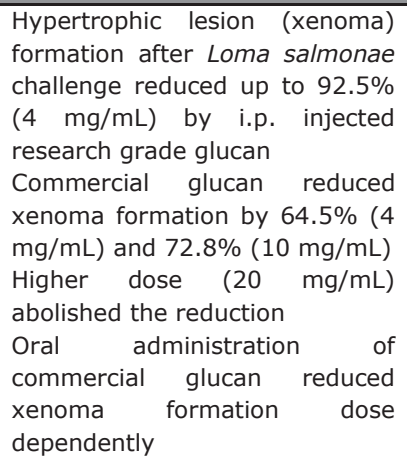 & (113) \\
\hline $\begin{array}{l}\text { Atlantic salmon } \\
\text { (Salmo salar) }\end{array}$ & $\begin{array}{l}\beta-1,3 / 1,6- \\
\text { glucan } \\
\text { (laminaran) }\end{array}$ & $\begin{array}{l}\text { I.p. injection } \\
\text { dissolved in PBS or } \\
\text { encapsulated in } \\
\text { nanoparticles }\end{array}$ & $\begin{array}{l}\text { Two days post injection, } \\
\text { significant up-regulation in TNFa, } \\
\text { IL-1 } 1 \beta \text { and IL-10 expression } \\
\text { Encapsulated } \beta \text {-glucan invoked a } \\
\text { stronger increase in IL-1 } \\
\text { expression than not } \\
\text { encapsulated glucans }\end{array}$ & $(124)$ \\
\hline Atlantic salmon & $\beta$-1,3-glucan & I.p. injection & $\begin{array}{l}\text { Macrophages show significantly } \\
\text { increased oxidative burst } \\
\text { Lysozyme and phagocytic } \\
\text { activity at } 10 \text { and } 20 \text { days post } \\
\text { injection (no further time point } \\
\text { measured) }\end{array}$ & $(114)$ \\
\hline $\begin{array}{l}\text { Chum salmon } \\
\text { (Oncorhynchus } \\
\text { keta) }\end{array}$ & $\begin{array}{l}\beta-1,3 / 1,6- \\
\text { glucans }\end{array}$ & $\begin{array}{l}\text { Treatment of eggs } \\
\text { or gametes with } \\
\text { glucan solution }\end{array}$ & $\begin{array}{l}\text { Increased embryo and juvenile } \\
\text { survival } \\
\text { Increased resistance against } \\
\text { Saprolegnia spp. infection }\end{array}$ & $(116)$ \\
\hline $\begin{array}{l}\text { Zebrafish } \\
\text { (Danio rerio) }\end{array}$ & $\begin{array}{l}\beta-1,3 / 1,6- \\
\text { glucan }\end{array}$ & I.p. injection & $\begin{array}{l}\text { Increased myelomonocytic cell } \\
\text { counts } \\
\text { Increased } \text { pro-inflammatory } \\
\text { cytokine and chemokine } \\
\text { expression } \\
\text { Increased resistance against } \\
\text { Aeromonas hydrophila challenge }\end{array}$ & (115) \\
\hline $\begin{array}{l}\text { Common carp } \\
\text { (Cyprinus carpio) }\end{array}$ & $\begin{array}{l}\beta-1,3 / 1,6- \\
\text { glucan }\end{array}$ & Bath immersion & $\begin{array}{l}\text { Significant increase in wound } \\
\text { healing } \\
\text { Effects on the cytokine } \\
\text { expression profile but differential }\end{array}$ & (119) \\
\hline
\end{tabular}




\section{Mechanisms influencing immune-stimulation by $\beta$-glucans}

Immune-modulatory effects of $\beta$-glucan administration have been widely observed and are generally considered as stimulatory for the health status of fish $(21,88$, $89,122)$. One of the proposed modes of action indicates a prime role for the intestinal immune system, where induction of local intestinal inflammation after administration of $\beta$-glucans would result in a subsequent increased resistance against pathogens (19). A conclusive mode of action explaining the effects of $\beta$ glucans on the immune system of teleosts has yet to be uncovered.

\subsection{Composition of microbial communities}

The immune-stimulating effects of $\beta$-glucans not only depend on their branched structure but also rely on their non-digestible nature (88). Acid-treatment to mimic the effect of stomach-passage, completely abolishes the immune-stimulating effects of $\beta$-glucans on macrophages (125). So far, this disruptive effect of low $\mathrm{pH}$ values has been reported in a single study only, but it could be important to further investigate to which extent (treatments mimicking) digestive processes can abolish the immune-stimulating capacity of $\beta$-glucans when administered orally.

Although beyond the direct scope of this review, one of the modes of action of oral administration of $\beta$-glucans could be to induce alterations in the composition of the gut microbiota. Feeding common carp with $\beta$-glucan-supplemented diets can modulate the microbial communities in the gut (79). Two weeks of feeding appears to reduce diversity, species richness and number of taxonomic units in the autochthonous (mucosal associated, indigenous) microbiota, a reduction not observed after 4 weeks of feeding. However, in another study, two weeks of feeding resulted in a clear increase rather than reduction in microbial community diversity, possibly explained by differences in samples, size of fish and analysis techniques (126). In sea bass fed with $\beta$-glucans for 4 or 8 weeks, pyrosequencing of the intestinal microbiota revealed a transient alteration at the family taxonomic level in the composition of the autochthonous microbiota (127). It took a period of 4 weeks to completely shift the dominance within the microbial communities, which returned to the original composition after another 4 weeks of feeding. The data presented in these studies imply that effects of oral administration of $\beta$-glucans on the microbial composition in the gut are present, but could be transient and require further investigation. In line with these findings; the previously-mentioned effect of long-term feeding with $\beta$-glucans on TLR3 expression in the gut of carp could also be due to an indirect effect of $\beta$-glucans on the composition of the microbiota. In mice, a particular group of commensal bacteria present in the intestine have the ability to induce TLR3 expression, leading to the production of protective IFN$\beta$ (76). Given the immunological importance of the skin, especially in teleost fish (128), it should also be of interest to study changes in composition of the microbial community of the skin after bath treatment with $\beta$-glucans. 


\subsection{Receptor recognition and signalling}

$\beta$-glucans are thought to be internalized by phagocytosis, permitting their destruction by reactive oxygen and nitrogen species and by lytic enzymes in the acidic environment of the phagolysosome, largely based on information in mammals [reviewed by:(86)]. Among the best characterized phagocytic receptors are the opsonising Fc-Y receptor (FcYR) and the complement receptor 3 (CR3). Teleost fish do express genes encoding for the alpha- (CD11(b)-like) and beta-unit (CD18) of CR3 $(129,130)$, but at least zebrafish appear to miss classical members of the FcRs, although the genome does contain ancestral FcR-like genes (131). A distinct class A member of the Scavenger Receptor (SR) family, MARCO (macrophage receptor with collagenous structure), is present in several fish species, and knockdown experiments in zebrafish confirmed a role for MARCO in rapid phagocytosis of Mycobacterium marinum (132). In contrast, a clear role for the class B SR family member, CD36, could not be demonstrated in zebrafish and common carp (133). Toll-like receptors such as TLR2 could possibly sense $\beta$ glucans and are expressed in several fish species (134), but the presence of the prototypical C-type lectin receptor (CLR) for $\beta$-glucan, Dectin-1, is limited to mammalian genomes (135).

In vitro modulation of fish leukocyte function by $\beta$-glucans is an active field of research that might help identify the exact receptor(s) involved and characterize (new) activation routes. For example, recent studies in common carp show that $\beta$ glucans can induce and increase robustness of Neutrophil Extracellular Traps (NETs) $(136,137)$. We studied the production of reactive oxygen and nitrogen species in response to $\beta$-glucans in common carp. Untreated zymosan and branched $1,3 / 1,6 \beta$-glucans induce higher responses than zymosan depleted of TLR-stimulating activity and curdlan, both considered to be Dectin-1-specific ligands (138). The latter finding suggests recognition of $\beta$-glucans by multiple pattern recognition receptors on carp macrophages. Clearly, the relative importance of phagocytic receptors such as CR3, members of the SR and CLR superfamilies, and sensing receptors such as TLR2 with respect to $\beta$-glucanstimulated immune responses has yet to be defined for fish. Identification of a Ctype lectin receptor in fish with a role equivalent to the Dectin-1 receptor would be a crucial first step to subsequent studies of function based on, for example, the production of soluble receptors for $\beta$-glucan binding and carbohydrate competition assays.

\subsection{Trained immunity}

As mentioned earlier (paragraph 1), recent insights in the field of innate immunity provide indications that $\beta$-glucans could have effects over a longer timespan than initially anticipated, that might be explained by the phenomenon trained immunity. Traditionally, innate immune responses are characterized as rapid (hours-days), aspecific and without development of specific memory. In contrast, acquired immune responses are characterized as slow (days to weeks), highly specific 
(variable receptors on $\mathrm{T}$ and $\mathrm{B}$ lymphocytes can specifically recognize almost any antigen) and expressing long-term memory (based upon clonal expansion of memory $\mathrm{T}$ and/or $\mathrm{B}$ cells). Already, the classification of innate immune responses as aspecific has been challenged and thus, based on the presence of numerous classes of pattern recognition receptors $(25,139)$ innate immunity no longer is classified as aspecific. At present, also the strict absence of a form of memory for innate immune responses is challenged by a new concept named trained immunity (Figure 2), which is characterized by three criteria: (i) it can be induced after a primary infection or immunization and subsequently provide protection against a secondary infection in a T- and B-lymphocyte independent manner, (ii) it may be less specific than the adaptive immune response but still confers increased resistance upon reinfection of the host and, (iii) innate cell types such as macrophages and natural killer cells are key players in the mechanism, which involves improved pathogen recognition and an increased inflammatory response (56).

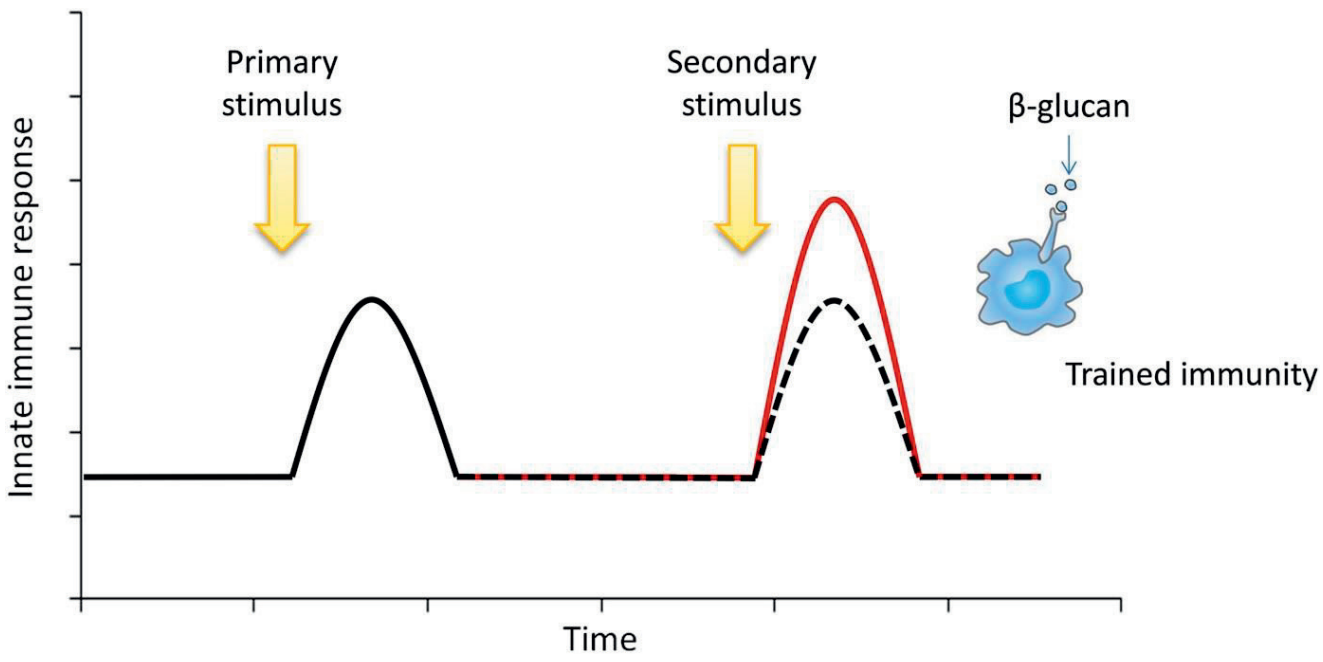

Figure 2. The concept of trained immunity. Classical view on repeated stimulation of innate immunity (dotted black line) and updated view including trained immune responses (solid red line), [adapted from: $(57,66)]$. Arrows depict the moment of first and second stimulation with, for example, $\beta$-glucans. In the classical view, primary and secondary responses are of equal magnitude, whereas trained immunity results in a heightened secondary response facilitated by cells of the innate immune system.

Of particular interest with respect to the present review, are observations in humans and mice on long-lived immune-modulatory effects of purified $\beta$-glucans and/or $C$. albicans on the development of trained immunity. In humans, preincubation of peripheral blood monocytes (PBMCs) in vitro with a single dose of $C$. albicans or $\beta$-glucans thereof, results in an increased cytokine production of, among others, IL-6 and TNFa, upon secondary stimulation with the same glucans for a relatively long period of up to 2 weeks (58). Besides the increase in cytokine production, a metabolic shift is also observed; pre-incubated PBMCs have 
significantly higher glucose consumption and lactate production than control cells (62). In mice, pre-treatment with highly purified $\beta$-glucans in vivo, protects animals from a subsequent infection with an unrelated pathogen, such as Staphylococcus aureus (140). The innate nature of this form of cross protection was investigated in recombination-activating gene (Rag) knock-out (KO) mice which lack functional B- and T-cells and thus lack functional adaptive immunity. The Rag-KO mice could be fully protected from a lethal infection with C. albicans, but only when pre-stimulated with a sub-lethal dose of C. albicans (58), a phenomenon proposed to be monocyte-dependent. Despite clear in vitro effects, oral administration of $\beta$-glucans appears not to induce pronounced long-lived effects in vivo, in humans (141). However, administration of Bacillus CalmetteGuérin (BCG) does clearly induce trained immunity also in vivo. Effects induced by vaccination with $\mathrm{BCG}(65,142)$, prepared from attenuated live Mycobacterium bovis, support the proposed benchmarks of trained immunity that it can elicit cross-specific protection in a $\mathrm{T}$ - and $\mathrm{B}$-cell independent manner with innate immune cell types such as macrophages acting as key players (56). Of evolutionary interest, long before the recent discussions on the presence of trained immunity in humans and mice (67), similar cross-specific protection was observed in plants (143-145) and invertebrates (68) which, typically without $T$ and $B$ lymphocytes, can build up a form of immunity able to protect the organism upon a secondary exposure. Owing to the basal position of teleost fish as early vertebrates, it makes evolutionary sense to expect that trained immunity could be an important mechanism determining immune-stimulation of fish by $\beta$-glucans.

\section{Future perspectives}

\subsection{Evidence for the presence of trained immunity in fish}

Although teleost fish are among the evolutionarily oldest vertebrates with both an innate and classical adaptive immune system (146), there are several examples where innate immune parameters are more active and more diverse in fish than in mammals (147). In line with these findings one would expect trained immunity should not only be present, but could have a pronounced role in the immune system of fish. There are at least a few studies providing preliminary evidence for the presence of a form of trained immunity in fish, primarily based on experiments with mycobacteria. Already in 1986, Olivier et al. observed a long-lived increase in phagocytic activity of peritoneal macrophages from Brook trout (Salvelinus fontinalis), for a period up to 33 days after i.p. injection with Modified Freund's Complete Adjuvant (MFCA) containing killed Mycobacterium butyricum. Only macrophages from trout injected with MFCA showed a significantly higher bactericidal activity (148). Almost three decades later, the efficacy of injection with (modified) Mycobacteria was further investigated using BCG. Vaccination of Japanese flounder (Paralichthys olivaceus) with BCG resulted in an up-regulation of pro-inflammatory cytokines and conferred protection against Mycobacterium $s p$. (149). Also vaccination of Amberjack (Seriola dumerili) with BCG led to protection 
against challenge with Mycobacterium sp. (150). Importantly, these researchers could measure cross-specific protection, one of the proposed benchmarks of trained immunity. The cross-specific protection could be induced in Japanese flounder by BCG, shown by challenge with Nocardia seriolae, and was possibly mediated by bacteriolytic activity of the serum (151). The other benchmark of trained immunity; that cross-specific protection occurs in a T- and B-cell independent manner (56), was also studied in fish. Exposure of Rag-KO zebrafish to a sub-lethal infection with Edwardsiella ictaluri significantly protected the same animals from a subsequent lethal infection with the same bacteria. Crucially, protection could be transferred to naïve Rag-KO individuals by injection with kidney leukocytes from animals pre-exposed to the sub-lethal infection (152). Together, these studies provide first indications for our hypothesis that trained immunity should be present in teleost fish and provide arguments that the innate immune system of fish can be trained by pre-exposure to sub-lethal pathogens. It remains to be investigated if trained immunity indeed has the predicted, pronounced role in the immune defense of fish and is indeed mediated by innate immune cell types such as macrophages.

\subsection{Trained immunity induced by $\beta$-glucans: revisiting the literature}

In mammalian monocytes, $\beta-1,3$-glucans purified from $C$. albicans are potent inducers of trained immunity $(58,62,140)$. Until this moment, no studies have (knowingly) investigated induction of trained immunity in teleost fish in after administering $\beta$-glucans. After a first screen of the literature for clear indications of $\beta$-glucan-induced trained immunity in fish, we realized it was difficult to unambiguously ascribe observed effects to at least one of the characteristic criteria defined for trained immunity [paragraph 5.3 and (56)]. Therefore, based on the assumption that innate immune responses generally are short-lived, we rescreened the literature for indications of long-lived effects on innate immunity after administering $\beta$-glucans (Table 5).

For example, a single i.p. injection of zebrafish (1-1.5 g body weight) with $\beta$ $1,3 / 1,6$-glucan provided partial protection against challenge with $A$. hydrophila at 6 days, but not at 2 or 4 days post-injection (115). Similarly, but spanning a much longer time period, i.p. injection of yellowtail (Seriola quinqueradiata) with $\beta$ 1,3/1,6-glucan induced increased resistance upon challenge with Pasteurella piscicida for a prolonged period of up to 45 days post-injection (153). Also, i.p. injection of Blue Gourami (Trichopodus trichopterus) with $\beta-1,3-D$-glucan (laminarin), induced increased resistance upon infection with $A$. hydrophila for a prolonged period of 22 days post-injection, which was the last time point measured. Of interest, the latter study provided insights into possible protective mechanisms by measuring an increase in phagocytosis-induced oxidative burst, suggestive of a key role for innate immune cell types such as macrophages (154). Indeed, phagocytes could be responsible for long-lived effects induced by $\beta$ glucans since i.p. injection of Atlantic salmon with $\beta-1,3-$ glucan leads to an increase in oxidative burst, phagocytosis and lysozyme activity of macrophages 
(114). Most interesting in the present context; the latter study showed that increased macrophage activity was still measurable at 10-20 days post-injection, providing clear indications that single i.p. injections with $\beta$-glucans can induce long-lived effects in fish.

Table 5. Innate immune memory-related effects of $\beta$-glucan administration in fish. Publications are presented according to order of discussion in the text. The column "Experimental set up" gives a concise summary of the parameters such as timing and route of administration. The most pronounced outcomes of each study are summarized as "results".

\begin{tabular}{|c|c|c|c|c|}
\hline Species & Stimulant & Experimental set up & Effects & Reference \\
\hline $\begin{array}{l}\text { Zebrafish } \\
\text { (Danio rerio) }\end{array}$ & $\begin{array}{l}\beta-1,3 / 1,6- \\
\text { glucan }\end{array}$ & $\begin{array}{l}\text { Intraperitoneal injection } 6,4 \\
\text { or } 2 \text { days prior to bacterial } \\
\text { challenge. }\end{array}$ & $\begin{array}{l}\text { Lowest cumulative } \\
\text { mortality upon injection } \\
6 \text { days prior to the } \\
\text { bacterial challenge }\end{array}$ & $(115)$ \\
\hline $\begin{array}{l}\text { Yellowtail } \\
\text { (Seriola } \\
\text { quinqueradiata) }\end{array}$ & $\begin{array}{l}\beta-1,3 / 1,6- \\
\text { glucan }\end{array}$ & $\begin{array}{l}\text { Intraperitoneal injection on } \\
\text { day } 0 \text { followed by a bacterial } \\
\text { challenge on } 15,25,35 \text { or } 45 \\
\text { days post injection }\end{array}$ & $\begin{array}{l}\text { Minor increase in RPS } \\
\text { (relative percentage } \\
\text { survival) upon challenge } \\
\text { on day } 15 \text { and } 45 \text { post } \\
\text { injection }\end{array}$ & $(153)$ \\
\hline $\begin{array}{l}\text { Blue gourami } \\
\text { (Trichopodus } \\
\text { trichopterus) }\end{array}$ & $\begin{array}{l}\beta-1,3-D- \\
\text { glucan } \\
\text { (Laminarin) }\end{array}$ & $\begin{array}{l}\text { Intraperitoneal injection } \\
\text { followed by bacterial } \\
\text { challenge } 14 \text { or } 22 \text { days post } \\
\text { injection, or by chemilumine- } \\
\text { scence assay } 8,14 \text { and } 22 \\
\text { days post injection }\end{array}$ & $\begin{array}{l}\text { Increased oxidative } \\
\text { burst in phagocytes up } \\
\text { to } 22 \text { days post injection } \\
\text { Increased survival upon } \\
\text { challenge } 22 \text { days post } \\
\text { injection }\end{array}$ & $(154)$ \\
\hline $\begin{array}{l}\text { Atlantic salmon } \\
\text { (Salmo salar) }\end{array}$ & $\beta$-1,3-glucan & $\begin{array}{l}\text { I.p. injection of } \beta \text {-glucan } \\
\text { solution. Isolation of } \\
\text { macrophages } 10 \text { and } 20 \text { days } \\
\text { post injection. }\end{array}$ & $\begin{array}{lr}\begin{array}{l}\text { Significant } \\
\text { oxidative }\end{array} \\
\text { lysozyme } & \text { burst, } \\
\text { phagocytic activity at } 10 \\
\text { and } 20 \text { days post } \\
\text { injection }\end{array}$ & $(114)$ \\
\hline $\begin{array}{l}\text { Rainbow Trout } \\
\text { (Oncorhynchus } \\
\text { mykiss) }\end{array}$ & $\begin{array}{l}\beta-1,3 / 1,6- \\
\text { glucan }\end{array}$ & $\begin{array}{l}\text { Oral administration of } \\
\text { glucans. Subjects were fed } \\
\text { control diet for } 4 \text { weeks, } \\
\text { experimental diet for } 2 \text { weeks } \\
\text { and finally } 4 \text { weeks of control } \\
\text { diet. }\end{array}$ & $\begin{array}{l}\text { Glucans increase ConA } \\
\text { induced proliferation of } \\
\text { PBLs Glucans increase } \\
\mathrm{Ab} \text { titer in units/uL } \\
\text { Increase in complement } \\
\text { activation } 8 \text { weeks post } \\
\text { vaccination (vaccination } \\
\text { on week } 0 \text { ) }\end{array}$ & $(155)$ \\
\hline $\begin{array}{l}\text { Sea Bass } \\
\text { (Dicentrarchus } \\
\text { labrax) }\end{array}$ & $\begin{array}{l}\beta-1,3 / 1,6- \\
\text { glucan }\end{array}$ & $\begin{array}{l}\text { Oral } \quad \text { administration } \quad \text { of } \\
\text { glucans. } \quad \text { Subjects were } \\
\text { sampled } 4 \text { weeks after the } \\
\text { last feeding cycle }\end{array}$ & $\begin{array}{lr}\begin{array}{l}\text { Increased } \\
\text { complement }\end{array} & \text { activity } \\
\text { and lysozyme activity }\end{array}$ & $(156)$ \\
\hline $\begin{array}{l}\text { Orange spotted } \\
\text { grouper } \\
\text { (Epinephelus } \\
\text { coioides) }\end{array}$ & $\begin{array}{l}\text { Mixture of } \beta- \\
1,4 ; \beta-1,3 \text { and } \\
\beta-1,6-\text { glucans }\end{array}$ & $\begin{array}{l}\text { Oral administration for } 12 \\
\text { days followed by bacterial } \\
\text { challenge at } 0,3,6,9,12 \text { and } \\
15 \text { days after switching back } \\
\text { to control diet. }\end{array}$ & $\begin{array}{l}\text { Increased survival from } \\
\text { bacterial challenge (up } \\
\text { to } 15 \text { days after } \\
\text { switching to control } \\
\text { diet) }\end{array}$ & (103) \\
\hline
\end{tabular}


Continuous feeding with $\beta$-glucans for a number of subsequent days also appears to induce long-lived effects on the immune system of fish. For example, rainbow trout fed with $\beta-1,3 / 1,6$-glucans for a period of 2 weeks still showed higher antibody responses after vaccination against enteric redmouth disease and higher concanavalin A-induced proliferation of head kidney derived leukocytes 4 weeks after switching back to a control diet (155). Sea bass fed with $\beta-1,3 / 1,6$-glucanenriched diets for 3 feeding cycles of 2 weeks followed by 10 weeks of control diet, showed higher serum complement and lysozyme activity than fish fed a control diet only (156). Grouper fed a diet containing a mixture of mushroom-derived $\beta$ 1,$4 ; \beta-1,3$ and $\beta-1,6$-glucans for a continuous period of 12 days still showed higher protection against challenge with $V$. alginolyticus 15 days after switching back to a control diet (103).

\section{Concluding remarks}

The studies discussed here provide indications in existing literature for long-lived effects stimulated by $\beta$-glucans, possibly based on key roles for macrophages. There also are several studies that support the notion that the innate immune system of teleost fish can be trained. Whether $\beta$-glucan administration, either by injection, bath or oral route, indeed triggers trained immunity in a manner similar to what is observed for humans and mice requires more detailed studies specifically designed to investigate the phenomenon of trained immunity in fish. For example, it could be of interest to analyse, in detail, long-lived effects of stimulation with $\beta$-glucans in in vitro models based on purified populations of innate immune cell types, among which macrophages. Such studies would provide fundamental knowledge on mechanisms basal to trained immunity and conserved in cold- and warm-blooded vertebrates.

\section{Acknowledgements}

Annelieke Wentzel from the Cell Biology and Immunology group is gratefully acknowledged for her comments on the manuscript. Research leading to this review was funded by the Netherlands Organisation for Scientific Research (NWO) and São Paulo Research Foundation, Brazil (FAPESP) as part of the Joint Research Projects BioBased Economy NWO-FAPESP Programme (Project number 729.004.002). 



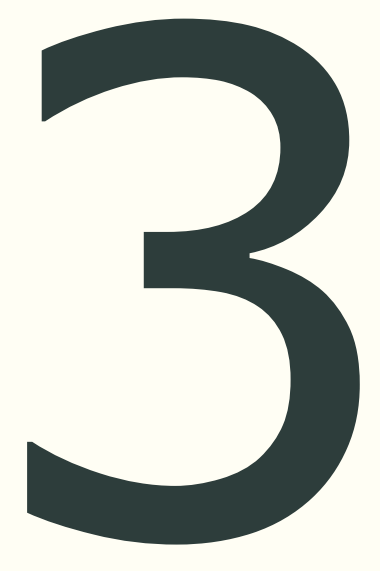

\section{Evidence of trained immunity in teleost fish: conserved features in carp macrophages}

Jules Petit, Carmen W.E. Embregts, Maria Forlenza, Geert F. Wiegertjes

The Journal of Immunology, accepted for publication

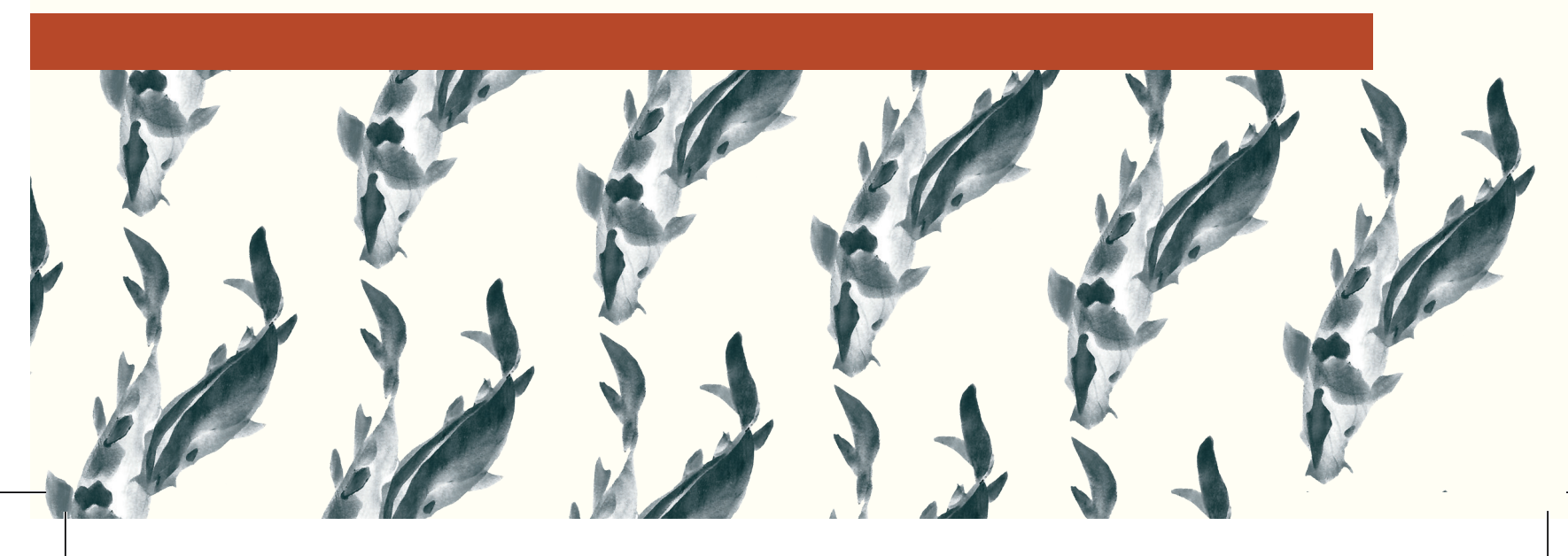




\section{Abstract}

Trained immunity is a form of innate immune memory best described in mice and humans. Clear evidence of the evolutionary conservation of trained immunity in teleost fish is lacking. Given the evolutionary position of teleosts as early vertebrates with a fully developed immune system, we hypothesize that teleost myeloid cells show features of trained immunity common to those observed in mammalian macrophages. These would at least include the ability of fish macrophages to mount heightened responses to a secondary stimulus in a non-specific manner. We established an in vitro model to study trained immunity in fish by adapting a well-described culture system of head kidney-derived macrophages of common carp. A soluble NOD-specific ligand and a soluble $\beta$-glucan were used to train carp macrophages, after which cells were rested for six days prior to exposure to a secondary stimulus. Unstimulated trained macrophages displayed evidence of metabolic reprogramming, as well as heightened phagocytosis and increased expression of the inflammatory cytokines il6 and tnfa. Stimulated, trained macrophages showed heightened production of reactive oxygen and nitrogen species as compared to the corresponding stimulated but untrained cells. Measurement of the production of reactive oxygen species proved particularly informative to identify ligands able to train carp macrophages. We discuss the value of our findings for future studies on trained immunity in teleost fish. 


\section{Introduction}

Traditionally, innate immune responses have been viewed as rapid, relatively nonspecific and lacking immunological memory. New insights have challenged this view, introducing a novel concept referred to as trained immunity, which is defined as a heightened response to a secondary infection that can be exerted toward both homologous and heterologous microorganisms (56). Typical criteria of trained immunity include: 1) induction upon primary infections or immunizations and subsequent protection against a secondary infection, in a T- and B-lymphocyte independent manner, 2) a response that is less specific than an adaptive immune response but that still confers increased resistance upon reinfection of the host and, 3) the involvement of innate cell types such as NK cells and macrophages involved in improved pathogen recognition and an increased inflammatory response.

Initial experiments showed that $\mathrm{Rag}^{-/-}$mice exposed to a sub-lethal Candida albicans infection were better protected against a subsequent lethal dose of these yeasts, whereas monocyte-deficient mice did not show this increased protection, suggesting a form of memory present in the myeloid compartment. Furthermore, in vitro re-exposure of human PBMC to $C$. albicans induced a significant IL6 and TNFa response, even after a resting period of six days following primary exposure to C. albicans. Activation of the Dectin-1/RAF proto-oncogene serine/threonineprotein kinase (Raf-1) pathway in PBMCs by $\beta$-glucans present in the cell wall of C. albicans proved crucial for inducing trained immunity (58). Concurrently, nonspecific protection induced by Bacillus Calmette-Guérin (BCG) vaccination also resulted in increased cytokine production in monocytes upon secondary exposure to unrelated pathogens in a T- and B-cell independent manner. This process, active for prolonged periods of up to three months after vaccination, proved dependent on recognition by nucleotide-binding oligomerization domain-containing protein 2 (NOD2) and signalling via receptor-interacting serine/threonine-protein kinase 2 (Rip2) (65). These studies highlighted that the pathways linked to Dectin-1/Raf-1 ( $\beta$-glucans) and/or NOD2/Rip2 (BCG) could be considered central to stimulation of trained immunity in monocytes.

Further studies that sought to unravel the underlying mechanisms of trained immunity revealed crucial roles for epigenetic modifications and metabolic reprogramming. Trained immunity, induced by exposure of monocytes to $\beta$ glucans or BCG, was associated with long-lived epigenetic modifications in the form of increased trimethylation of histone $3(\mathrm{H} 3)$ lysine $4(\mathrm{H} 3 \mathrm{~K} 4 \mathrm{me} 3)$ and acetylation of $\mathrm{H} 3$ lysine 27 (H3K27ac), both activation markers (58,62, 64), and with decreased trimethylation of $\mathrm{H} 3$ lysine 9 (H3K9me3), a repressor marker (60). These epigenetic modifications, positioned at promotor sites of immune genes, helped explain the heightened IL6 and TNFa response in PBMC after a resting phase of six days following primary exposure, and appear key to the inflammatory response associated with trained immunity. Epigenetic modifications were also noted at promotor sites of metabolic genes, among which the mechanistic target 
of rapamycin kinase (mTOR) and hexokinase 2 (HK2), introducing metabolic reprogramming as a mechanism underlying trained immunity. Indeed, crucial to the onset of trained immunity appears to be a metabolic shift from oxidative phosphorylation towards glycolysis, orchestrated via the protein kinase $B$ (Akt)/mTOR/hypoxia-inducible factor $1 \alpha$ ( $\operatorname{HIF} 1 \alpha)$ pathway $(62,64)$. Transcriptome analysis in mice, comparing constitutive expression of trained and untrained monocytes, revealed significantly higher expression of several genes associated with glycolysis, among others aldehyde dehydrogenase (Aldh2), ADP-dependent glucokinase (Adpgk) and bisphosphoglycerate mutase (Bpgm) (62). Further, activation of the cholesterol synthesis pathway was also noted as an important mechanism underlying trained immunity (61). The metabolic shift resulted in the accumulation of several metabolites such as fumarate, a substrate of the TCA cycle and mevalonate, an intermediate in the cholesterol synthesis pathway. Both metabolites could, by themselves, induce epigenetic modifications and induce cytokine profiles associated with trained immunity (59-61). In addition, blocking metabolic shifts towards glycolysis or glutaminolysis abolished these epigenetic modifications and cytokine profiles, illustrating the strong connections between epigenetic modifications, metabolic reprogramming and trained immunity.

Little or nothing is known about the conservation of aspects of trained immunity in fish, including the aspects described above. Two recent studies in zebrafish have touched upon such aspects, including observations on an increased antiviral state in $\mathrm{rag}^{-/-}$zebrafish associated with increased transcription of innate immune genes (157), and a study describing (the lack of) effects of pre-exposure to different pathogen associated molecular patterns on subsequent viral challenges (158). Given the evolutionary position of teleost fish as early vertebrates with a fully developed immune system (146), it is likely that innate immune cells of fish such as macrophages should possess the ability to express specific features of trained immunity. In fact, given that numerous examples exist of a relatively prominent role of innate immunity in fish $(147,159-162)$, trained immunity could be considered highly relevant to this group of cold-blooded vertebrates. Recently, a review summarized potential benefits and constraints of exploiting trained immunity to our benefit in larval aquaculture (163). We already summarized longlived effects of $\beta$-glucans in fish (20) and hypothesized these might well be explained by features of trained immunity.

In this study, we sought to investigate the hypothesis that trained immunity is conserved in the teleost fish, common carp. To this end, a well-established in vitro culture of macrophages derived from the head kidney (164), the fish equivalent to mammalian bone marrow, was adapted to study the mechanisms of trained immunity in common carp. Given that the existence of a true Dectin-1/Raf-1-like pathway in fish is still elusive (42), but that a NOD2/Rip2-like pathway was proven to exist in zebrafish (165-167), we used a soluble NOD-specific ligand to train carp macrophages. Following a resting period of six days, alike the experimental setup used to study trained immunity in human PBMC (168), unstimulated trained macrophages showed increased phagocytosis, elevated constitutive expression of 
several immune- and glycolysis-related genes as well as a metabolic shift from oxidative phosphorylation towards glycolysis, the latter measured as increased production of lactate. Stimulated trained macrophages typically showed an increased inflammatory response measured as increased production of reactive oxygen species (ROS) and nitrogen radicals (NO). Altogether, our data provide evidence that innate immune cells of teleost fish, such as macrophages, possess the ability to express specific features of trained immunity.

\section{Methods}

\section{Animals}

European common carp (Cyprinus carpio carpio L.) of the R3 $\times$ R8 strain were used, which results from crossing the Hungarian R8 strain and the Polish R3 strain (169). Carp were bred and raised in the aquatic research facility (Carus-ARF) of Wageningen University, at $20-23^{\circ} \mathrm{C}$ in recirculating UV-treated water and fed pelleted dry food (Skretting, Nutreco) twice daily. All experiments were performed with the approval of the animal experiment committee of Wageningen University (DEC number: 2017.W-0034).

\section{Macrophage culture and training stimulus}

Carp were euthanized with $0.3 \mathrm{~g} / \mathrm{L}$ Tricaine Methane Sulfonate (TMS) (Crescent Research Chemicals, Phoenix, USA) in aquarium water buffered with $0.6 \mathrm{~g} / \mathrm{L}$ sodium bicarbonate and bled via the caudal vein. Head kidney was isolated and total head kidney leukocytes were separated on a Percoll ${ }^{\mathrm{TM}}$ (GE healthcare, Fisher Scientific) density gradient. Macrophages were obtained by culturing head kidney leukocytes in complete NMGFL15 medium at $17.5 \times 10^{6}$ cells/flask $\left(75-\mathrm{cm}^{2}\right.$, Corning (CORN430725U)) for 6 days at $27^{\circ} \mathrm{C}$ without $\mathrm{CO}_{2}$ as previously described (164). From here onwards 6-days cultured, head kidney-derived macrophages will be referred to as 'macrophages'.

Culture flasks were placed on ice for $15 \mathrm{~min}$, macrophages were harvested by gentle scraping, washed once with ice-cold PBS and centrifuged at $450 \times g$ for 10 min. Subsequently, cells were resuspended in RPMI-1640 culture medium with 25 mM Hepes, supplemented with L-glutamine $(2 \mathrm{mM})$, penicillin $\mathrm{G}(100 \mathrm{U} / \mathrm{ml})$, streptomycin sulphate $(50 \mathrm{mg} / \mathrm{ml})($ 'CRPMI') and heat-inactivated pooled carp serum $(2.5 \% \mathrm{v} / \mathrm{v})$ ('CRPMI-2.5').

For selection of the optimal concentration of training stimulus, production of reactive oxygen species (ROS) and of nitric oxide (NO) was measured according to the protocol described below. For NO production, macrophages were seeded at a density of $5 \times 10^{5}$ cells/well in 96-well culture plates (Corning, CORN3596) and stimulated for $24 \mathrm{~h}$ with various concentrations of the NOD-ligand 'soluble sonicated peptidoglycan from E. coli K12' (PGN-ECndss, Invitrogen) resuspended in endotoxin-free LAL water, from now on referred to as 'PGN'. For ROS production, 
macrophages were seeded at a density of $5 \times 10^{5}$ cells/well in white 96 -well plates (Corning, CLS3912) and stimulated for $2 \mathrm{~h}$ with various concentrations of PGN.

\section{Optimization of the training period}

Macrophages were obtained as described above, and the training stimulus $(1 \mu \mathrm{g} / \mathrm{ml}$ PGN) was added directly to the culture flask ( $20 \mu \mathrm{L}$ of a $1 \mathrm{mg} / \mathrm{mL}$ solution in 20 $\mathrm{mL}$ medium) for $2 \mathrm{~h}$ at $27^{\circ} \mathrm{C}$ without $\mathrm{CO}_{2}$. Cells were harvested as described above and washed $3 x$ in ice-cold PBS containing $0.02 \%(w / v)$ EDTA (PBS-EDTA) to remove any unbound stimulus. Cells were then seeded at a density of $5 \times 10^{5}$ cells/well in 96-well culture plates and either incubated in cRPMI supplemented with heat-inactivated pooled carp serum $(1.5 \% \mathrm{v} / \mathrm{v})$ (CRPMI-1.5) or stimulated for additional $24 \mathrm{~h}$ with $1 \mathrm{\mu g} / \mathrm{ml}$ PGN. NO production in the cell culture supernatants was measured as described below.

\section{In vitro set-up for studying trained immunity}

Macrophages were trained by adding PGN directly to the flask at a final concentration of $1 \mu \mathrm{g} / \mathrm{mL}$ and incubated for $2 \mathrm{~h}$ at $27^{\circ} \mathrm{C}$ in the absence of $\mathrm{CO}_{2}$. Alternatively, laminarin (tIrl-lam, InvivoGen) at a concentration of $50 \mu \mathrm{g} / \mathrm{mL}$ was added $(100 \mu \mathrm{L}$ of a $10 \mathrm{mg} / \mathrm{mL}$ solution in LAL water, in $20 \mathrm{~mL}$ medium). As a control, cells were exposed to LAL water in a volume equal to the training stimulus. Cells were then harvested as described above and washed $3 x$ in ice-cold PBS-EDTA to remove any unbound stimulus. The obtained cells will be referred to as 'trained macrophages' or 'untrained macrophages'. Sub-sets of trained and untrained macrophages were always analysed for cell viability with Trypan blue exclusion, and for reactivity with an NO assay, as described in section 2.6. The remainder of the cells was seeded at $2.5 \times 10^{6}$ macrophages in $20 \mathrm{~mL}$ of cRPMI-2.5 in $75-\mathrm{cm}^{2}$ culture flasks (T-75 TC treated, 0030711114, Eppendorf). Cells were incubated at $27^{\circ} \mathrm{C}$ for additional six days (resting period) before harvesting as described above. Subsequently, trained and untrained macrophages were washed once with ice-cold PBS, centrifuged at $450 \times g$ for $10 \mathrm{~min}$ and resuspended in cRPMI. Cell growth, viability and morphology of trained macrophages after the resting period were comparable to that of untrained macrophages. A schematic overview of the in vitro set-up is depicted in Figure 1.

\section{Reactive oxygen species production}

Production of reactive oxygen species (ROS) was determined by a real time luminol-ECL assay, as previously described (170). Trained or untrained macrophages were seeded at a density of $5 \times 10^{5}$ cells/well in white 96 -well plates (Corning, CLS3912) and stimulated with: zymosan (tlrl-zyd, InvivoGen, 250 $\mu \mathrm{g} / \mathrm{mL}$; heterologous stimulus), PGN (10 $\mathrm{g} / \mathrm{mL}$; homologous stimulus), cRPMI (unstimulated control), PMA (P8139, Sigma-Aldrich, $1 \mu \mathrm{g} / \mathrm{mL}$; stimulated control). Chemiluminescence emission was measured in real time (every 2 min for $120 \mathrm{~min}$ ) 


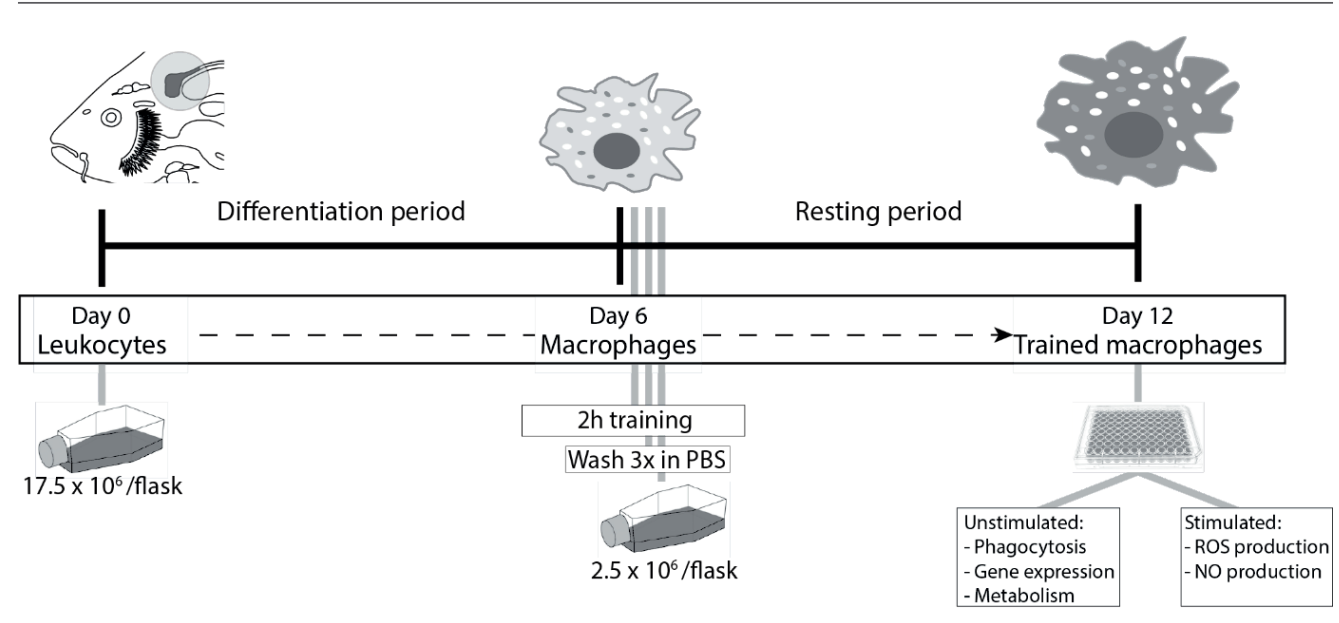

Figure 1. schematic representation of the in vitro experimental set up to obtain trained macrophages. On day 0 , leukocytes are collected from common carp head kidney and cultured for 6 days to allow for differentiation into macrophages (differentiation period). On day 6, macrophages are or are not exposed to the training stimulus for $2 \mathrm{~h}$ in the culture flask. Subsequently, cells are harvested and washed 3 times in ice-cold PBSEDTA. Cells are transferred to new culture flasks at a fixed density per flask and cultured for another 6 days (resting period). On day 12, trained macrophages are harvested and used for subsequent analysis. Macrophages not exposed at day 6 were treated similarly and served as untrained controls.

with a FilterMax F5 Multi-Mode Microplate Reader at $27^{\circ} \mathrm{C}$ and expressed as area under the curve. Fold changes were calculated as the area under the curve of trained or untrained stimulated macrophages relative to untrained unstimulated control (cRPMI).

\section{Nitrogen radicals production}

Production of nitrogen radicals (NO) was determined as nitrite accumulation using the Griess reaction, as previously described (171). Trained or untrained macrophages were seeded at a density of $5 \times 10^{5}$ cells/well in 96 -well culture plates (Corning, CORN3596) and stimulated with: zymosan $(250 \mu \mathrm{g} / \mathrm{mL}$; heterologous stimulus), PGN (10 $\mathrm{gg} / \mathrm{mL}$; homologous stimulus), cRPMI (unstimulated control), LPS (L2880, Sigma-Aldrich, $50 \mu \mathrm{g} / \mathrm{mL}$; stimulated control). After $15 \mathrm{~h}$ at $27^{\circ} \mathrm{C}$ in the presence of $5 \% \mathrm{CO}_{2}$, nitrite production was measured at $\mathrm{OD}_{540}$, using a FilterMax F5 Multi-Mode Microplate Reader and quantified using a sodium nitrite $\left(\mathrm{NaNO}_{2}\right)$ standard curve. Fold changes were calculated as production of nitrite by trained or untrained stimulated macrophages relative to untrained unstimulated control (CRPMI).

\section{Phagocytosis analysis}

Analysis of phagocytic capacity was performed by flow cytometry (FACS) as previously described (172), with minor modifications. Briefly, trained or untrained macrophages ( $5 \times 10^{4}$ cells/well in 96-well cell culture plates (Corning, CORN3596)) were incubated with fluorescent beads (PSF-001UM Red, MagSphere; cell:bead 
ratio of $1: 10$ ) for $120 \mathrm{~min}$ at $27^{\circ} \mathrm{C}$ in the presence of $5 \% \mathrm{CO}_{2}$. Subsequently, macrophages were treated with $0.25 \%$ ( $/ \mathrm{v}$ ) trypsin-EDTA (Gibco, 11560626, Fisher Scientific) for 10 min, resuspended in ice-cold FACS buffer $(0.5 \%(\mathrm{w} / \mathrm{v})$ BSA (Roche), $0.01 \%(\mathrm{w} / \mathrm{v}) \mathrm{NaN}_{3}$ in PBS), washed twice with ice-cold FACS buffer and centrifuged at $450 \times g$ for 5 min. Finally, macrophages were resuspended in FACS buffer and phagocytosis was quantified using a FACS Canto A (BD Biosciences); data were analysed using FlowJo V10 (BD Biosciences). Phagocytic activity was calculated as the relative proportion of cells that ingested at least 1 bead. Phagocytic capacity was calculated as the relative proportion of cells that ingested $\geq 3$ beads. Fold changes were calculated as phagocytic activity or phagocytic capacity of trained unstimulated macrophages relative to untrained unstimulated controls.

\section{Gene expression analysis}

Gene expression was analysed by directly lysing $1.5 \times 10^{6}$ trained or untrained macrophages ( $n=3$ independent cultures) in RLT buffer, immediately after harvest on day 12. Total RNA was isolated using the RNeasy Kit (QIAGEN) including on-column DNase treatment, according to the manufacturer's instructions, and stored at $-80^{\circ} \mathrm{C}$. Prior to CDNA synthesis, $500 \mathrm{ng}$ total RNA was treated with DNase I, Amplification Grade (Invitrogen), and CDNA was synthesized using random primers (300 ng) and Superscript III First-Strand Synthesis for RT-PCR (Invitrogen). cDNA samples were diluted in nuclease-free water prior to real-time PCR using the primers listed in Table I. Gene expression was measured by RTqPCR analysis using ABsolute qPCR SYBR Green Mix (Thermo Scientific) in a RotorGene 6000 (Corbett Research), and fluorescence data were analysed using RotorGene Analysis software version 1.7. The relative gene expression of unstimulated trained versus untrained macrophages was measured immediately after the resting period (day 12 ). The relative expression ratio $(R)$ was calculated according to the Pfaffl method (173) based on the take-off deviation of the unstimulated trained sample versus each of the unstimulated untrained controls at the same time point, and normalized relative to the $s 11$ protein of the 40 s subunit as reference gene (Table I).

Table I. Overview of RT-qPCR primers used for in the current study. cypCar numbers identify ORFs in the draft carp genome (bioproject: PRJNA73579) that were also confirmed by RNA sequencing, LHQP number refers to the accession number of the associated scaffold.

\begin{tabular}{|llll|}
\hline Primer & Forward $\left(5^{\prime}-3^{\prime}\right)$ & Reverse $\left(5^{\prime}-3^{\prime}\right)$ & $\begin{array}{l}\text { Gen Bank } \\
\text { Accession No. }\end{array}$ \\
\hline $40 s$ & CCGTGGGTGACATCGTTACA & $\begin{array}{l}\text { TCAGGACATTGAACCTCACTGT } \\
\text { CT }\end{array}$ & AB012087 \\
\hline il6a & CAGATAGCGGACGGAGGGGC & GCGGGTCTCTTCGTGTCTT & KC858890 \\
\hline il6b & GGCGTATGAAGGAGCGAGA & ATCTGACCGATAGAGGAGCG & KC858889 \\
\hline tnfaa1 & $\begin{array}{l}\text { GAGCTTCACGAGGACTAATAG } \\
\text { ACAGT }\end{array}$ & CTGCGGTAAGGGAGCAATC & AJ311800 \\
\hline
\end{tabular}


Evidence of trained immunity in teleost fish

\begin{tabular}{|llll|}
\hline tnfaa2 & CGGCACGAGGAGAAACCGAGC & $\begin{array}{l}\text { CATCGTTGTGTCTGTTAGTAAG } \\
\text { TTC }\end{array}$ & AJ311801 \\
\hline tnfab1 & $\begin{array}{l}\text { GAAGACGATGAAGATGATACC } \\
\text { AT }\end{array}$ & AAGTGGTTTCTCATCCTCAA & $\begin{array}{l}\text { cypCar_00029601, } \\
\text { LHQP01065580 }\end{array}$ \\
\hline tnfab2 & $\begin{array}{l}\text { CTTGGACGAAGCCGATGAAGA } \\
\text { C }\end{array}$ & ATCTTGTGACTGGCAAACA & $\begin{array}{l}\text { cypCar_00023012, } \\
\text { LHQP01037150 }\end{array}$ \\
\hline adpgk-1 & GGCACCACTGAACTTCT & GCGTGACCTCTGAAAACAG & $\begin{array}{l}\text { cypCar_00013411, } \\
\text { LHQP01005743 }\end{array}$ \\
\hline adpgk-2 & GCAAGCCGTGGATATTACA & GCGTGAGATGGAAGGA & $\begin{array}{l}\text { cypCar_00024520, } \\
\text { LHQP01021894 }\end{array}$ \\
\hline aldh2.1-1 & TCCAGAACTTCCCACAA & GCAGATAACCTCACCAGT & $\begin{array}{l}\text { cypCar_00011521, } \\
\text { LHQP01009285 }\end{array}$ \\
\hline aldh2.1-2 & GATTCCTGCCCCGAGTC & TTCTCCACATCCGCCTTC & $\begin{array}{l}\text { cypCar_00046381, } \\
\text { LHQP01040595 }\end{array}$ \\
\hline bpgm-1 & CGCCACCCCCCATTGAGGAGA & GCAGAGATGAGGACTGTTTG & $\begin{array}{l}\text { cypCar_00001430, } \\
\text { LHQP01009643 }\end{array}$ \\
\hline bpgm-2 & CTAAACGAGCGGCACTAC & GGGCAGTTCCTCCTTT & $\begin{array}{l}\text { cypCar_00018360, } \\
\text { LHQP01029542 }\end{array}$ \\
\hline
\end{tabular}

\section{Lactic acid production}

Production of lactic acid was measured using a lactate colorimetric assay (Kit II K627, Biovision), including an optional filtration step (Amicon 10K spin column Z677108-96EA, Sigma Aldrich), according to the manufacturer's instructions. Briefly, macrophages ( $5 \times 10^{5} /$ well, 96-well culture plate (Corning, CORN3596)) were incubated in $150 \mu \mathrm{L}$ CRPMI-1.5 for $24 \mathrm{~h}$ at $27^{\circ} \mathrm{C}+5 \% \mathrm{CO}_{2}$, after which supernatants from triplicate wells (from $n=5$ independent cultures) were pooled and filtered. For each pooled sample, a volume of $10 \mu \mathrm{L}$ was diluted $5 \mathrm{x}$ in lactate assay buffer and transferred to 96 -well plates. Subsequently, $50 \mu \mathrm{L}$ reaction mix composed of lactate substrate mix $(2 \mu \mathrm{L})$, lactate enzyme mix $(2 \mu \mathrm{L})$, and lactate assay buffer $(46 \mu \mathrm{L})$, was added to each sample and incubated for 30 minutes at RT. Optical density was measured at $450 \mathrm{~nm}$ and concentration of extracellular lactate was calculated based on a lactic acid calibration curve supplied by the manufacturer. Fold changes were calculated as lactic acid production of trained unstimulated macrophages relative to untrained unstimulated controls.

\section{Intracellular fumarate accumulation}

Accumulation of intracellular fumarate was measured using a fumarate colorimetric assay (K633, Biovision) according to the manufacturer's instructions, including an optional filtration step (Amicon 10K spin columns; as above). Briefly, macrophages ( $5 \times 10^{5} /$ well, 96-well culture plate (Corning, CORN3596)) were incubated in $150 \mu \mathrm{L}$ CRPMI- 1.5 for $24 \mathrm{~h}$ at $27^{\circ} \mathrm{C}$, after which supernatants were removed and the macrophages were lysed in $50 \mu \mathrm{L}$ fumarate assay buffer $(n=4$ independent cultures). Cell lysates from duplicate wells were pooled and filtered as mentioned above. For each pooled sample, $25 \mu \mathrm{L}$ were diluted $2 x$ in fumarate assay buffer and transferred to a 96 -wells plate. Subsequently, $100 \mu \mathrm{L}$ reaction mix composed of fumarate developer mix $(8 \mu L)$, fumarate enzyme mix $(2 \mu L)$, 
and fumarate assay buffer $(90 \mu \mathrm{L})$, was added to each sample and incubated for 30 minutes at RT. Optical density was measured at $450 \mathrm{~nm}$ and concentration of intracellular fumarate was calculated based on a fumarate calibration curve supplied by the manufacturer. Fold changes were calculated as fumarate accumulation in trained unstimulated macrophages relative to untrained unstimulated controls.

\section{Statistical analysis}

Statistical analyses were performed using SPSS (v23.0) and differences were considered significant if $p \leq 0.05$. Data presented as 'fold change' were tested for significance after log transformation. Data used for phagocytosis activity and capacity were first logit-transformed. All data, after testing for Gaussian distribution using the Shapiro-Wilk test, were analysed as paired data to eliminate interference caused by high variability between individual cultures. Optimization of training period was tested with a Friedman test on untransformed $\mathrm{NaNO}_{2}$ values $(\mu \mathrm{M})$, followed by a Dunn's post-hoc test. Comparison between untrained and trained macrophages of ROS and NO production, and comparison of constitutive gene expression was performed with a linear mixed model, followed by an LSD post-hoc test. Comparison of phagocytic activity and phagocytic capacity was performed with a paired samples t-test. Comparison of lactate production and fumarate accumulation was performed with an independent samples $T$ test. For multiple comparisons of the stimulatory effect of PGN versus laminarin, a multivariate analysis followed by LSD post-hoc test was used. 


\section{Results}

\section{Soluble peptidoglycan can be used as primary stimulus to induce trained immunity in carp macrophages}

Given that induction of trained immunity in human and mouse monocytes could be achieved via stimulation with a NOD2 ligand, we used the NOD1/2-specific soluble peptidoglycan (PGN) as a ligand to stimulate carp macrophages. To determine the optimal concentration, on day 6 of culture, macrophages were stimulated with various concentrations of PGN. Induction of NO was measured in cell supernatants after $24 \mathrm{~h}$ (Figure $2 \mathrm{~A}$ ) whereas ROS production was measured in real-time for $2 \mathrm{~h}$ and expressed as relative light units (Figure 2B). A dosedependent response was observed for both assays and based on this analysis, subsequent experiments were performed with a concentration of $1 \mu \mathrm{g} / \mathrm{mL}$ PGN as primary stimulus to induce trained immunity since this dose stimulated a robust but not maximal response.

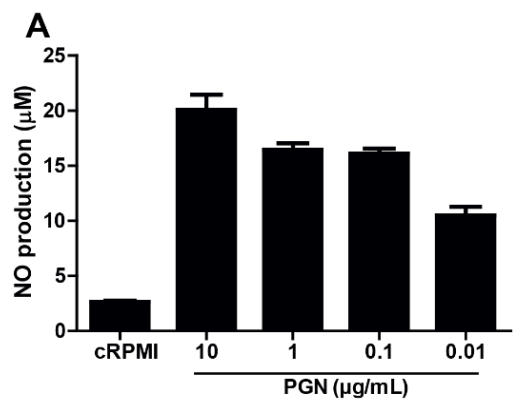

Figure 2. Peptidoglycan (PGN) induces a dose-dependent response in carp macrophages. After the differentiation period (day 6), carp macrophages were harvested, seeded in 96 well plates $\left(5 \times 10^{5}\right.$ cells/well) and stimulated with CRPMI or with the indicated concentration of PGN. A. Dosedependent analysis of PGN on Nitric oxide (NO) production. NO production was measured as $\mathrm{NaNO}_{2}$ in the supernatant

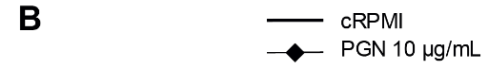

PGN $10 \mu \mathrm{g} / \mathrm{m}$
$+\quad$ PGN $1 \mu \mathrm{g} / \mathrm{mL}$
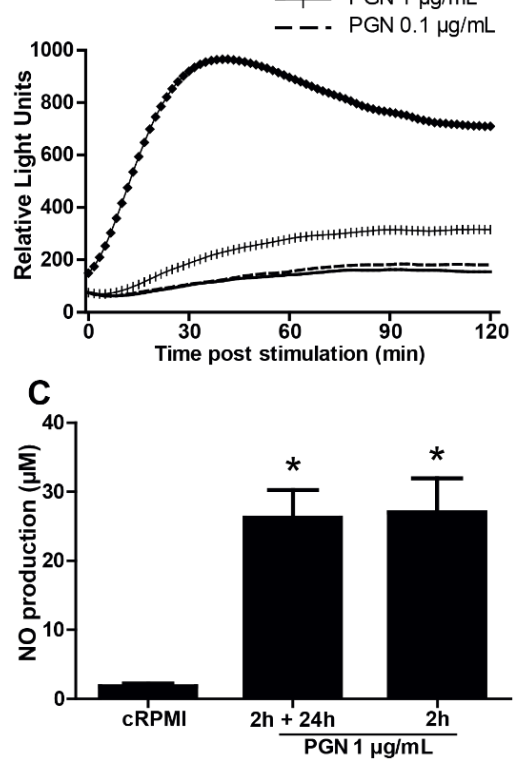
$24 \mathrm{~h}$ post-stimulation. Bars indicate mean + SD of triplicate measurements from one representative experiment out of $n=3$ performed independently. B. Dosedependent analysis of PGN on ROS production. Real-time ROS production was measured immediately following stimulation. Lines indicate acquisition of light emission in one representative experiment out of $n=3$ performed independently. C. Time course analysis of PGN on NO production. Cells were stimulated for $2 \mathrm{~h}$ by directly adding PGN or RPMI to the flask. Cells were then seeded and either incubated in CRPMI-1.5 or stimulated for additional $24 \mathrm{~h}$ with PGN $(2 \mathrm{~h}+24 \mathrm{~h})$. NO production was measured as $\mathrm{NaNO}_{2}$ in the supernatant $24 \mathrm{~h}$ postseeding. Bars indicate mean + SEM of $n=5$ independently performed experiments. Asterisk (*) indicates significant difference relative to the cRPMI control as assessed with a Friedman test, followed by a Dunn's post-hoc test (Figure 2C). 
After having determined the optimal concentration of the training stimulus, we next investigated the duration of the primary stimulation required to train macrophages. Previous studies reported that exposure for $24 \mathrm{~h}$ to the training stimulus followed by a 6-day resting period, was required for optimal training of human or mouse macrophages (168). In our case we tested whether $24 \mathrm{~h}$ or an even shorter period would be suitable to train carp macrophages. When carp macrophages were stimulated for a total of $26 \mathrm{~h}(2 \mathrm{~h}+24 \mathrm{~h})$ or for only $2 \mathrm{~h}$ with PGN, in all cases a significantly higher NO production was measured relative to the unstimulated untrained cells (RPMI) with no difference between the two durations of treatment (Figure 2C). This suggested that a stimulation period as short as $2 \mathrm{~h}$ was sufficient to stimulate carp macrophages. Altogether, soluble PGN induced a dose-dependent production of NO and ROS in carp macrophages and a $2 \mathrm{~h}$ primary stimulation with $1 \mathrm{\mu g} / \mathrm{ml}$ PGN followed by a 6 -days resting period was selected to further investigate trained immunity in carp macrophages.

\section{Trained macrophages show heightened innate immune responses}

Next, we investigated whether the response of trained macrophages differed from that of untrained macrophages upon re-stimulation with either the training stimulus (PGN, homologous) or with a different stimulus (zymosan, heterologous), (for experimental set-up see Figure 1 and $3 A$ ). Stimulation with zymosan resulted in comparable kinetics of ROS production between trained and untrained macrophages, but the production of ROS was significantly higher in trained macrophages (Figure 3B). Constitutive ROS production was not different between untrained and trained macrophages (Figure 3C, cRPMI) whereas it was significantly higher in trained macrophages exposed to zymosan or PGN (Figure 3C). Similarly, exposure to the receptor-independent stimulus PMA, resulted in a significantly higher production of ROS in trained as compared to untrained macrophages (Figure 3D). With respect to production of NO, constitutive production was not different between untrained and trained macrophages (Figure 3E, cRPMI). Exposure to zymosan, but not to LPS or PGN, resulted in a significantly higher NO production in trained as compared to untrained macrophages (Figure 3E). Phagocytic activity and phagocytic capacity of macrophages was compared between unstimulated trained and unstimulated untrained macrophages. A significantly higher number of cells with internalized beads as well as higher number of beads per cell, was observed for trained as compared to untrained macrophages, indicative of a heightened phagocytic activity as well as phagocytic capacity of trained macrophages (Figure 3F). Altogether, measurement of ROS and NO production as well as measurement of phagocytosis showed heightened innate immune functions of trained carp macrophages. 


\begin{tabular}{|ccc|}
\hline & Differentiation period & Resting period \\
\hline Day 0 & Day 6 & Day 12 \\
& $\begin{array}{c}\text { Macrophages } \\
\text { No training } \\
\text { PGN training }\end{array}$ & $\begin{array}{c}\text { Trained macrophages } \\
\text { Untrained } \\
\text { Thained }\end{array}$ \\
\hline
\end{tabular}
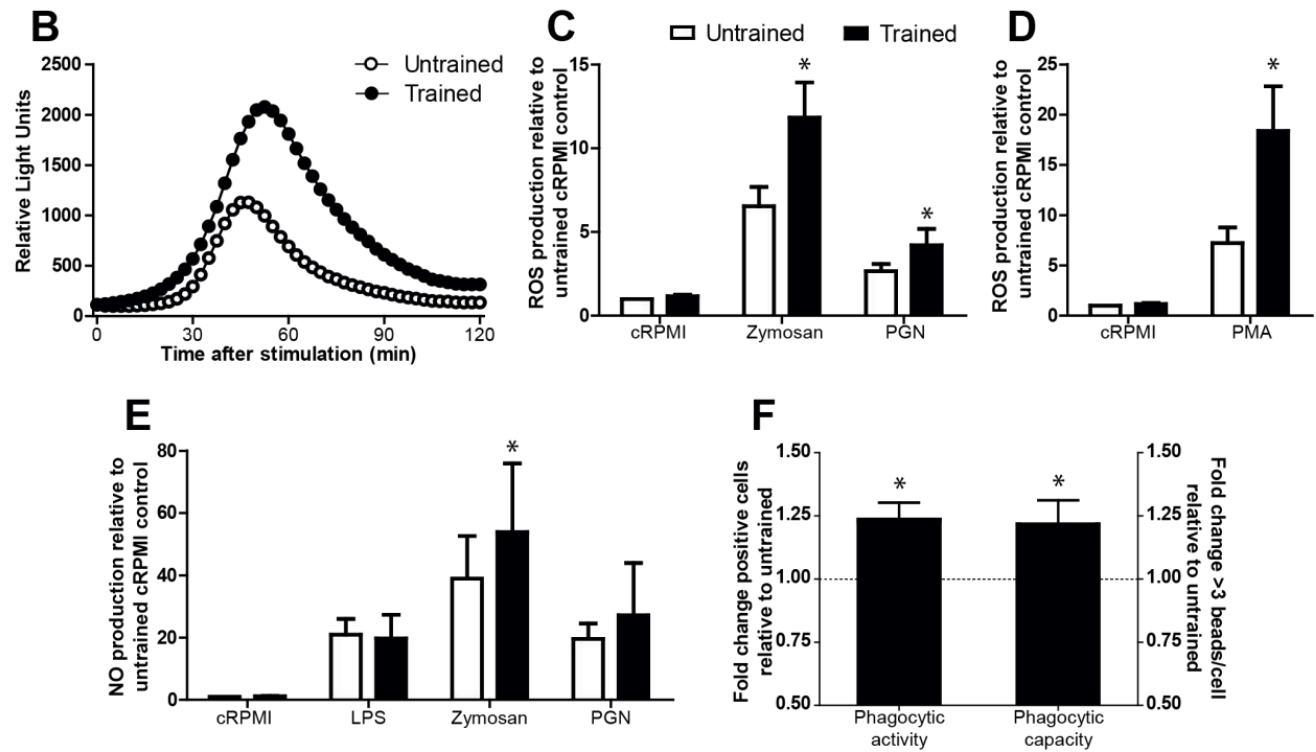

Figure 3. Trained carp macrophages show increased innate immune functions. $A$. Experimental in vitro set-up. Head kidney leukocytes were differentiated into macrophages for $6 \mathrm{~d}$. On day 6 , macrophages were stimulated with $1 \mu \mathrm{g} / \mathrm{ml}$ PGN (trained) or vehicle control (untrained), followed by a resting period of 6 days. On day 12, trained or untrained macrophages were harvested and used for subsequent analyses. B-D. Trained or untrained macrophages were seeded in 96 -well plates $\left(5 \times 10^{5}\right.$ cells/well) and stimulated with cRPMI, zymosan $(250 \mu \mathrm{g} / \mathrm{mL})$, PGN $(10 \mu \mathrm{g} / \mathrm{mL})$ or PMA $(1 \mu \mathrm{g} / \mathrm{mL})$. B. Real-time ROS production was measured immediately following stimulation. Lines indicate acquisition of light emission measured in one representative experiment out of $n=11$ independently performed experiments. C-D. Total ROS production relative to unstimulated, untrained macrophages (CRPMI); bars indicate mean + SEM of $n=11$ experiments performed independently. $E$. Trained or untrained macrophages were seeded in 96 -well plates $\left(5 \times 10^{5}\right.$ cells/well) and stimulated with cRPMI, LPS $(50 \mu \mathrm{g} / \mathrm{mL})$, zymosan $(250 \mu \mathrm{g} / \mathrm{mL})$ or PGN $(10 \mu \mathrm{g} / \mathrm{mL})$. NO production was measured as $\mathrm{NaNO}_{2}$ in the supernatant $15 \mathrm{~h}$ post-stimulation. NO production is expressed relative to unstimulated untrained macrophages (CRPMI); bars indicate mean + SEM of $n=7$ experiments performed independently. $F$. Unstimulated trained or unstimulated untrained macrophages were analysed for their phagocytic activity (left $y$-axis) and phagocytic capacity (right $\mathrm{y}$-axis) after $2 \mathrm{~h}$ incubation with fluorescent beads ( 1 cell:10 beads). Fold change is expressed relative to the corresponding untrained cells; bars indicate mean + SEM of $n=6$ experiments performed independently. Asterisk $\left.{ }^{*}\right)$ indicates significant difference relative to the corresponding untrained sample as assessed by a linear mixed model one-way ANOVA, followed by a LSD post-hoc test (Figure $3 \mathrm{C}-\mathrm{E}$ ) or paired samples t-test (Figure 3F). 


\section{Trained macrophages show immune and metabolic reprogramming}

Studies on trained human PBMC revealed a differential expression of IL6 and TNFa in response to microbial stimulation, which introduced metabolic reprogramming towards glycolysis as an important mechanism underlying trained immunity. Therefore, after having assessed that trained macrophages display heightened responses to stimulation (Figure 3), we also analysed in more details whether differences exist in the constitutive expression of inflammatory or metabolic genes between unstimulated trained and unstimulated untrained macrophages. Trained macrophages showed moderate but significantly increased expression of inflammatory genes, including il6 and tnfa, each present in multiple copies (paralogues) in carp (Figure 4A). A trend towards increased il $\beta$ expression in trained macrophages was also observed ( $p$ value $=0.06$ ), whereas il10 and inosb expression remained unchanged (data not shown). With respect to the expression of metabolic genes, trained macrophages showed significantly increased expression of specific paralogs of adpgk, aldh2.1 and bpgm (Figure 4B), suggestive of activation of the glycolysis pathway compared to untrained cells. Further evidence of metabolic reprogramming in unstimulated trained macrophages towards glycolysis was obtained upon measurement of extracellular lactate concentrations and intracellular accumulation of the metabolite fumarate. Trained macrophages showed significantly higher lactate values and a trend ( $p$ value = 0.077 ) towards higher accumulation of fumarate as compared to untrained cells (Figure 4C). Altogether, these data suggest that markers of immune and metabolic reprogramming were increased in trained macrophages. 

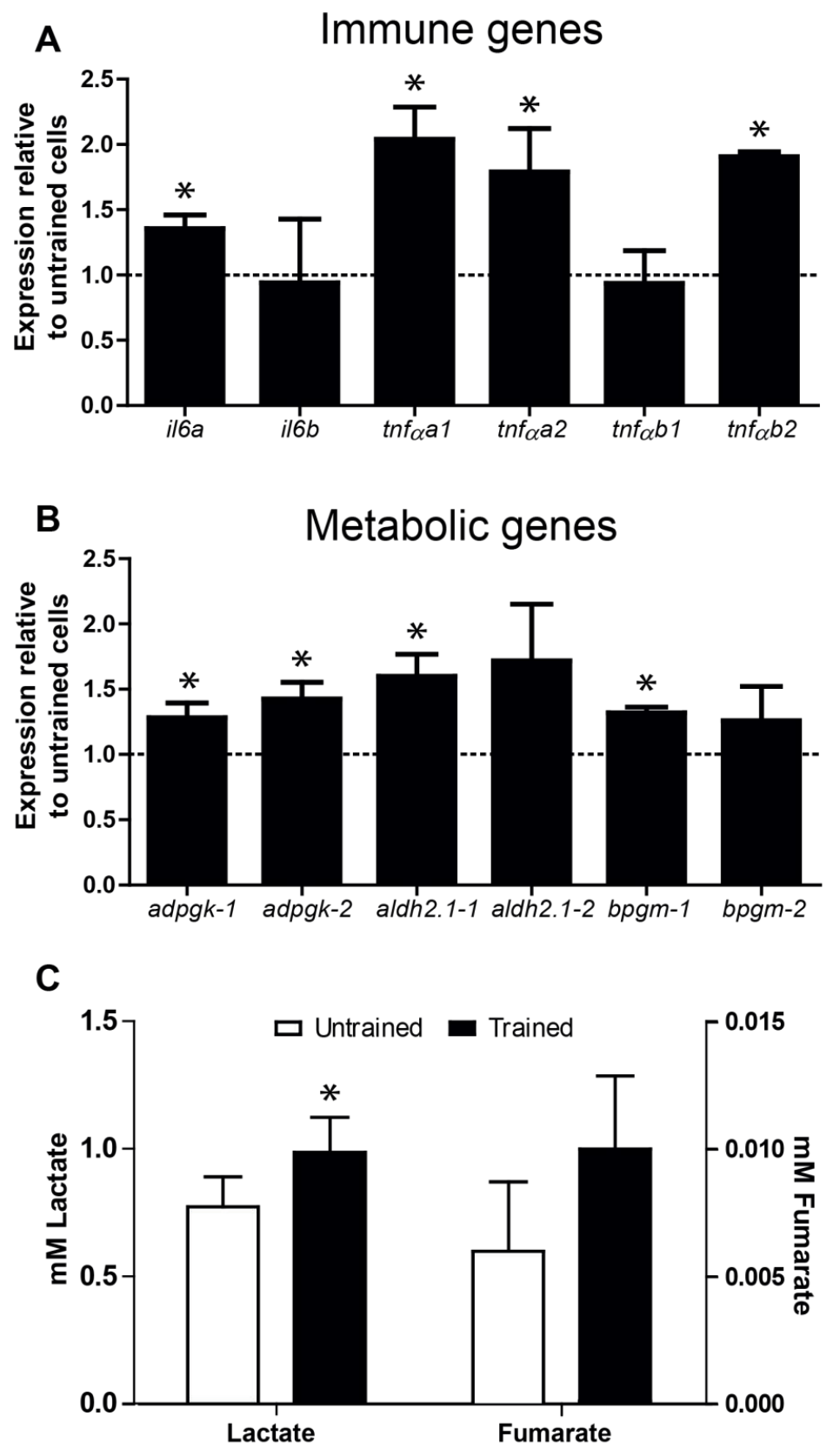

Figure 4. Trained carp macrophages show immune and metabolic reprogramming. Trained or untrained macrophages were obtained as described in figure 3. A-B. Gene expression of il6 and tnfa paralogues (A) or selected metabolic genes and their paralogues (B) in unstimulated trained macrophages relative to unstimulated untrained controls; bars indicate mean + SEM of $n=3$ experiments performed independently. C. Lactate and Fumarate accumulation. Unstimulated trained or unstimulated untrained macrophages were seeded in 96 -well plates ( $5 \times 10^{5}$ cells/well) and accumulation of extracellular lactate and intracellular fumarate was measured $24 \mathrm{~h}$ later; bars indicate mean + SEM of $n=5$ (lactate) and $n=4$ (fumarate) experiments performed independently. Asterisk $(*)$ indicates significant difference relative to the corresponding untrained sample as assessed by a linear mixed model one-way ANOVA, followed by a LSD post-hoc test (Figure 4A-B) or independent samples test (Figure 4C). 


\section{Soluble $\beta$-glucan laminarin is a potent training stimulus}

In human PBMC, stimulation with BCG or $\beta$-glucans could induce trained immunity. We therefore investigated whether besides PGN, also the soluble $\beta$-glucan laminarin could induce trained immunity in carp macrophages (Figure 5). Training with laminarin, but not PGN, led to a marginal but significant increase in constitutive production of ROS as compared to untrained cells (CRPMI). Training with laminarin and subsequent exposure to zymosan or PMA, resulted in significantly heightened production of ROS, comparable to macrophages trained with PGN. Altogether, measurement of ROS production proved particularly informative to identify ligands able to train carp macrophages and revealed that not only NOD-ligands but also $\beta$-glucans can serve as suitable training stimuli of fish macrophages.

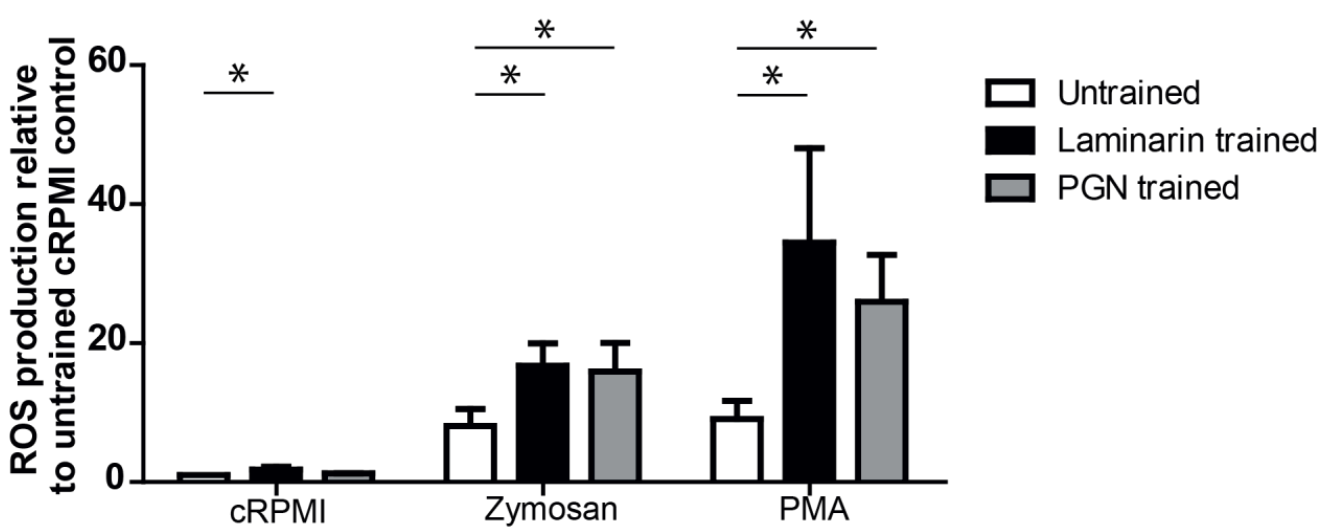

Figure 5. Exposure to laminarin leads to trained immunity in carp macrophages. On day 6 , macrophages were trained with laminarin $(20 \mu \mathrm{g} / \mathrm{mL})$ or PGN $(1 \mu \mathrm{g} / \mathrm{mL})$ or left untrained (vehicle control), followed by a resting period of 6 days. On day 12 , trained or untrained macrophages were harvested and seeded in 96 -well plates $\left(5 \times 10^{5}\right.$ cells/well). ROS production was measured immediately following stimulation with either CRPMI, zymosan $(250 \mu \mathrm{g} / \mathrm{mL})$ or PMA $(1 \mu \mathrm{g} / \mathrm{mL})$ and expressed relative to the unstimulated untrained control (cRPMI); bars indicate mean + SEM of $n=6$ experiments performed independently. Asterisk $(*)$ indicates significant differences relative to the corresponding untrained sample as assessed by a multivariate analysis, followed by LSD post-hoc test. 


\section{Discussion}

In the present study, we adapted a well-described in vitro culture system of head kidney-derived macrophages to investigate conservation of trained immunity in teleost fish. A $2 \mathrm{~h}$ in vitro exposure to a soluble NOD-specific ligand or to soluble $\beta$ - $(1,3 / 1,6)$-glucan resulted in carp macrophages that displayed typical features of trained immunity for a period of at least six days. The use of soluble ligands allowed for thorough washing and removal of traces of ligands, a procedure we considered crucially important to exclude continuous re-stimulation of macrophages. Typical features of trained macrophages of carp included heightened phagocytosis and inflammatory responses following stimulation with (homologous or heterologous) microbial stimuli. The inflammatory profile displayed heightened production of reactive oxygen or nitrogen species, increased constitutive gene expression of selected immune and metabolic genes as well as an increased constitutive lactate production, illustrative of a metabolic shift. Measurement of the production of reactive oxygen species proved particularly informative to identify ligands able to train carp macrophages.

The present study provides in vitro evidence for conservation of several key features of trained immunity in macrophages of carp, a representative teleost fish, but provides no in vivo evidence for trained immunity. However, vaccination with BCG, a known stimulant of trained immunity in mice/human monocytes, has already been shown to provide cross-protection against Mycobacterium sp. infections in several fish species (149-151) including zebrafish (174), a well-known animal model closely related to carp. The non-specific protection provided by BCG injection suggests that also in fish, in vivo evidence for trained immunity already exists. In addition, zebrafish i.p.-injected with $\beta$-glucans and subsequently challenged with spring viremia of carp virus (SVCV), either showed a significant but minor increase in survival at 7 but not 35 days post-treatment (158), or a clear increase in survival at 14 days post treatment (175). Thus, relatively long-lived effects of immune stimulants not specifically associated with anti-viral immunity, provide at least circumstantial in vivo evidence for trained immunity in zebrafish. All of the above-described studies clearly indicate the complexity of in vivo experiments, not unique to experiments in fish, in which it is not easy to exclude the involvement of adaptive immunity and the possibility of continuous stimulation as opposed to a single training event, a clear advantage of our in vitro system based on soluble ligands and rigorous washing steps.

Zebrafish could prove especially informative for in vivo studies on trained immunity owing to the availability of $\mathrm{rag}^{-/-}$strains. Increased survival of $\mathrm{rag}^{-/-}$zebrafish upon lethal challenge with Edwardsiella ictaluri eight weeks post-exposure to an attenuated non-virulent strain of this bacterium (152) could be considered in vivo evidence of trained immunity, although persistence of attenuated bacteria and absence of non-specific cross-protection against another bacterium, Yersinia ruckeri, could not fully exclude the involvement of other protective mechanisms. Only recently, $\mathrm{rag}^{-/}$zebrafish were shown to exhibit a constitutively heightened 
innate immune activity, characterized by an increased antiviral state and associated resistance to a viral challenge with SVCV (157). In the latter study, also NK cell markers such as cd8 and nklysin were constitutively higher expressed in $\mathrm{rag}^{-/-}$than in wild type zebrafish. This observation is of particular interest in view of the BCG-induced $(176)$ and virus-induced $(177,178)$ memory-like NK cells, recently highlighted as cell types associated with trained immunity in mammals. It could thus be relevant to further study NK-like cells in relation to virus-induced trained immunity in the $\mathrm{rag}^{-/}$zebrafish model, and to explore genes associated with an antiviral state as novel markers for trained immunity.

Teleost fish (e.g. carp, zebrafish) are poikilotherms, which allows for manipulation of temperature and thus allows for studies on temperature-associated effects on trained immunity in vitro and in vivo. Adaptive immunity, more than innate immunity, is considered sensitive to temperature change, reflected by reduced IgM serum concentrations and suppression of $\mathrm{T}$ cell responses at lower temperatures $(179,180)$. Thus, the use of poikilothermic animals opens the possibility to 'knockdown' adaptive immune responses in animals for which $\mathrm{rag}^{-/}$strains are not available, and to study, in vivo, aspects of trained immunity, including the duration of cross-protection against a secondary infection in a $\mathrm{T}$ - and B-lymphocyte independent manner. Temperature-mediated 'knock-down' of adaptive immune responses in teleost fish might thus allow to unravel mechanisms underlying longlived protection likely mediated by innate immune cells that remain active also at lower temperatures.

One of the determining and underlying mechanisms of trained immunity is based on long-lived epigenetic modifications that persist even after removal of the training stimulus (as reviewed by: (181)). For example, histone modifications such as trimethylation of $\mathrm{H} 3 \mathrm{~K} 4$ and mono acetylation of $\mathrm{H} 3 \mathrm{~K} 27$ have been associated with $\beta$-glucan- induced trained immunity of human PBMC. These epigenetic changes were strongly correlated with differential gene expression induced by $\beta$ glucan training in monocytes (58). In the same study, comparable epigenetic changes were observed in peritoneal macrophages isolated from C. albicansinfected mice, linking the observed in vitro histone modifications to an in vivo model of trained immunity. The onset of these epigenetic modifications is strongly connected to metabolic reprogramming, and dependent on specific metabolites such as fumarate and mevalonate $(59,60)$. In our in vitro model we investigated epigenetic modifications in trained macrophages by measuring (increased) constitutive expression of several immune- and glycolysis-related genes, and a metabolic shift from oxidative phosphorylation towards glycolysis. To study in more detail epigenetic modifications underlying trained immunity in fish, future studies could build on, for example, a recent chromatin immunoprecipitation (ChIP) sequencing study performed on SVCV-infected zebrafish (182). Another interesting aspect of trained immunity that could be studied in vivo, at least in oviparous fish, is transgenerational epigenetic reprogramming. Since primordial F2 germ cells are not present upon exposure of FO individuals to potential training stimuli, one less generation is needed to prove transgenerational effects in 
oviparous fish (183). In conclusion, the constitutive antiviral state of $\mathrm{rag}^{-/-}$ zebrafish, the cold-blooded nature of teleost fish allowing "natural" knockdown of the adaptive immune system, and the suitability of oviparous fish for transgenerational experiments, all provide arguments in favour of studying conserved but also unique aspects of trained immunity in teleost fish.

\section{Acknowledgements}

Raphael Barbetta de Jesus and Fabiana Pilarski are greatly acknowledged for their discussions on the potential impact of trained immunity on aquaculture practise. Cassandra van Doorn is greatly acknowledged for her contribution to the initial cell culture optimization. 



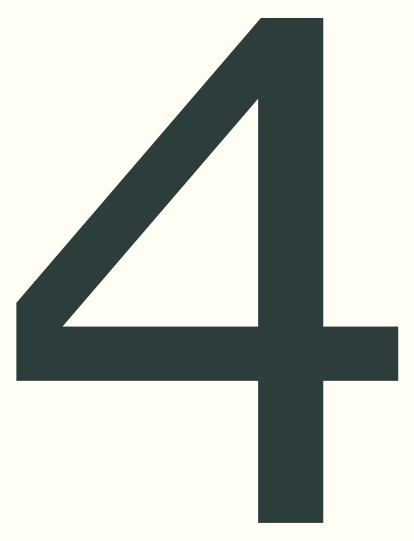

\section{$\beta$-glucan immuno-modulation in common carp intestine: a role for microbiota and its metabolite}

Jules Petit, Irene de Bruin, Sylvia Brugman, Wilbert F. Pellikaan, Maria Forlenza, Geert F. Wiegertjes

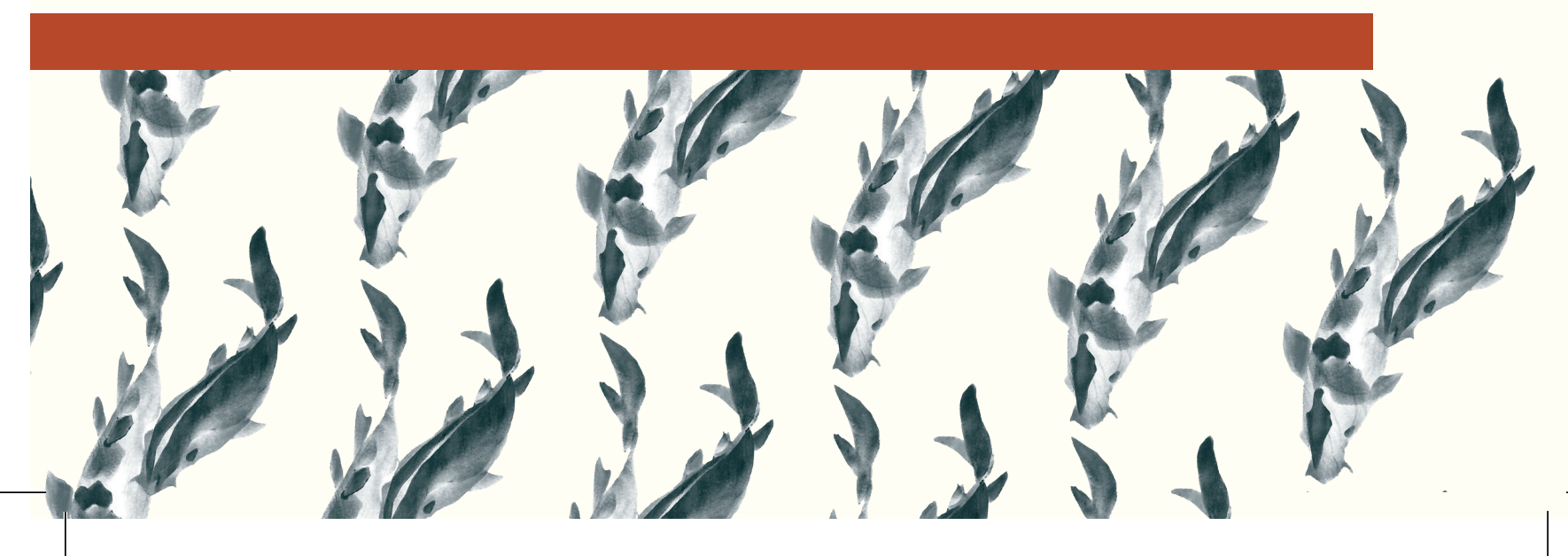




\section{Abstract}

Dietary supplementation of fish with $\beta$-glucans has been widely associated with immunomodulation and commonly accepted as beneficial for fish health. However, to date the exact mechanisms of immunomodulation by $\beta$-glucan supplementation in fish are still largely unknown. In mammals a clear relation has been observed between high fibre diets and immunomodulation via intestinal microbiota and its metabolites. In this study, we first described the normal microbiota of common carp intestine by 165 rRNA sequencing. Based on the abundance of the genus Bacteroides, well known for their capacity to degrade and ferment carbohydrates, we hypothesized that common carp intestinal microbiota could ferment dietary $\beta$-glucans. Indeed, two different $\boldsymbol{\beta}$ glucan preparations (curdlan and MacroGard ${ }^{\circledR}$ ) were both fermented in vitro albeit with distinct fermentation dynamics and significant differences in production of short chain fatty acids (SCFA). MacroGard ${ }^{\circledR}$ more than curdlan lead to production of propionate, a SCFA with immunomodulatory properties. Single oral gavage of MacroGard ${ }^{\circledR}$ in ninemonth-old carp significantly altered the intestinal microbiota. At seven days post-treatment, increased abundance of a member of the family Rhodocyclaceae was observed, which contains genera known to synthesize propionate using unusual transcarboxylase enzymes. Coinciding with the shift in microbial composition, an overall immunomodulation in the intestine could be observed as inhibition of expression of several pro-inflammatory genes (il1 $\beta, i l 6, t n f a)$. Based on our data, we discuss the possibility that fermentation of MacroGard ${ }^{\circledR}$ by specific bacteria, part of the normal microbiota of common carp intestine, can lead to a shift in microbial composition and associated production of the SFCA propionate, the increased presence of which could possibly explain (part of) $\beta$-glucan-induced immunomodulatory effects. 


\section{Introduction}

Effects of immunomodulation by $\beta$-glucans have been widely studied in teleost fish. Regardless of administration route or fish species, $\beta$-glucans often are associated with immunomodulatory effects and increased resistance to both viral and bacterial infections (reviewed by: (19-21). Dietary supplementation of $\beta$ glucans may be frequently applied in aquaculture, but definitive mechanisms explaining the observed immunomodulatory effects of $\beta$-glucans in the intestine have yet to be described for fish.

In mammals, direct recognition of $\beta$-glucans is mediated by at least the C-type lectin receptor (CLR) Dectin-1 (27-29), whereas in invertebrates $\beta$-glucans can be recognized by $\beta$-glucan binding proteins $(184)$. For both, mammals $(43,185)$ and invertebrates $(89,184)$, several non-exclusive pathways play a downstream role in the response to $\beta$-glucans, often mediating activation of the transcription factor NF-KB (38). Although in fish genomes no clear homologue of Dectin-1 could be identified so far $(28,42)$, in recent studies we could activate carp macrophages with curdlan $(41,138)$, considered a Dectin-1-specific $(1,3)-\beta$-glucan stimulus. We therefore proposed that immuno-modulatory effects of $\beta$-glucan in carp macrophages could include regulation of a downstream signalling pathway typical of CLR activation, and confirmed our hypothesis by pathway analysis of differentially expressed genes (DEGs), (41). Among the regulated genes were card9 and $b c / 10$, both members of the signalling complex shown to be downstream of Dectin-1. Not only did we observe regulation of the CLR signalling pathway, several candidate receptors could be identified based on regulation of expression upon stimulation with $\beta$-glucans, or based on the conservation of $\beta$-glucan binding motifs in their predicted translations to protein (41). As there is clear evidence for presence of macrophages and other antigen-sampling cells in the intestine (93, $186,187)$, it is possible that recognition of $\beta$-glucans in the intestine occurs via a direct recognition mechanism of $\beta$-glucan receptors. Another mechanism could be indirect and rely on prebiotic effects inducing a shift in intestinal microbiota which would influence the microenvironment of the intestine. These mechanisms should not necessarily be exclusive.

The intestinal microbiota is of well-known importance to the host, for example via degradation and fermentation of non-starch polysaccharides and/or resultant production of metabolites (188-190). Several studies have shown the ability of fish microbiota to degrade carbohydrates, showing growth of specific intestinal bacterial monocultures on different carbohydrates among which $\beta$-glucan (191), and observing in vitro fermentation of several carbohydrates and production of short chain fatty acids (SCFAs) by intestinal microbes of common carp (Cyprinus carpio) or Nile tilapia (Oreochromis niloticus) $(192,193)$. In a comparison of the fermentability of different carbohydrates and produced SCFAs between Nile tilapia and European sea bass, both their microbiota were found capable of fermenting carbohydrates but with differing end-product profiles (194). Effects of $\beta$-glucan supplementation on the composition of the intestinal microbiota are not well 
documented, although several studies did observe differences due to dietary $\beta$ glucan supplementation. In common carp, two weeks feeding with $\beta$-glucanenriched diets induced shifts in the normal intestinal microbiota, manifested either as a reduction in species richness and absolute number of operational taxonomic units (OTUs), (79), or as an increase in microbial diversity (126). In seabass, four weeks feeding with $\beta$-glucan-enriched diets induced a shift towards more Methylobacterium (127), whereas in rainbow trout and feral common carp, 8 weeks feeding with yeast-derived probiotics rich in $\beta$-glucans induced a shift towards more lactate bacteria $(78,195)$. Overall, possibly owing to differences in diet formulation, sampling, microbial analysis and fish species, a consistent and distinct shift in intestinal microbial composition induced by $\beta$-glucans remains to be discerned.

We studied the role of $\beta$-glucan as prebiotic and hypothesized that common carp normal intestinal microbiota would be able to ferment $\beta$-glucans. We further hypothesized that, as a result of this fermentation process, in vivo treatment of carp with $\beta$-glucans would induce a shift in intestinal microbial composition and might result in a shift in production of short chain fatty acid (SCFA) metabolites. Finally, we hypothesized that this series of processes would contribute to the commonly observed immunomodulation of the host. To test our hypotheses, we first characterised the normal, active intestinal microbiota of common carp by $16 \mathrm{~S}$ rRNA transcript sequencing. We also analysed the SCFA profile generated during in vitro fermentation and noticed $\beta$-glucan-dependent shifts in SCFA production. Last but not least, common carp were treated with a single oral gavage with $\beta$ glucans to analyse in vivo effects of $\beta$-glucans on intestinal microbiota and effects on regulation of local gene expression. Our data suggest that dietary $\beta$-glucan administration may have indirect immunomodulatory effects accompanied by a shift in intestinal microbiota and associated shift in SCFA profile, of which the interconnection may require further investigation.

\section{Material and Methods}

\section{Animals}

European common carp (Cyprinus carpio carpio L.) of the R3 $\times$ R8 strain were used, which originated from a cross between the Hungarian R8 strain and the Polish R3 strain (169). Carp were bred and raised in the aquatic research facility of Wageningen University at $20-23^{\circ} \mathrm{C}$ in recirculating UV-treated water and fed pelleted dry food (Skretting, Nutreco) twice daily. All experiments were performed with the approval of the animal experiment committee of Wageningen University (DEC number 2015098).

\section{Collection of intestinal contents for 16s rRNA profiling}

Nine-month-old fish were anaesthetized in $0.3 \mathrm{~g} / \mathrm{L}$ Tricaine Methane Sulfonate (TMS) (Crescent Research Chemicals, Phoenix, USA) in aquarium water buffered 
with $0.6 \mathrm{~g} / \mathrm{L}$ sodium bicarbonate and bled via the caudal vein. The third segment of the intestine was isolated and intestinal content of the third segment was collected by scraping, snap-frozen in liquid nitrogen and stored at $-80^{\circ} \mathrm{C}$. A tissue sample of the third segment of the intestine was snap-frozen in liquid nitrogen and stored at $-80^{\circ} \mathrm{C}$.

\section{Total bacterial RNA isolation}

Prior to isolation of RNA from intestinal content, all samples were weighed. Total RNA from intestinal content was isolated using the RNeasy PowerMicrobiome Kit (Qiagen, 26000-50), following the manufacturer's instructions. Agarose gel electrophoresis and a ND1000 spectrophotometer (NanoDrop Technologies, Wilmington, DE, USA) were used to control RNA yield and quality. RNA samples were stored at $-80^{\circ} \mathrm{C}$ until further use.

\section{S rRNA profiling}

Total community analyses were performed on initially five replicates per treatment similar as described for (196). Total community RNA was used for amplification and sequencing of the $16 \mathrm{~S}$ rRNA, targeting the variable V3-V4 regions resulting in amplicons of approximately $\sim 460 \mathrm{bp}$. Illumina 16S rRNA gene amplicon libraries were generated and sequenced at BaseClear (Leiden, the Netherlands). In short, barcoded amplicons from the V3-V4 region of $16 \mathrm{~S}$ rRNA genes were generated using a 2-step approach. Ten ng genomic RNA was used as template for the OneStep RT-PCR kit (QIAGEN $R$ ) employed according to manufacturer's instructions with a total volume of 50 ul using the $341 \mathrm{~F}\left(5^{\prime}-\right.$ CCTACGGGNGGCWGCAG-3') and the 785R (5'-GACTACHVGGGTATCTAATCC- $3^{\prime}$ ) primers appended with Illumina adaptor sequences. Control PCR reactions were performed alongside each separate amplification without addition of template. PCR products were purified using AMPure XP beads according to manufacturer's instructions and the size of the PCR products were checked on Fragment analyzer (Advanced Analytical) and quantified by fluorometric analysis. Purified PCR products were used for the 2nd PCR in combination with sample-specific barcoded primers (Nextera XT index kit, Illumina). Subsequently, PCR products were purified, checked on a Fragment analyzer (Advanced Analytical) and quantified, followed by multiplexing, clustering, and sequencing on an Illumina MiSeq with the paired-end (2x) 300 bp protocol and indexing. The sequencing run was analyzed with the Illumina CASAVA pipeline ( $v 1.8 .3$ ) with demultiplexing based on samplespecific barcodes. The raw sequencing data produced was processed removing the sequence reads of too low quality (only "passing filter" reads were selected) and discarding reads containing adaptor sequences or PhiX control with an in-house filtering protocol. A quality assessment on the remaining reads was performed using the FASTQC quality control tool version 0.10.0. 


\section{Bacterial community analyses}

The RDP extension to PANDASeq (197) named Assembler (198) was used to merge paired-end reads with a minimum overlap of $10 \mathrm{bp}$ and at least a PHRED score of 25. Primer sequences were removed from the per sample FASTQ files using FLEXBAR version 2.5 (199). Sequences were converted to FASTA format and concatenated into a single file. All reads were clustered into OTUs using the UPARSE strategy by dereplication, sorting by abundance with at least two sequences and clustering using the UCLUST smallmem algorithm (200). These steps were performed with VSEARCH version 1.0.10 (201), which is an opensource and 64-bit multithreaded compatible alternative to USEARCH. Next, chimeric sequences were detected using the UCHIME algorithm (202) implemented in VSEARCH. All reads before the dereplication step were mapped to OTUs using the usearch_global method implemented in VSEARCH to create an OTU table and converted to BIOM-Format 1.3.1 (203). Finally, taxonomic information for each OTU was added to the BIOM file by using the RDP Classifier version 2.10 (204). All steps were implemented in a Snakemake workflow (205), which is available on Github https://github.com/nioo-knaw/hydra/tree/1.3.3.

For downstream analysis we took the obtained OTU table and prepared a "filtered table" using QIIME (1.9.1) custom scripts (206). First, we extracted from the OTU table the Bacteria domain using the command split_otu_table_by_taxonomy.py. Next, we discarded singletons, doubletons, Chloroplast and Mitochondria sequences using the command filter_otus_from_otu_table.py. With the "filtered_OTUtable", we first calculated the alpha diversity. Using the command alpha_rarefaction.py, the OTU table was rarefied to counts up to 3975 reads. The reason to use this value was because this was the lowest sequencing depth obtained from a sample. To calculate the diversity indexes, we used the alpha_diversity.py and alpha_rarefaction commands, obtaining Shannon, Observed OTUs and Chao1 metrics and the compare_alpha_diversity.py for calculating the statistical difference between the treatments. For the Beta diversity, the unrarefied "filtered_OTUtable" was first normalized using the R package metagenomeSeq (v.1.12) (207, 208). We used a cumulative-sum scaling (CSS) method to avoid the biases generated with current sequencing technologies due to uneven sequencing depth (209) accompanied by fitZIG contrasts. With the normalized OTU table Bray-Curtis dissimilarity matrices were calculated and used to build Principal Coordinate with Phyloseq package (v.1.10) (210). The bubble plots were drawn with TreeMap@). Firstly, using the command alpha_rarefaction.py, the OTU table was rarefied to counts up to 3975 reads. Subsequently, random forest analysis was performed, followed by the Boruta feature selection (211) on rarefied data using 1000 trees with $\mathrm{R}$ packages randomForest v4.6-7 and Boruta v3.0, respectively. Subsequently, heatmaps were constructed based on z-score transformed (count-average count/standard deviation of counts) functional annotations to improve normality and homogeneity of variances and using the pheatmap R package (212). 


\section{In vitro gas production technique}

In vitro gas production technique is an in vitro batch culture method that can be used to assess the potential fermentability of monogastric feed ingredients (194, $213,214)$. With this technique, an inoculum is prepared under strictly anaerobic conditions using freshly collected intestinal contents. The inoculum is incubated with a fermentable substrate of choice and with medium to support bacterial growth. Subsequently, accumulating gas during fermentation is measured so that a picture of the kinetics of fermentation is obtained. At the end of the fermentation period, samples are taken from the fermentation fluid for measurement of fermentation end-products and substrate utilization (215).

\section{Collection of intestinal contents for in vitro fermentation}

Naive carp were anaesthetized with $0.3 \mathrm{~g} / \mathrm{l}$ Tricaine Methane Sulfonate (TMS) (Crescent Research Chemicals, Phoenix, USA) in aquarium water buffered with 0.6 $\mathrm{g} / \mathrm{L}$ sodium bicarbonate and bled via the caudal vein. Subsequently, fish were put on ice and the intestine, without bulbus, was removed. The content of the intestine was collected in pre-weighed plastic tubes that were filled with $\mathrm{CO}_{2}$ to ensure anaerobic conditions. Intestinal content of ten fish was pooled and five independent pools were used.

\section{Substrates for in vitro fermentation}

Four different substrates were used for in vitro gas production analyses, glucose, (D-glucose monohydrate; Merck, Darmstadt, Germany), PBS (Cell culture grade, Lonza), Curdlan ( $\beta-(1,3)$-glucan extracted from Alcaligenes faecalis, SigmaAldrich) and MacroGard ${ }^{\mathrm{TM}}$ (a cell wall preparation of $S$. cerevisiae comprising at least $60 \% \beta$-glucan (Zilor, São Paulo, Brazil)). Glucose was included as a readily fermentable substrate for comparative purposes (194).

\section{Inoculum preparation and measurement of cumulative gas production}

Pooled intestinal content was immediately transported to the laboratory after collection where it was weighed and transferred to a beaker. The contents of the beaker were stirred and flushed with a constant gentle stream of $\mathrm{CO}_{2}$. Pre-warmed $\left(25^{\circ} \mathrm{C}\right)$ anaerobic, sterile saline $(9 \mathrm{~g} / \mathrm{L} \mathrm{NaCl})$ was added in a ratio of $1: 5(\mathrm{~W} / \mathrm{V})$ to ensure sufficient amount of inoculate. The diluted material was homogenized using a vortex mixer and strained through a double layer of cheese cloth with 16 threads per $\mathrm{cm}$ in both directions. From the resulting inoculate, $5 \mathrm{~mL}$ was then dispensed into a pre-warmed $300 \mathrm{~mL}$ fermentation bottle, containing $0.5 \mathrm{~g}$ substrate and 82 $\mathrm{mL}$ of medium. Three replicate bottles for each substrate per inoculate were used. The medium consisted of a basal solution containing micro-nutrients required by the microbial population for growth, a bicarbonate solution, a vitamin/phosphate solution and a reducing agent. The composition of the medium is described in detail by (216). Subsequently, bottles were immediately attached to an automated gas production system (217). Within this system, pressure sensors detect a fixed pressure, after which a computer software program allows opening of a valve to 
release gas, the time at which this occurred was recorded. Bottles were incubated for 168 hours at $25^{\circ} \mathrm{C}$, equal to the body temperature of carp.

Curve fitting and statistics of cumulative gas production

A monophasic model as described by (218) was fitted to the profile of the cumulative gas production of each fermentation bottle according to the equation $\mathrm{G}=\mathrm{A} /(1+(\mathrm{C} / \mathrm{t}) \mathrm{B})$, where $\mathrm{G}$ is the total millilitre gas produced per gram organic matter $(\mathrm{OM})$ at time $\mathrm{t}$; $A$ is the asymptotic gas production $(\mathrm{mL} / \mathrm{g} \mathrm{OM}) ; B$ is the switching characteristic of the curve; $\mathrm{C}$ is the half time (time at which half of the asymptote is reached) and $t$ is the time ( $h)$.

The maximum rate of gas production (Rmax) and the time at which it occurs (Tmax), were calculated according to (219) as

$$
\begin{aligned}
& R_{\max }=\left(A\left(C^{B}\right) B\left(T_{\max }{ }^{(-B-1)}\right)\right) /\left(1+\left(C^{B}\right)\left(T_{\max }{ }^{(-B)}\right)\right)^{2} \text { and } \\
& T_{\max }=C\left(((B-1) /(B+1))^{(1 / B)}\right)
\end{aligned}
$$

For each bottle, curve fitting was done using the nonlinear least-squares regression procedure NLIN (SAS Inst. Inc., Cary, NC, USA). One-way analysis of variance using the GLM procedure of SAS (SAS Inst. Inc.) was used to test the effect of substrate on gas production parameters and fermentation end-products. The average of replicate bottles per substrate per inoculum was considered as the experimental unit. The effect of replicate bottles was tested separately and was not significant for any of the parameters. It was therefore excluded from the model and thus became part of the error term.

\section{Sampling and analyses of fermentation liquid}

At the end of the incubation period, $\mathrm{pH}$ of the fermentation fluids was recorded and samples were collected for analysis of ammonia $\left(\mathrm{NH}_{3}\right)$, SCFAs and lactate. Analysis of $\mathrm{NH}_{3}$ and SCFA were determined as described previously (220). For $\mathrm{NH}_{3}$ determination, $750 \mu \mathrm{L}$ reaction fluid was deproteinised by $750 \mu \mathrm{L}$ trichloroacetate (100 g per litre of trichloroacetate neutralised by $\mathrm{NaOH}$ ). Samples were centrifuged at $14000 \times \mathrm{g}$ at $20{ }^{\circ} \mathrm{C}$ for $10 \mathrm{~min}$. Concentration of $\mathrm{NH}_{3}$ in the supernatant was determined using a Skalar Autoanalyzer SA1050 (Skalar Analytical BV, Breda, the Netherlands). For SCFA analysis, $750 \mu \mathrm{L}$ reaction fluid was acidified with $750 \mu \mathrm{L}$ internal standard (isocaproic acid) in a $40 \mathrm{~g} \mathrm{~L}^{-1} \mathrm{O}$-phosphoric acid solution. SCFAs were analysed using a gas chromatograph (Fisons HRGCMega 2, CE Instruments, Milan, Italy) with a split/splitless injector operated in split mode (split ratio 1: 9) and fitted to a flame ionisation detector, using a capillary column (30m, internal diameter $0.53 \mathrm{~mm}$, film thickness $1.0 \mu \mathrm{m}$; Agilent J\&W HP-FFAP, Santa Clara, CA, USA) with hydrogen as the carrier gas ( $25 \mathrm{kPa}$ pressure) and isocaproic acid used as an internal standard. The starting temperature of the column was set at $80^{\circ} \mathrm{C}$ for $1 \mathrm{~min}$, followed by a $20^{\circ} \mathrm{C} \mathrm{min}-1$ increase to $120{ }^{\circ} \mathrm{C}$, followed by a $6.1{ }^{\circ} \mathrm{C} \mathrm{min} \mathrm{m}^{-1}$ increase to $205{ }^{\circ} \mathrm{C}$, where temperature was maintained for $2 \mathrm{~min}$. Lactate was measured using the Lactate Colorimetric Assay Kit II (K627, Biovision) according to the manufacturer's instructions, including the optional filtration step with 
Amicon 10K spin columns (Z677108-96EA, Sigma Aldrich, centrifugation for 20 minutes at $21.100 \times \mathrm{g}$ ). Briefly, samples were tested in four different dilutions; undiluted, 31/3 times, 10 times and 50 times diluted in "Lactate Assay Buffer" and $50 \mu \mathrm{L}$ sample or dilution was transferred to a 96-wells plate. Subsequently, $50 \mu \mathrm{L}$ reaction mix composed of Lactate Substrate Mix $(2 \mu \mathrm{L})$, Lactate Enzyme Mix (2 $\mu \mathrm{L})$, and Lactate Assay Buffer $(46 \mu \mathrm{L})$, was added to each sample and incubated for 30 minutes at room temperature. Optical density was measured at $450 \mathrm{~nm}$ and concentrations of extracellular lactate were calculated based on a lactate calibration curve supplied in the kit.

\section{Oral gavage and tissue sampling}

Carp of 9 months (20-40 g, $\mathrm{n}=20$ per group) were starved overnight and anaesthetized in $0.15 \mathrm{~g} / \mathrm{L}$ Tricaine Methane Sulfonate (TMS) (Crescent Research Chemicals, Phoenix, USA) in aquarium water buffered with $0.3 \mathrm{~g} / \mathrm{L}$ sodium bicarbonate. Anaesthetized fish received an oral gavage with $100 \mu \mathrm{L}$ of PBS (Lonza, LO BE17-516F) or MacroGard ${ }^{\circledR}$ dissolved in PBS (10 mg/mL, $1 \mathrm{mg} / \mathrm{mL}$ per fish) using a $200 \mu \mathrm{L}$ pipet. At 7 and 14 days post-gavage, fish were anaesthetized in 0.3 $\mathrm{g} / \mathrm{L}$ Tricaine Methane Sulfonate (TMS) (Crescent Research Chemicals, Phoenix, USA) in aquarium water buffered with $0.6 \mathrm{~g} / \mathrm{L}$ sodium bicarbonate and bled via the caudal vein. The third segment of the intestine was isolated and intestinal content of the third segment was collected by scraping, snap-frozen in liquid nitrogen and stored at $-80^{\circ} \mathrm{C}$. A tissue sample of the third segment of the intestine was snapfrozen in liquid nitrogen and stored at $-80^{\circ} \mathrm{C}$.

\section{Total RNA isolation from intestinal samples}

Total RNA from isolated tissue samples was isolated using the RNeasy mini Kit (Qiagen, 74106), including on-column DNase treatment, according to the manufacturer's instructions, and stored at $-80^{\circ} \mathrm{C}$. Prior to cDNA synthesis, 1000 ng total RNA was treated with DNase I, Amplification Grade (Invitrogen), and cDNA was synthesized using random primers (300 ng) and Superscript III First-Strand Synthesis for RT-PCR (Invitrogen). CDNA samples were diluted in nuclease-free water prior to real-time quantitative PCR (RT-qPCR) analysis.

\section{Gene expression analysis}

Gene expression was measured with RT-qPCR using ABsolute qPCR SYBR Green Mix (Thermo Scientific) in a Rotor-Gene 6000 (Corbett Research), and fluorescence data were analysed using Rotor-Gene Analysis software version 1.7 . The relative expression ratio $(R)$ of each sample was calculated according to the Pfaffl method (173) based on the take-off deviation of sample versus each of the PBS controls and normalized relative to the $\mathrm{s} 11$ protein of the 40 s subunit as reference gene (see Table 1). 
Table 1. Overview of RT-qPCR primers used for in the current study. cypCar numbers identify ORFs in the draft carp genome (bioproject: PRJNA73579) that were also confirmed by RNA sequencing, LHQP number refers to the accession number of the associated scaffold.

\begin{tabular}{|c|c|c|c|}
\hline Primer & Forward $\left(5^{\prime}-3^{\prime}\right)$ & Reverse $\left(5^{\prime}-3^{\prime}\right)$ & $\begin{array}{l}\text { GenBank } \\
\text { Accession No. }\end{array}$ \\
\hline $40 \mathrm{~s}$ & CCGTGGGTGACATCGTTACA & $\begin{array}{l}\text { TCAGGACATTGAACCTCACTGT } \\
\text { CT }\end{array}$ & AB012087 \\
\hline$c x c a$ & GGGTGTAGATCCACGCTGTC & CTTTACAGTGTGGGCTTGGAG & AJ550164 \\
\hline$c x c b$ & GCTGCCTGCTTGTTGTAGAG & ATCTGTTTTGGAGGAACCA & AB082985 \\
\hline$i l 1 b$ & AAGGAGGCCAGTGGCTCTGT & $\begin{array}{l}\text { CCTGAAGAAGAGGAGGAGGCT } \\
\text { GTCA }\end{array}$ & AJ245635 \\
\hline$i / 6 a$ & CAGATAGCGGACGGAGGGGC & GCGGGTCTCTTCGTGTCTT & KC858890 \\
\hline$i / 6 b$ & GGCGTATGAAGGAGCGAAGA & ATCTGACCGATAGAGGAGCG & KC858889 \\
\hline tnfaa1 & $\begin{array}{l}\text { GAGCTTCACGAGGACTAATAG } \\
\text { ACAGT }\end{array}$ & CTGCGGTAAGGGCAGCAATC & AJ311800 \\
\hline tnfaa2 & CGGCACGAGGAGAAACCGAGC & $\begin{array}{l}\text { CATCGTTGTGTCTGTTAGTAAG } \\
\text { TTC }\end{array}$ & AJ311801 \\
\hline tnfab1 & $\begin{array}{l}\text { GAAGACGATGAAGATGATACC } \\
\text { AT }\end{array}$ & AAGTGGTTTTCTCATCCTCAA & $\begin{array}{l}\text { cypCar_00029601, } \\
\text { LHQP01065580 }\end{array}$ \\
\hline tnfab2 & $\begin{array}{l}\text { CTTGGACGAAGCCGATGAAGA } \\
\text { C }\end{array}$ & ATCTTGTGACTGGCAAACA & $\begin{array}{l}\text { cypCar_00023012, } \\
\text { LHQP01037150 }\end{array}$ \\
\hline il10a & CGCCAGCATAAAGAACTCA & TGCCAAATACTGCTCAATGT & $\begin{array}{l}\text { cypCar_00007086, } \\
\text { LHQP01030085 }\end{array}$ \\
\hline$i / 10 b$ & CGCCAGCATAAAGAACTCGT & TGCCAAATACTGCTCGATGT & $\begin{array}{l}\text { cypCar_00012555, } \\
\text { LHQP01021640 }\end{array}$ \\
\hline $\operatorname{tr} 3.1$ & GTTATCCCTGGCGCATAATA & $\begin{array}{l}\text { TCTTCAATAATTGGTAAGGATG } \\
\text { ATG }\end{array}$ & KF387571 \\
\hline t/r3.2 & GTTTATCCCTGGAGCATAACT & $\begin{array}{l}\text { CTTCAATAACTGGTAAAGACGA } \\
\text { AC }\end{array}$ & KF387572 \\
\hline$m \times 1$ & ACAATTTGCGGTCTTTGAGA & CCCTGCCATTTCTCTTCG & $\begin{array}{l}\text { cypCar_00015892, } \\
\text { LHQP01004675 }\end{array}$ \\
\hline$m \times 2$ & GCTTACGGTCTCTGGGG & $\begin{array}{l}\text { TGGTTTCATCTTTAGTTCTTATC } \\
\text { ATC }\end{array}$ & $\begin{array}{l}\text { cypCar_00029512, } \\
\text { LHQP01026950 }\end{array}$ \\
\hline$m \times 3$ & ACAAAGGACAATAACTGGCG & GAGGTCAGGAACATCACTG & $\begin{array}{l}\text { cypCar_00017679, } \\
\text { LHQP01012215 }\end{array}$ \\
\hline$m \times 4$ & CTAGAGTTGCCACTGCC & TCCAGTTGAATCCACTTCG & $\begin{array}{l}\text { cypCar_00025664, } \\
\text { LHQP01010684 }\end{array}$ \\
\hline$m \times 5$ & ACTGAAGTGTGTGTTTITGG & CAGACCTGGTAGATAAAGGAG & $\begin{array}{l}\text { cypCar_00012158, } \\
\text { LHQP01006771 }\end{array}$ \\
\hline stat1 & GAGACGGAGGAATCACC & GGATGTCTGGGTAAAGGTAG & $\begin{array}{l}\text { cypCar_00011644, } \\
\text { LHQP01012446 and } \\
\text { cypCar_00037712, } \\
\text { LHQP01035256.1 }\end{array}$ \\
\hline
\end{tabular}




\section{Results}

\section{Characterization of intestinal microbial composition implies $\boldsymbol{\beta}$-glucan fermentation capacity}

Analysis of $16 \mathrm{~S}$ ribosomal RNA rather than DNA provided insight into the active intestinal microbial communities (221). Furthermore, identification of bacterial microbiota up to family level allowed us to investigate the presence of bacteria with theoretical fermenting capacities. Intestinal content of the third segment of the intestine from $n=5$ individual fish was collected for 16S rRNA sequencing, revealing a total of $n=55$ active operational taxonomic units (OTUs) in the intestinal content of unhandled carp.

By far, the most represented Phyla in the carp intestinal microbiota were Fusobacteriaceae ( $71 \%$ of total reads), whereas Bacteroidaceae $(21 \%)$, Proteobacteria (5\%) and Firmicutes (2\%) were considerably less frequent (Figure $1 \mathrm{~A})$. The majority of Proteobacteria belonged to the order of Gammaproteobacteria $(95 \%)$, while the rest belonged to the Betaproteobacteria $(4 \%)$. Looking at the distribution per Family in the active microbiota, the most abundant family were Fusobacteriaceae (71\%), followed by Bacteroidaceae (16\%), Porphyromonadaceae (5\%), Vibrionaceae (3\%), Aeromonadaceae $(1.5 \%)$ and Erysipelotrichaceae (1.4\%) (Figure 1B). Finally, looking at putative genus level it becomes clear that the majority of Fusobacteriaceae are member of the Cetobacterium genus, as $71 \%$ of the total reads belong to this genus, whereas all

A

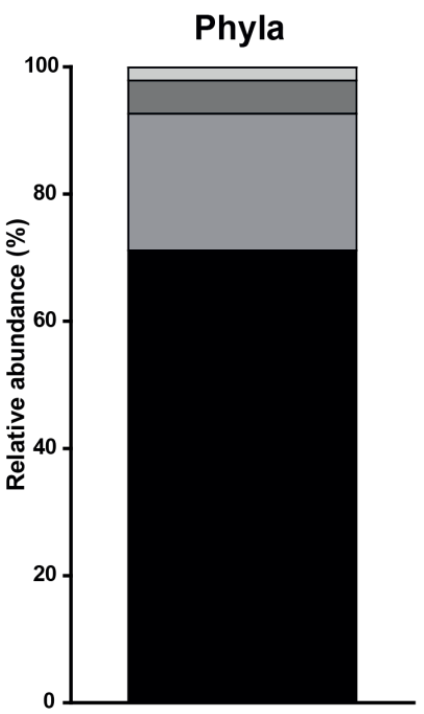

B

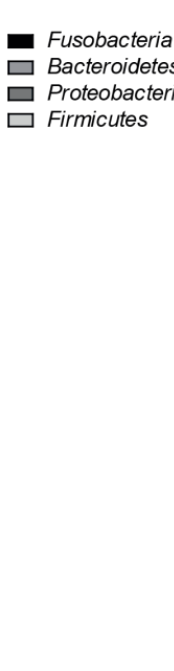

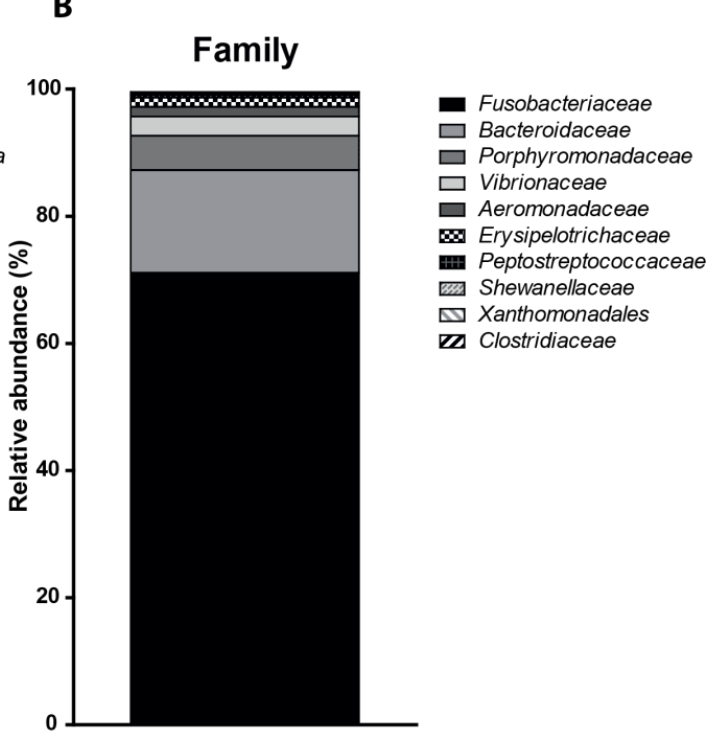

Figure 1. Relative abundance of active bacteria in the intestinal microbiota of common carp. Characterization with 16S rRNA sequencing of the intestinal microbiota of unhandled common carp. A. Relative abundance of phyla. B. Relative abundance of family. Xanthomonadales refers to family Xanthomonadales Incertae sedis. 
further reads belong to a single OTU. The most active genera are Bacteroides (16\%, 2 OTUs), Vibrio (3\%), Aeromonas (1.5\%) and two different uncultured genera of the families Porphyromonadaceae $(4.5 \%, 3$ OTUs) and Erysipelotrichaceae (1.1\%, 1 OTU). Although the sequencing effort could not identify bacteria at species level, abundance of the Bacteroides genus well known to express $\beta$-glucan degrading genes (70), could imply that carp microbiota is able to ferment $\beta$-glucans.

\section{In vitro batch analysis confirms fermentation of $\beta$-glucans}

In order to investigate the $\beta$-glucan fermentation ability of the normal intestinal microbiota of carp, in vitro fermentation was performed (Figure 2A). Through an in vitro batch culture system, fermentation of different substrates could be analysed. As a measure for fermentation, cumulative gas production was measured over time. As a negative control, minor cumulative gas production was

A

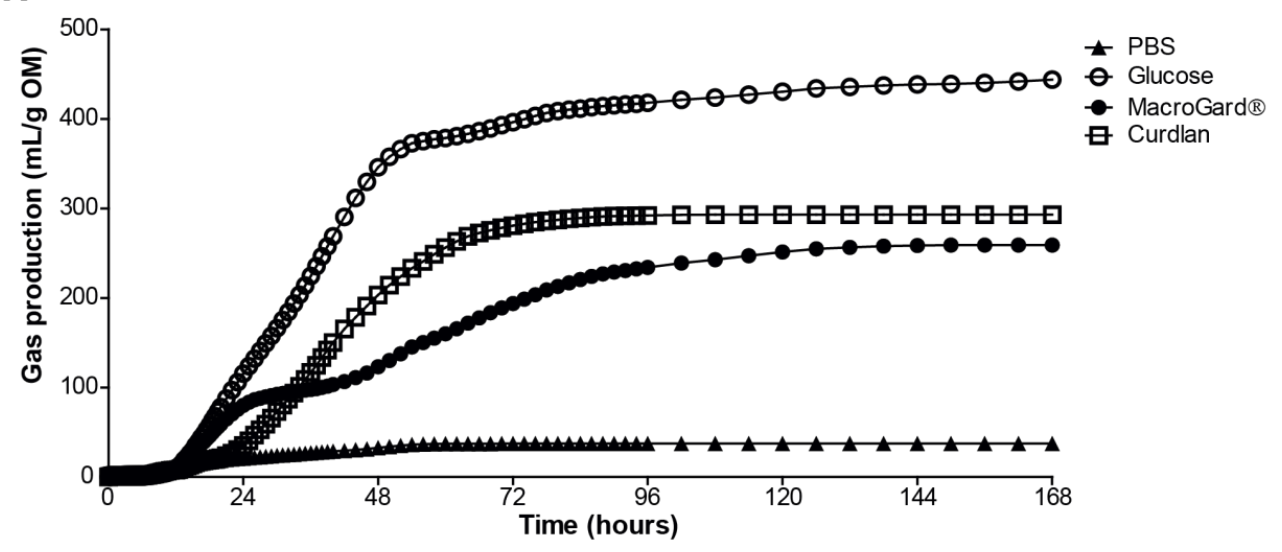

B

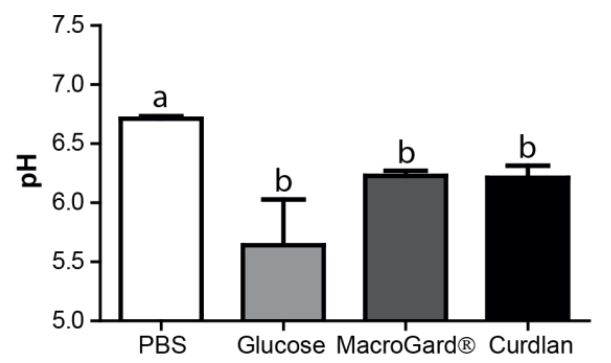

C

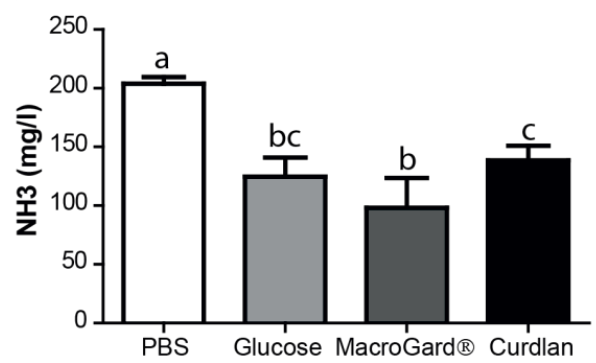

Figure 2. Carp normal microbiota is capable of in vitro fermentation of different $\boldsymbol{\beta}$-glucans. A. Cumulative gas production over $168 \mathrm{~h}$ as a result of in vitro fermentation of PBS, glucose, MacroGard ${ }^{\circledR}$ and curdlan by carp normal intestinal microbiota. Data shown as mean of $\mathrm{n}=5$ independent intestinal pools. B. $\mathrm{pH}$ of fermentation fluids after $168 \mathrm{~h}$ of in vitro fermentation (mean $+S D, n=5$ ). Significant differences between groups were assessed by Welch's ANOVA followed by Games-Howell test. C. Ammonia $\left(\mathrm{NH}_{3}\right)$ accumulation in fermentation fluids after $168 \mathrm{~h}$ of in vitro fermentation (mean $+\mathrm{SD}, \mathrm{n}=$ 5). Significant differences between groups were assessed by one-way ANOVA followed by Tukey test. Groups with different letters are statistically different from one another. 
observed in the PBS group, similar to gas production in the negative control without substrate (data not shown). As a positive control, fermentation of the readily digestible mono-saccharide glucose was observed, resulting in the highest cumulative gas production. Two different $\beta$-glucan preparations were studied; curdlan, a high molecular weight linear polymer consisting of $\beta$-1-3-linked glucose residues from Alcaligenes faecalis and MacroGard ${ }^{\circledR}$, a branched polymer $\beta$ $(1,3 / 1,6)$-glucose feed additive. In comparison, fermentation of curdlan resulted in the most continuous and highest cumulative gas production, with fermentation of MacroGard ${ }^{\circledR}$ showing an intermediate plateau around $24-36$ hours. No matter the different dynamics of gas production between curdlan and MacroGard ${ }^{\circledR}$, carp normal intestinal microbes were able to ferment $\beta$-glucans.

Analysis of fermentation liquid following in vitro fermentation revealed differences in $\mathrm{pH}$ and $\mathrm{NH}_{3}$ between substrates. At initialization of in vitro fermentation, $\mathrm{pH}$ per substrate and per inoculate did not differ (data not shown). In comparison, a (nonsignificant) trend towards lower $\mathrm{pH}$ was observed in the glucose group, compared to the two $\beta$-glucan treated groups. No difference in $\mathrm{pH}$ was observed between MacroGard ${ }^{\circledR}$ and curdlan treatment (Figure 2B). Ammonia production was significantly higher in the PBS group compared to all other groups (Figure 2C). Interestingly, $\mathrm{NH}_{3}$ production was different between curdlan and MacroGard ${ }^{\circledR}$, providing further evidence for differences in fermentation dynamics between the two $\beta$-glucans.

\section{In vitro fermentation of $\beta$-glucans results in significantly increased SCFA levels}

From the in vitro batch culture analysis it became evident that the intestinal microbiota of carp can ferment $\beta$-glucans up to a certain degree. Metabolites produced by the microbiota during fermentation such as SCFA are necessary components for immune homeostasis (222). Accumulation of SCFA metabolites produced during $\beta$-glucan fermentation was analysed in the in vitro fermentation liquid. Significant differences in SCFA profiles between the readily-fermentable substrate glucose and the two independent $\beta$-glucan preparations could be observed (Figure 3). Overall, a clear production of acetate, butyrate and propionate was observed after fermenting either curdlan or MacroGard ${ }^{\circledR}$ in vitro. Comparing the two different $\beta$-glucan preparations showed no differences in acetate production but revealed an interesting inverted trend for butyrate and propionate levels. After fermentation of curdlan, higher butyrate concentrations were measured, while after fermentation of MacroGard ${ }^{\circledR}$ higher propionate concentrations were measured (Figure 3B-C). In both $\beta$-glucan groups total SCFA levels were higher than in the glucose group, however, no differences were observed between both $\beta$-glucan groups (Figure 3E). Overall, differential production of SCFAs could be observed in both $\beta$-glucan groups, with fermentation of MacroGard ${ }^{\circledR}$ specifically resulting in an increase in propionate. 
A

Acetate

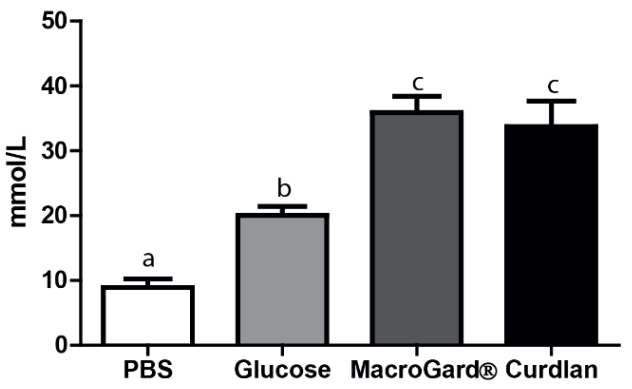

C

Propionate

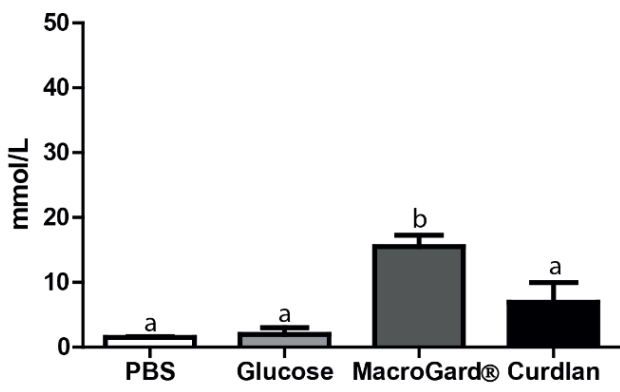

$\mathbf{E}$

Total SCFA

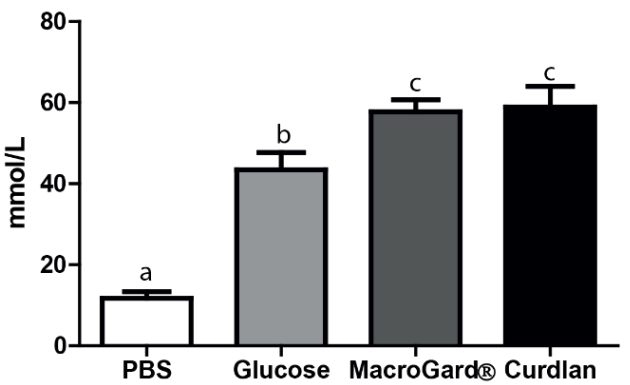

B

Butyrate

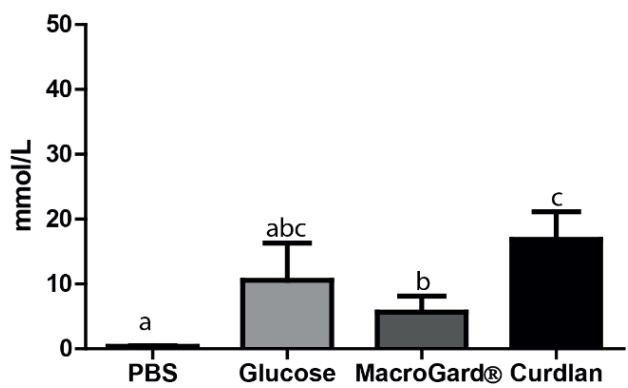

D

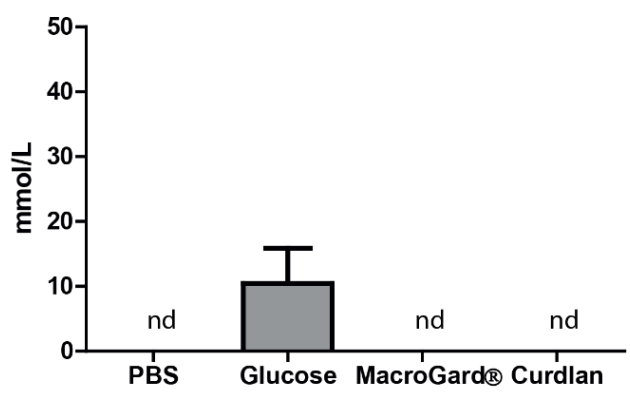

Figure 3. Differential SCFA profiles after in vitro fermentation of $\boldsymbol{\beta}$-glucan. SCFA levels in the fermentation liquid following $168 \mathrm{~h}$ in vitro fermentation of PBS, glucose, MacroGard ${ }^{\circledR}$ or curdlan. A-E. Acetate, butyrate, propionate and lactate, or total sum. Total SCFA levels were calculated by adding up all analysed SCFA levels including branched chain short chain fatty acids. Bars indicate mean $+S D$ of $n=5$ independent intestinal pools. Significant differences between groups were assessed by one-way ANOVA followed by Tukey test (4A, D and E) or by Welch's ANOVA followed by Games-Howell test (4B and C). Groups with different letters are statistically different from one another. 


\section{Oral gavage with $\beta$-glucans can alter microbiota composition of carp}

In vitro fermentation of $\beta$-glucans by intestinal microbes of carp revealed both the capacity to ferment $\beta$-glucans as well as an accumulation of propionate after fermentation of MacroGard ${ }^{\circledR}$. We progressed to investigate the effects of in vivo $\beta$ glucan supplementation on the intestinal microbiota, 7 and 14 days after a single oral gavage treatment with PBS or MacroGard ${ }^{\circledR}$. Overall, analysis of diversity indicated a trend towards a higher number of OTUs upon either PBS or $\beta$-glucan treatment compared to the unhandled group (Fig. 4A), however, only PBS-treated control fish on day 7 showed a significantly higher number of OTUs compared to those in the unhandled group $(p<0.05)$ (Fig 4A, Supplementary table 1$)$. The alpha diversity matrices, a measure for the mean species diversity in a single group, showed no significant differences between unhandled, control and treatment groups (Supplementary table 1).

Taking into account the OTU abundance, both control (PBS) and MacroGard ${ }^{\circledR}$ treated groups were separated based on a principle component analysis (Figure 4B). To further investigate the differences in abundance between these two treatment groups two different statistical analyses were used, cumulative sum scaling (CSS) (Figure 4C-D; Supplementary table 2) and random forest analysis followed by the Boruta feature selection (Supplementary figure 1, Supplementary table 3). Specifically looking at increased OTUs in the treatment groups, identified by both CSS and the random forest analysis, revealed increased abundance of members belonging to the families Rhodocyclaceae and Streptococcaceae on day 7 post-treatment with MacroGard ${ }^{\circledR}$. On day 14 post-treatment, there was only a single OTU increased by the treatment, belonged to the Rhodocyclaceae. Although the single oral gavage as a treatment was subtle, the abundance of several OTUs were shifted due to the treatment with $\beta$-glucans. 
A

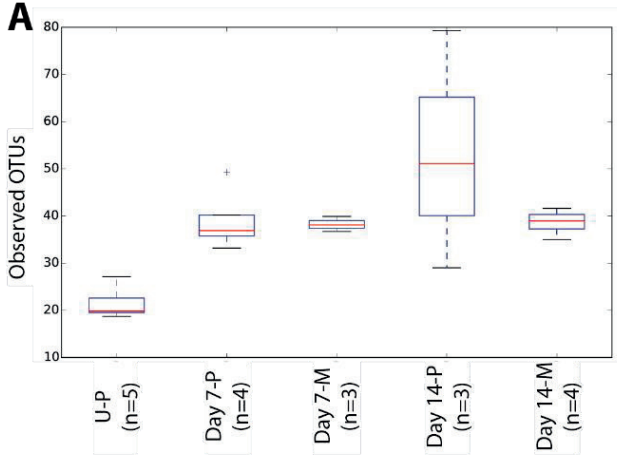

C

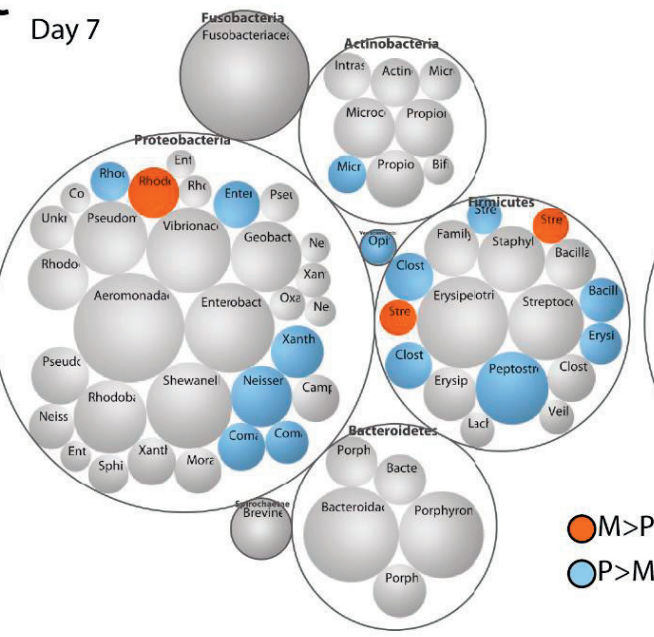

B

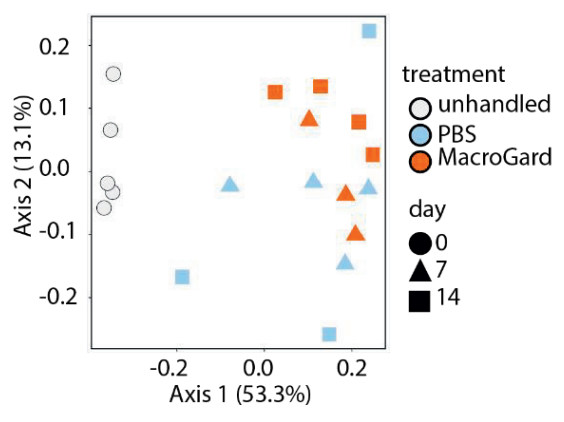

D
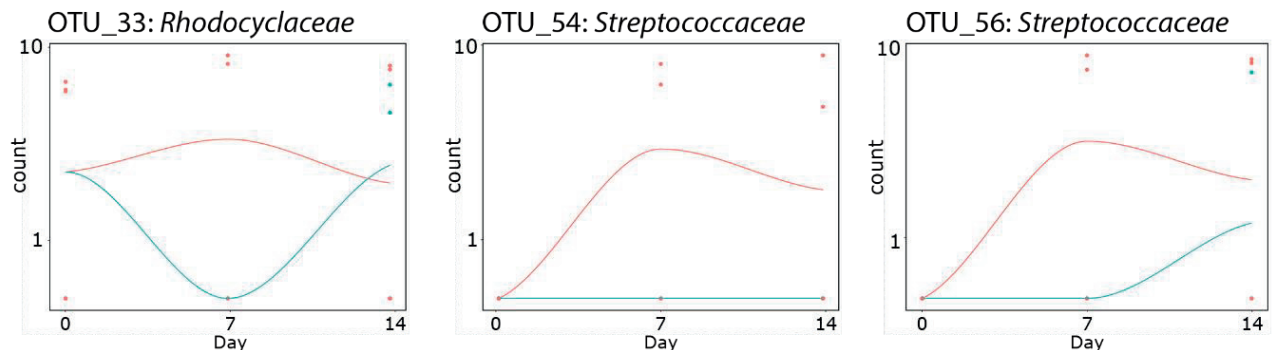

Figure 4. Oral gavage with $\beta$-glucans induces a shift in intestinal microbial community of carp. A. Number of observed operational taxonomic units (OTUs; alpha diversity) of the intestinal microbiota showing a higher diversity after treatment with PBS (P) and MacroGard ${ }^{\circledR}(\mathrm{M})$ compared to unhandled (U) fish. B. Principal component analysis of microbiota showing a shift in community upon $\beta$-glucan treatment. C. Differences based on cumulative sum scaling and fitZIG contrasts showing significantly more abundant OTUs between PBS- and MacroGard ${ }^{\circledR}$-treated carp at day 7 and day 14 after gavage. The size of each circle indicates the abundance of the respective family overall in all samples. D. Three OTUs were significantly more abundant in MacroGard ${ }^{\circledR}$-treated carp at day 7 showing different dynamics in time. 


\section{In vivo treatment with $\beta$-glucans modulates expression of immune genes}

To follow up on the above-observed shift in carp intestinal microbiota in vivo, simultaneous modulation of local immune gene expression was analysed. Therefore, we analysed the gene expression from intestinal tissue samples collected at the exact same location at which the intestinal content was collected for $16 \mathrm{~S}$ rRNA sequencing. Based on an earlier observation following feeding with MacroGard $^{\circledR}(111)$, expression of several cytokines was examined. We found significant down-regulation of $c x c a, i / 1 b, i / 6 a$ and tnfaa2 at 7 days (Figure 6A) and sometimes 14 days (Figure 6B) post-treatment with MacroGard ${ }^{\circledR}$. In addition, based on an original hypothesis of sensing dsRNA from lactate bacteria (76), an increase of which could not readily be confirmed by the $\beta$-glucan treatment-

A

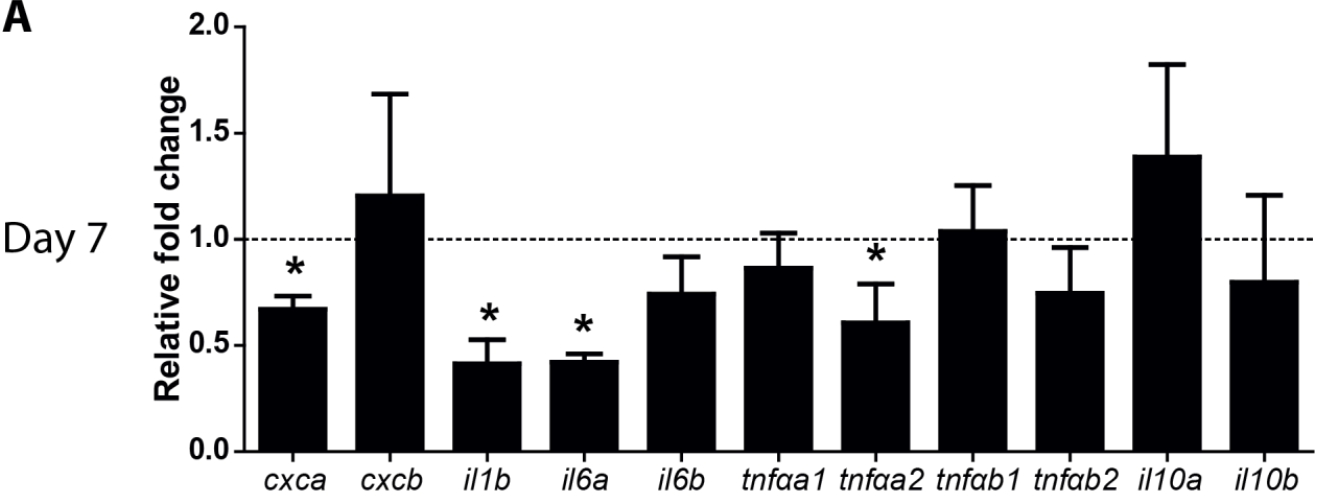

B

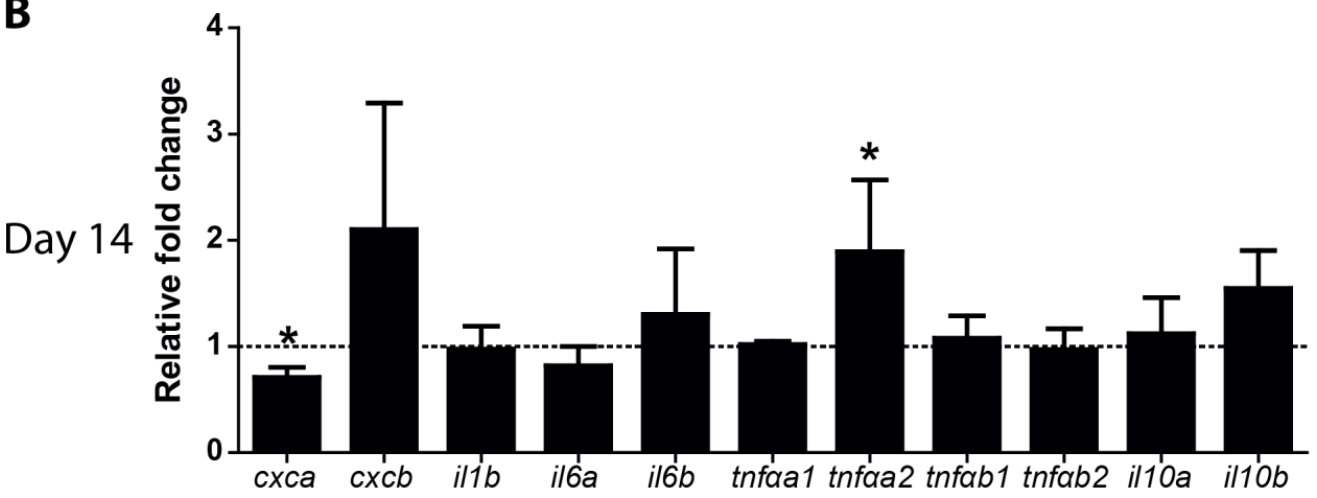

Figure 5. Oral gavage with $\boldsymbol{\beta}$-glucans alters cytokine gene expression in the third segment of carp intestine. Gene expression at day 7 (A) and day 14 (B) in the third gut segment of the treated group compared to control group (dotted line), as measured by RT-qPCR. A. Significantly lower expression of $c x c a, i / 1 b$, il6a and tnfaa2 can be observed. A trend towards lower expression of il6b $(p=0.062)$ and tnfab2 $(p=0.090)$ could be observed in the treated group. B. Significantly lower expression of $c x c a$ and a significant increase in tnfaa 2 expression could be observed in the treated group. Asterisk $(*)$ indicates significant difference between expression between the group orally gavaged with PBS and the group orally gavaged with MacroGard ${ }^{\circledR}$ as assessed by one-way ANOVA. 
induced shift, measurement of relevant gene expression was also included. We found significant down-regulation of t/r3.1 and $m \times 3$ at 7 days (Supplementary figure $2 \mathrm{~A}$ ) and up-regulation of $m \times 4$ at 14 days (supplementary Figure 2B). Taken together, we observed several immunomodulatory effects on gene expression following in vivo treatment with MacroGard ${ }^{\circledR}$, coinciding with a shift in intestinal microbial composition.

\section{Discussion}

To investigate whether $\beta$-glucans can alter composition or activity of the intestinal microbiota we first characterized the active intestinal microbiota of unhandled carp. We observed, among others, a prominent presence of the genus Bacteroides, which are well-known for their ability to degrade carbohydrates. The mere presence of the Bacteroides genus is interesting because some species of the Bacteroides genus have been shown able to specifically degrade $\beta$-glucans (70). Subsequently, we confirmed a theoretical $\beta$-glucan fermenting ability of carp microbiota by in vitro batch culture system, in which we used intestinal microbiota of carp to test fermentation of two different $\beta$-glucan preparations. As a result of this in vitro fermentation, we observed a production of several SCFAs, of which propionate could be specifically linked to the fermentation of MacroGard ${ }^{\circledR}$. To follow up, we investigated the effects of a single oral gavage with MacroGard ${ }^{\circledR}$ on the intestinal microbiota and on local immune gene expression. We observed an increase in abundance at seven days post treatment with $\beta$-glucans of three OTUs, one belonging to the family Rhodocyclaceae and the other two belonging to Streptococcaceae. Coinciding with this increase, we also observed a clear reduction in IFN related and cytokine gene expression.

We identified four abundant phyla in our carp normal intestinal microbiota (Fusobacteria, Bacteroidetes, Gammaproteobacteria, Firmicutes) using 16S rRNA analysis. A comparable division of phyla in carp normal intestinal microbiota was also found previously using $16 \mathrm{~S}$ rRNA pyrosequencing (223), suggesting that indeed this could be considered the core intestinal microbiota of common carp. In fact, despite possible fish species-specific differences with respect to relative abundance (223-226), the same four phyla appear common to intestines of fishes. Of the phylum Fusobacteria, the genus Cetobacterium clearly dominated abundance and appears to do so in omnivorous and carnivorous more than in herbivorous fishes (225). The genus Bacteroides appeared as the second most abundant microbial species and is particularly interesting with respect to fermentation of carbohydrates.

The genus Bacteroides has been linked to carbohydrate degradation and is capable of degrading even highly complex carbohydrates $(69,227,228)$. Degradation of carbohydrates is achieved with so-called polysaccharide utilization loci (PUL) of which there can be many different ones, each related to the degradation of a specific glycan or group of related glycans (229). Members of the Bacteroides found in the gastro-intestinal tract of humans have been shown able to degrade 
several different $\beta$-glucans, among which barley-derived $\beta$-1,4-glucans (69) and fungus- derived $\beta-1,6$-glucans (70). The presence of the Bacteroides genus in the normal intestinal microbiota might suggest that carp should be capable of degrading $\beta$-glucans. We thus performed an in vitro batch culture experiment with two different $\beta$-glucans as substrates; MacroGard ${ }^{\circledR}$, a branched $\beta-1,3 / 1,6$-glucan isolated from the yeast Saccharomyces cerevisiae and commonly used in aquaculture practices, and curdlan, a linear $\beta-1,3$-glucan isolated from the bacteria Alcaligenes faecalis. Each showed distinct but slightly different fermentation curves, possibly in part related to 'contamination' of MacroGard ${ }^{\circledR}$ with products such as chitin and mannose (46), of which the latter could inhibit carbohydrate uptake of bacteria under specific conditions (230). Fermentation also led to higher butyrate production associated with curdlan and higher propionate production associated with MacroGard ${ }^{\circledR}$. Again, the difference might be related in part to the presence of products such as chitin because, at least in tilapia, intestinal microbiota were able to ferment chitin resulting in acetate and propionate but no butyrate (193). Regardless of the above, in vitro fermentation of $\beta$-glucans by the intestinal microbiota of carp resulted in increased gas and SCFA production.

Several studies in mice have connected oral intake of fibres to increased SCFA levels, both local and systemic. Moreover, these studies showed connections between increased SCFA levels and reductions in pathogenesis. For example, mice fed with oat bran derived $\beta$-glucan enriched diets had higher SCFA levels in the caecum, especially after having been fed diets with practically insoluble $\beta$-glucan of the heavy molecular weight (74). Mice fed with high fibre diets showed systemic increases in propionate levels, while oral supplementation with propionate reduced allergy induced inflammation in the lungs (231). Earlier studies in fish have hinted at the ability of fish microbiota to degrade carbohydrates, observing fermentation of several carbohydrates and production of SCFAs by intestinal microbes of tilapia, European sea bass or common carp (192-194) or showing growth of monocultures of fish intestinal bacteria on different carbohydrate sources, including $\beta$-glucan (191). While the connections between differential SCFA levels due to diet and reduced pathogenesis have not been made in fish, here we show that the intestinal microbiota of fish can play a role in the fermentation of carbohydrates.

Studies in mammals have suggested connections between intake of high fibre diets and reduced systemic inflammation could be mediated by the increased expression of receptors for SCFAs such as the G-protein coupled receptors GPR41 and GPR43 (also known as free fatty acid receptor 2 or FFAR2) (232). SCFAs activities can be both anti- and pro-inflammatory and would be mediated by GPR41, GPR43, to lesser extent GPR109A, or via their effect on histone deacetylases (HDACs) (as reviewed by (72)). At present, these free fatty acid receptors have not been described for fish, but it would be of great interest to study expression of free fatty acid receptors in the context of our findings.

We observed changes in the SCFA profile after in vitro fermentation and observed an increase in Rhodocyclaceae and Streptococcaceae genera in vivo. Although the 
change in SCFA profile associated with fermentation of $\beta$-glucans might explain the observed modulation of immune gene expression in the intestine, there might be other mechanisms at play that could modulate the immune response in the intestine. In mice, commensal lactate bacteria can induce an antiviral IFN response via shedding of double stranded RNA which, recognized by intestinal dendritic cells (DCs) via a TIr3 mediated mechanism, can lead to the production of IFN $\beta$, which in turn dampens the expression of IL6 and TNFa (76). In fish, several studies observed effects of $\beta$-glucan supplementation on the presence of lactate bacteria $(78,79)$, although this shift did not stand out in our present study, at seven days post treatment there was a significant increase in a OTU belonging to the Streptococcus genus, belonging to the order of Lactobacillales (lactate bacteria). In a previous study we noticed a concurrent increase in $m x$ and $t / r 3$ expression in the intestine of carp fed with $\beta$-glucans (111). Another study also observed prolonged $m x$ expression following $\beta$-glucan supplementation (233). In our present study we again noticed in carp intestine modulation of expression of anti-viral genes such as $m x$, suggesting immunomodulation by the increased presence of dsRNA should not be excluded.

No matter the presence in the fish intestine of free fatty acids or dsRNA and their receptors, or not, immunomodulation was evident. In a previous study we reported on the inhibition of expression of several pro-inflammatory genes in the intestine of carp following 25 days of dietary supplementation with $\beta$-glucans. Expression of $i / 1 \beta$, tnfa and il10 in the intestine was strongly inhibited in this in vivo feeding experiment (111), an observation that was partly confirmed by our in vivo single gavage study. In mammals, propionate and butyrate are known to regulate inflammatory responses in epithelial cells and leukocytes (232, 234-237). It remains to be determined whether the inhibition of expression of pro-inflammatory genes in carp intestine was modulated by SCFAs or by bacterial substances.

In the end, the observed effects of $\beta$-glucans on the microbiota and on the intestinal gene expression cannot be definitively mechanisms, either direct effects of the $\beta$-glucans on the intestine or indirect effects through prebiotic effects on the intestinal microbiota. The coinciding change in microbiota and immuno-regulation on day 7 post-treatment at the same location in the intestine, could argue for prebiotic effects from $\beta$-glucans on the intestinal microbiota resulting in immunomodulation in the host via SCFAs. At the same time, a regulation of t/r3 and $m x$ paralogs was also observed following $\beta$-glucan supplementation, coinciding with an increase in a Lactobacillales genus on day 7 post-treatment. Therefore, we cannot rule out that the intestinal immuno-modulation is a result of the production of other molecules by the microbiota, such as double stranded RNA. It can be of interest to analyse the effects of $\beta$-glucans on sterile intestinal samples, for example with ex vivo stimulation of gut tissue, to investigate whether $\beta$-glucans directly affect the gene expression in the intestine resulting in regulation of several immune genes or whether the microbiota plays a crucial part in this effect. Such an experimental setup would allow for selectively testing the effects of SCFAs and other microbiota produced molecules. Although not conclusive, the present study 
has provided insights into possible indirect mechanisms of $\beta$-glucan immunomodulation and paves the road for future studies to unravel a definitive mechanism of action through which $\beta$-glucan supplementation can modulate immune responses.

\section{Acknowledgements}

Adrià López Nadal and Mark Goldman from the Cell Biology and Immunology group and Saskia van Laar from the Animal Nutrition group are gratefully acknowledged for their help during the in vitro batch culture experiment. Johan Schrama from the Aquaculture and Fisheries group is gratefully acknowledged for his suggestions and helpful advice on SCFAs. Research leading to this review was funded by the Netherlands Organisation for Scientific Research and São Paulo Research Foundation, Brazil (FAPESP) as part of the Joint Research Projects BioBased Economy NWO-FAPESP Programme (Project number 729.004.002). 


\section{Supplementary material}

Supplementary table 1 . Comparison of alpha diversity matrices of operational taxonomic units (OTUs) detected in guts of unhandled carp (UO) and after 7 or 14 days of gavage with PBS (P) and MacroGard (M) treated carp. Significant differences were determined on rarefied data using two-sample t-test will be nonparametric using 999 Monte Carlo permutations ${ }^{1}$.

\begin{tabular}{|c|c|c|c|c|c|c|c|}
\hline Group1 & Group2 & $\begin{array}{c}\text { Group1 } \\
\text { mean }\end{array}$ & $\begin{array}{c}\text { Group1 } \\
\text { std }\end{array}$ & $\begin{array}{c}\text { Group2 } \\
\text { mean }\end{array}$ & $\begin{array}{c}\text { Group2 } \\
\text { std }\end{array}$ & t stat & p-value \\
\hline \multicolumn{8}{|c|}{ Observed_OTUs } \\
\hline B_P7 & A_UO & 39.05 & 6.06 & 21.54 & 3.08 & 4.96 & 0.03 \\
\hline C_M7 & A_U0 & 38.23 & 1.31 & 21.54 & 3.08 & 7.73 & 0.07 \\
\hline D_P14 & A_U0 & 53.13 & 20.59 & 21.54 & 3.08 & 2.92 & 0.15 \\
\hline E_M14 & A_U0 & 38.63 & 2.45 & 21.54 & 3.08 & 7.98 & 0.09 \\
\hline B_P7 & C_M7 & 39.05 & 6.06 & 38.23 & 1.31 & 0.19 & 1.00 \\
\hline D_P14 & C_M7 & 53.13 & 20.59 & 38.23 & 1.31 & 1.02 & 1.00 \\
\hline E_M14 & C_M7 & 38.63 & 2.45 & 38.23 & 1.31 & 0.21 & 1.00 \\
\hline B_P7 & D_P14 & 39.05 & 6.06 & 53.13 & 20.59 & -1.09 & 1.00 \\
\hline B_P7 & E_M14 & 39.05 & 6.06 & 38.63 & 2.45 & 0.11 & 1.00 \\
\hline D_P14 & E_M14 & 53.13 & 20.59 & 38.63 & 2.45 & 1.18 & 1.00 \\
\hline \multicolumn{8}{|l|}{ Chao1 } \\
\hline B_P7 & A_U0 & 43.57 & 7.82 & 29.50 & 5.05 & 2.88 & 0.21 \\
\hline C_M7 & A_U0 & 38.69 & 1.56 & 29.50 & 5.05 & 2.65 & 0.74 \\
\hline D_P14 & A_U0 & 59.35 & 21.67 & 29.50 & 5.05 & 2.55 & 0.69 \\
\hline E_M14 & A_U0 & 39.21 & 2.64 & 29.50 & 5.05 & 3.07 & 0.23 \\
\hline B_P7 & C_M7 & 43.57 & 7.82 & 38.69 & 1.56 & 0.90 & 1.00 \\
\hline D_P14 & C_M7 & 59.35 & 21.67 & 38.69 & 1.56 & 1.34 & 1.00 \\
\hline E_M14 & C_M7 & 39.21 & 2.64 & 38.69 & 1.56 & 0.26 & 1.00 \\
\hline B_P7 & D_P14 & 43.57 & 7.82 & 59.35 & 21.67 & -1.14 & 1.00 \\
\hline B_P7 & E_M14 & 43.57 & 7.82 & 39.21 & 2.64 & 0.92 & 1.00 \\
\hline D_P14 & E_M14 & 59.35 & 21.67 & 39.21 & 2.64 & 1.56 & 1.00 \\
\hline \multicolumn{8}{|l|}{ Shannon } \\
\hline B_P7 & A_UO & 1.48 & 0.45 & 1.43 & 0.39 & 0.18 & 1.00 \\
\hline C_M7 & A_U0 & 2.41 & 0.48 & 1.43 & 0.39 & 2.75 & 0.42 \\
\hline D_P14 & A_UO & 1.83 & 0.69 & 1.43 & 0.39 & 0.91 & 1.00 \\
\hline E_M14 & A_U0 & 2.50 & 0.87 & 1.43 & 0.39 & 2.18 & 0.89 \\
\hline B_P7 & C_M7 & 1.48 & 0.45 & 2.41 & 0.48 & -2.22 & 1.00 \\
\hline D_P14 & C_M7 & 1.83 & 0.69 & 2.41 & 0.48 & -0.98 & 1.00 \\
\hline E_M14 & C_M7 & 2.50 & 0.87 & 2.41 & 0.48 & 0.14 & 1.00 \\
\hline B_P7 & D_P14 & 1.48 & 0.45 & 1.83 & 0.69 & -0.67 & 1.00 \\
\hline B_P7 & E_M14 & 1.48 & 0.45 & 2.50 & 0.87 & -1.80 & 1.00 \\
\hline D_P14 & E_M14 & 1.83 & 0.69 & 2.50 & 0.87 & -0.93 & 1.00 \\
\hline
\end{tabular}

${ }^{1}$ Note: std indicates the standard deviation, $\mathrm{t}$ stat indicates $\mathrm{t}$-statistics 
Supplementary table 2. Cumulative sum scaling analysis of significantly different operational taxonomic units (OTUS) that were identified by 165 rRNA amplicon sequencing of RNA between MacroGard (M) and PBS (P) treatment on day 7 and 14 days post treatment. Significance is based on cumulative sum scaling and fitZIG contrasts.

\begin{tabular}{|c|c|c|c|c|}
\hline OTUid & Phylum & Family & logFC & adj.P.Val \\
\hline \multicolumn{5}{|c|}{ Day $7 M>P$} \\
\hline OTU_33 & Proteobacteria & Rhodocyclaceae & -6.16 & 0.00 \\
\hline OTU_54 & Firmicutes & Streptococcaceae & -4.74 & 0.02 \\
\hline OTU_56 & Firmicutes & Streptococcaceae & -3.20 & 0.04 \\
\hline \multicolumn{5}{|c|}{ Day $7 M<P$} \\
\hline OTU_74 & Proteobacteria & Enterobacteriaceae & 4.99 & 0.00 \\
\hline OTU_27 & Proteobacteria & Comamonadaceae & 5.83 & 0.01 \\
\hline OTU_41 & Firmicutes & Erysipelotrichaceae & 4.38 & 0.01 \\
\hline OTU_39 & Proteobacteria & Xanthomonadales_IS & 4.35 & 0.01 \\
\hline OTU_55 & Firmicutes & Streptococcaceae & 4.38 & 0.02 \\
\hline OTU_70 & Proteobacteria & Rhodocyclaceae & 3.41 & 0.02 \\
\hline OTU_51 & Firmicutes & Bacillaceae & 4.77 & 0.02 \\
\hline OTU_26 & Proteobacteria & Comamonadaceae & 2.88 & 0.03 \\
\hline OTU_57 & Verrucomicrobia & Opitutae_vadinHA64 & 3.78 & 0.04 \\
\hline OTU_12 & Firmicutes & Peptostreptococcaceae & 3.21 & 0.03 \\
\hline OTU_46 & Actinobacteria & Micrococcaceae & 4.00 & 0.04 \\
\hline OTU_53 & Firmicutes & Clostridiaceae_1 & 3.18 & 0.04 \\
\hline OTU_49 & Firmicutes & Clostridiaceae_1 & 2.38 & 0.10 \\
\hline OTU_31 & Proteobacteria & Neisseriaceae & 2.11 & 0.10 \\
\hline \multicolumn{5}{|c|}{ Day $14 M>P$} \\
\hline OTU_69 & Proteobacteria & Oxalobacteraceae & -5.02 & 0.00 \\
\hline OTU_54 & Firmicutes & Streptococcaceae & -4.09 & 0.02 \\
\hline OTU_53 & Firmicutes & Clostridiaceae_1 & -4.19 & 0.03 \\
\hline OTU_79 & Firmicutes & Lachnospiraceae & -3.60 & 0.03 \\
\hline OTU_29 & Firmicutes & Clostridiaceae_1 & -2.80 & 0.04 \\
\hline \multicolumn{5}{|c|}{ Day $14 M<P$} \\
\hline OTU_9 & Proteobacteria & Unknown_Family & 9.71 & 0.00 \\
\hline OTU_26 & Proteobacteria & Comamonadaceae & 6.45 & 0.00 \\
\hline OTU_57 & Verrucomicrobia & Opitutae_vadinHA64 & 5.94 & 0.00 \\
\hline OTU_74 & Proteobacteria & Enterobacteriaceae & 5.16 & 0.00 \\
\hline OTU_27 & Proteobacteria & Comamonadaceae & 5.75 & 0.00 \\
\hline OTU_6 & Proteobacteria & Shewanellaceae & 3.73 & 0.00 \\
\hline OTU_3 & Proteobacteria & Aeromonadaceae & 3.82 & 0.00 \\
\hline OTU_153 & Proteobacteria & Comamonadaceae & 3.83 & 0.01 \\
\hline OTU_114 & Proteobacteria & Rhodocyclaceae & 3.93 & 0.02 \\
\hline OTU_36 & Actinobacteria & Actinomycetaceae & 3.41 & 0.03 \\
\hline OTU_23 & Bacteroidetes & Porphyromonadaceae & 5.40 & 0.03 \\
\hline OTU_4 & Bacteroidetes & Bacteroidaceae & 3.02 & 0.07 \\
\hline
\end{tabular}


Supplementary table 3. Random forest analysis of significantly different operational taxonomic units (OTUS) that were identified by 165 rRNA amplicon sequencing of RNA between MacroGard (M) and PBS (P) treatment on day 7 and 14 days post treatment. Significance is based on using random forest analysis followed by the Boruta feature selection.

\begin{tabular}{lllc}
\hline OTUid & Phylum & Family & Layer importance \\
\hline M $>$ P in both day 7 and day 14 & & \\
OTU_21 & Proteobacteria & Geobacteraceae & 9.77566216 \\
OTU_54 & Firmicutes & Streptococcaceae & 3.772245573 \\
OTU_20 & Proteobacteria & Rhodobacteraceae & 10.69023545 \\
OTU_15 & Firmicutes & Streptococcaceae & 9.265338095 \\
OTU_18 & Firmicutes & Staphylococcaceae & 7.802709367 \\
OTU_11 & Proteobacteria & Pseudomonadaceae & 9.719296364 \\
OTU_33 & Proteobacteria & Rhodocyclaceae & 4.012672004 \\
\hline Unhandeled $>$ M,P & & \\
& & & \\
OTU_23 & Bacteroidetes & Porphyromonadaceae & 7.161350787 \\
OTU_4 & Bacteroidetes & Bacteroidaceae & 8.489835637 \\
OTU_16 & Proteobacteria & Vibrionaceae & 7.138475572 \\
OTU_8 & Bacteroidetes & Porphyromonadaceae & 10.76910099 \\
\hline P>M in both day 7 and day 14 & & \\
OTU_74 & Proteobacteria & Enterobacteriaceae & 6.200066198 \\
OTU_26 & Proteobacteria & Comamonadaceae & 6.506197169 \\
OTU_3 & Proteobacteria & Aeromonadaceae & 4.325133066 \\
\hline
\end{tabular}


1

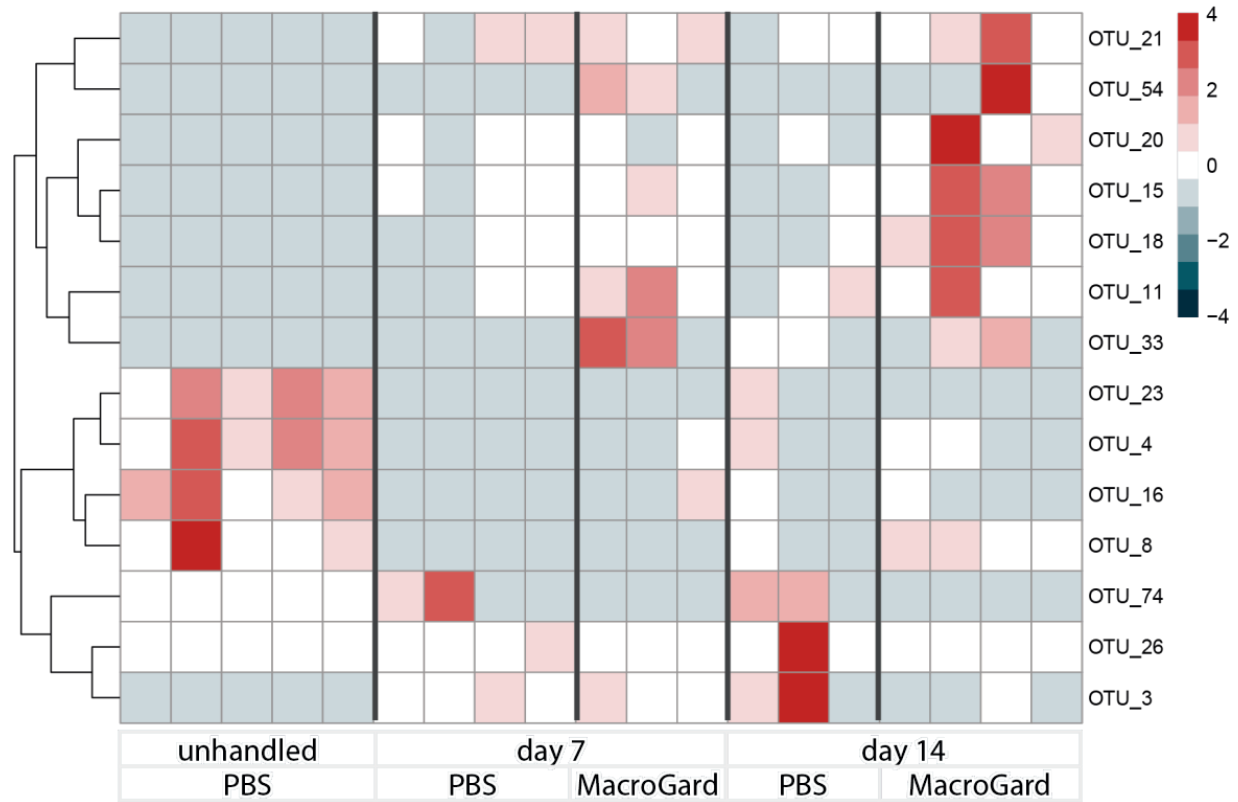

Supplementary figure 1 . Treatment with a single oral $\beta$-glucan gavage alters intestinal microbial community of carp. Significant differences were identified by random forest analysis with Boruta feature selection (average z-scores of 1000 runs). 
A

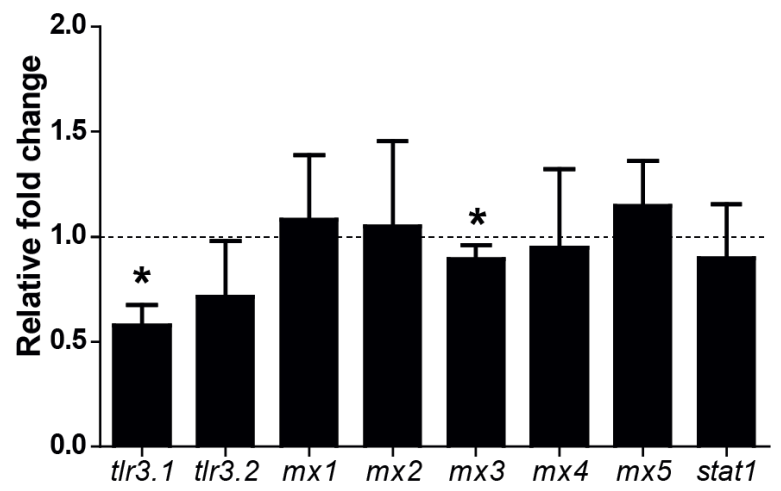

B

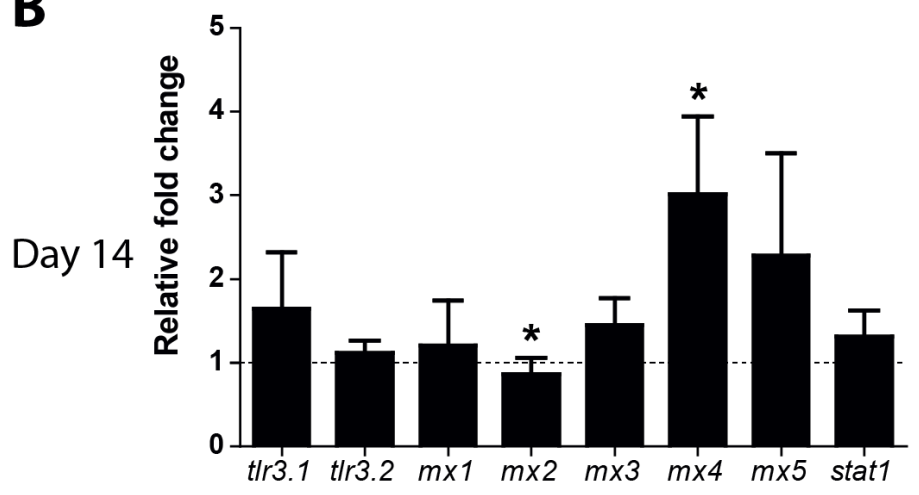

Supplementary figure 2. A. Interferon (IFN) pathway related gene expression seven days post-treatment. Significantly lower expression of t/r3.1 and $m \times 3$ can be observed. A trend towards lower t/r3.2 ( $p=0.084)$ expression can be observed in the treated group. B. IFN related gene expression 14 days post-treatment. Significantly lower expression of $m \times 2$ and increased expression of $m \times 4$ can be observed. A trend towards increased tlr3.2 ( $p=0.096)$ and $m \times 3(p=0.054)$ expression can be observed in the treated group. Asterisk $(*)$ indicates significant difference in gene expression between the group treated with an oral gavage with PBS and the group treated with an oral gavage with

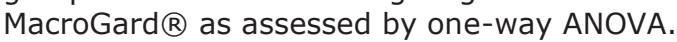





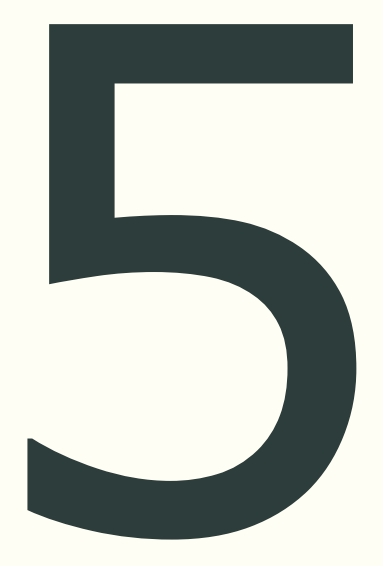

\title{
Genomic and transcriptomic approaches to study immunology in cyprinids: What is next?
}

\author{
Jules Petit, Lior David, Ron Dirks, Geert F. Wiegertjes
}

Developmental and Comparative Immunology 75 (2017), p. 48-62

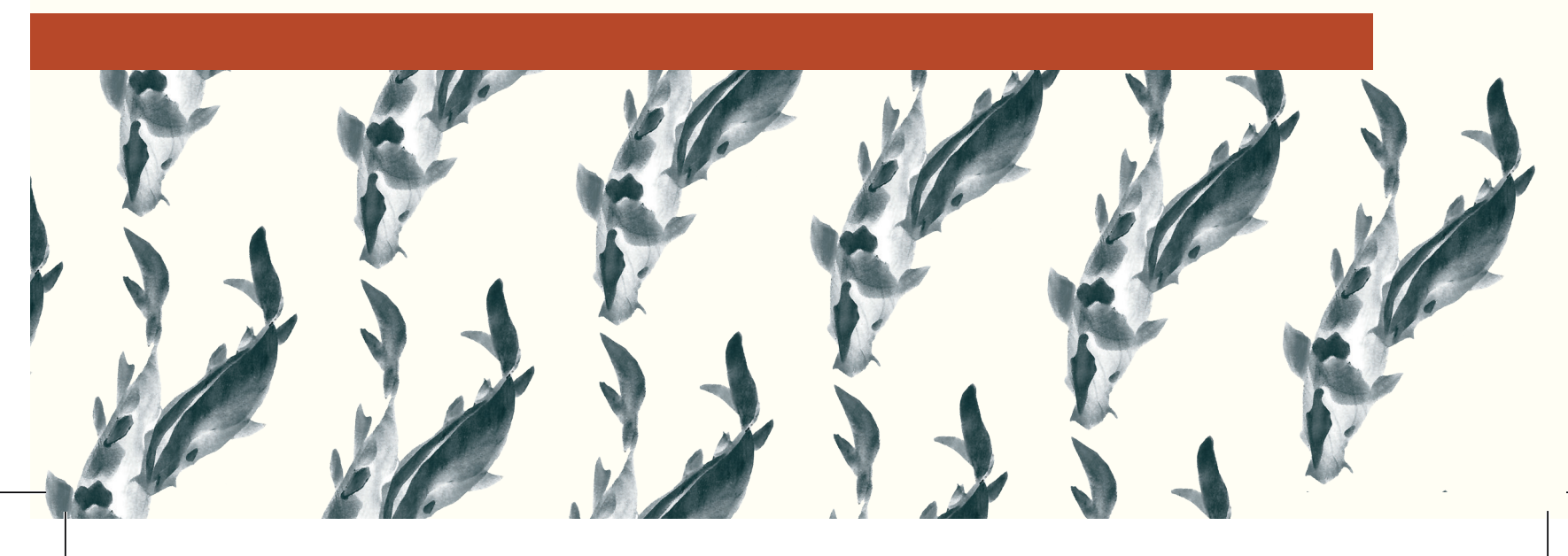




\section{Abstract}

Accelerated by the introduction of Next-Generation Sequencing (NGS), a number of genomes of cyprinid fish species have been drafted, leading to a highly valuable collective resource of comparative genome information on cyprinids (Cyprinidae). In addition, NGS-based transcriptome analyses of different developmental stages, organs, or cell types, increasingly contribute to the understanding of complex physiological processes, including immune responses. Cyprinids are a highly interesting family because they comprise one of the most-diversified families of teleosts and because of their variation in ploidy level, with diploid, triploid, tetraploid, hexaploid and sometimes even octoploid species. The wealth of data obtained from NGS technologies provides both challenges and opportunities for immunological research, which will be discussed here. Correct interpretation of ploidy effects on immune responses requires knowledge of the degree of functional divergence between duplicated genes, which can differ even between closely-related cyprinid fish species. We summarize NGS-based progress in analysing immune responses and discuss the importance of respecting the presence of (multiple) duplicated gene sequences when performing transcriptome analyses for detailed understanding of complex physiological processes. Progressively, advances in NGS technology are providing workable methods to further elucidate the implications of gene duplication events and functional divergence of duplicates genes and proteins involved in immune responses in cyprinids. We conclude with discussing how future applications of NGS technologies and analysis methods could enhance immunological research and understanding. 


\section{Cyprinids are a biologically and economically important family}

The cyprinid family is, economically, one of the most important and biologically, one of the most diverse fish families worldwide. Cyprinid species live in a wide range of different habitats including fresh, brackish and salt water, variable temperatures, water depths and oxygen concentrations. The entire cyprinid family includes approximately 3,000 species, whereas the combined aquaculture production of the 3 most-cultured cyprinid species alone, including grass carp (Ctenopharyngodon idella), silver carp (Hypophthalmichthys molitrix) and common carp (Cyprinus carpio), accounts for almost $20 \%$ of the aquaculture production worldwide (FAO - fisheries and aquaculture ${ }^{1}$ ). Further prominent members of the cyprinid family are species from the Carassius genus including goldfish, the major Indian carps and zebrafish (Danio rerio) (Figure 1 ). Of the most-cultured species; grass carp provides both an important protein source in China and is used in many countries as a biological control for aquatic weeds, whereas silver carp, also a herbivorous species, has been long cultured in polyculture in China. Aquaculture of common carp is found in more than 100 countries and serves several purposes, such as food consumption, but also angling and production of ornamental koi.

Carassius species are of economic importance to aquaculture mostly in Asia, and are a genus commonly known as Crucian carps, although this term is most often specifically used to refer to $C$. carassius. Maybe the best-known member of this genus is the ornamental goldfish (C. auratus), which was bred from the Prussian carp (C. gibelio). The 'major Indian carps' is a common name for three species which together form the backbone of Indian aquaculture: Catla (Catla catla), the most produced in India, Rohu (Labeo rohita) and Mrigal (Cirrhinus mrigala). Although their names indicate each belong to a different genus; their exact phylogenetic relationship is still under debate, particularly true for the position of Catla [see also Figure 1, (238)].

As a prominent and probably the most-studied member of the cyprinid family, the zebrafish has become a widely-accepted animal model species for, among others, human diseases and disorders, drug discovery and screening, and toxicological assessments (239-242). Since the publication of the early teleost genomes, including early builds of the zebrafish genome at the Wellcome Trust Sanger Institute since 2001, but also genomes of the fugu (Takifugu rubripes) (243), green spotted pufferfish (Tetraodon nigroviridis) (244), and medaka (Oryzias latipes) (245), the number of sequenced fish genomes has increased steadily for years, and steeply with the advent of Next Generation Sequencing (NGS, see also

${ }^{1}$ http://www.fao.org/fishery/statistics/en (accessed on: 2017-02-12) 
info Box 1), (246). To date, at least, 108 genome assemblies (among which 30 are mitochondrial and 16 obsolete versions) from 73 different teleost fishes, have been submitted to the National Center for Biotechnology Information (NCBI) ${ }^{2}$. Surprisingly, the number of published genomes of cyprinids is relatively few, and only includes grass carp, common carp, zebrafish, cavefish (Sinocyclocheilus complex), Amur ide (Leuciscus waleckii), and fathead minnow (Pimephales promales). It stands out that, despite their economic relevance, there are no genomes available of silver carp, Carassius genus and the major Indian carps. The relative scarcity of cyprinid genomes is a good example of the difficulties still faced when trying to obtain high quality reference genomes for non-model species, despite the use of NGS technologies. Maybe the gap will be filled as a result of large-scale efforts aiming to sequence and assemble many more teleost genomes such as the $10 \mathrm{~K}$ genome project, which includes several cyprinid species (247), helped by the latest developments in NGS technologies.

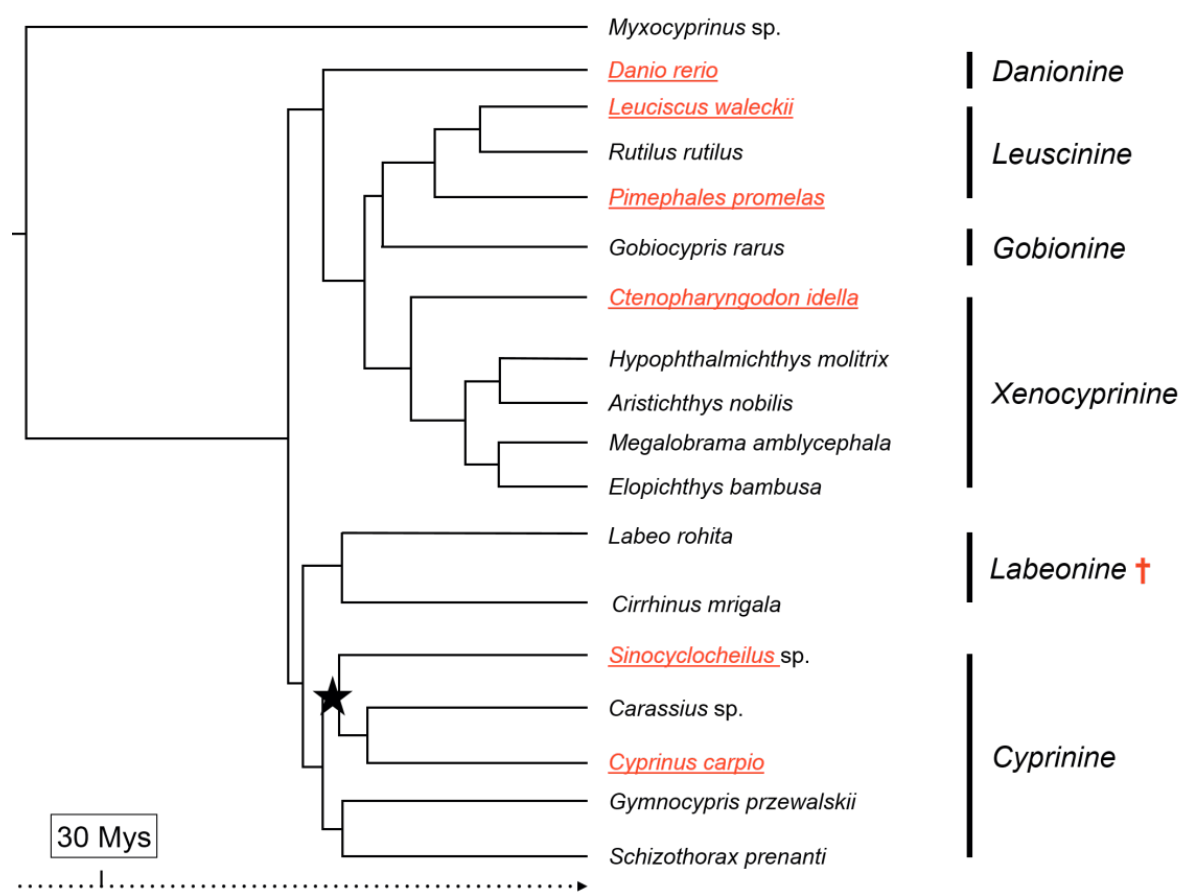

Figure 1. Phylogenetic relationships among key cyprinids with estimates of divergence time based on (238). Myxocyprinus asiaticus was chosen as outgroup. Mys: million years. Species with a have a submitted or published genome assembly are depicted in red and underlined. + : the phylogenetic position of the third major species of Indian carp (Catla catla) is still under debate. The black star represents a likely position for the fourth round of WGD relevant to common carp (Cyprinus carpio) and the Carassius spp., whereas the exact position of the WGD relevant to Sinocyclocheilus spp. is unknown.

2 https://www.ncbi.nlm.nih.gov/genome/browse/ (accessed on: 2017-01-16) 


\section{BOX 1: The generations of sequencing technologies}

First generation sequencing started in 1977 with the introduction of Sanger's "chain termination" technique (248). Sanger sequencing generates individual reads of up to one kilobase in length. The best-known example of a genome sequence assembled from Sanger reads is the human genome $(249,250)$. Second generation sequencing, also originally referred to as next generation sequencing, started around 30 years later, when mass parallelization and miniaturization became possible via pyrosequencing technology (251). Pyrosequencing was incorporated into the Roche 454 sequencer platform and was quickly followed by Solexa/Illumina and SOLiD (Applied Biosystems) sequencing, three competing platforms that use different technologies for parallelization and miniaturization. All three platforms can generate millions of reads simultaneously, ranging in size from less than a hundred (SOLiD) to few hundreds of base pairs (Illumina, Roche 454). Due to the massive throughput, second generation sequencing resulted in a greatly reduced cost price per sequenced base. Draft genome sequences assembled from Illumina reads are often fragmented and the scaffolds contain many sequence gaps, mostly caused by repeat regions that could not be resolved by the short reads. Third generation sequencing refers to very recent techniques based on single molecule sequencing (SMS), which combine generation of long reads with large amounts of sequence information. Examples of platforms are PacBio sequencing and the sequencing device from Oxford Nanopore Technologies. In this review, second and third generation sequencing will be clustered under the term Next Generation Sequencing (NGS). No distinction will be made between second and third generation sequencing, unless explicitly mentioned.

\section{Genome assemblies provide a basis for studying immune responses across cyprinid species}

Sequencing of the zebrafish genome has greatly contributed to the present broad basis of genomic and functional studies not only in the zebrafish itself but in many other fish species, and turned zebrafish into a common reference for many, if not all, immunological studies in teleost fish. In its current assembly version, about 25,000 genes have been mapped on the genome (Table 1 ). In this review, we will mention the zebrafish as reference but will not further discuss genome or transcriptome studies in zebrafish. For a comprehensive overview of the advances in genomic and transcriptomic research in zebrafish we refer to, among others, a recent special issue of Methods in Cell Biology (Volume 135, 2016). It is, however, important to remember here that, being the most-complete and best-annotated cyprinid genome publicly available, the zebrafish provides a reference for new genetic data on any cyprinid fish species.

Common carp most likely is the one cyprinid species of economic relevance that has the most-advanced NGS-based version of the genome. Already in 2011, a preliminary draft combining a de novo assembly based on RNAseq with existing EST and mRNA sequences from GenBank was used to mine for a specific set of immune genes (252). Subsequently, using genomic DNA from a single individual of European genetic background with double-haploid homozygous status, an updated and more complete genome assembly was published (253). The choice for a double-haploid individual greatly facilitated the genome assembly of this 
difficult species of tetraploid origin (254). The assembly published by Henkel and colleagues in 2012 was improved with long read sequencing (Pacific Bioscience) and combined with verification of gene expression, which resulted in a new genome assembly and a total number of 50,527 genes submitted to NCBI (255).

In parallel, a second major genome assembly of common carp was drafted based on a different (Songpu) strain of East-Asian genetic background (256) with 52,610 genes submitted to NCBI. For the latter assembly, contigs were built on single-end sequencing, whereas the scaffold assembly combined BAC-end sequences with paired-end and mate-pair sequences from different sequencing platforms (i.e. Roche 454, Illumina HiSeq and SOLiD). Using 3,470 single nucleotide polymorphisms (SNPs) and 773 microsatellites, a first attempt was made to anchor these scaffolds into 50 linkages groups (256). A comparison of size and number of scaffolds between the different genome assemblies (Table 1) shows differences which might be related to differences in sequencing strategies and platforms, or to the number of individuals sequenced and/or degree of homozygosity of the sequenced individuals. It is also conceivable that genetic differences between common carp of European and East-Asian background (257) might account for some of the differences between common carp genome assemblies. Aligning the two major genome assemblies of common carp revealed preservation of syntenic relationships in $1.13 \mathrm{Gbp}$ (255).

Grass carp genome resources have been relatively few given its economic relevance, and included a genetic linkage map based on 279 markers, identified by a combination of microsatellite and EST-cloning (258). In 2015, a draft genome (Table 1) was published and opened the door to more in-depth genomic investigations. Independent draft assemblies of male and female grass carp resulted in different sizes of the two assemblies. The female genome was annotated, resulting in $>27,000$ genes and was anchored based on the previouslypublished genetic linkage map. The male genome assembly was mainly generated to analyse genetic variation between the female and the male genome sequences. Of the 279 markers, a total of $64 \%$ could be used for anchoring the scaffolds and predicted genes. Despite the genomic progress, the above-discussed assemblies for the common carp and grass carp genomes were primarily based on short-read sequencing and thus, novel long-read technology platforms are expected to further improve the current genome assemblies in the near future.

There are (at least) two genome sequencing projects that have selected particular members of the cyprinid family to study environmental effects, sometimes including effects on immune responses. The Sinocyclocheilus is a genus of cyprinid cavefish which, like other cavefish including the Mexican tetra (not discussed here), have adapted to their unique habitat and thus show differential grades of regression in several features including eyesight, presence of scales and skin pigmentation; all complex phenotypes of evolutionary interest. Three representatives of the Sinocyclocheilus genus, residing in three different habitats, were sequenced and three genome assemblies were generated (Table 1), (259). 
Of particular interest here, based on computational annotation, the study found a correlation between expansion of the immune system and specific habitats of the three species. The cave-restricted species (S. anshuiensis) had fewer copies of particular immune-related genes, including a clear reduction in the copy number of some MHC class II genes (DPA1, DQB1), but more copies of other genes, including particular toll-like receptors (TLR8, TLR18) (259). This study potentially provides a first glimpse of a genetic basis for cave adaptation and the effects of speciation on immune-related gene evolution.

Another example provides the Amur ide (Leuciscus waleckii), of interest because of its evolutionary adaptation to an extreme alkaline habitat (260). The recent annotation of this genome assembly resulted in a total of 23,560 genes. Besides revealing gene family expansion and gene family contraction upon comparison with zebrafish and grass carp, the Amur ide genome assembly also revealed differences between individuals living under alkaline stress and individuals residing in relatively normal conditions (e.g. analysis of genetic diversity revealed copy number expansion in 10 genes between individuals living under alkaline stress and living under normal conditions). As discussed above, genome assemblies for cyprinid species of particular biological interest may allow for a first investigation of processes such as regression, or adaptation to extreme environments. Most certainly, the unravelling of such complex processes will be further facilitated by comparative analyses of genomes of several cyprinid species from diverse habitats.

Fathead minnow (Pimephales promelas) is a cyprinid probably best known for the almost universal use of the epithelioma papulosum cyprini (EPC) cell line for in vitro replication of several viruses (261). This cell line was originally reported to be from common carp epidermal herpes virus-induced hyperplastic lesions, but later recognized as derived from fathead minnow (262), a temperate species of the cyprinid family (Figure 1 ). Although the genome of this cell line may have diverged from its original host species through accumulation of mutations after many in vitro passages, the availability of a whole animal-based fathead minnow genome assembly (263), (Table 1 ) will improve the mapping and facilitate future transcriptome analyses of (innate) immune responses in this popular cell line. The application of NGS to controlled in vitro environments makes transcriptome analysis of cell lines a powerful tool to investigate complex processes such as innate immune responses to virus infection. 

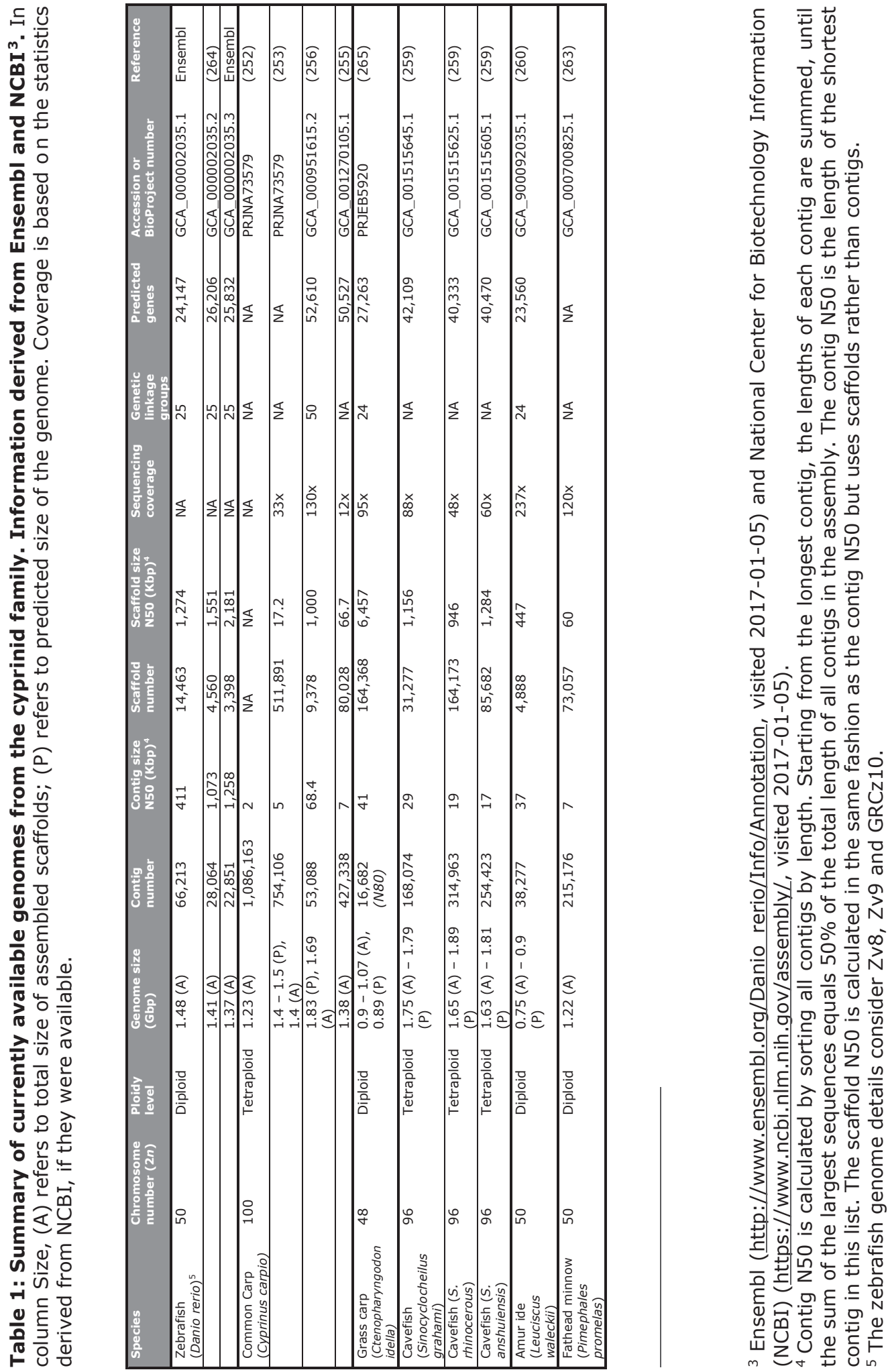
Most of all, it is the combination of several cyprinid genome assemblies that provides a highly-valuable resource for comparative genome analysis. The added value of NGS resources for such comparative analyses is tremendous and will help to study similarities and differences in genome evolution, immune genes and many other processes or mechanisms (266). This is supported by studies observing a high conservation of syntenic relationships after comparative analyses of genome sequences or genetic maps. For example, comparison of grass carp, and Rohu, with zebrafish revealed $88 \%$ of the grass carp genes are located in syntenic blocks (265) and revealed $87 \%$ sequence similarity, on average, between Rohu and zebrafish transcripts (267). Comparative analysis also revealed two crosschromosome rearrangements for two grass carp linkage groups, whereas two zebrafish chromosomes aligned to a single linkage group of the grass carp, suggesting a chromosome fusion. Another example is provided by the recent genetic map of the goldfish, which revealed a clear degree of conservation of synteny, not only between goldfish and zebrafish but also between goldfish and common carp (268). Phylogenetically, however, the Carassius genus is positioned closer to the Cyprinus genus than to zebrafish (Figure 1), $(238,269)$.

A compelling case for comparative genomics based on NGS was made by a recent study of the gene 'TLR4 interactor with leucine-rich repeats' (tril), first detected in an RNAseq data set of common carp (270). Surprisingly, despite a high degree of synteny surrounding the tril gene in the genome of several fish species, assembly Zv9 suggested the absence of tril in the relevant area of the zebrafish genome. Subsequently, detection of three overlapping ESTs for zebrafish tril allowed for the retrieval of the full nucleotide sequence of zebrafish tril. Based on this information the Genome Reference Consortium (GRC) undertook efforts to re-sequence the relevant area absent in the $z \mathrm{v} 9$ assembly. This case provides an excellent example of the added value of accessing multiple genomes, and transcriptomes, from related cyprinid fish species, because it is the collective set that provides the most valuable resource of genetic information for the cyprinid fishes. This would not be possible without the advent of NGS.

Realizing the efforts placed in drafting a genome assembly for, for instance the common carp, it is important to note few points relevant also to other cyprinids. In spite of the great improvements, the genome state is still largely fragmentary (Table 1 ), hence its annotation is still partial and heavily relies on the annotation of the better assembled and better annotated genome of the zebrafish. These imperfections are even more pronounced in genome assemblies of other cyprinids, with potential implications on the interpretation of NGS results that rely on the genome assembly as a reference. Therefore, despite the already invested efforts and the surge in cyprinid genome assemblies, there is still room for further improvement of the genome sequence in order to enhance interpretation of NGS based results. While cyprinid genome assemblies are being improved, considerable insights have already been gained in complex biological systems including immunology, mostly by utilizing the existing genomes and NGS platforms for transcriptome analyses. 


\section{Transcriptome analyses of immune responses - from basal to challenge conditions}

\subsection{Gene expression under basal conditions}

Studying transcription levels and regulation of genes is pivotal for evaluating immune responses and traditionally this was carried out using RT-qPCR on small numbers of genes. As large-scale transcriptome analyses became more feasible and affordable, initially as DNA microarrays, later as NGS-based RNAseq, expression analysis of many genes in parallel has become widely-accepted and commonly-applied. In common carp, a transcriptomic approach was quickly adopted and a de novo assembly based on RNAseq was combined with existing EST and mRNA sequences from GenBank. The combined dataset was mined for a specific set of TIR domain containing immune genes and, using these genes as a reference, 162 contigs from a very fragmented genome could be stitched together into 39 scaffolds (252). De novo transcriptome assemblies have also been employed for data mining and gene identification in crucian carp, identifying 120,000 unigenes of which 6,000 could be assigned to known 'Kyoto Encyclopedia of Genes and Genomes' (KEGG) pathways, including almost 600 genes categorized as 'immune genes' (271). Unigenes can be output of de novo assembly using Trinity or alike; combining reads into contigs, extended with sequence clustering (272). Unigenes should not be confused with genes because upon annotation several unigenes can still result in only a single unique gene. Hence, conclusions based on expression analysis of unigenes should be interpreted carefully as they might not always accurately represent actual gene expression.

In 2014 another de novo transcriptome of the crucian carp was published with the main purpose to identify immune genes (273). Of the total of $>78,000$ transcripts, 7,500 transcripts could be aligned with fish-specific genes from the NCBI database, of which only 77 were deemed immune-relevant. Similar to these studies, a study in common carp employed transcriptome analysis and generated a de novo transcriptome, comprising 36,811 contigs of which 28,000 had a significant hit with the NCBI nr database corresponding to $>19,000$ unique genes, of which 441 were classified as immune system-related by KEGG analysis (274). The relatively low number of immune-related genes identified in the two above-mentioned studies may reflect some of the difficulties faced by de novo assembly of transcriptomes and suggest that the present Gene Ontology (GO) and KEGG analyses are not yet optimized for the investigation of immune responses in fish.

Other NGS-based transcriptome studies also addressed basal (immune) gene expression in cyprinid species such as silver carp (275), blunt snout bream (Megalobrama amblycephala) (276), Schizothorax prenanti (277) and the Tibetan naked carp (Gymnocypris przewalskii), which is of interest due to its ability to cope with hypoxic environments (278-280), but none have so far identified clear immune response profiles. Maybe more powerful examples of NGS-based transcriptome analysis should be sought in studying gene expression under basal 
conditions, such as the transcriptome atlas of common carp (255). Immune response profiles of leukocytes, under basal conditions, can be addressed by NGSbased transcriptomic approaches studying, for example, primary cell cultures of common carp macrophages for validation of purity and confirmation of cell typespecific gene expression as well as polarization states (Wentzel and Wiegertjes, unpublished data), or in unbiased transcriptome analyses of common carp leukocyte cell populations sorted with cross-reactive monoclonal antibodies raised against related, Ginbuna crucian carp (C. auratus langsdorfii) (281), confirming the purity of sorted sub-populations (Embregts and Forlenza, unpublished data).

Given its increased acceptance and application, some constraints of transcriptomic analysis should also be noted. Gene identification, and hence gene expression studies, are still limited by imperfect genome assemblies and often rely on comparison to databases of known genes, significantly limiting the discovery of unique and species-specific genes and transcripts. Furthermore, the technical aspects of NGS sample preparation and analysis could still benefit from further improvement to increase standardization and reproducibility of results. Standardization and quality controls are extremely important prerequisites to meaningful comparative analyses, especially when based on different data sets. Furthermore, the statistical challenge of comparing expression levels of many genes in parallel, creates trade-offs between discovery of subtle changes and false positives. Therefore, at this stage, as NGS-based transcriptome analyses of nonmodel species gains momentum but costs are still considerable, it is important to stress the need for biological replicates, proper controls and rigorous data analyses to obtain reliable and reproducible knowledge. Limiting transcriptome analyses to enrichment of differentially-expressed genes in gene ontology categories and biochemical pathways or gene classifications with KEGG often reduces the outcome to generalized conclusions. Nevertheless, already the currently-available cyprinid transcriptome datasets provide powerful screening tools to guide further detailed studies to understand the functions and roles of genes and immune pathways and are added value for cyprinid immunology.

\subsection{Responses to bacterial challenges}

Besides transcriptome analysis of basal conditions, many studies use a form of exogenous challenge to identify genes of interest with respect to pathogenic conditions. Aeromonas hydrophila is a Gram-negative bacterium of high economic relevance in Asia and has been examined in several cyprinids, which makes it interesting to try and identify putative common transcriptomic responses. In common carp, the kinetics of the antibacterial immune response in spleen at 4, 12 and 24 hours after $A$. hydrophila challenge showed a total of 2,900 differentiallyexpressed genes at least one time point (282). The differentially-expressed genes were further analysed with GO analysis, however no distinct interpretation regarding the kinetics of the challenge and the kinetics of the differentiallyexpressed genes was performed. At a first glance, it appears that a similar number of differentially-expressed genes is upregulated at 12 and 24 hours post challenge, 
however the number of genes significantly down-regulated at 24 hours is higher than at any other time point, potentially indicating the induction of control mechanisms.

In grass carp, $A$. hydrophila challenge revealed 700 differentially-expressed genes in spleen and kidney between early and late moribund fish including an enrichment of several immune-related pathways identified by KEGG and GO analysis. This study also included a small RNA transcriptome to investigate differences in micro RNA (miRNA) between these fish (283). A computational prediction of miRNA targets revealed several inversely-expressed target genes, of which more than half were related to 'immune and disease' pathways. A follow-up study identified 61 conserved and 124 candidate novel miRNAs and uncovered 21 differentiallyexpressed miRNA between the susceptible and resistant grass carp (284). As above, classification as resistant or susceptible was based on time-of-death, without direct evidence for a genetic component involved in the disease resistance, which means the differences in miRNA expression are not necessarily directly related to $A$. hydrophila resistance, although the data do provide information on the onset of different immune pathways elicited by this bacterial infection.

Independent of putative genetic differences, others identified at least 105 differentially-expressed genes involved in the immune response of grass carp to A. hydrophila (285). Also, transcriptome analysis of intestine samples identified 549 differentially expressed genes in the intestine of grass carp 24 hours after challenge with $A$. hydrophila (286). Challenging grass carp with $A$. hydrophila at a temperature 6 degree higher than normal resulted in more than 3,000 differentially-expressed genes, of which 90 genes were immune-related (287). Comparable numbers of immune-related differentially-expressed unigenes ( 88 unigenes) were identified in a transcriptome study addressing the kinetics of the immune response to $A$. hydrophila challenge, up to 72 hours after challenge, again in grass carp (288). The analysis of kinetics of gene expression profiles of this challenge showed a gradual increase over time of the total number of differentially expressed genes, however further analysis of these differentially-expressed genes would be required to make clear conclusions on the kinetics (288). In conclusion, although there are several transcriptome studies on infection of grass carp with $A$. hydrophila, it remains hard to identify common immune responses. A quick comparison of immune responses in the spleen of grass carp and common carp may point at a common regulation of the complement system after challenge with A. hydrophila, an outcome which would require confirmation by further and more detailed analysis. In general, extensive analyses of kinetics of gene expression after pathogenic challenges appear to be missing, in particular regarding time points later than a few days post-infection. Analysis of later time points, in particular, would be expected to provide the presently-missing transcriptomic information on adaptive immune responses.

In Rohu (Indian carp), fish were examined for differential gene expression after selective breeding for resistance to $A$. hydrophila infection (289). Indeed, several 
contigs with differential expression between resistant and susceptible fish could be identified, although the results were confounded by the pooling of RNA samples and the environmental impact (289). Nevertheless, several contigs carrying genes of the major histocompatibility complex, heat shock proteins, serum lectin and glycoprotein genes appeared associated with resistance (290), providing a start for future immunological analyses of immune responses to $A$. hydrophila in Rohu and a start for the selection of strains with improved immunity. Challenging blunt snout bream with $A$. hydrophila resulted in the identification of 150,000 unigenes, of which only a small part was differentially regulated by the bacterial infection (291). Analysis of small RNA libraries generated from liver tissue after $A$. hydrophila infection predicted 61 and 44 differentially-expressed miRNAs at 4 and 24 hours after infection, respectively (292). GO and KEGG analysis of predicted target genes of these differentially-expressed miRNAs suggested an enrichment in the target genes of several immune related pathways, such as TGF- $\beta$ signalling and TLR signalling.

Future transcriptome analyses should integrate multiple studies to increase reliability and generalizability, and identify robust gene expression signatures that otherwise would remain unidentifiable in the individual studies (293). Possibilities for such analyses are growing rapidly with the increasing number of available datasets in public databases, including Gene Expression Omnibus (GEO) (294) and ArrayExpress (295). Meta-analyses, not only within the same species, but also comparative transcriptome analyses among cyprinids might reveal common immune responses. Of course, confounding factors such as age, for example, will need to be taken into account, as illustrated by a transcriptome analysis of 1,700 differentially-expressed genes in the spleen of one-year-old compared with threeyear-old grass carp (296). Luckily, efforts on transcriptome data across teleost species are starting, using identical experimental procedures, to reduce confounding factors and further facilitate comparative analyses (e.g. the Phylofish project (297)). Further, at present the majority of the transcriptome studies in cyprinids that address bacterial challenge have focused on $A$. hydrophila: despite the significant economic implications of these infections, additional transcriptome analysis of bacterial challenges would certainly widen insights into common antibacterial mechanisms.

\subsection{Responses to viral challenges}

Although viral pathogens can have devastating consequences for aquaculture of cyprinids, relatively few studies addressed transcriptome changes following viral challenges. In common carp, the effects of cyprinid herpes virus 3 (CyHV-3, or koi herpes virus) was investigated in spleen at 24 hours after infection (298). Of 70,000 unigenes, a total of 22,000 were differentially regulated in virus-infected tissue, whereas subsequent KEGG analysis resulted in 12,000 differentiallyexpressed unigenes classified into 256 pathways, a quarter of which were mapped into pathways belonging to the immune system. In grass carp, several transcriptome studies addressed immune responses following infection with grass 
carp reovirus (GCRV). Expressed sequence tag sequencing of head kidney of GCRV-infected grass carp and uninfected grass carp resulted in $>22,000$ differentially-expressed tags between the two groups, which mapped to 3,000 unigenes that were further analysed with GO annotation (299). A follow-up study compared multiple tissues and time points of moribund and surviving fish, and noted a global distribution of differentially-expressed genes appeared among all tissues, which might imply GCRV causes a multi-organ disease (300). In addition, transcriptome analysis after GCRV challenge could also identify alternative splicing; $21 \%$ of the total number of genes that were differentially expressed between moribund and surviving fish aligned with 2 or more unique transcripts, suggestive of a considerable amount of alternative splicing in head-kidney and spleen (301).

Of interest, injection with poly(I:C) can also be used to identify anti-viral transcriptomes. Intramuscular injection of Schizothorax prenanti with this synthetic mimic of viral infection induced several differentially-expressed genes in the spleen at 12 hours after injection (302). Last but not least, viral challenge of cell lines, in vitro, eliminates many of the environmental impacts and may thus allow for controlled anti-viral responses. To investigate the kinetics of infection with spring viraemia of carp virus (SVCV), transcriptomes of EPC cells were investigated 3, 6 and 24 hours after infection (303). Differential expression analysis followed by KEGG analysis showed an increase in infectious diseaseassociated genes with an early stress response, but the majority of differentiallyexpressed genes were found at a relatively late ( 24 hours) time point postinfection. Clearly, only few studies addressed transcriptome responses induced by viruses that are potentially threatening cyprinid aquaculture. As with anti-bacterial (immune) responses, it could be of great interest to compare in meta-approach anti-viral immune responses to different viruses in the same species, or against the same virus in different but related species, potentially revealing common antiviral mechanisms.

\subsection{Responses to non-infectious agents}

NGS-based transcriptome analyses have also been used to investigate differential gene expression after non-infectious challenges. Both, intraperitoneal injection with growth hormone (304), or insulin (305), revealed modulating effects on immune responses in the liver which were subtle and would probably have been overlooked with other methods of expression analysis, such as a RT-qPCR, often based on pre-determined sets of genes. Transition from a carnivorous to a herbivorous diet is a challenge to the metabolic flexibility of grass carp $(265,306$ 309), as are the effects of starvation (310), and it could be of great interest to examine effects on the immune system in these datasets. Studies in roach (Rutilus rutilus) analysed the toxicity of several chemicals and downstream effects, revealing disruptions of the regulation of several physiological process, including the immune system (311). Similarly, a study analysing the transcriptome of blunt snout bream gills exposed to ammonia observed, besides differential gene 
regulation, differential regulation of miRNAs (312). Interestingly, an opposite correlation was observed with 250 predicted target genes, showing either significant upregulation if the miRNA was significantly down regulated, or vice versa.

Clearly, the above-discussed complex physiological and immunological relationships have only been touched upon and remain topics of interest that require more detailed investigation by RNAseq, as well as more rigorous and detailed interpretation of RNAseq data in the future. Of course, the new and massive data output characteristic of NGS requires that novel analysis and visualization methods support the full appreciation of future NGS-based studies. As such, it will be more important than ever to focus on true biological insights rather than on the novelty of the approach. Besides these important points, the correct analysis of immune responses in cyprinids is unique because complicated by the effects of a recent WGD event in several family members, affecting degree of conservation and retention of duplicated genes, assembling genome sequences and interpreting transcriptome analyses.

\section{Polyploidy and retention of duplicated genes complicate analysis of immune responses in cyprinids}

The variation in ploidy levels between cyprinid species $(238,313)$ makes this family highly interesting biologically, with at least, diploid, triploid, tetraploid and hexaploid family members. All teleost fishes are generally believed to have undergone three whole genome duplication (WGD) events, two at the root of the vertebrate lineage $(314,315)$ and one teleost-specific WGD (316-318). Several cyprinid species have undergone an additional WGD (thus fourth round, see Figure 1 ), which is estimated to have occurred very recently in evolutionary terms, with estimates suggesting only 12 million years ago (MYA) for common carp (319-321). In comparison, the additional WGD event that occurred in the salmonid ancestor has been dated back to an earlier time of 80 - 95 MYA, approximately (322-324).

Genome duplication can occur by either auto- or allopolyploidization, terms already coined in 1926 (325). Autopolyploidization is the duplication of the species' own genome whereas allopolyploidization is the doubling of chromosomes following an interspecific hybridization. The two types of genome duplications are different not only by the process but also by the outcome with respect to the similarity in sequence of the duplicated genes following the duplication, which is higher in autopolyploid individuals. Furthermore, while in allotetraploid species the inheritance follows a disomic pattern like in diploids, in autotetraploid species the inheritance often follows a full or partial tetrasomic pattern, which further increases gene conversion and non-allelic recombination between duplicated loci, as was exemplified for the MHC class I and II genes in the polyploid frog Xenopus ruwenzoriensis (326). Salmonid species provide a clear example for 
autopolyploidization, whereas common carp and Carassius spp. provide examples for allopolyploidization. Most likely, the ancestor of common carp and the Carassius genus experienced a WGD, after which their speciation occurred. This hypothesis is supported by an overall 2:2 conservation of genome-wide synteny between common carp and goldfish $(268,327,328)$. In either case, auto- or allopolyploidization through a WGD event instantly doubles the gene copy number, creating both sequence and functional redundancy, which alleviates the evolutionary constraints on gene sequences and allows their faster evolution (314) (duplicated genes, see also box 2). Based on studies in yeasts, plants and last but not least the polyploid Xenopus family of clawed frogs, it is hypothesized that a WGD is generally followed by re-diploidization; the polyploid individual will retain the duplicated set of chromosomes but 'rapidly' lose one copy or the other (329332).

\section{BOX 2: Duplicated genes evolutionary terminology}

Genes can have multiple copies that share sequence similarity and therefore, possibly also common functionalities. The terms used to describe gene copies come from their evolutionary history. The terms homologue, orthologue and paralogue are often misused or confused. To circumvent further confusion this review will use the definitions as proposed by (333). The term homologous genes refers to genes that show sequence similarity because they share a common evolutionary ancestor. Paralogues and orthologues are subdivisions of homologues based on how these copies have evolved. Orthologous genes are genes that originate from a single ancestral gene in the most recent common ancestor, but have diverged due to speciation and diversification events. Paralogous genes are genes that originate from gene duplication, usually within one species (ancestral or extant). Co-orthologues refers to two or more genes that are collectively orthologues to one or more genes in another species, thus co-orthologues originate from a single ancestral gene in the most recent common ancestor. For example, zebrafish NOS2a and NOS2b are paralogues of one another, and NOS2ba and $N O S 2 b b$ in common carp are paralogues in carp and co-orthologues of NOS $2 b$ in zebrafish. Ohnologues refer to paralogous genes that have arisen due to whole genome duplication, named such in honour of the scientist (Ohno) who conceived the theory on the evolutionary roles of duplication and fate of duplicated genes (314).

Commonly, there are three evolutionary fates described for duplicated genes that shape their status in the genome: non-functionalization (334), subfunctionalization (335) and neo-functionalization (314), (see also info Box 3). Functional divergence of duplicated genes (sub- and neo-functionalization), in particular, can be a strong driver underlying the expansion of particular gene families, and is thus very relevant for studies unravelling immune responses in cyprinid fishes. An extensive investigation of re-diploidization was recently published along with the generation of the Atlantic salmon (Salmo salar) genome assembly (324). Although a study in salmonids, it can help understand the process of re-diploidization and aid future studies in cyprinids. The authors show that $20 \%$ of the genes duplicated as a result of the ancient teleost-specific WGD, and 55\% of the genes duplicated as a result of the salmonid-specific WGD, are still retained as two functional copies, without bias for any of the two WGD events. This estimate 
is in line with a previous estimate in rainbow trout (Oncorhynchus mykiss) (323). Further investigation of the fate of duplicated genes in salmon revealed a high degree of neo- instead of sub-functionalization with over $60 \%$ of the investigated duplicated gene pairs showing signatures of tissue-dependent divergent regulation, signifying the expressional divergence of ohnologues.

\section{BOX 3: Fate of duplicated genes}

Following gene duplication, most notably due to whole genome duplication, evolutionary constraints on sequence evolution are reduced and therefore, in general, duplicates evolve faster than singletons. Furthermore, polyploidy is a transient state and duplicated genomes go through a re-diploidization evolutionary process involving loss of duplicated gene copies. The loss of different copies of duplicated genes in different species is referred to as divergent resolution (318). Currently, three different divergence paths for duplicated genes are widely accepted (336). Non-functionalization refers to the situation where one copy becomes a pseudogene due to mutations, eventually leading to gene loss. Neo-functionalization refers to the situation where one copy acquires a mutation that confers a new function, which was not part of its ancestral gene function, and the other copy retains its original function. Sub-functionalization refers to the situation where subsets of the ancestral functions are divided between the copies. While non-functionalization explains the loss of gene copies, neo- and sub-functionalization are explanations for why many gene copies are retained. This evolutionary rational stresses the importance of studying the functions of (immune) gene families and gene copies in a copy specific manner.

In common carp, retention of duplicated genes in the most conserved genomic regions appears high (>92\%), although examined in only 736 pairs and in doubleconserved syntenic (DCS) regions (337). DCS regions are genomic blocks containing genes positioned on one chromosome in a diploid reference species (here, diploid zebrafish), but on two different chromosomes in the duplicated (here, tetraploid common carp) genome. Retention rate can be over-estimated by focusing the analysis on such stretches of highly similar regions, the DCS regions. Indeed, a previous analysis based on microsatellite loci estimated the retention rate in common carp to be lower with 60 - 70\% (319). Regardless, sequence similarity of the analysed duplicated genes was invariably high ( $>90 \%)$, both at nucleotide and amino acid level (337). Also, the regulation of these highly conserved duplicated genes was relatively conserved, although less conserved than their coding sequence similarity, showing co-expression of duplicated genes in most cases. However, about half of the co-expressed genes investigated showed significant expression divergence in one or more tissues. Besides divergent expression, a proportion of the analysed duplicated genes showed functional divergence, implied by functional domain annotation compared to zebrafish, highlighting once more the importance of these duplicated genes and asking for a critical analysis of immune responses in polyploid species.

The consequences of WGD events and associated effects of polyploidization can have their impact on proper analysis of immune responses. Divergent resolution is a process that operates independently in different species and will result in 
different retention of duplicated (immune) genes even between related species, as signified by species-specific genes found in grass carp (265), Rohu (290) and common carp (253). These species-specific genes are easily filtered out by automated annotations, but might be highly interesting from a comparative point of view. Also, tissue-specific expression of duplicated (immune) genes requires careful examination to prevent misinterpretation of immune responses or, when based on examination of only one of the two paralogs, even wrong conclusions. Whereas NGS techniques can rapidly improve reference and other currently available genomes, it can be prone to errors as a result of polyploidy effects: high similarity between duplicated regions can obscure proper genome assembly. Indeed, indications for so-called assembly collapses in homologous regions with the highest sequence similarity $(>95 \%)$ were observed in the salmon genome (324). These regions were highlighted by an increased read depth alignment and reduced scaffold length and accounted for almost $10 \%$ of the chromosome positioned sequences, signifying the difficulties in assembling genomes of polyploidy species.

There is some relief to be found in the knowledge that recent advancements in NGS will help to further resolve difficulties in transcriptome analyses and genome assemblies, including mapping of duplicated genes which, currently, is still largely based on short reads prone to error, signifying the need for high-throughput, long read sequencing platforms. Further, genome assemblies can also be improved by employing existing genetic variance within species to specifically study each gene copy, generate genetic maps, and anchor genomic scaffolds.

\section{Employing genetic variation to improve genome assemblies and study specific gene copies}

Ideally, we wish de novo assembly of NGS sequencing data to result in a perfect genome assembly. However, with the current technologies, de novo assembly typically yields many contigs and a fair number of assembly errors, reflected by the fragmented nature of the current cyprinid genome assemblies (Table 1 ). In principle, following de novo assembly, scaffolding (i.e. the process of putting together contigs in the order of the chromosome) could be improved by comparative mapping to other fish genomes, but this becomes more complicated depending on species diversity in both sequence and genome organisation. In addition, correct assembly is even more complicated for polyploid species with duplicated genomes: the higher the sequence similarity between paralogous regions is, the more difficult it becomes to assemble short sequence reads into their correct contig/scaffold. Furthermore, to avoid sequence mismatches and allow for a more stringent assembly of contiguous reads, genome sequencing is often performed on DNA of a highly inbred homozygous individual. However, this particular approach is methodology-driven and does not always fully respect the biology of the species. For duplicated genomes such as found in several cyprinids, 
residual allelic variation can confound genetic variation between paralogs and complicate the genome assembly. One solution to assist scaffolding is to use genetic linkage maps which build on identifying large numbers of polymorphic markers and ordering them one relative to the other based on their segregation pattern in families. While paralogous polymorphisms in parents show independent assortment in progeny, genetically-linked markers are co-inherited and thus, cosegregate in the mapping population, allowing correct assignment of the DNA marker to linkage groups, even in duplicated genomes (Figure 2), (319). This is why using genetic linkage maps to improve scaffolding and genome sequence assembly is even more valuable for polyploid species.

The possibility to obtain large amounts of sequence information by NGS not only allows for sequencing genomes of single reference individuals but also permits comparison of different individuals and identification of genetic polymorphisms within and between populations (246, 338-340). In principal, to detect and genotype polymorphisms, NGS is usually applied to libraries with reduced genomic representation such that multiple individuals can be reliably analysed with sufficient coverage $(341,342)$. Depending on the specific protocols, these methods typically yield anywhere between thousands to several ten-thousands DNA markers per studied population in a cost-effective manner (Figure 2). Based on this unprecedented resolution, both existing and new research questions in multiple fields such as ecology, population genetics, quantitative genetics, breeding and immunology could be addressed with higher accuracy (339, 343346). Thereby, NGS platforms have revolutionized not only genome and transcriptome analyses but also identification of genetic variation and the use of DNA markers. In the future, newly-developed sequencing technologies are expected to succeed in producing reliable contiguous genome sequence assemblies, which will relegate the need for genetic linkage maps. At this moment, however, genetic linkage maps can already greatly improve scaffolding and genome assembly, especially of non-model species. Since in genetic linkage maps, marker position and marker order are determined based on recombination data in segregating families, the same markers can also be mapped to existing contigs and scaffolds allowing ordering of the latter (324, 347-349). Because the large numbers of DNA markers typically show a genome-wide distribution, this generally results in the constructing of dense genetic linkage maps with a total number of linkage groups corresponding to the number of chromosomes, which is the final goal for the genome assembly.

The utility of DNA markers and genetic linkage maps go beyond improving scaffolding of the genome sequence. Having numerous DNA markers organized into a dense genetic map help in studying the function of duplicated genes, regardless whether a gene had been individually duplicated or duplicated as part of a segmental or whole-genome duplications. Often, the presence of polymorphic gene sequences in the genome assembly suggests a gene has multiple copies. Distinguishing multiple copies based on their sequence variation allows for studying more accurately their genomic organization, positioning, expression 


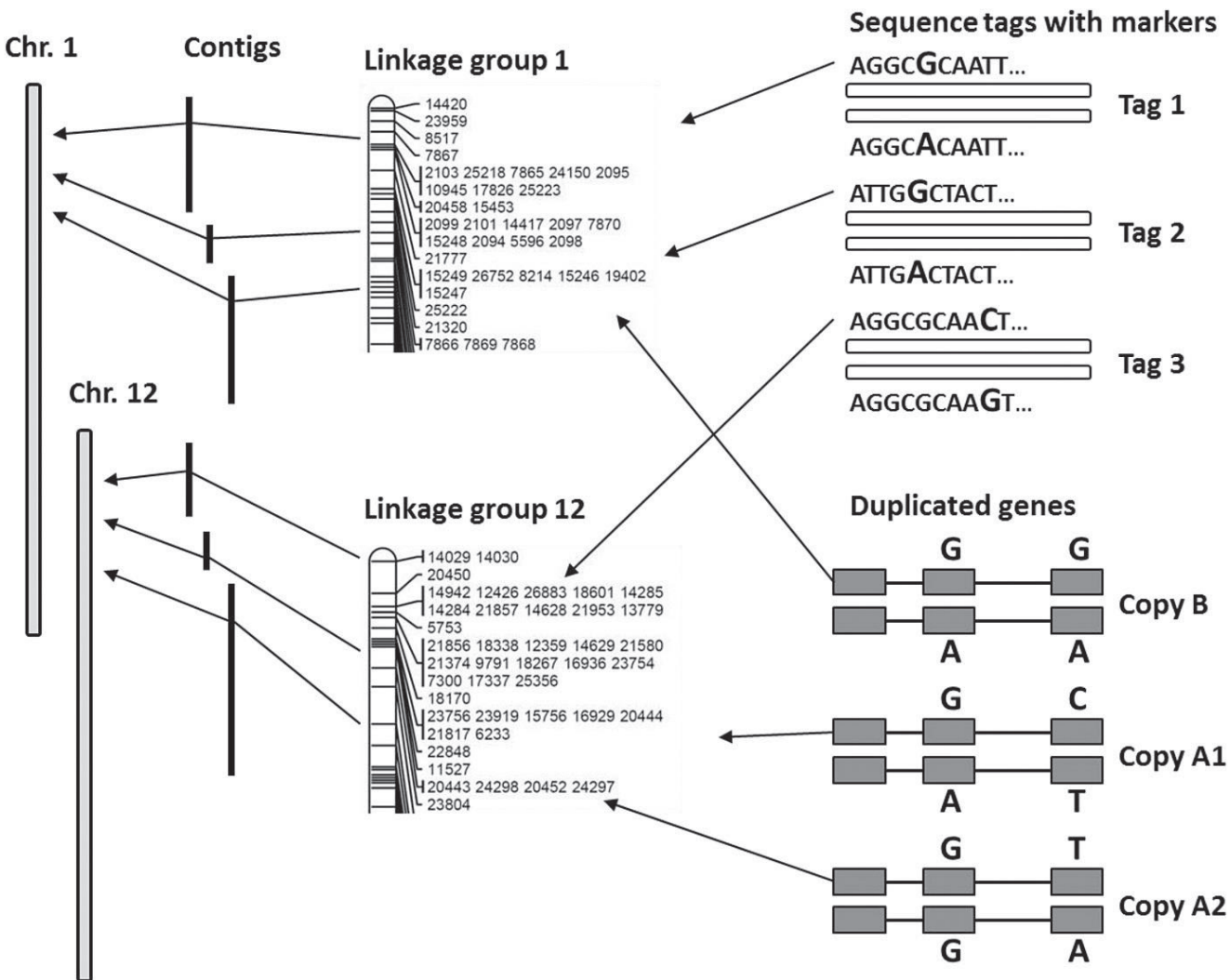

Figure 2. Improving scaffolding and resolving genomic organization of duplicated genes using DNA markers. NGS can generate information on many polymorphic markers by sequencing reduced representation libraries of multiple individuals. Information on the segregation of these markers in the mapping population allows for the construction of dense genetic linkage maps that group markers into linkage groups, and order them within each group. Depending on the number of recombination events, several markers might be assigned to the same position. The marker segregation pattern in the mapping population allows for correctly assigning tags with similar sequence, such as tags 1 and 3, each to a separate linkage group whereas often, in de novo sequence assemblies, such short reads might be collapsed to the same position. Similarly, any set of duplicated genes that has polymorphisms between the alleles of the same copy and between the different copies, can be assigned to linkage groups if copy-specific markers have been developed and genotyped. DNA markers are part of short sequence tags and these are used to identify the contigs that contain each of the markers. Based on the linkage groups, contigs with markers in them are grouped and the order of the markers is used to scaffold the contigs into chromosomes. Note that for both, sequence tags and duplicated gene copies, the polymorphisms in them in combination with genetic linkage mapping allow for determining their genomic organization and correct evolutionary history. In this example, copies A and $B$ are part of a duplication of all or part of the chromosome, while copies A1 and A2 are local tandem duplicates. 
regulation and function. For example, interleukin 10 (IL10) was found to have two copies in the genome of the common carp (350). Taking advantage of the polymorphism between these copies, their functional divergence was shown by association with disease resistance and at the level of gene expression $(350,351)$. Although common carp IL10 was previously studied as a single gene (352), to date we appreciate that each copy might function differently and thus, interrogating duplicated genes in a copy-specific manner is essential for a complete understanding of immune responses. Furthermore, using IL10a- and IL10bspecific polymorphisms, the two copies were shown to segregate independently, and are thus not the result of a tandem duplication but locate to different chromosomes $(253,255,256)$. Often, genes that locate in tandem in the same genomic region are subject to regional regulation (353) and thus, might diverge more slowly in their regulation. Both copies of IL10 share good similarity in their upstream regulatory sequences but do not share genomic location. Resolving the genomic organization of IL10 in the polyploid common carp and studying its copies in a copy-specific manner is just one example of using polymorphism information from genetic linkage maps with genome assembly to improve understanding the function of the immune system.

\section{What is next?}

Further technological developments will continue to have major impacts on the improvement of current and new (cyprinid) genome assemblies. By now, first and second generation sequencing, requiring clonal amplification of the template molecules prior to the actual sequencing, may be considered major developments of the past four decades. The introduction of single molecule sequencing (SMS) marked essentially the start of third generation sequencing and will continue to have a major impact during the next decade. Pioneered by Helicos Biosciences (354), the actual breakthrough came in 2011 when Pacific Biosciences launched their single-molecule real-time (SMRT) sequencing platform onto the market. The PacBio technology uses single DNA polymerase molecules to produce long reads from single molecule templates. Initially, maximum read lengths were in the range of a few kilobases and PacBio data were mainly used to support secondgeneration-sequencing-based de novo assembly of bacterial genomes (e.g. (355)). The average error rate of PacBio reads is relatively high (11-15\%), but individual reads can be corrected using, for example, more accurate Illumina reads. Alternatively, a reliable consensus sequence can be deduced from high coverage ( $>50$-fold) PacBio reads via bioinformatics tools. The latest PacBio systems show much improved average read lengths of more than ten kilobases, which allows resolving long genomic repeat regions that cannot be unravelled using short Illumina reads. For example, de novo assembly of the human genome sequence using PacBio reads only has already contributed to a much-improved version of this reference genome (356). A complementary platform for scaffolding contigs is provided by the Irys $\AA$ system of BioNanoGenomics. This system uses microfluidics to run extremely long (up to $1 \mathrm{Mb}$ ) individual DNA fragments in their linear 
form through long and narrow glass channels to produce an optical map based on the relative positions of fluorescently-labelled restriction enzyme sites. The pattern of restriction sites in the optical map can subsequently be compared to in-silico predictions of restriction sites based on the available genome assembly, together facilitating scaffolding of the contigs ideally resulting in more contiguous genome sequences (e.g. (357)).

Currently, maybe the most promising development is linked with the commercial launch in 2014 of a small sequencing device by Oxford Nanopore Technologies, named MinION. This technology is completely different from all previous sequencers and is based on individual DNA molecules that are guided through protein nanopores; the typical distortion of the electric current as different DNA nucleotides pass through the nanopores is immediately translated into sequence reads. The MinION device is not only small, cheap and mobile but also allows for the production of hundreds of thousands of very long sequence reads in a single run. With this technology, limitation of read length appears to be restricted by the integrity of the DNA after purification and the error rates, but single reads of about $400 \mathrm{~kb}$ have already been reported using the current chemistry. This technology and its future development will greatly facilitate de novo assembly of high quality genome sequences for any organism. The first bacterial genome assembled from nanopore sequencing reads alone was published in 2015 (358) and complete vertebrate genomes assembled from nanopore reads alone are now emerging (359).

The PromethION, a larger version of the MinION with a predicted output of more than five terabases per day, will soon enter the market and further impact NGS applications. It is expected that assembly of intact haploid genome sequences for any organism will soon become possible and, especially relevant for cyprinids, identification of orthologous genes based on homology and synteny will become a challenge of the past. Also, these technologies hold a promise for identification of many sequence variants by sequencing genomes of multiple individuals from the same species. Once this will be made possible, continuous reference to genomes of model species such as the zebrafish, required to guide scaffolding and annotation of genomes of new species, will no longer be essential. These independent genome assemblies thus will also identify species- and even individual-specific features such as genomic rearrangements, gene duplications and repetitive elements variation, allowing even more accurate studies on the functions of gene families. Of course, some challenges will still remain even with very long reads, such as stitching correctly the chromosomes of heterozygous individuals in populations containing allelic variation affecting gene function. No matter the exact technology, the present rapid increase in high-quality genome assemblies will further facilitate investigation of evolutionary effects of WGD events on fish genomes, and thus be of great importance to the understanding of the fish' immune system. 
Advances in NGS are rapidly leading to a flood of RNA sequencing data, highlighting the crucial importance for new analysis and visualization methods to be employed by bio-informaticians and biologists to critically interpret NGS-based studies and properly address biological questions. In spite of the relatively unbiased approach of full transcriptome analyses, the current standard of analysis is often biased, because it is restricted to the listing of regulated pathways identified based on sub-optimal allocation of genes to pathways identified in mammalian species or, at best, in zebrafish. Reflections on the biological significance of differentially expressed pathways for immune responses often remain elusive. Furthermore, such pathway analyses are commonly based on diploid species, obscuring information on duplicated genes, potentially leading to misinterpretation. Finally, most of the transcriptome studies in cyprinid species, as discussed here, addressed immune responses over periods of a few days, leaving opportunies for future research on the dynamics of immune responses over longer periods of time. A systemic analysis of gene expression profiles over periods of days to weeks should highlight the transition from innate towards adaptive immune responses and could reveal intriguing patterns of gene expression during the adaptive phase.

Maybe one of the most exciting immunological applications of NGS will come from sequencing single cells, which application already revealed cell-to-cell variation in seemingly homogenous cell populations of human leukocytes, revealing both functional and regulatory diversity within a cell fraction $(360,361)$. Single-cell sequencing of mRNA has recently been applied to zebrafish, revealing a highlycoordinated transcriptional framework through which hematopoietic cells progress upon their commitment and differentiation towards thrombocytes (362). Furthermore, single-cell mRNA sequencing in zebrafish revealed new subsets within specific epicardial cells and thereby facilitated identification of new potential markers (363). Single-cell sequencing would be a highly-suitable approach for further investigation of the theory on micro-heterogeneity in apparently clonal immune cells. Very likely, NGS-based analyses will also shed light on the mechanisms proposed for the cell alacrity (364), including genome-wide epigenetic modifications, which are increasingly recognized as important regulators of immune responses.

We also foresee great potential to apply the latest NGS approaches for detailed analyses of tissue-specific sub-regions such as lymphocyte aggregates in spleen after infection (365), lymphoid aggregates in nasal immune tissue (366), regions in the hindgut important for antigen uptake $(367,368)$, interbranchial immune tissue (369), and so forth. Investigation of gene expression in these specific regions and immune tissues, either under basal conditions or after bacterial or viral challenge, might reveal details of immune responses to a depth not possible before. These analyses will provide indications towards the organisation and regulation of lymphoid aggregates and tissues during homeostasis and pathogenic challenge in species, such as the cyprinids, lacking highly-organized lymphoid tissues. Another highly interesting application of NGS is dual transcriptome 
analysis, where transcriptional changes of both pathogen and host are analysed simultaneously (370) and may improve our current understanding of host pathogen interactions. Last but not least, NGS will facilitate studies into biological effects of (immune) gene duplications. Variation in ploidy, in combination with a relative conservation of genome organisation and synteny, make the cyprinids a family particularly suitable to this purpose. To this end, novel and high quality genomic and transcriptomic data should prevent misinterpretations of transcriptomic data by a proper distinguishing of paralogous regions. Thereby, NGS will prove essential in studies on genome duplication effects on immune responses in cyprinids.

\section{Acknowledgements}

Pierre Boudinot is gratefully acknowledged for his contribution to the phylogenetic tree of the cyprinid family. LD gratefully acknowledges Wageningen Institute of Animal Sciences (WIAS) for a research fellowship supporting this work. JP and GFW gratefully acknowledge that research leading to this review was funded by the Netherlands Organisation for Scientific Research and São Paulo Research Foundation, Brazil (FAPESP) as part of the Joint Research Projects BioBased Economy NWO-FAPESP Programme (Project number 729.004.002). GFW and LD were supported, in part, by the European Commission under the Work Programme 2012 of the Seventh Framework Programme for Research and Technological Development of the European Union (Grant Agreement 311993 TARGETFISH). 



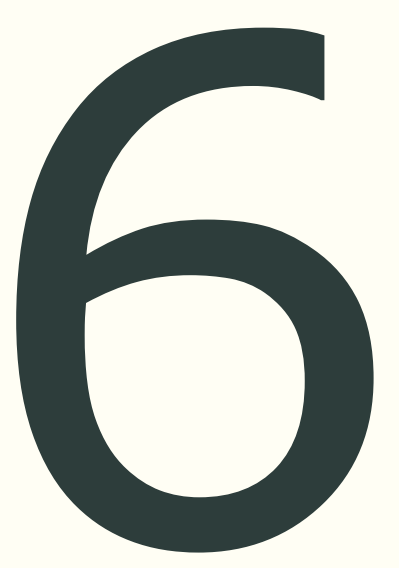

\section{Studies Into $\beta$-Glucan Recognition in Fish Suggests a Key Role for the C-Type Lectin Pathway}

Jules Petit, Eric C. Bailey, Robert T. Wheeler, Carlos A.F. de Oliveira, Maria Forlenza, Geert F. Wiegertjes

Frontiers in Immunology 10 (2019), article 280

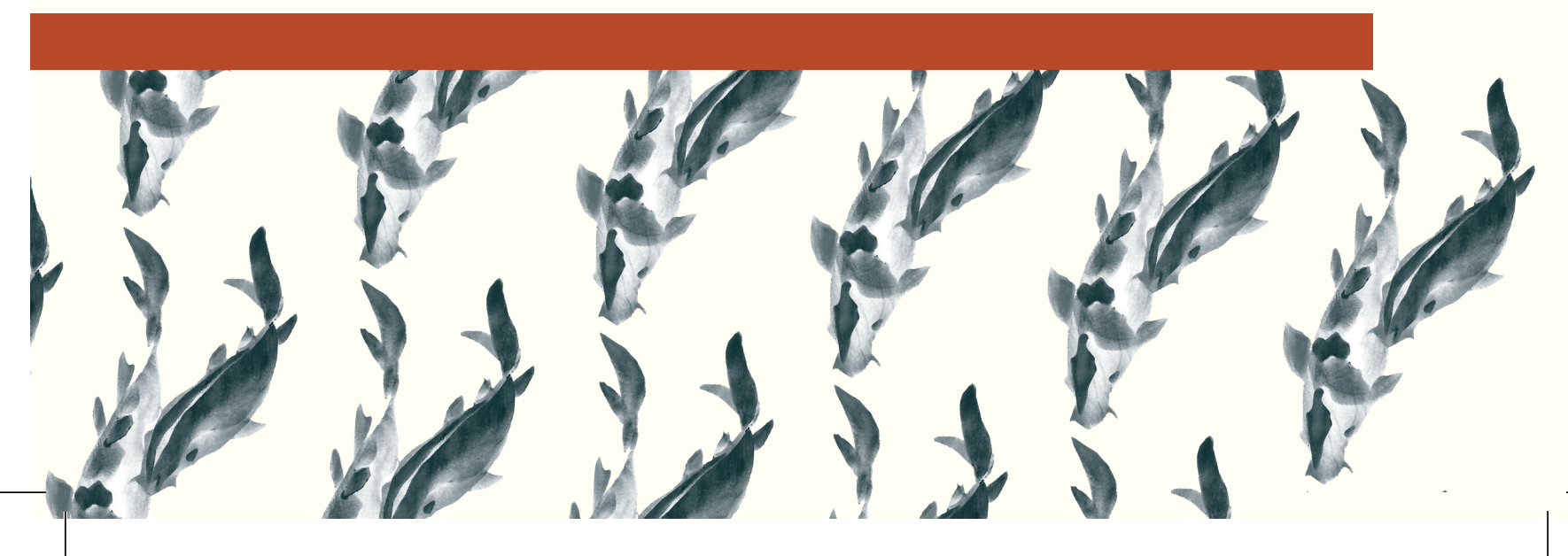




\section{Abstract}

Immune-modulatory effects of $\beta$-glucans are generally considered beneficial to fish health. Despite the frequent application of $\beta$-glucans in aquaculture practice, the exact receptors and downstream signalling remains to be described for fish. In mammals, Dectin-1 is a member of the C-type lectin receptor (CLR) family and the best-described receptor for $\beta$ glucans. In fish genomes, no clear homologue of Dectin-1 could be identified so far. Yet, in previous studies we could activate carp macrophages with curdlan, considered a Dectin-1-specific $\beta$ - $(1,3)$-glucan ligand in mammals. It was therefore proposed that immune-modulatory effects of $\beta$-glucan in carp macrophages could be triggered by a member of the CLR family activating the classical CLR signalling pathway, different from Dectin-1. In the current study, we used primary macrophages of common carp to examine immune modulation by $\beta$-glucans using transcriptome analysis of RNA isolated six hours after stimulation with two different $\beta$-glucan preparations. Pathway analysis of differentially expressed genes (DEGs) showed that both $\beta$-glucans regulate a comparable signalling pathway typical of CLR activation. Carp genome analysis identified $\mathbf{2 3 9}$ genes encoding for proteins with at least one Ctype Lectin Domains (CTLD). Narrowing the search for candidate $\beta$-glucan receptors, based on the presence of a conserved glucan-binding motif, identified 13 genes encoding a $\mathbf{W x H}$ sugar-binding motif in their CTLD. These genes, however, were not expressed in macrophages. Instead, among the $\beta$-glucan-stimulated DEGs, a total of six CTLD-encoding genes were significantly regulated, all of which were down-regulated in carp macrophages. Several candidates had a protein architecture similar to Dectin-1, therefore potential conservation of synteny of the mammalian Dectin-1 region was investigated by mining the zebrafish genome. Partial conservation of synteny with a region on the zebrafish chromosome 16 highlighted two genes as candidate $\beta$-glucan receptor. Altogether, the regulation of a gene expression profile typical of a signalling pathway associated with CLR activation and, the identification of several candidate $\beta$-glucan receptors, suggest that immune-modulatory effects of $\beta$-glucan in carp macrophages could be a result of signalling mediated by a member of the CLR family. 


\section{Introduction}

Immunomodulation by $\beta$-glucans has been widely studied in teleost fish. Regardless of the administration route or fish species, $\beta$-glucan administration often has an immune stimulatory effect and can result in increased resistance to both viral and bacterial infections (reviewed by: (19-21). For both, mammalian vertebrates $(43,185)$ and invertebrates $(89,184)$, specific mechanisms responsible for $\beta$-glucan recognition and/or downstream signalling have been described. Yet for teleost fish, despite the frequent application of $\beta$-glucans in aquaculture practice, the exact mechanisms underlying the induced effects are ill described.

In mammals, several non-exclusive pathways play a role in the recognition and down-stream signalling after $\beta$-glucan stimulation, however the best-described $\beta$ glucan receptor is Dectin-1, also known as C-type Lectin domain Family 7 member A (CLEC7A). Dectin-1 is a C-type lectin super family $V$, NK cell receptors transmembrane receptor with a single carbohydrate recognition domain (CRD) and a cytoplasmic tail containing one ITAM motif (27-29). Dectin-1 is predominantly expressed on cells from both the monocyte/macrophage and neutrophil lineages, where it acts as the major $\beta$-glucan receptor (30). Ligation of $\beta$-(1,3)-glucan to Dectin-1 is dependent on two specific amino acid residues in the CRD; tryptophan (W) and histidine $(\mathrm{H})$, separated by a third residue (WxH motif) (31). Additionally, a tyrosine ( $Y$ ) residue separated from histidine in the CRD by 4 residues (WxHxxxxy motif) is crucial for shaping the $\beta$-glucan binding cleft (32). This $\beta$ glucan binding cleft is formed by spatial arrangement of tryptophan, histidine and tyrosine in a triangular fashion resulting in a shallow hydrophobic surface groove, capable of accommodating and binding $\beta$-glucan chains through hydrophobic interactions (31-33). Also true for invertebrates, the same three residues are present in the binding domain of $\beta-(1,3)$-glucan recognition protein (GNBP3), although not as a conserved motif (34). Dectin-1 signalling is activated following clustering in synapse-like structures formed within minutes after activation by particulate $\beta$-glucans (35). Following the interaction with $\beta$-glucan, Dectin-1 signals via Syk kinase and the adaptor protein Card9 to send a downstream signal through Bcl10 and Malt1 to the transcription factor NF- $\mathrm{kB}$ (38). Activation of NF$\kappa B$ leads to an inflammatory profile typical of stimulation with $\beta$-glucans.

The presence or absence in fish genomes of Dectin-1, along with the entire superfamily $V$ of CLRs (NK cell receptors), is debated (42). Although initially a cKLR from Paralabidochromis chilotes was described as a member of the superfamily $V$ (371), and 28 distinct KLR loci identified a particular chromosomal region in Nile tilapia (Oreochromis niloticus) (372), a subsequent and thorough phylogenetic analysis suggested these receptors to be members of superfamily II rather than superfamily $V$ (42). At the same time, it is important to realize that this phylogenetic analysis was primarily based on an early genome assembly of a single fish species, Fugu rubripes (42), indicating that the presence of true superfamily 
V CLR members in fish genomes has not been systematically investigated in other teleosts.

Functional and therefore indirect evidence of the presence of $\beta$-glucan receptor(s) in fish exists for at least Atlantic salmon (Salmo salar) macrophages, channel catfish (Ictalurus punctatus) neutrophils and seabream (Sparus aurata) leukocytes, based on observations that pre-treatment of these cell types with $\beta$ glucans reduces the uptake of yeast (Saccharomyces cerevisiae) glucan particles, zymosan or whole yeast cells (373-375). In carp, injection of $\beta$-glucans induced a complement receptor 3 (CR3)-dependent rosette formation of leukocytes and deposition of iC3b and C3d fragments on zymosan $(130,376)$. $\beta$-glucan was also shown to regulate the expression of several pattern recognition receptors including t/r2 in primary macrophages of European eel (Anguilla anguilla) (377), regulation of tIr3 and $c x c$ receptors in common carp $(111,378)$, the purinergic receptor $p 2 x 4$ in Japanese flounder (Paralichthys olivaceus) (379) and nod 2 and t/r2 in zebrafish (Danio rerio), (175). Although these genes were regulated by $\beta$-glucan stimulation, their involvement in the recognition of $\beta$-glucans remains to be confirmed. Oral intubation of Atlantic salmon (S. salar) with $\beta$-glucan resulted in the up-regulation of syk kinase and three salmon CLRs with one or two ITAMs and a single $\mathrm{WxH}$ motif $(380,381)$, alike the Dectin-1 architecture described above. We reasoned that macrophages could provide an informative starting point for investigating signalling pathways induced upon $\beta$-glucan stimulation. Indeed, primary macrophages of common carp have been shown to respond to curdlan and to zymosan depleted of Toll Like Receptor (TLR) stimulating properties; both considered Dectin-1-specific ligands in mammals (138). We therefore hypothesized that immune-modulatory effects of $\beta$-glucan in carp macrophages could be triggered by an unknown member of the CLR family, different from Dectin-1.

Here, we used primary macrophages of common carp for a whole transcriptome analysis of differentially expressed genes (DEG) induced by two different $\beta$ glucans. Analyses of gene ontology revealed comparable profiles and a clear regulation of the CLR pathway by both $\beta$-glucans. Subsequent investigation of the common carp genome for candidate $\beta$-glucan receptors identified a number of genes based on their architecture or expression profile, all encoding proteins with at least one C-type Lectin Domain (CTLD). Preliminary phylogenetic analysis of the CTLD sequences of candidate proteins showed no clustering with CTLD sequences of known group $V$ members. Synteny analysis of the genome of zebrafish, a close relative of common carp, identified two CTLD-encoding genes with apparent conservation with mammalian CLR group $\mathrm{V}$ members, namely $\mathrm{clec} 4 \mathrm{c}$ and sclra. Overall, our study identifies several teleost CLRs of interest for future functional studies aimed at further specifying the modulatory effects of $\beta$-glucans on the fish immune system. 


\section{Methods}

\section{Animals}

European common carp (Cyprinus carpio carpio L.) of the R3 $\times$ R8 strain were used, which originate from a cross between the Hungarian R8 strain and the Polish R3 strain (169). Carp were bred and raised in the aquatic research facility of Wageningen University, Carus, at $20-23^{\circ} \mathrm{C}$ in recirculating UV-treated water and fed pelleted dry feed (Skretting, Nutreco) twice daily. All experiments were performed with the approval of the animal experiment committee of Wageningen University.

\section{In vitro culture of head kidney-derived macrophages}

Fish were anaesthetized with $0.3 \mathrm{~g} / \mathrm{I}$ Tricaine Methane Sulfonate (TMS) (Crescent Research Chemicals, Phoenix, USA) in aquarium water buffered with $0.6 \mathrm{~g} / \mathrm{l}$ sodium bicarbonate and bled via the caudal vein. Carp head kidney-derived macrophages were cultured for 6 days, as described previously (164), and will be referred to as macrophages.

\section{Macrophage stimulation}

Macrophages were harvested by placing culture flasks on ice for 15 minutes and by gentle scraping. Cell suspensions were centrifuged at $450 \times \mathrm{g}$ for 10 minutes at $4^{\circ} \mathrm{C}$. Macrophages were resuspended in complete NMGFL (incomplete-NMGFL-15 medium supplemented with $2.5 \%$ heat-inactivated pooled carp serum and $5 \%$ bovine calf serum (Invitrogen Life Technologies) with $100 \mathrm{U} / \mathrm{mL}$ of penicillin and $50 \mu \mathrm{g} / \mathrm{mL}$ streptomycin) (164). Subsequently, macrophages were seeded in 24well flat-bottom culture plates (Corning ${ }^{\mathrm{TM}} 3526$, FischerScientific) at $1.5 \times 10^{6}$ macrophages $/ 300 \mu \mathrm{L}$ per well. For stimulation of the cells, curdlan (C7821, Sigma Aldrich) (a high molecular weight linear polymer consisting of $\beta$-1-3-linked glucose residues from Alcaligenes faecalis) and MacroGard ${ }^{\circledR}$ (a cell wall preparation of $S$. cerevisiae comprising $91 \%$-glucan (Zilor, São Paulo, Brazil)) were used (46). $\beta$ glucans were prepared as previously reported (138). Cells were stimulated with $\beta$ glucan preparations at $25 \mu \mathrm{g} / \mathrm{mL}$, a concentration at which both $\beta$-glucan preparations were previously shown to induce considerable nitric oxide production in carp macrophages (138). For each stimulus at least three independent cultures were used and each stimulation was performed in technical triplicate.

After 6 hours of stimulation, three replicate wells were pooled and $4.5 \times 10^{6}$ macrophages were lysed in $350 \mu \mathrm{L}$ RLT buffer (QIAgen, Netherlands) and stored at $-80^{\circ} \mathrm{C}$ until RNA extraction. Total RNA was extracted using the RNeasy Mini kit according to the manufacturer's protocol (QIAgen) including on-column DNase treatment with the RNase-free DNase set (QIAgen). RNA was stored at $-80^{\circ} \mathrm{C}$ until use. 


\section{Illumina sequencing and data analysis}

RNA quality and concentration was checked on a Bioanalyzer (Agilent 2100 total RNA Nano series II chip, Agilent). RNAseq libraries were prepared from $0.5 \mu \mathrm{g}$ total RNA using the TruSeq ${ }^{\circledR}$ Stranded mRNA Library Prep kit according to the manufacturer's instructions (Illumina Inc. San Diego, CA, USA). Similar to the previous carp study (255), all RNAseq libraries were sequenced on an Illumina HiSeq2500 sequencer as $1 \times 50$ nucleotides single-end reads. Image analysis and base calling were performed using the Illumina pipeline. Using TopHat (version 2.0.5), (382), reads were aligned to the latest published genome assembly of common carp (BioProject: PRJNA73579), (255). For each independent sample at least 10 million raw reads were sequenced, on average $65 \%$ of the raw reads could be mapped to annotated genes of this carp genome assembly. Secondary alignments of reads were excluded by filtering the files using SAMtools (version $0.1 .18)$, (383). Aligned fragments per predicted gene were counted from SAM alignment files using the Python package HTSeq (version 0.5.3p9) (384).

Differential gene expression was analysed using the bioinformatics package DESeq 2.0 (v1.22.2), (385) or edgeR (v3.24.3), (386, 387) from Bioconductor (v3.8), (388) in R statistical software (3.1.2), (208). Statistical analysis was performed using a paired design with unstimulated cells as control and performed for curdlan and MacroGard ${ }^{\circledR}$ independently $(n=3$ independent cultures for curdlan and for MacroGard $\left.{ }^{\circledR}\right)$. The paired design allowed for a better comparison between independent cultures, reducing noise generated by culture to culture differences (385). For DESeq 2.0, $p$-values were adjusted using Benjamini \& Hochberg corrections for controlling false discovery rate and results were considered statistically significant when $p$-adjusted $\leq 0.05$. For edgeR, genes were considered significantly regulated if both $p$-value $\leq 0.05$ and FDR $\leq 0.05$. Only genes identified as significantly regulated by both, DESeq 2.0 and edgeR, were used for subsequent analyses (Supplementary tables 1 and 2 for curdlan- and MacroGard ${ }^{\circledR}$-DEGs, respectively). Venn diagrams were generated with the webtool from the University of Ghent Bioinformatics and Evolutionary Genomics group ${ }^{1}$.

\section{Gene Ontology annotation and enrichment analysis}

The common carp genome has been annotated against Ensembl zebrafish GRCz10 (255). Due to its tetraploid nature because of an additional genome duplication event $(253,319,389)$, the common carp generally has two copies of each zebrafish gene. However, for gene ontology (GO) and KEGG analysis only single IDs were used, resulting in a dataset with unique Ensembl zebrafish IDs (curdlan DEGs $n=421$ unique genes and MacroGard ${ }^{\circledR}$ DEGs $n=638$ unique genes). Gene Ontology (GO) analysis was performed with GOrilla (390). Using differentially

${ }^{1}$ http://bioinformatics.psb.ugent.be/webtools/Venn/ 
expressed genes as a target list and the entire list of annotated common carp genes as a background list, GO term enrichment was analysed (Supplementary table 3 and 4 for curdlan and MacroGard ${ }^{\circledR}$, respectively). FDR $q$-values were calculated by adjusting $p$-values using Benjamini \& Hochberg method for controlling false discovery rate, GO terms were considered statistically enriched when FDR $\leq 0.05$.

Independent KEGG analysis (391) was performed using the stable Ensembl zebrafish ID's of each differentially expressed gene in KOBAS v3.0 (392), using the well-annotated zebrafish genome as a reference list. KOBAS was run with Chisquare test and for FDR correction the Benjamini and Yekutieli method was used. Pathways were considered significantly over-represented if the corrected $p$-value was $p \leq 0.05$. Recently, a zebrafish-specific KEGG pathway map for the CLR pathway was released (dre04625). As this map was not yet incorporated in the KOBAS analysis, we performed manual mapping using the "userdata mapping" feature on the zebrafish-specific KEGG pathway for C-type lectin receptor signalling.

\section{Genome search for CTLD-encoding common carp sequences}

The conceptual translation of all annotated carp gene sequences as submitted to NCBI (from here on referred to as carp proteins) (BioProject: PRJNA73579, (255)), was used to perform a Protein family (Pfam) domain search using CLC Main Workbench $v 8.0^{2}$ with the Pfam Database v31 (393). Proteins without a C-type lectin domain (CTLD) (PF00059) were filtered out. Subsequently, using the PatmatDB feature from EMBOSS in the public Galaxy server (v5.0.0) at Wageningen University and Research centre (WUR, The Netherlands ${ }^{3}$ ), protein sequences containing an immunoreceptor tyrosine-based activation motif (ITAM) were identified using the signature $\mathrm{YxxL} / \mathrm{I}$ sequence. Presence of a $\mathrm{WxH}$ motif within the CTLD was investigated using the PatmatDB feature from EMBOSS in Galaxy (v5.0.0) using the signature $\mathrm{WxH}$. Alternatively, presence of a WxHxxxxY motif within the CTLD was investigated using the signature $\mathrm{WxHx}(1,4) \mathrm{Y}$ sequence, allowing for 1 - 4 random residues between histidine and tyrosine. Transmembrane regions of all proteins with at least one CTLD, were predicted using TMHMM Server v. 2.04. These analyses highlighted a restricted number of proteins with one or more CTLDs and characterized the number of ITAM motifs, $\mathrm{W} \times \mathrm{H}$ motifs and transmembrane regions present in the conceptual translations. Subsets of candidate receptors were selected from the restricted number of proteins based on the following three criteria: 1) presence of a conserved $\mathrm{W} \times \mathrm{H}$ motif in the CTLD; 2 ) corresponding expression of $\geq 50$ reads per kilobase million

\footnotetext{
2 https://www.qiagenbioinformatics.com

3 http://galaxy.wur.nl

4 http://www.cbs.dtu.dk/services/TMHMM/
} 
(RPKM) in unstimulated macrophages $(n=5) ; 3)$ differential regulation of expression in carp macrophages stimulated with $\beta$-glucans. Automatic annotation of candidate receptors was manually verified using BLASTx against the $\mathrm{nr}$ database from NCBI.

The CTLD sequences of identified candidate receptors were aligned with CTLD sequences from selected fish CLRs, selected mouse CLRs, several chicken CLRs (394) and with CTLDs from human CTLD-encoding genes (PF00059) present in Ensembl (GRCh38.p12) using MUSCLE v3.8 (395). In case of more than one CTLD sequence per protein, the first CTLD was designated 1 , the next 2 and so on. Subsequently, Model Selection feature of MEGA-X (396) was used to calculate the most appropriate amino acid substitution model using all sites of the alignment as input data. The evolutionary history was inferred by using the Maximum Likelihood (ML) method based on the Whelan and Goldman model (397) allowing for Gamma distribution $(+G)$ with 4 Gamma categories and using the rate variation model allowed for some sites to be evolutionarily invariable $((+\mathrm{I}), 0.19 \%$ sites $)$. The bootstrap consensus tree inferred from $n=500$ replicates was taken to represent the evolutionary history of the taxa analysed (398). Initial tree(s) for the heuristic search were obtained automatically by applying Neighbor-Join and BioNJ algorithms to a matrix of pairwise distances estimated using a JTT model, and then selecting the topology with superior log likelihood value. A discrete Gamma distribution was used to model evolutionary rate differences among sites $(+G$, parameter $=1.6778)$. The final alignment involved a total of $n=223$ CTLD sequences.

As a fourth criterion for the identification of candidate receptors, conservation of synteny with the mammalian NK cell receptor cluster was added. Synteny analysis relied on genomic location data from NCBI and Ensembl Gene Summary databases. The GRCh38.p12, GRCm38.p6 and the Zv10 primary genome assemblies were used for human, mouse, and zebrafish genomic location data, respectively. For genomic location of carp genes the latest common carp assembly (255) was used.

\section{Results}

\section{$\beta$-glucan stimulation of macrophages leads to regulation of the CLR signalling pathway}

Transcriptome analysis of macrophages stimulated with curdlan retrieved a total of $n=528$ differentially expressed genes (DEGs) (Supplementary table 1 ) of which almost $85 \%$ were up-regulated. Transcriptome analysis of macrophages stimulated with MacroGard ${ }^{\circledR}$ retrieved a total of $n=781$ DEGs (Supplementary table 2 ), of which almost $80 \%$ were up-regulated. Subsequent comparison of the expression profile of both DEG datasets revealed a comparable profile, with $n=291$ DEGs that followed concordant expression patterns, only in one case discordant 
regulation was observed as up-regulation by curdlan and down-regulated by MacroGard $^{\circledR}$ (Figure 1).

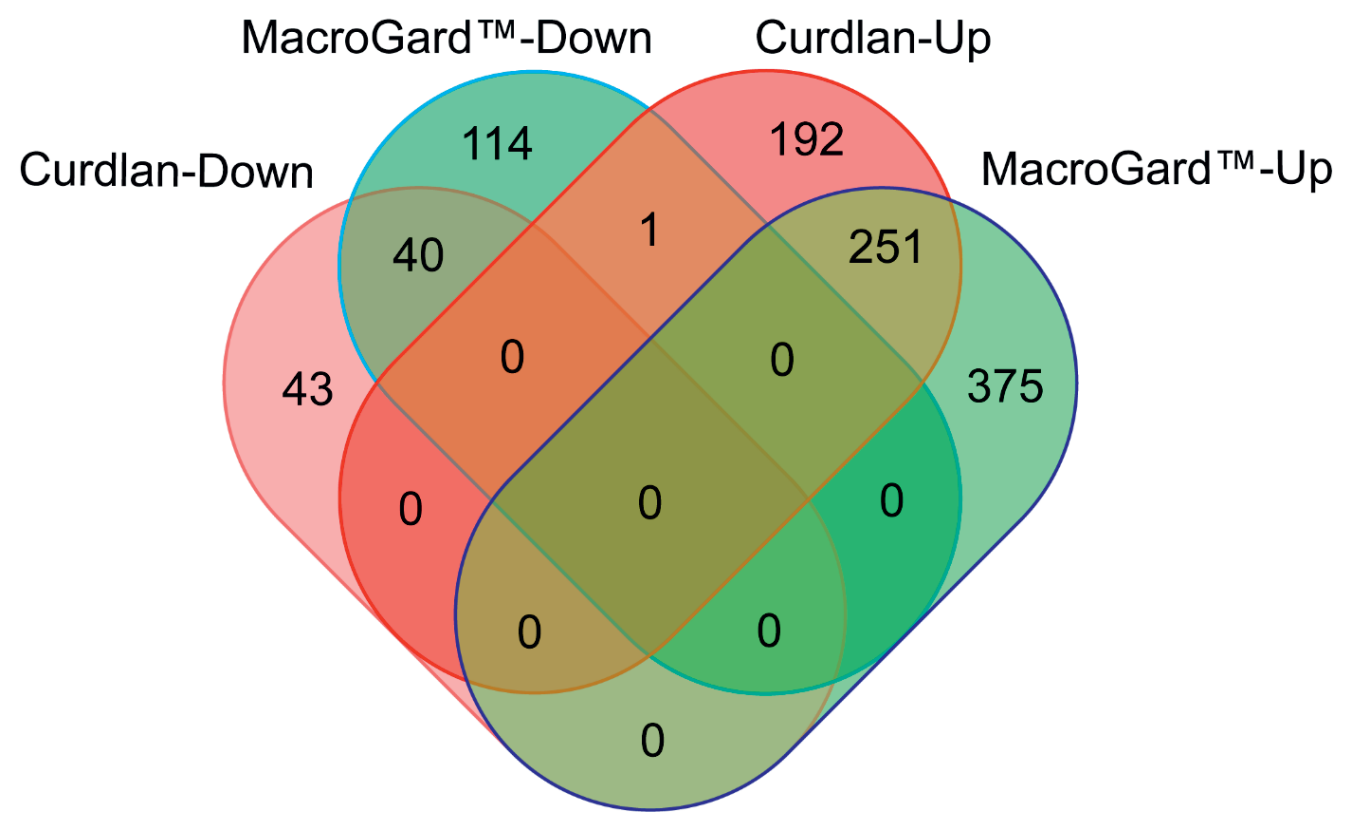

Figure 1. Venn diagram comparing differentially expressed genes (DEGs) regulated upon stimulation of common carp macrophages with $\boldsymbol{\beta}$-glucans. Venn diagram of the DEGs regulated by curdlan (red) or MacroGard ${ }^{\circledR}$ (green). Macrophages were stimulated for 6 hours with $25 \mu \mathrm{g} / \mathrm{mL}$ of either curdlan or MacroGard ${ }^{\circledR}$ and collected for RNAseq analysis.

Automated gene ontology (GO) analysis of the two DEG datasets using Gorilla could map approximately $65 \%$ of the DEGs to GO terms. GO term enrichment analysis revealed 9 and 37 GO terms significantly enriched (FDR $q$-value $\leq 0.05$ ) among curdlan-DEGs and MacroGard $(-D E G s$, respectively. For both datasets, the GO term with smallest FDR $q$-value was "Immune system process (GO:0002376)". Within the domain Biological Process, all significantly enriched GO terms in the curdlan-DEG dataset $(n=7)$, were also significantly enriched in the MacroGard ${ }^{\circledR}$ DEG dataset $(n=25)$ (Supplementary tables 3 and 4 ), suggesting that the gene expression profile regulated by curdlan is also regulated by MacroGard ${ }^{\circledR}$.

Automated annotation of the two DEG datasets to zebrafish KEGG pathways resulted in the mapping of the curdlan-DEGs to $n=92$ different pathways and of the MacroGard ${ }^{\circledR}$-DEGs to $n=112$ different pathways. Four pathways were significantly over-represented in both DEG datasets, and a further nine unique pathways were significantly over-represented only in the MacroGard ${ }^{\circledR}$-DEGs (Table 1). The overlap in the over-represented KEGG pathways in the two datasets supports the notion that in carp macrophages, the profile induced by curdlan is also induced by MacroGard ${ }^{\circledR}$. 
Table 1: Over-represented KEGG pathways following automated KEGG analysis of differentially expressed genes (DEGs) in macrophages stimulated with curdlan or MacroGard ${ }^{\circledR}$. Curdlan-DEGs ( $n=356$ genes) could be mapped to $n=92$ pathways, of which $n=4$ were significantly over-represented. MacroGard ${ }^{\circledR}$-DEGS ( $n=541$ genes) could be mapped to $n=112$ pathways of which $n=13$ were significantly over-represented. KEGG analysis was performed with KOBAS v3.0 with Chi-square test and the Benjamini and Yekutieli method for FDR correction. Pathways were considered significantly overrepresented at corrected $\mathrm{p}$-value $\leq 0.05$.

\begin{tabular}{|l|c|c|}
\hline Pathway & & $\mathbf{X}$ \\
\hline Phagosome & $\mathbf{X}$ & $\mathbf{X}$ \\
\hline Cytokine-cytokine receptor interaction & & $\mathbf{X}$ \\
\hline Lysosome & $\mathbf{X}$ & $\mathbf{X}$ \\
\hline Apoptosis & & $\mathbf{X}$ \\
\hline Herpes simplex infection & & $\mathbf{X}$ \\
\hline Toll-like receptor signalling pathway & $\mathbf{X}$ & $\mathbf{X}$ \\
\hline NOD-like receptor signalling pathway & & $\mathbf{X}$ \\
\hline VEGF signalling pathway & & $\mathbf{X}$ \\
\hline Arachidonic acid metabolism & & $\mathbf{X}$ \\
\hline Metabolic pathways & & $\mathbf{X}$ \\
\hline Phosphatidylinositol signalling system & $\mathbf{X}$ & $\mathbf{X}$ \\
\hline ECM-receptor interaction & & $\mathbf{X}$ \\
\hline Adipocytokine signalling pathway & & \\
\hline
\end{tabular}

Automated annotation did not include the zebrafish-specific KEGG map for the "Ctype lectin receptor signalling pathway" (dre04625) which has become available only recently. Manual mapping of curdlan- and MacroGard ${ }^{\circledR}$-DEGs showed regulation of a large number of genes associated with the C-type lectin receptor pathway (Figure 2). Further, not only end-products of the CLR signalling pathway, such as cytokines which are often shared among pathways, but also several upstream molecules such as card9, bcl10, malt1 and calm were regulated (see Figure 2). The manual mapping suggests that in carp macrophages, both $\beta$-glucans induced expression of genes typically associated with C-type lectin receptor signalling. 


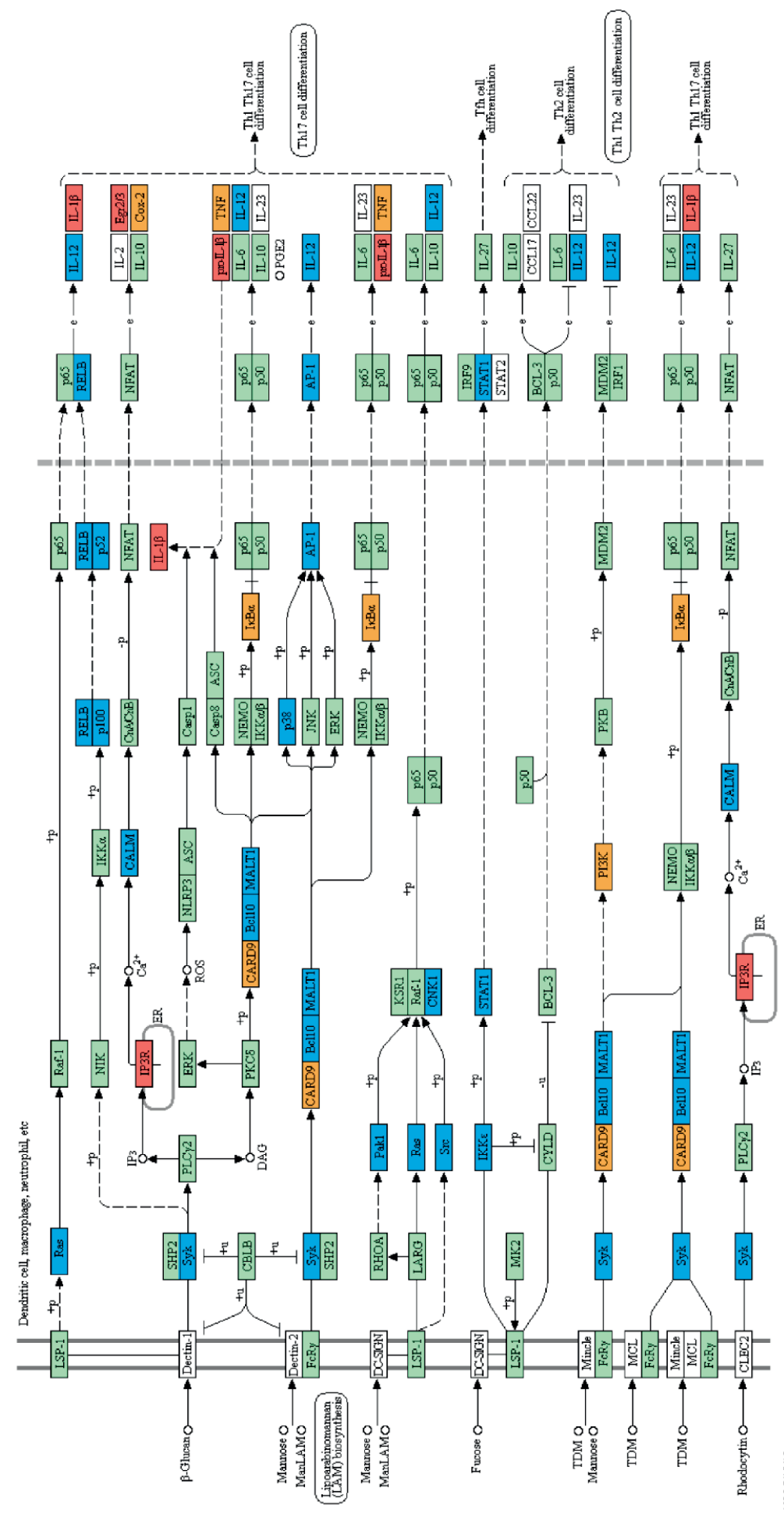

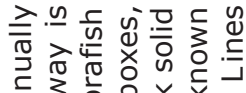

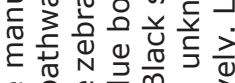

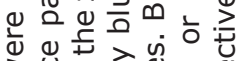

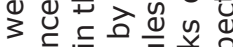

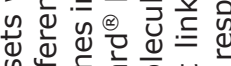

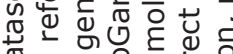

西 $\frac{0}{0}$ 元

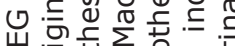

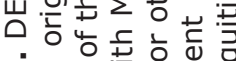

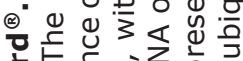

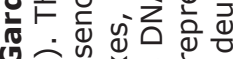

ए人i

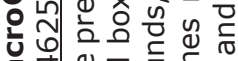

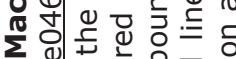

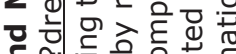

당

달

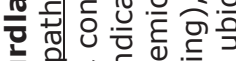

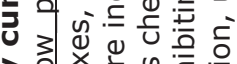

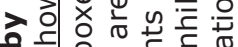

जी 0

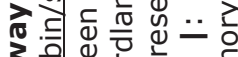

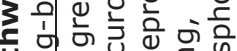

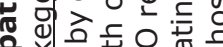

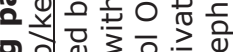

읭

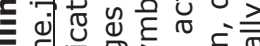

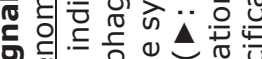

흔

길

约

의의

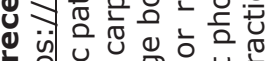

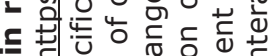

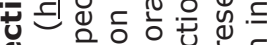
는 은응

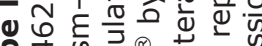
웅.

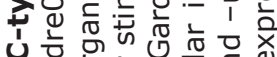

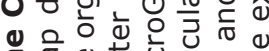

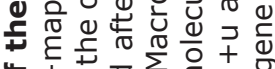
¿ <

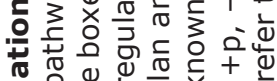
죽

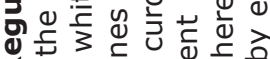

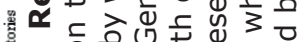

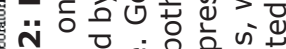

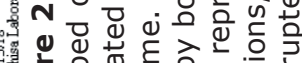
臂

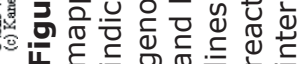




\section{Search for candidate receptors}

Following the above-described mapping of DEGs to the CLR pathway, we continued with screening all 50,527 conceptually translated carp proteins for the presence of at least one CTL domain (CTLD), narrowing down the search for candidate receptors to a total of $n=239$ proteins. These CTLD-containing proteins were further characterized for the presence of ITAM sequences and transmembrane helices (data not shown). Studies have shown that the presence of a $\mathrm{WxH}$ motif or a WxHxxxxY motif in the carbohydrate binding region of Dectin-1 determines the $\beta$-glucan binding capacities of Dectin-1 (31-33). This criterion was used to further narrow down the search for candidate receptors, identifying a subset of $n=13$ carp proteins with a $\mathrm{WxH}$ motif (Table 2 ). We could not identify proteins with a $\mathrm{W} x \mathrm{H} x \mathrm{xxxY}$ motif present specifically in their CTLD. Interestingly, in four $\mathrm{WxH}-$ containing proteins, we could also identify a transmembrane region and one or two ITAMs and thus a 'complete' Dectin-1-like architecture. However, based on the RNAseq analysis, none of these candidates was significantly regulated by $\beta$-glucan stimulation or were constitutively expressed (higher than 10 reads per kilobase million) in unstimulated macrophages (Table 2), suggesting that these 13 candidates could not likely explain the functional responses to $\beta$-glucans in our current experimental set up.

Table 2: Candidate receptors with a $\mathrm{WxH}$ signature sequence in their CTL domain. Description refers to the closest BLASTx hit upon re-blasting the identified genes of interest and accession numbers refer to protein sequences in NCBI. Number of conserved CTL domains (CTLDs), ITAM sequences and transmembrane domains (TM) in the protein sequence are included. RPKM refers to the average $(n=5)$ reads per kilobase million in unstimulated macrophages and is a measure for gene expression. cypCar codes identify common carp genes (Cyprinus carpio). Names or numbers in brackets refer to genes with identical BLASTx hits (likely owing to the additional genome duplication event in common carp). Proteins with architecture similar to Dectin-1 are highlighted in grey. Candidate receptors are ordered numerically by cypCar code.

\begin{tabular}{|c|c|c|c|c|c|c|}
\hline Description & Accession number & CTLD & ITAM & TM & RPKM & cypCar \\
\hline $\begin{array}{l}\text { CD248 molecule, } \\
\text { endosialin A } \\
\text { precursor }\end{array}$ & NP_001092698.3 & 1 & 2 & 1 & 0.00 & 00001997 \\
\hline $\begin{array}{l}\text { C type lectin } \\
\text { receptor A }\end{array}$ & NP_001117051.1 & 1 & $\begin{array}{l}3 \\
(4)\end{array}$ & 1 & $0.45(0.00)$ & $\begin{array}{l}00016746, \\
(00029396)\end{array}$ \\
\hline Aggrecan & BAJ61837.1 & 1 & 3 & 0 & 0.00 & 00023554 \\
\hline $\begin{array}{l}\text { Novel protein with } \\
\text { Lectin C-type } \\
\text { domains precursor }\end{array}$ & NP_001093528.1 & 3 & 2 & 0 & 0.01 & 00031652 \\
\hline $\begin{array}{l}\text { Novel protein } \\
\text { similar to lectins }\end{array}$ & CAI21223.1 & $\begin{array}{l}1 \\
(2)\end{array}$ & 1 & 0 & 0.00 & $\begin{array}{l}00037242, \\
(00037243)\end{array}$ \\
\hline Collectin 12 & BAU33575.1 & 1 & 2 & 1 & 0.12 & 00040460 \\
\hline $\begin{array}{l}\text { C-type mannose } \\
\text { receptor 2-like }\end{array}$ & XP_026052210.1 & $\begin{array}{l}3 \\
(1)\end{array}$ & $\begin{array}{l}1 \\
(4)\end{array}$ & 0 & $0.02(0.00)$ & $\begin{array}{l}00041550, \\
(00047187)\end{array}$ \\
\hline $\begin{array}{l}\text { Secretory } \\
\text { phospholipase A2 } \\
\text { receptor-like }\end{array}$ & XP_026074237.1 & 2 & 1 & 0 & 0.00 & 00045110 \\
\hline C-type lectin 1 & AEH76769.1 & 1 & 1 & 1 & 6.67 & 00046286 \\
\hline $\begin{array}{l}\text { Macrophage } \\
\text { mannose receptor } \\
\text { 1-like }\end{array}$ & XP_026074235.1 & 2 & 1 & 1 & 0.00 & 00048442 \\
\hline
\end{tabular}


As the above-mentioned screening for $\mathrm{WxH}$ motif did not identify candidate receptors expressed or regulated in carp macrophages, we widened again our search and used constitutive expression or differential regulation as new criteria to narrow down the search for candidate receptors. Screening of all $n=239$ CTLDcontaining proteins for constitutive expression of their corresponding gene identified a subset of $n=12$ genes that were expressed at an arbitrary threshold set at $>50$ Reads Per Kilobase Million (RPKM), corresponding to on average $1.5 \%$ of $\beta$-actin expression. Screening for regulation identified a subset of $n=6$ candidate receptors as differentially expressed after stimulation with $\beta$-glucans, all of which overlapped with the expressed subset (Table 3 ). Of interest, all six were downregulated by $\beta$-glucan stimulation and two out of the six candidate receptors (both Asialoglycoprotein receptor orthologues) were regulated by both $\beta$-glucans. The finding that these candidate receptors were all regulated could suggest involvement in the response to $\beta$-glucan simulation of carp macrophages.

Table 3: Candidate receptors expressed in carp macrophages. Candidates were selected based on a minimal expression of $\geq 50$ RPKM in unstimulated carp macrophages or differential expression following stimulation of macrophages with $\beta$-glucans. Description refers to the closest BLASTx hit, with associated NCBI protein accession code. Number of conserved CTL domains (CTLDs), ITAM sequences and transmembrane domains (TM) in the protein sequence are included. RPKM refers to the average $(n=5)$ reads per kilobase million in unstimulated macrophages and is a measure for gene expression. CRD and MG refer to differentially expressed genes in macrophages after stimulation with curdlan (CRD) or MacroGard ${ }^{\circledR}$ (MG) based on RNAseq analysis (highlighted in grey). cypCar codes identify common carp genes (Cyprinus carpio). Names or numbers in brackets refer to genes with identical BLASTx hits (duplicated genes). Candidate proteins are ordered based on their expression (RPKM).

\begin{tabular}{|c|c|c|c|c|c|c|c|c|}
\hline Description & $\begin{array}{l}\text { Accession } \\
\text { number }\end{array}$ & CTLD & ITAM & TM & RPKM & CRD & MG & cypCar \\
\hline zgc:174904 & NP_001170922.1 & 1 & 0 & 1 & 836 & & $x$ & 00031274 \\
\hline $\begin{array}{l}\text { Asialoglycoprotein } \\
\text { receptor } 1 \text {-like } \\
\text { isoform } 1\end{array}$ & XP_026096377.1 & 2 & 1 & 1 & 654 & $x$ & $x$ & 00032252 \\
\hline $\begin{array}{l}\text { Mannose receptor c } \\
\text { type } 1\end{array}$ & ALS87701.1 & 2 & $\begin{array}{l}2 \\
(6)\end{array}$ & 0 & $\begin{array}{l}467 \\
(354)\end{array}$ & & & $\begin{array}{l}00046879 \\
(00034006)\end{array}$ \\
\hline $\begin{array}{l}\text { Asialoglycoprotein } \\
\text { receptor } 1 \text {-like } \\
\text { isoform } \times 2\end{array}$ & XP_026098956.1 & 1 & 0 & 1 & 239 & $\mathrm{x}$ & $\mathrm{x}$ & 00032253 \\
\hline Ladderlectin-like & XP 026129733.1 & 1 & 0 & 0 & 215 & & & 00046054 \\
\hline $\begin{array}{l}\text { C-type lectin } \\
\text { domain family } 4 \\
\text { member E-like }\end{array}$ & XP_026087308.1 & 1 & 0 & 1 & 200 & & & 00044344 \\
\hline $\begin{array}{l}\text { C-type lectin } \\
\text { domain family } 4 \\
\text { member C }\end{array}$ & NP_001313501.1 & 1 & 1 & 1 & 165 & & $\mathrm{x}$ & 00024225 \\
\hline $\begin{array}{l}\text { Macrophage } \\
\text { mannose receptor } \\
\text { 1-like }\end{array}$ & XP_026142830.1 & $\begin{array}{l}2 \\
(5)\end{array}$ & $\begin{array}{l}1 \\
(7)\end{array}$ & 1 & $\begin{array}{l}92 \\
(60)\end{array}$ & & $\mathrm{x}$ & $\begin{array}{l}00047225, \\
(00023855)\end{array}$ \\
\hline CD209 antigen & XP_003197805.3 & 1 & 0 & 0 & 83 & & & 00049168 \\
\hline $\begin{array}{l}\text { C-type lectin } \\
\text { domain family } 4 \\
\text { member E }\end{array}$ & XP_002660626.3 & 1 & 0 & 0 & 60 & & & 00014657 \\
\hline
\end{tabular}




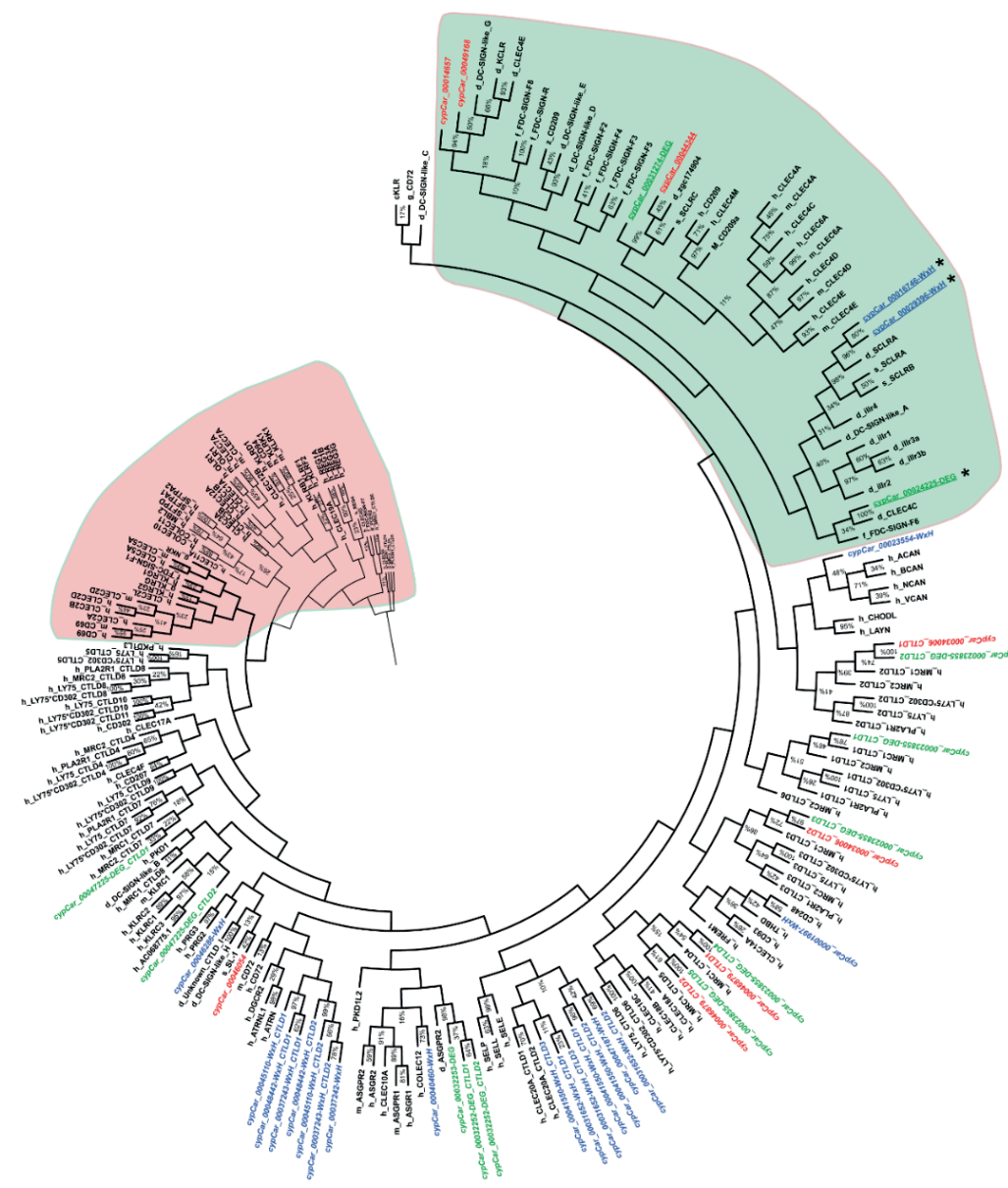

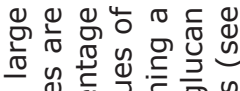

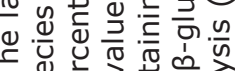

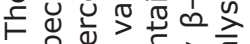

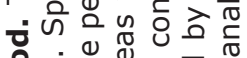

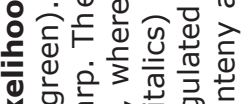

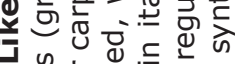

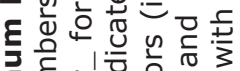

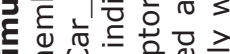

X

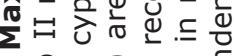

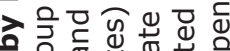

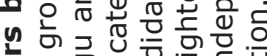

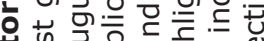

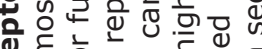

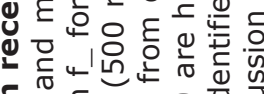

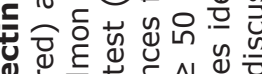

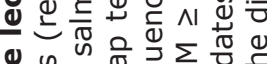

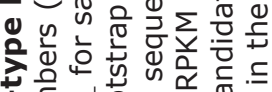

ปُ

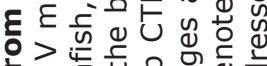

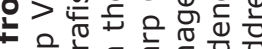

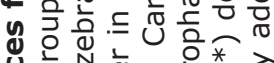

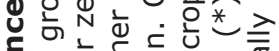

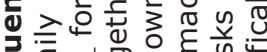

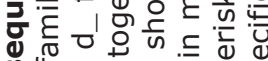

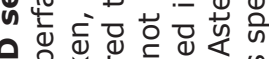

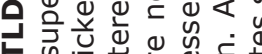

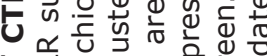

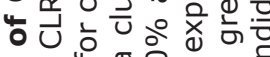

는

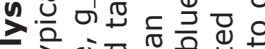

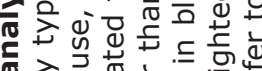

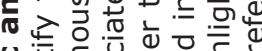

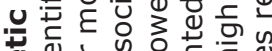

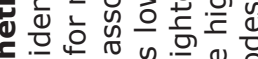

둔

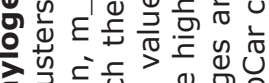

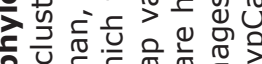

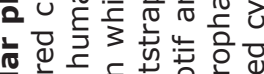

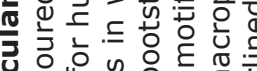

उु 응 원

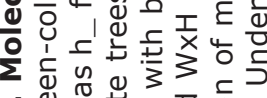

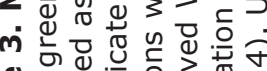

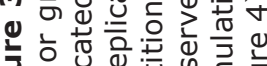

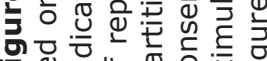

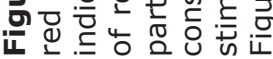


Combining the subsets of candidate $\beta$-glucan receptors identified based on criteria 1 (WxH motif), 2 (RPKM $\geq 50$ ) and 3 (differential regulation) resulted in a total of $n=25$ proteins of specific interest, containing $n=39$ CTLD sequences. To investigate the phylogenetic relation between these candidates of interest and known CLR family members, CTLD sequences were aligned. The phylogenetic tree revealed a clear subdivision of the cypCars related to the carp CTLD proteins. None of the CTLD sequences from the identified candidates clustered with family $\mathrm{V}$ members, the group containing Dectin-1 (Figure 3). Instead, seven CTLD sequences from $\mathrm{W} \times \mathrm{H}$ motif-containing candidates (criterion 1) clustered with human CLEC20A, among human CLR group II members, and seven other $\mathrm{WxH}$-candidates clustered together with human Attractin. Maybe not surprisingly, four of the constitutively expressed CTLD sequences (criterion 2) clustered with CTLD sequences from macrophage mannose receptors, group VI family. Six CTLD sequences from the differentially regulated subset (criterion 3) also clustered with macrophage mannose receptors, the other six CTLD sequences in this subset followed no specific clustering pattern. Overall, preliminary phylogenetic analysis suggests the absence of group $\mathrm{V}$ members in our set of candidate receptors.

\section{Synteny analysis of zebrafish and carp CTLD-encoding genes}

Investigation of the carp CTLD containing proteins identified several candidates with architecture similar to mammalian Dectin-1. In mammalian genomes Dectin1 is located in the NK cell receptor cluster, which contains several CLRs. This NK cell receptor cluster shows conserved synteny between human chromosome 12 (hCHR12) and mouse chromosome 6 (mCHR6) (399). Conservation of synteny describes preservation of co-localization of genes on chromosomes of different species and might therefore serve as an additional criterion (criterion 4) for the identification for candidate receptors involved in the recognition of $\beta$-glucans. Synteny analysis can be best performed in a well-assembled genome. The genome assembly of zebrafish, a close relative of common carp, is among the best assembled genomes in teleost fish, including large chromosome scaffolds that can be used for synteny analysis, in contrast to the carp genome which is still largely fragmented into small scaffolds $(253,255)$. The NK cell receptor cluster in human includes, among others, DECTIN-1, DECTIN-2, and MINCLE. Regions surrounding the NK cell receptor cluster on hCHR12 and mCHR6 showed conserved synteny with regions on zebrafish chromosome 16 (zCHR16), based on co-localization of pex5, clstn3, Ipcat3, gnb3, cops7a, and znf384 (Figure 4). Intriguingly, this region of zCHR16 also includes two CTLD-encoding genes, clec4C (NCBI Gene ID: 563797) and sclra (NCBI Gene ID: 564061), highlighting them both as genes of interest for our study.

Further investigation of synteny between the zebrafish region of interest on zCHR16 and carp scaffolds revealed partial conservation of synteny with four scaffolds. Two of these scaffolds contained cypCar_00024225 and cypCar_00032523, putative paralogues of zebrafish clec4c; of which cypCar_00024225 is a CTLD-encoding gene, already identified in this study as 


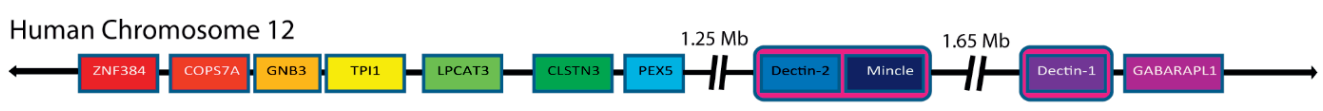

Zebrafish Chromosome 16

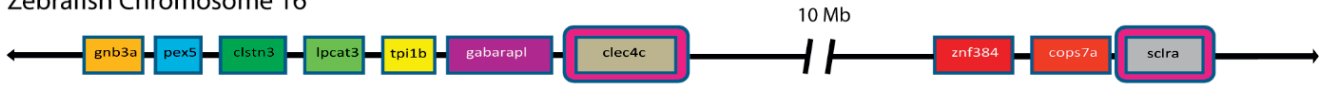

Mouse Chromosome 6

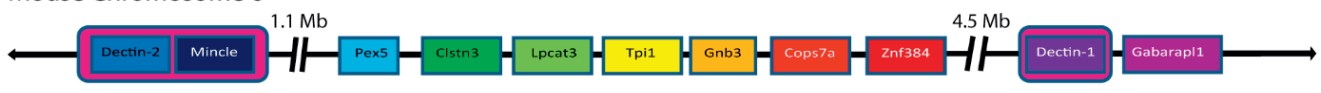

Carp scaffold \#39981

size: $\sim 79 \mathrm{~kb}$

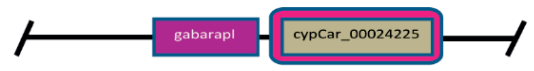

Carp scaffold \#21540

size: $\sim 51 \mathrm{~kb}$

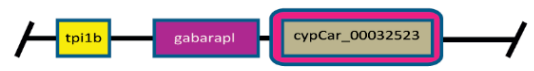

Carp scaffold \#30078

size: $\sim 112 \mathrm{~kb}$

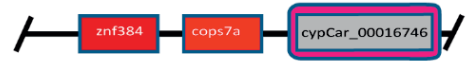

Carp scaffold \#1727

size: $\sim 61 \mathrm{~kb}$

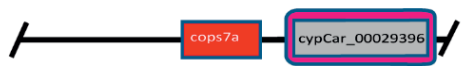

Figure 4. Synteny analysis of mammalian CLR group $V$ cluster on human chromosome 12 and mouse chromosome 6 shows partial conservation with zebrafish chromosome 16 and four carp scaffolds. Partial conservation of synteny between zebrafish chromosome 16 and the mammalian CLR group V cluster can be observed. Among the syntenic genes, two zebrafish CLTD-encoding genes are located, clec4c (NCBI Gene ID: 563797) and sclra (NCBI Gene ID: 564061). Synteny of zebrafish clec4c and sclra with Dectin-1, Dectin-2, and Mincle is highlighted by pink boxes. Synteny of carp cypCars is based on conservation of genes surrounding a CTLD-encoding gene for both clec4c and scrla. Large gaps between loci are indicated with breaks and chromosomal representations are not drawn to scale. Owing to the tetraploid nature of carp, two corresponding scaffolds for each zebrafish gene are shown.

candidate receptor based on its regulation in macrophages after stimulation with MacroGard ${ }^{\circledR}$ (criterion 3). The two other scaffolds contained cypCar_00016746 and cypCar_00029396, putative paralogues of zebrafish sclra; both of which are CTLDencoding carp genes, already identified in this study as candidate receptors based on their conserved $\mathrm{WxH}$-motif (criterion 1 ). The synteny analysis provided a fourth criterion, additional to the previously formulated criteria ( $\mathrm{W} \times \mathrm{H}$ motif, constitutive expression and differential regulation), to identify candidate receptors for $\beta$-glucan recognition. A Venn diagram, graphically representing the different subsets of candidate $\beta$-glucan receptors identified by the four criteria can be found Supplementary figure 1 . 


\section{Discussion}

Primary macrophages of common carp had previously been shown to respond to prototypical Dectin-1 ligands, which led to the hypothesis that the CLR pathway must play an important role in the recognition of $\beta$-glucans in carp macrophages. In our approach, we used head kidney-derived carp macrophages as a starting cell population to test our hypothesis and investigate activation of the CLR pathway upon stimulation with $\beta$-glucans. Indeed, pathway analysis of differentially expressed genes confirmed our hypothesis that $\beta$-glucans regulate a downstream signalling pathway typical of CLR activation. Further, we could identify in the transcriptome of $\beta$-glucan-stimulated carp macrophages, several differentially expressed genes with a C-type lectin domain. These data are of high interest for further functional studies on the mechanisms underlying $\beta$-glucan-induced immunomodulation in teleost fish.

We used two different $\beta$-glucans: curdlan, a linear polymer of $\beta$-(1,3)-linked glucose and considered a Dectin-1-specific ligand, and MacroGard ${ }^{\circledR}$, a branched polymer of $\beta$-(1,3/1,6)-glucose widely-applied as feed additive in aquaculture. Overall, MacroGard ${ }^{\circledR}$ regulated a higher number of differentially expressed genes than curdlan, possibly owing to differences in purity, source, degree of polymerization, and nature of the glycosidic bonds in the $\beta$-glucans (23). Regardless of the extent of gene regulation, manual mapping of the DEGs revealed a clear regulation of the CLR signalling pathway (KEGG) for both $\beta$-glucans. Indeed, up-regulation of homologues of all three players of the card9-Bcl10-Malt1 complex, previously shown to play a crucial role in $\beta$-glucan signalling through the CLR pathway (38), strongly supports regulation by the CLR pathway. We continued our study by identifying candidate genes encoding for proteins with one or more C-type Lectin Domains (CTLD), which could be of potential interest with respect to recognition of $\beta$-glucans, using a recently published database of RNAseq-validated gene predictions for carp (255). We used four criteria to identify candidate receptors in carp macrophages: 1 ) conservation of the glucan binding $\mathrm{WxH}$-motif in the CTLD; 2) constitutive expression higher than 50 RPKM; 3) differential regulation upon stimulation with $\beta$-glucans; 4) conservation of synteny with mammalian NK cell receptor cluster.

Based on criterion 1 (conserved $\mathrm{W} \times \mathrm{H}$ motif) we identified two candidates (cypCar_00016746 and cypCar_00029396) of which the CTLD clustered together with known CTLD sequences from zebrafish and from Atlantic salmon, known as salmon C-type lectins sclra and sclrb (381). Although the carp sclr paralogues were not constitutively expressed in macrophages (criterion 2), both salmon sclrs have been associated with the response to $\beta$-glucans (380). Interestingly, the mammalian $\mathrm{WxHx}$ [4] $\mathrm{Y}$ motif was not conserved, however a motif with five rather than four residues $(\mathrm{W} \times \mathrm{H} \times[5] \mathrm{Y})$ separating histidine from tyrosine was conserved between all sequences. All three residues are considered crucial to form the $\beta$ glucan binding cleft of mammalian Dectin-1 (32), and also present in invertebrate $\beta$-glucan binding proteins (GNBP3), but not as a $\mathrm{WxHx}[5] \mathrm{Y}$ motif $(32,34)$. 
Although not constitutively expressed in macrophages, carp sclr could well play a role in $\beta$-glucan binding in other cell types.

Based on criterion 2 (constitutive expression $\geq 50$ RKPM), we identified a further 13 candidate receptors, of which six were differentially regulated (criterion 3 ). Without exception, all six genes were down-regulated upon stimulation with $\beta$ glucans, which could possibly be explained by a need to restrict de novo protein synthesis and duration of signalling to prevent over-stimulation (400-403). Possibly, analysis of protein and/or gene expression at different time points could show up-regulation. In Atlantic salmon, three sclrs were up-regulated seven days after oral administration of MacroGard ${ }^{\circledR}$, concomitantly with syk kinase (380). We similarly noticed a concomitant regulation of $s y k$, suggesting the expression of CTLD-encoding genes and syk is co-regulated. No matter what, the observed modulation of gene expression strongly suggests involvement of CLR family members upon recognition of $\beta$-glucans by carp macrophages.

Based on criterion 3 (regulation of gene expression), we identified several additional candidate receptors. Among these, the CTLD-encoding gene with the highest expression and regulation in carp macrophages (cypCar_00031274), clustered together with three other genes of interest: i) a CTLD-encoding gene already identified in this study based on criterion 2 (cypCar_00044344), ii) an unknown zebrafish full length cDNA (zgc174904) encoding a protein with a CTLD, a transmembrane helix and a $\mathrm{WxHx}[5] \mathrm{Y}$ motif just outside the borders of the CTLD and iii) an Atlantic salmon C-type lectin receptor-c (sclrc), different from the ones previously mentioned in criterion 1 . The salmon sclr genes were first identified in (suppression subtractive) EST libraries (381), while a follow up study revealed upregulation of all three sclr genes after oral intubation of Atlantic salmon with MacroGard $^{\circledR}$ (380). The salmon sclrc gene contains a WxH motif within the CTLD while the carp candidate genes (both, cypCar_00031274 and cypCar_00044344)

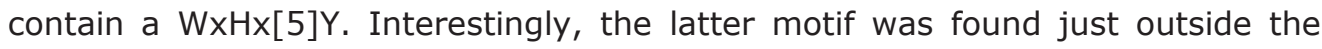
boundaries predicted for a CTLD, which suggests that further manual scrutiny of predicted domains in fish sequences could expand the list of currently identified domains of interest. Overall, the fact that closely-related sc/r genes were identified as candidate receptors in both, salmon and carp, suggests a potential role of this CTL receptor in the response to $\beta$-glucans in fish and supports a need for its further characterization of function.

Based on criterion 4 (synteny in the zebrafish genome), we identified two CTLDencoding genes of interest; putatively named clec4c and sclra. Three out of four corresponding duplicates at syntenic regions of the carp genome were already identified as genes of interest based on the selection criteria discussed above. This means that next to the sclr genes, we could identify clec4c as another gene of interest deserving further attention as candidate receptor for $\beta$-glucan. Taken together, our broad NGS approach helped us describe a clear regulation of the CLR pathway and identify a number of CTLD-containing candidate receptors for $\beta$ glucan binding. As proteins, these receptors would form a good starting point for 
future sugar binding assays and for further functional characterization with e.g. glycome microarrays, a high-throughput method devised to analyse $\beta$-glucanbinding proteins through an oligosaccharide microarray, followed by massspectrometric sequencing $(404,405)$. Altogether, the candidates discussed in this study should help pave the way to future functional studies that could ultimately lead to the identification of $\beta$-glucan receptor(s) in fish.

\section{Author Contributions}

JP, MF and GW contributed to the design of the experiments, acquisition of samples, and analysis of data. GW acquired funding. RW and EC contributed with the phylogenetic and synteny analysis. $\mathrm{CO}$ contributed with reagents, materials and analysis tools. JP, RW, MF and GW wrote the manuscript.

\section{Funding}

This research was funded by the Netherlands Organisation for Scientific Research and São Paulo Research Foundation, Brazil (FAPESP) as part of the Joint Research Projects BioBased Economy NWO-FAPESP Programme (Project number 729.004.002). EB was supported by fellowships from the Maine INBRE Program through NIH grant P20GM103423. RW is a Burroughs Wellcome Fund Investigator in the Pathogenesis of Infectious Disease and this work was supported by NIH grant R15AI133415.

\section{Acknowledgements}

Pierre Boudinot is gratefully acknowledged for his suggestions on the phylogenetic analysis investigating the evolutionary relationships between CTLD sequences. 


\section{Supplementary material}

Supplementary tables 1 and 2 are excluded due to the size of the tables. The tables can be accessed at

https://www.frontiersin.org/articles/10.3389/fimmu.2019.00280/full.

Supplementary table 3. GOrilla GO enrichment analysis of curdlan differentially expressed genes (DEGs). False discovery rate (FDR) correction is performed using the Benjamini and Hochberg (1995) method.

\begin{tabular}{llllc}
\hline Domain & GO Term & Description & FDR & \# DEG \\
& & q-value & \\
\hline GO molecular function & GO:0032813 & $\begin{array}{l}\text { tumor necrosis factor } \\
\text { receptor superfamily binding }\end{array}$ & $1.99 \mathrm{E}-02$ & 6 \\
GO molecular function & GO:0005164 & $\begin{array}{l}\text { tumor necrosis factor } \\
\text { receptor binding }\end{array}$ & $9.94 \mathrm{E}-03$ & 6 \\
\hline GO Biological Process & GO:0002376 & immune system process & $2.02 \mathrm{E}-03$ & 22 \\
GO Biological Process & GO:0043207 & $\begin{array}{l}\text { response to external biotic } \\
\text { stimulus }\end{array}$ & $4.39 \mathrm{E}-03$ & 15 \\
GO Biological Process & GO:0009607 & response to biotic stimulus & $3.22 \mathrm{E}-03$ & 15 \\
GO Biological Process & GO:0006955 & immune response & $7.22 \mathrm{E}-03$ & 15 \\
GO Biological Process & GO:0006952 & defense response & $1.19 \mathrm{E}-02$ & 14 \\
GO Biological Process & GO:0070309 & lens fiber cell morphogenesis & $2.07 \mathrm{E}-02$ & 3 \\
GO Biological Process & GO:0006954 & inflammatory response & $4.28 \mathrm{E}-02$ & 9 \\
\hline
\end{tabular}


Supplementary table 4. GOrilla GO enrichment analysis of MacroGard ${ }^{\circledR}$ differentially expressed genes (DEGs). False discovery rate (FDR) correction is performed using the Benjamini and Hochberg (1995) method.

\begin{tabular}{|c|c|c|c|c|}
\hline Domain & GO Term & Description & $\begin{array}{l}\text { FDR q- } \\
\text { value }\end{array}$ & \# DEG \\
\hline GO Molecular Function & GO:0005126 & cytokine receptor binding & $4.12 \mathrm{E}-03$ & 15 \\
\hline GO Molecular Function & GO:0004896 & cytokine receptor activity & $3.48 \mathrm{E}-03$ & 11 \\
\hline GO Molecular Function & GO:0005164 & $\begin{array}{l}\text { tumor necrosis factor } \\
\text { receptor binding }\end{array}$ & 4.97E-02 & 6 \\
\hline GO Molecular Function & GO:0046961 & $\begin{array}{l}\text { proton-transporting ATPase } \\
\text { activity, rotational } \\
\text { mechanism }\end{array}$ & $3.98 \mathrm{E}-02$ & 6 \\
\hline GO Molecular Function & GO:0060589 & $\begin{array}{l}\text { nucleoside-triphosphatase } \\
\text { regulator activity }\end{array}$ & 4.79E-02 & 16 \\
\hline GO Molecular Function & GO:0042625 & $\begin{array}{l}\text { ATPase coupled ion } \\
\text { transmembrane transporter } \\
\text { activity }\end{array}$ & 4.67E-02 & 9 \\
\hline GO Molecular Function & GO:0019829 & $\begin{array}{l}\text { cation-transporting ATPase } \\
\text { activity }\end{array}$ & $4.21 \mathrm{E}-02$ & 9 \\
\hline GO Molecular Function & GO:0098772 & molecular function regulator & $4.41 \mathrm{E}-02$ & 48 \\
\hline GO Molecular Function & GO:0005096 & GTPase activator activity & $4.88 \mathrm{E}-02$ & 14 \\
\hline GO Biological Process & GO:0002376 & immune system process & $8.35 \mathrm{E}-08$ & 35 \\
\hline GO Biological Process & GO:0006952 & defense response & $1.31 \mathrm{E}-07$ & 25 \\
\hline GO Biological Process & GO:0006955 & immune response & $4.38 \mathrm{E}-07$ & 25 \\
\hline GO Biological Process & GO:0043207 & $\begin{array}{l}\text { response to external biotic } \\
\text { stimulus }\end{array}$ & $1.45 \mathrm{E}-06$ & 23 \\
\hline GO Biological Process & GO:0009607 & response to biotic stimulus & $1.35 \mathrm{E}-06$ & 23 \\
\hline GO Biological Process & GO:0051707 & response to other organism & 2.95E-05 & 19 \\
\hline GO Biological Process & GO:0050896 & response to stimulus & $1.16 \mathrm{E}-04$ & 78 \\
\hline GO Biological Process & GO:0051704 & multi-organism process & $1.10 \mathrm{E}-04$ & 19 \\
\hline GO Biological Process & GO:0007165 & signal transduction & $2.69 E-04$ & 101 \\
\hline GO Biological Process & GO:0006954 & inflammatory response & $7.08 \mathrm{E}-04$ & 13 \\
\hline GO Biological Process & GO:0009605 & response to external stimulus & $5.60 \mathrm{E}-03$ & 26 \\
\hline GO Biological Process & GO:0098542 & $\begin{array}{l}\text { defense response to other } \\
\text { organism }\end{array}$ & $6.04 \mathrm{E}-03$ & 11 \\
\hline GO Biological Process & GO:0009617 & response to bacterium & $6.62 \mathrm{E}-03$ & 11 \\
\hline
\end{tabular}




\begin{tabular}{|c|c|c|c|c|}
\hline GO Biological Process & GO:0006935 & chemotaxis & $7.28 \mathrm{E}-03$ & 13 \\
\hline GO Biological Process & GO:0060326 & cell chemotaxis & $1.09 \mathrm{E}-02$ & 10 \\
\hline GO Biological Process & GO:0019221 & $\begin{array}{l}\text { cytokine-mediated signaling } \\
\text { pathway }\end{array}$ & $1.90 \mathrm{E}-02$ & 9 \\
\hline GO Biological Process & GO:0042742 & $\begin{array}{l}\text { defense response to } \\
\text { bacterium }\end{array}$ & $2.18 \mathrm{E}-02$ & 8 \\
\hline GO Biological Process & GO:0070309 & lens fiber cell morphogenesis & $2.35 \mathrm{E}-02$ & 3 \\
\hline GO Biological Process & GO:0042330 & taxis & $2.62 \mathrm{E}-02$ & 13 \\
\hline GO Biological Process & GO:0016477 & cell migration & $2.49 \mathrm{E}-02$ & 27 \\
\hline GO Biological Process & GO:0042221 & response to chemical & $3.17 \mathrm{E}-02$ & 31 \\
\hline GO Biological Process & GO:0048870 & cell motility & 4.05E-02 & 27 \\
\hline GO Biological Process & GO:0034097 & response to cytokine & 4.32E-02 & 6 \\
\hline GO Biological Process & GO:0040011 & locomotion & $4.35 \mathrm{E}-02$ & 28 \\
\hline GO Biological Process & GO:0051336 & $\begin{array}{l}\text { regulation of hydrolase } \\
\text { activity }\end{array}$ & $4.28 \mathrm{E}-02$ & 17 \\
\hline GO Cellular Component & GO:0005773 & vacuole & $3.01 \mathrm{E}-02$ & 13 \\
\hline GO Cellular Component & GO:0005768 & endosome & $4.41 \mathrm{E}-02$ & 14 \\
\hline GO Cellular Component & GO:0009897 & $\begin{array}{l}\text { external side of plasma } \\
\text { membrane }\end{array}$ & 4.49E-02 & 9 \\
\hline
\end{tabular}




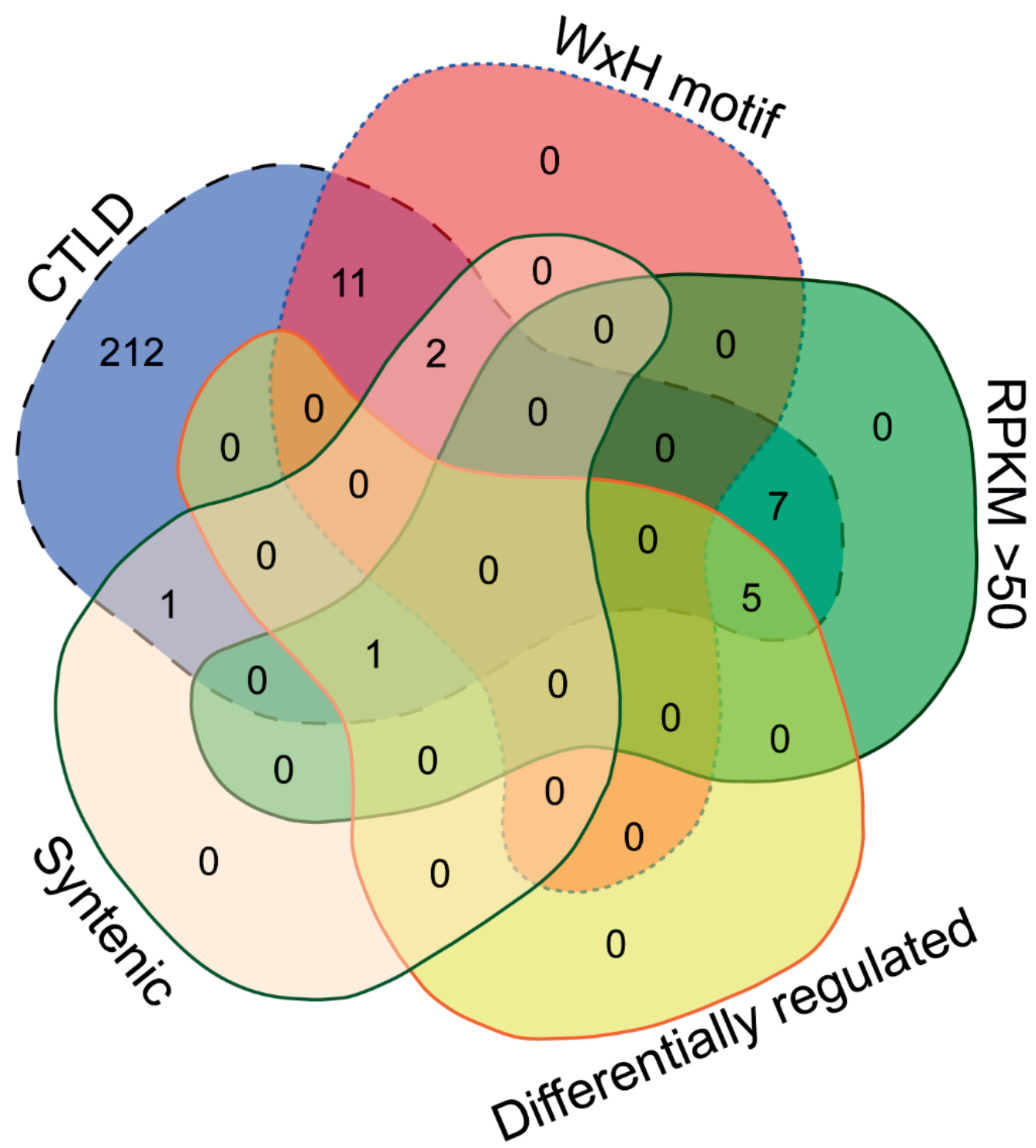

Supplementary figure 1 . Venn diagram visualizing all C-type lectin domain (CTLD) encoding genes and the separate filtering criteria for potential $\beta$-glucan receptor candidates. All CTLD-encoding genes are identified with a protein family (Pfam) search of the protein sequence (blue shape). Four independent criteria were used to highlight candidate $\beta$-glucan receptors genes: 1 ) conservation of the glucan binding $\mathrm{WxH}$-motif in the CTLD (red shape); 2) expression higher than 50 RPKM in carp macrophages (green shape);3) differential regulation by stimulation of carp macrophages with $\beta$-glucans (yellow shape); 4) CTLD-encoding genes located in a region with conserved synteny to the mammalian CLR group V cluster are highlighted (orange shape). 



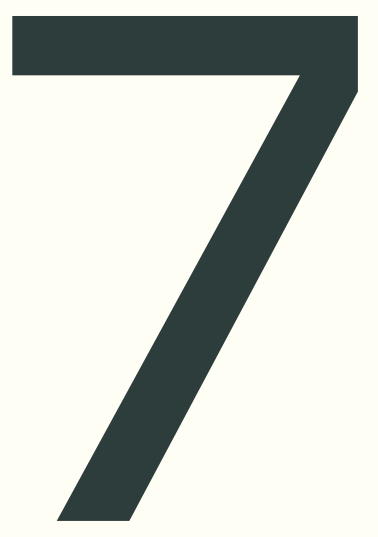

General discussion

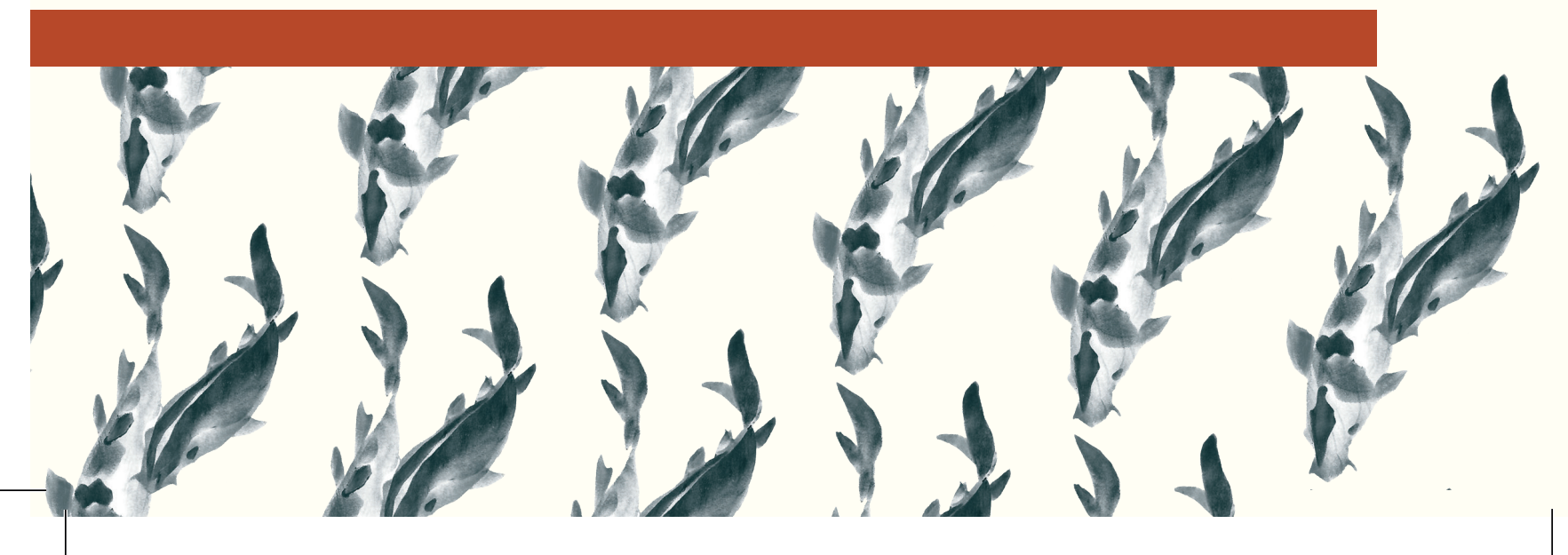


The tremendous growth of the aquaculture sector seen over the past decennia is associated with an intensification of the production process. This has led to an increase incidence of infectious diseases. To prevent these undesired effects, it has become relatively common to supplement fish feed with immuno-stimulants such as yeast-derived $\beta$-glucans. Several studies have reported generally positive effects, including increased resilience to stress and bacterial infections. Yet, to explain current effects of $\beta$-glucan supplementation, there is a need for a better understanding of the underlying mechanisms influencing the immune system. In the general introduction, we proposed to investigate three different mechanisms that could play a part in the observed effects of $\beta$-glucan supplementation in fish:

1. Long-lived effects on myeloid cells, typical of trained immunity;

2. Degradation and fermentation of $\beta$-glucans and associated shifts in composition of the intestinal microbiota and their metabolites;

3. Regulation of downstream signalling upon $\beta$-glucan recognition in carp macrophages

Looking back, we started with reviewing the literature for potentially overlooked, long-lived effects of $\beta$-glucan supplementation that could possibly be ascribed to trained immunity [Chapter 2, (20)]. Next, we established an in vitro model of trained immunity that led us to conclude that not only the NOD-like receptor ligand PGN, but also the soluble $\beta$-glucan laminarin, can induce trained immunity in common carp macrophages (Chapter $\mathbf{3}$ ). The second mechanism addressed was the ability of common carp intestinal microbiota to ferment $\beta$-glucans, resulting in the production of potentially immunomodulating short chain fatty acids (Chapter 4). The third mechanism dealt with the direct recognition of $\beta$-glucans and subsequent signalling, which was studied using next generation sequencing (NGS) [Chapter 5, (389)]. The subsequent study confirmed the regulation of the C-type lectin receptor (CLR) signalling pathway and led to the identification of several CLRs as potential candidates for recognition of $\beta$-glucans [Chapter 6, (41)].

Looking forward, here I discuss the implications of these findings and will position them in a theoretical framework against relevant literature. I will not only discuss the three proposed mechanisms, but also discuss the possible relations between these three as well as new mechanisms. Finally, I will elaborate on the relevance of my thesis by addressing the following question: "Will the description of mechanisms underlying immuno-modulatory properties of $\beta$-glucan have an impact on current practise in aquaculture?"

\section{Trained immunity: a critical reflection and outlook}

Although the basic mechanisms leading to the induction of trained immunity in human monocytes and carp macrophages may be comparable, there are also differences in approaches and read-outs. For example, mammalian monocytes 
trained with particulate Candida derived $\beta$-glucans produce reduced amounts of ROS upon non-specific stimulation (168), whereas carp macrophages trained with soluble Laminaria $\beta$-glucans produced increased amounts of ROS. Possibly, this outcome could be ascribed to differences between these two training stimuli with regard to solubility and/or origin, certainly when realizing that different preparations of laminarin can already result in different immune responses in mammalian macrophage cell lines (406). Alternatively, this outcome could be ascribed to differences between monocytes and macrophages. The majority of in vitro studies on trained immunity in mammals have been performed with isolated monocytes, while we studied a population of mature macrophages not devoid of progenitors $(164,407,408)$. Noteworthy, the common carp myeloid cell culture is derived from head-kidney, the haematopoietic organ in fish equivalent to mammalian bone marrow (409). Of interest, a recent study in mice showed that $\beta$-glucan-induced trained immunity can alter haematopoiesis, illustrated by an increased frequency of myeloid biased multipotent progenitor cells in the bone marrow and a more pronounced response to LPS of myeloid cells pre-exposed to $\beta$-glucans (410). The effect of $\beta$-glucans on myeloid cell progenitors, is an area of interest that deserves more attention, especially when it comes to long-lived effects associated with trained immunity.

From a fundamental perspective, the induction of trained immunity in carp macrophages is of high interest because it provides insight in the evolutionary conservation of innate immune responses to $\beta$-glucans. From an applied perspective, it can be equally interesting to enhance innate immune responses given the constantly high pathogen pressure in intensive aquaculture. Trained immunity in fact, is defined as a form of innate immune memory that results in enhanced inflammatory and antimicrobial properties of innate immune cells, providing an increased nonspecific response to subsequent infections and improved survival of the host (411). The non-specific nature of trained immunity especially, strongly supports observations on increased resistance to bacteria $(103,105)$, viruses $(412,413)$ and parasites $(94,97)$ upon dietary $\beta$-glucan supplementation. Evidence for antigen sampling in teleost intestine $(93,186)$ provides a cellular basis to these observations in fish, but in humans oral supplementation of $\beta$-glucans did not induce trained immunity (141). Yet, in our review (20) we could identify seven field studies where indications for long-lived effects of $\beta$-glucans were observed, three of which used oral supplementation of $\beta$-glucans $(103,155,156)$. These studies would suggest that induction of trained immunity via oral administration of $\beta$-glucans might be possible, at least in fish.

To date, several studies have shown that the ability to induce trained immunity is not unique to $\beta$-glucans, nor to Bacillus Calmette-Guérin (BCG), another common stimulus associated to trained immunity in mammals [reviewed by (411)]. Preexposure of human monocytes to endogenous oxidized low-density lipoprotein (oxLDL) (414), fungal chitin (415), raw milk or specific milk proteins (416), parasite infected red blood cells (417), all appear able to induce trained immunity. In addition, several cellular metabolites have been shown to induce trained 
immunity-like profiles in myeloid cells, such as fumarate, a substrate of the tricarboxylic acid cycle and mevalonate, an intermediate in the cholesterol synthesis pathway (59-61), highlighting the links between metabolism and innate immunity and providing new avenues for the (fish) feed industry to develop immuno-modulatory feeds.

Future studies addressing the potential for reprogramming of the innate immune system, should also address physiological costs of trained immunity. In mammals, maladaptive (trained) immunity can result in tissue damage or chronic inflammation [reviewed by: $(418,419)]$. For example, while exposure to oxidized LDL (oxLDL) can induce trained immunity-like characteristics (414), the associated long-term epigenetic reprogramming and the role of oxLDL in the development of artherosclerosis, might explain the persistent inflammatory profile of macrophages in atherosclerotic plaques. Recently, a study in mice connected Western diet and systemic inflammation with trained immunity. The authors observed long-lived transcriptional and epigenetic reprogramming, development of potentially harmful monocytes and an immunological over-reaction which might result in a maladaptive trained immunity response or in auto-immune disorders (420). It may be evident that there can be down sides to trained immunity related to a risk of persistent inflammation and associated tissue damage, but also increased metabolic costs of trained immunity should be considered. Sustained inflammatory responses can change the allocation of nutritional and energy resources with negative effects on growth efficiency $(421,422)$. It will thus be important to address the dynamics of trained immunity induced by dietary supplementation with $\beta$-glucans and study the physiological costs of feed supplementation strategies in aquaculture.

From a fundamental perspective, it is of interest to take the in vitro observations on trained immunity further and study innate immune responses in vivo, for example in $\mathrm{rag}^{-/-}$zebrafish. These mutant lines do not have functional B- and Tcells and thus no functional adaptive immune response (160). A tentative design could be as follows. First, trained immunity could be induced by a single intraperitoneal (i.p.) injection of $\beta$-glucans. Subsequently, fish would be exposed to different pathogenic challenges at different time intervals, for example at 7,14 , 21 , and 28 days post injection. The different challenges are of interest because of differences in innate memory in $\mathrm{rag}^{-/-}$zebrafish observed upon challenge with intracellular (152) or extracellular bacteria (160). The different time intervals are of interest because of earlier observations in zebrafish challenged with spring viremia carp virus showing effects at 7 but not 35 days post-injection with $\beta$-glucan (158). Preferably, the aforementioned experiments could be performed in crosses between $\mathrm{rag}^{-/}$zebrafish and transgenic zebrafish with fluorescent neutrophils (423) or macrophages (424) and for example combined with flow cytometry-based ROS production in "trained fish". This combination of approaches could confirm conservation of trained immunity in fish in vivo, validate the use of a single $\beta$ glucan i.p. injection to induce trained immunity and provide insight in the kinetics of trained immunity in fish. 
From an applied perspective, field studies on trained immunity should probably combine several approaches to validate assumptions made in individual studies; the whole will be greater than the sum of the parts, and a tentative design could be as follows. Using i.p. injection with $\beta$-glucan as a positive control, fish could be fed diets supplemented with different concentrations of $\beta$-glucans administered at different frequency and duration of exposure. As a crucial part, all groups should be switched to a control diet for a certain period (resting period, as in Chapter 3) to allow the organism to clear the presence of $\beta$-glucans, followed by challenge with different pathogens at different time intervals, as suggested above. This would provide insight in the duration of trained immunity "memory" in vivo. In larger-sized fish, innate immune cells could be sampled for ex vivo measurement of reactivity to stimulation with different PAMPs, as in previous studies using headkidney derived carp macrophages (164). Yet, field studies can never fully exclude a role of non-innate immune cells in the induction of trained immunity, no matter how carefully designed the experiment is. Given that a recent study showed that $\mathrm{T}$ cell-derived IFNy could be crucial for induction of trained immunity, at least in alveolar macrophages (425), "contamination" with other cell types (e.g. lymphocytes) during field studies on trained immunity may be considered irrelevant to those interested in practical applications and rightfully be considered of academic interest.

\section{Intestinal function: from microbiology to immunity}

Prebiotics are defined as ingredients selectively fermented by the intestinal microbiota affecting the composition and activity of the microbiota itself (26). They are considered ingredients that could be important for "feeding the microbiota". Probiotics are defined as live microorganisms that can provide health benefits when consumed, generally by improving or restoring intestinal microbiota (426). While there is appreciation of the beneficial effects of prebiotics and probiotics by the aquaculture sector [reviewed by: (427-430)], a knowledge gap remains on the underlying mechanisms of such health benefits. In mice, a connection was made between a high-fibre diet and reduced allergy-induced inflammation in the lungs acting via short-chain fatty acids (SCFAs) produced by the microbiota (231).

SCFAs are fatty acids with less than six carbon atoms and produced as end products of fermentation of dietary fibres by the intestinal microbiota. SCFAs can have modulatory effects on inflammation via two major but different routes: 1) through receptor independent inhibition of histone de-acetylases (HDACs) of the host; 2) through activation of host SCFA receptors such as G-protein coupled receptor (GPR) 41, GPR43 and GPR109A [also known as free fatty acid receptor (FFAR) 3, FFAR2 and hydroxycarboxylic acid receptor 2 (HCAR2)]. Neither of the two routes have been described in detail in fish nor has modulation of the immune system by SCFAs produced by intestinal microbiota been widely studied in fish. 
Histone de-acetylases (HDACs) are an evolutionary-conserved family of proteins present from bacteria to humans. HDACs mediate hypo-acetylation which is generally associated with a closed chromatin structure, thereby blocking or repressing gene transcription due to the inaccessibility of promotor regions for transcription factors [reviewed by: $(72,431,432)]$. The presence of SCFAs can inhibit HDACs and thus result in hyper-acetylation, which is associated with an open chromatin structure, facilitating the binding of transcription factors to promotor regions. The effects of these epigenetic changes can be diverse, dependent on the region of acetylation. Within an immunological context, butyrate and propionate are currently thought to be the two most important SCFAs. Butyrate and propionate have both been shown to inhibit LPS-induced TNF-a production via inhibition of NF-KB and increased IL10 production $(73,433)$. Moreover, butyrate was shown to reduce phagocytosis and ROS production by neutrophils (434). Given that epigenetic reprogramming has been claimed an important factor driving trained immunity [as reviewed by: (181)] and given that ROS and TNFa are inflammatory factors frequently associated with trained immunity (this thesis), it is of interest to study the potential influence of SCFAs on trained immunity in fish.

SCFA receptors have not yet been described in fish. In zebrafish, four predicted genes on chromosome 16 and three predicted genes on chromosome 19 are currently associated with FFAR2 and 3, however no validation nor characterization of these genes has been performed. In the latest common carp assembly, $n=4$ genes are annotated as mammalian FFAR 2 and $n=8$ genes are annotated as mammalian FFAR3. In human and mouse monocytes, both FFAR2 and FFAR3 are constitutively expressed but down-regulated upon differentiation into macrophages (435). Our NGS data set of carp macrophages showed constitutive expression of two putative ffar genes with reasonable values of 27-47 reads per kilobase million (RPKM), corresponding to $0.5-1 \%$ of the constitutive $\beta$-actin expression. One of these two genes was even up regulated in macrophages stimulated with MacroGard ${ }^{\circledR}$. This suggests that innate immune cells of carp such as macrophages could possibly express SCFA receptors and might be responsive to modulation by SCFAs such as butyrate and propionate. In a prelimary experiment we investigated the modulatory effect of different SCFAs, in vitro, on LPS-stimulated head kidney leukocytes (HKL) of carp (Figure 1). Butyrate in particular had a clear immunomodulatory effect.

In light of the presence of high number of macrophages in the carp intestine (187), it appears reasonable to hypothesize that SCFAs produced in response to shifts in the composition of intestinal microbiota, can be recognized by receptors present on these macrophages, thereby modulating local immune responses. Of course, since the current annotation of the carp genome is still largely based on prediction and previous de novo transcriptome data $(253,255)$, the next step should be validation of the putative ffar genes in carp. Only following their validation as receptors for SCFAs, subsequent studies can address organ expression and receptor dependent effects of different SCFAs. Clearly, receptor independent 
effects of SCFAs, for example on HDACs, should not be neglected and both routes of action should be addressed in future studies.

A $\square$ RPMI $\square$ LPS

B $\square$ RPMI $\square$ LPS
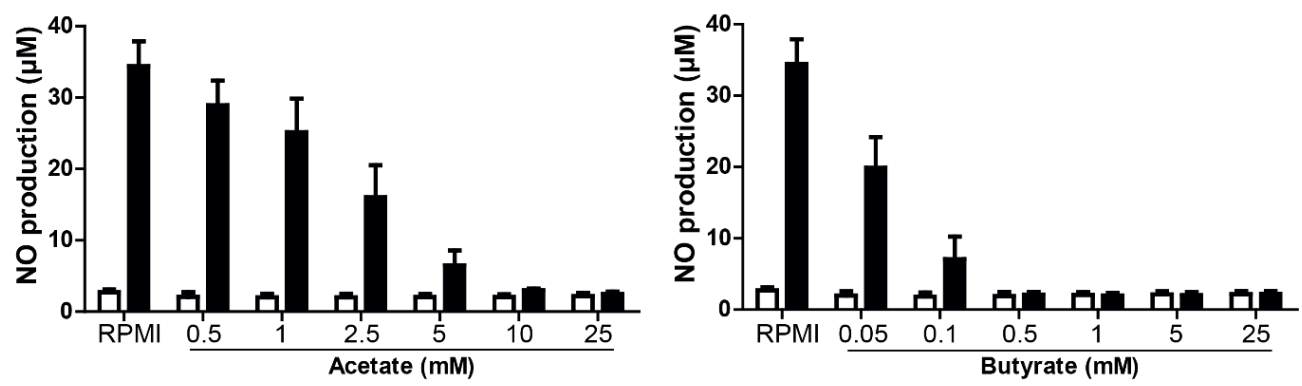

C

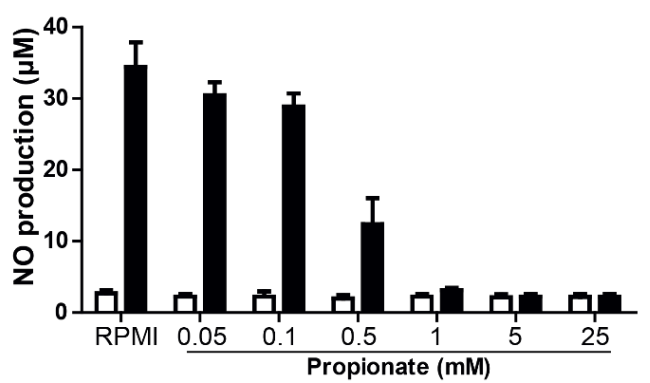

Figure 1. SCFA reduce LPS-induced production of nitric oxide (NO) in head kidney leukocytes (HKL). Cells were stimulated with LPS $(30 \mu \mathrm{g} / \mathrm{mL})$ for $96 \mathrm{hrs}$ in the presence or absence of the SCFAs, acetate, butyrate and propionate. A. NO production of LPSstimulated HKL in the presence of acetate. B. NO production of LPS-stimulated HKL in the presence of butyrate. C. NO production of LPS-stimulated HKL in the presence of propionate. Bars indicate mean $+S D$ of $n=3$ independent experiments.

\section{Regulation of the CLR pathway: zooming in}

A genome-wide study could not identify true $C$-type lectin super family $V$ members in the genome of Fugu (Takifugu rubripes) (42), and neither did we observe clustering of carp C-type lectin domain (CTLD) sequences with CTLD sequences from mammalian family $\mathrm{V}$ members (41). The receptor for $\beta$-glucans therefore may not be conserved between mammals and fish, but we did observe regulation of several elements of the $\mathrm{C}$-type lectin receptor signalling pathway upon $\beta$-glucan stimulation in carp macrophages (Figure 2). Interestingly, we observed regulation of all three components of the Card9-Bcl10-Malt1 signalling complex, which strongly hints towards involvement of this complex in the response to $\beta$-glucan. This signalling complex is activated by $\mathrm{PKC} \delta$ that is recruited by activated syk, downstream of Dectin-1. In contrast, we observed a down-regulation of syk following stimulation of macrophages with $\beta$-glucans $(40,44)$, which might suggest signalling via a syk- independent mechanism. In this study two different $\beta$-glucan preparations were used: curdlan, a linear $\beta-(1,3)$-glucan considered a Dectin-1specific ligand in mammals, and MacroGard ${ }^{\circledR}$, a branched $\beta-(1,3 / 1,6)$-glucose 


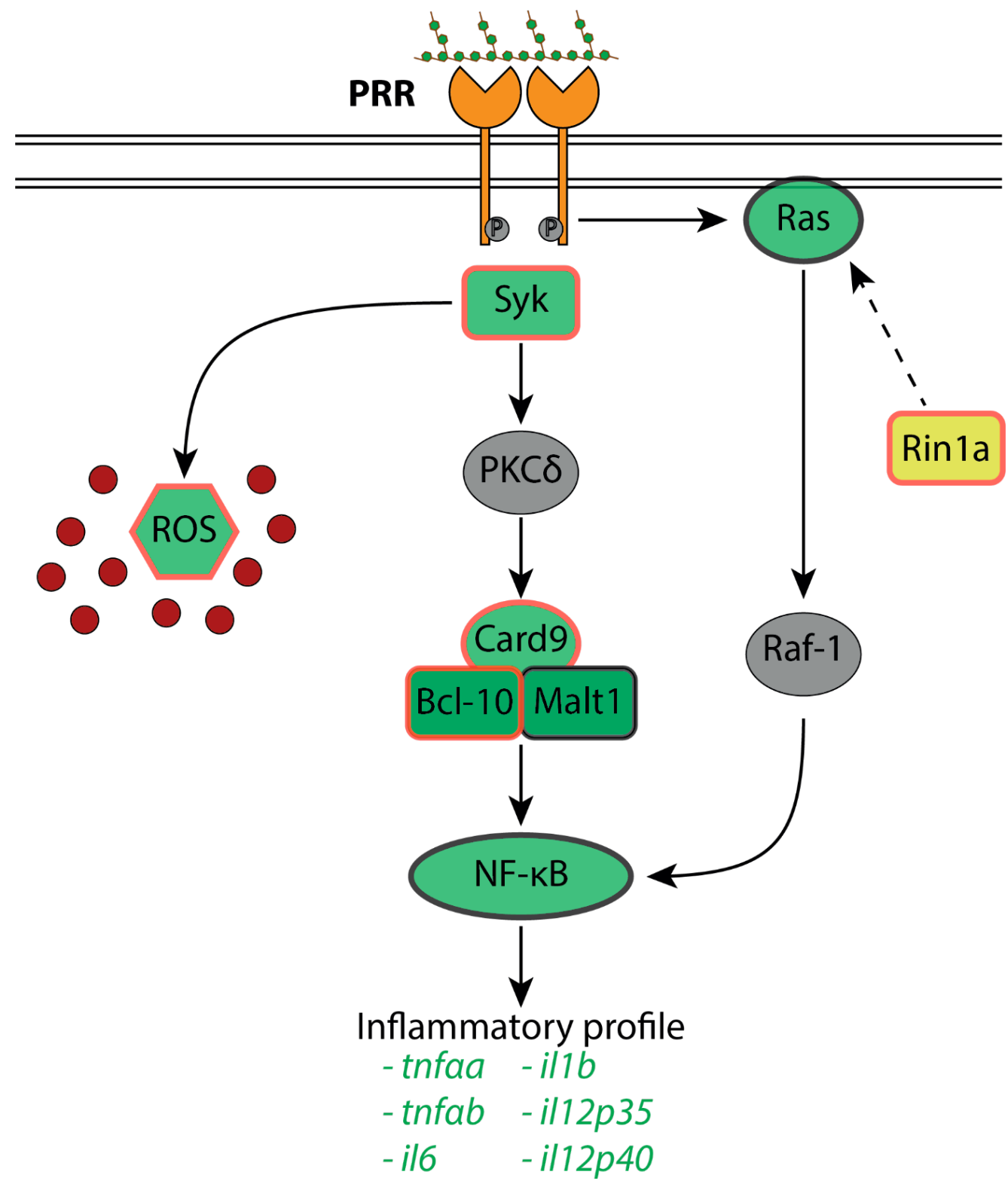

Figure 2. $\beta$-glucan regulated carp genes superimposed on the mammalian Dectin1 signalling pathway. Orange PRRs represent proposed candidates based on the investigation of CLR family members in the carp genome. Signalling molecules depicted in green were identified as genes regulated in macrophages stimulated with $\beta$-glucans (Chapter 6). Signalling molecules depicted in grey were not regulated. Molecules marked by a red border were also observed regulated in separate experiments, either functional (ROS production) or by qPCR (syk, card9 and $b c /-10)$. Gene regulation of the list under inflammatory profile was validated by qPCR. The yellow Rin 1 a was specifically regulated at transcription level. Rin1a can inhibit Ras-regulated Raf-1 activation. 
widely-applied as feed additive in aquaculture. We later included a third $\beta$-glucan preparation zymosan, a commonly used yeast preparation and compared the differentially expressed genes (DEGs) regulated by all three different $\beta$-glucan preparations. By comparing the overlap in regulation between the three datasets we aimed to identify a common $\beta$-glucan-regulated gene set (Figure $3 \mathrm{~A}$ ). Although the absolute number of genes regulated by zymosan $(n=224)$ was lower than for MacroGard $^{\circledR}(n=789)$ or curdlan $(n=528)$, there was a major overlap in DEGs, indicative of a common $\beta$-glucan-regulated gene sets.

A

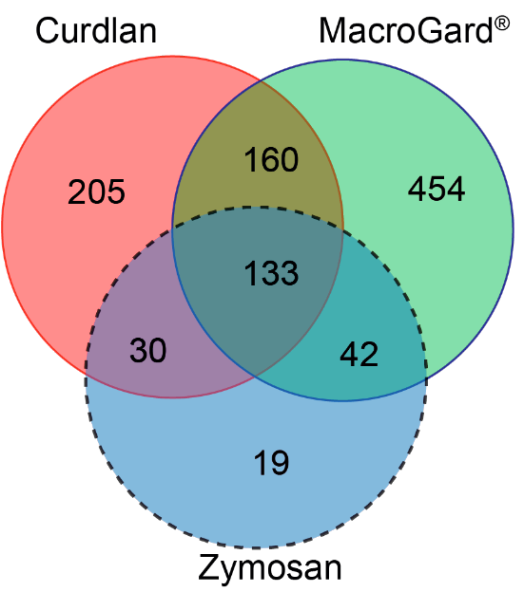

B

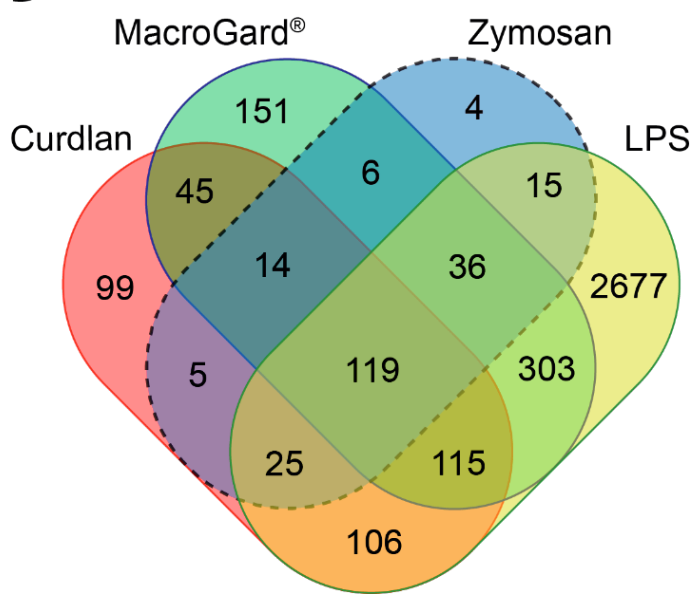

Figure 3. Venn diagram comparing differentially expressed genes (DEGs) regulated upon stimulation of common carp macrophages with different PAMPs. A. Venn diagram of the DEGs regulated by stimulation of macrophages with three different $\beta$-glucan preparations. Macrophages were stimulated for 6 hours with $25 \mu \mathrm{g} / \mathrm{mL}$ of either curdlan, MacroGard ${ }^{\circledR}$ or Zymosan and collected for RNAseq analysis. B. Venn diagram comparing the DEGs regulated by the three different $\beta$-glucan preparations shown in $A$. with DEGs regulated by stimulation of macrophages with LPS $(30 \mu \mathrm{g} / \mathrm{mL})$ for $6 \mathrm{~h}$.

Comparison of the DEGs regulated by all three $\beta$-glucan preparations with an unpublished DEG dataset of macrophages stimulated with LPS (Figure 3B), identified 14 unique $\beta$-glucan-regulated genes not present in the LPS dataset (Table 2). Of special interest is rin1a; orthologous to mammalian Rin1 (Rab and Ras interactor protein). In mammals, Rin1 can directly interact with Ras and a membrane trafficking GTPase Rab5, regulating receptor-dependent endocytosis (436). Furthermore, Rin1 and Raf-1 actually compete for binding of Ras, and possibly the signal transduction of Ras is determined by the competition between Rin1 and Raf-1 (437). Although the conservation of the interactions between Rin1a, Raf-1 and Ras has not been confirmed in fish, the up-regulation of rin1a again hints at a role for the syk-independent pathway (Figure 2). 
Table 1. Tentative role of genes uniquely regulated in carp macrophages stimulated with $\boldsymbol{\beta}$-glucans. List of DEGs all regulated in carp macrophages by three different $\beta$-glucan preparations but not in macrophages stimulated with LPS for $6 \mathrm{~h}$, identifying 14 unique $\beta$-glucan-regulated genes not present in the LPS dataset. Order of genes based on cypCar code (not shown).

\begin{tabular}{|c|c|c|}
\hline Gene code & Description & Putative relevance \\
\hline$s h 2 d 3 c b$ & $\begin{array}{l}\text { SH2 domain containing } 3 \mathrm{Cb} \\
\text { (Chat) }\end{array}$ & $\begin{array}{l}\text { Involved in monocyte adherence and } \\
\text { regulation of lymphocyte adherence (438, } \\
439) \text {. }\end{array}$ \\
\hline ripk2 & $\begin{array}{l}\text { Receptor-interacting } \\
\text { serine/threonine-protein } \\
\text { kinase } 2\end{array}$ & $\begin{array}{l}\text { Regulator of inflammatory responses and } \\
\text { involved in multiple different pathways in } \\
\text { innate and adaptive immune response, } \\
\text { among others TLR, NLR and IL1R } \\
\text { signalling (440). }\end{array}$ \\
\hline fth/31 & $\begin{array}{l}\text { ferritin, heavy polypeptide- } \\
\text { like } 31\end{array}$ & $\begin{array}{l}\text { Macrophages are a major source of serum } \\
\text { ferritin in mammals and increased serum } \\
\text { ferritin levels can be associated to } \\
\text { inflammation (441). }\end{array}$ \\
\hline il10ra & $\begin{array}{l}\text { interleukin } 10 \text { receptor, } \\
\text { alpha }\end{array}$ & $\begin{array}{l}\text { Involved in the regulation of inflammation } \\
\text { ( } 442) \text {. Upregulated upon murine lungs } \\
\text { exposed to curdlan ( } 443 \text { ). }\end{array}$ \\
\hline ctsla & Cathepsin La & $\begin{array}{l}\text { Differentially expressed in antigen } \\
\text { presenting cells and involved in regulating } \\
\text { MHC class II presentation }(444,445) \text {. }\end{array}$ \\
\hline$B c / 2 / p 14$ & $\begin{array}{l}\text { Apoptosis facilitator } \mathrm{Bcl}-2- \\
\text { like protein } 14\end{array}$ & Regulator of apoptosis facilitation (446). \\
\hline$c d k n 1 c b$ & $\begin{array}{l}\text { Cyclin-dependent kinase } \\
\text { inhibitor } 1 \mathrm{Cb}\end{array}$ & $\begin{array}{l}\text { Negative regulator of proliferation and } \\
\text { highly downregulated in M2 macrophages } \\
(447) \text {. }\end{array}$ \\
\hline$f 3 b$ & Coagulation factor IIIb & $\begin{array}{l}\text { F3 enables the initiation of blood } \\
\text { coagulation cascades, furthermore, F3 can } \\
\text { induce M2-like profile in macrophages } \\
(448) \text {. }\end{array}$ \\
\hline zgc92242 & Uncharacterized protein & $\begin{array}{l}\text { This gene is identified based on cDNAs } \\
\text { isolated as part of the Zebrafish Gene } \\
\text { Collection (ZGC) but not further } \\
\text { characterized. }\end{array}$ \\
\hline anxa2a & Annexin $\mathrm{A} 2 \mathrm{a}$ & $\begin{array}{l}\text { Annexin A2 tetramers can act as } \\
\text { recognition protein for bacterial and viral } \\
\text { pathogens and induce TNFa, IL1 } \beta \text { and IL6 } \\
(449) \text {. }\end{array}$ \\
\hline rsad2 & $\begin{array}{l}\text { radical S-adenosyl } \\
\text { methionine domain } \\
\text { containing } 2 \text { (viperin) }\end{array}$ & $\begin{array}{l}\text { Important interferon stimulated gene with } \\
\text { antiviral properties [reviewed by: }(450) \text { ] } \\
\text { and potentially involved in macrophage } \\
\text { polarization ( } 451) \text {. }\end{array}$ \\
\hline siglec12 & $\begin{array}{l}\text { sialic acid-binding Ig-like } \\
\text { lectin } 12\end{array}$ & $\begin{array}{l}\text { CD33 related siglec, but its natural ligand } \\
\text { remains to be determined ( } 452) \text {. }\end{array}$ \\
\hline tpcn2 & $\begin{array}{l}\text { Two pore segment channel } \\
2\end{array}$ & $\begin{array}{l}\text { Sodium ion channel on intracellular } \\
\text { endosomes and lysosomes (453). }\end{array}$ \\
\hline
\end{tabular}




\section{Binding proteins, an underappreciated group of receptors}

Several pattern recognition proteins (PRPs) have been described in invertebrate that are capable of recognizing pathogen epitopes and are able to bind $\beta$-glucans, such as $\beta 1,3$-Glucan recognition protein ( $\beta$ GRP)/Gram-negative bacteria-binding protein 3 (GNBP3) and Lipopolysaccharide- and $\beta$-1,3-Glucan-binding Protein (LGBP) [reviewed by: $(21,89,454)]$. However, a motif search in the annotated carp genome [BioProject: PRJNA73579, (255)], using CLC Main workbench ${ }^{5}$ and a conserved $\beta G R M^{6}$ motif based on a comparison of the putative $\beta$-glucan recognition motif of six different invertebrate LGBPs, provided no significant hits. Yet, considering the evolutionary position of teleost fish as early vertebrates and the apparent absence in fish genomes of genes orthologous to mammalian Dectin-1, it is plausible that fish can (also) express receptors similar to those described in invertebrates.

The family of PGRPs is present in genomes from insects to mammals with diverse functions in antimicrobial defence (455). In the genome of Drosophila melanogaster, where PGRPs are extensively studied, 13 different pglyrp encoding genes are present. These can be spliced into 19 different proteins, classified into secreted PGRP-short (S) or PGRP-long (L) that are either secreted, transmembrane, or intracellular (456). A study in beetle, Holotrichia diomphalia, reported binding of $\beta-(1,3)$-glucans by two secreted PGRPs with high sequence similarity to human PGLYRP-1, and activation of subsequent innate immune responses (457). In mammals, PGRPs were first classified as short, long and intermediate in analogy to insect PGRPs, but have been renamed into PGLYRP-1, $-2,-3$ and -4 . In fish, currently, three genes have been identified, pglyrp-2, -5 and -6 , with pglyrp-2 and -6 showing the closest phylogenetic relation to PGLYRP-2 and pglyrp-5 showing the closest relation to PGLYRP-1 (458). One study in grass carp studied the binding of Pglyrp proteins to $\beta$-glucans (459). In this study, four splice variants of grass carp Pglyrp- 6 were shown to interact with $\beta$-glucans and with $S$. cerevisiae yeast cells, potentially suggesting a role for Pglyrp proteins in recognition of or binding to $\beta$-glucans. More recently, a study reported the identification of common carp pglyrp-5 and -6 (460), but no functional characterization was performed of the identified genes. We could identify four different carp pglyrp genes; pglyrp-2 and -6, and two paralogs of pglyrp-5 in the most recent common carp genome assembly. Based on RNAseq analysis ${ }^{7}$, we found that none of these genes were constitutively expressed in carp macrophages. However, in two out of three RNAseq data sets from macrophages

\footnotetext{
5 https://www.qiagenbioinformatics.com

6 Conserved sequence used as search query: FHxWRxDWTxxxMxxYVDx

7 The RNAseq dataset published in Chapter 6 was used for this analysis.
} 
stimulated with MacroGard ${ }^{\circledR}$, both pglyrp-5 paralogs were up-regulated. This prompted us to perform further experimental work.

Stimulation of carp macrophages with zymosan, curdlan or MacroGard ${ }^{\circledR}$ for $6 \mathrm{~h}$ resulted in increased gene expression of both paralogs of pglyrp-5 (Figure 3A). Stimulating carp macrophages with zymosan pre-treated with pglyrp5-1 and -2 induced increased ROS production (Figure 3B). These preliminary experiments show that $\beta$-glucans not only regulate pglyrp 5 expression but also that pretreatment of $\beta$-glucans with pglyrp5 can alter the response of macrophages to $\beta$ glucans. Although further research would be required, these preliminary data hint at a possibly conserved role of pglyrp 5 proteins in the response to $\beta$-glucans in fish.

\section{Are $\boldsymbol{\beta}$-glucans unique?}

This thesis focussed on the use of $\beta$-glucans to induce immuno-modulation. A possible alternative to the use of $\beta$-glucans can be the use of marine sulphated polysaccharides (MSPs). MSPs are biologically active components of marine algae (seaweeds), which are among the fastest growing plant organisms in nature. These

A

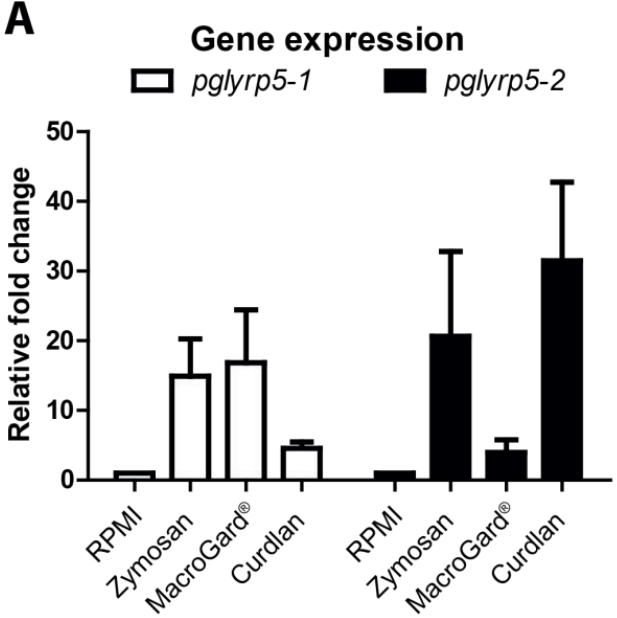

B

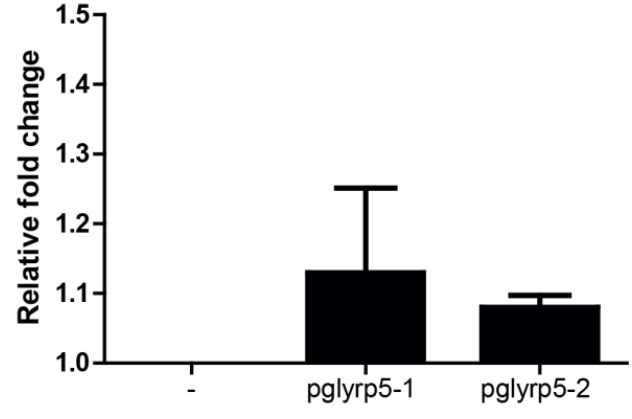

Zymosan $25 \mu \mathrm{g} / \mathrm{mL}$

Figure 4. Preliminary results on a relation between stimulation with $\beta$-glucans and Pglyrp5 activity in carp macrophages. Bars indicate mean + SEM of $n=3$ experiments performed independently. A. Expression of both paralogs of pglyrp5 was analysed with qPCR using paralog-specific primers. RNA was isolated from carp macrophages after $6 \mathrm{~h}$ stimulation with the indicated $\beta$-glucans $(25 \mu \mathrm{g} / \mathrm{mL})$. Fold change is calculated relative to macrophages stimulated with culture medium (RPMI). B. ROS production after stimulation of carp macrophages with Pgrp5-pre-treated zymosan (25 $\mu \mathrm{g} / \mathrm{mL}$ ). Zymosan was pre-treated for $1 \mathrm{~h}$ with transfection supernatant from VERO cells transfected with pcDNA3-empty (-), pcDNA3-pglyrp5-1 or pcDNA3-pglyrp5-2. Following incubation for $1 \mathrm{~h}$, zymosan was centrifuged, washed with fresh culture medium and used to stimulate macrophages. Fold change ROS production is expressed as area under the curve of ROS production for $2 \mathrm{~h}$ as measured with a luminol assay. Fold change is calculated relative to macrophages stimulated with zymosan pre-treated with supernatant from pcDNA3-empty-transfected VERO cells. 
macroalgae are widely investigated for their sugar and carbohydrate content (461) that can be fermented to produce biogas or ethanol-based bio-fuels, much alike the process of sugar cane fermentation used in Brazil (see introduction to this thesis). However, the presence of MSPs can severely inhibit the fermentation process, reducing the efficiency of ethanol or biogas production, providing a good reason to extract MSPs. Of interest here, a broad range of biological properties have been ascribed to MSPs, including anticoagulant, antiviral and broader immuno-modulatory activities.

MSPs are common to the cell wall of all marine algae, but their concentrations differ among the three major groups of marine algae, Rhodophyceae (red algae), Chlorophyceae (green algae), and Phaeophyceae (brown algae). Well-known MSPS from green algae are ulvans, highly sulphated water-soluble polysaccharides with a backbone composed of sulphate, rhamnose, xylose, iduronic and glucuronic acids. Ulvans have been thoroughly investigated for their immuno-modulatory effects in mammals and other warm-blooded vertebrates $(462,463)$, but their potential for the aquaculture sector has yet to be appreciated, illustrated by only few published studies in fish. In turbot (Scophthalmus maximus), ulvan stimulated the respiratory burst of leukocytes in vitro (464) and increased il1 $\beta$ expression (465), a pro-inflammatory activity that could be abolished by de-sulphation. In Nile tilapia (Oreochromis niloticus), dietary supplementation of ulvan could increase the white blood cell count and phagocytic activity of isolated leukocytes (466). It may be obvious that in fish the exact immuno-modulatory mechanisms induced by MSPs such as ulvan, are still largely unknown.

Similar to what was discussed in this thesis for $\beta$-glucans, effector and or recognition mechanisms important for immuno-modulation by ulvan might be (partially) conserved between warm-blooded vertebrates and fish. In chicken, a recent in vitro study showed that ulvan can activate monocytes after recognition by TLR2 and TLR4, leading to an increase in NO production and transcription of several cytokines, among which il1 $\beta$, ifna, ifny, il8 and inos (467). Furthermore, in vivo dietary supplementation of ulvans was immuno-modulatory. At present, conserved roles may be suspected but remain to be shown for most Tlrs in fish, and recognition of specific ligands has not been unambiguously proven for TIr2 nor for TIr4 [reviewed by: (134)]. Nevertheless, there is no reason to believe that MSPs should not have an immuno-modulatory effect on fish cells. Further studies into the exact immuno-modulatory effects of MSPs in fish should focus on innate immune cell types such as macrophages, their receptors and their functional responses.

\section{Are my data relevant to aquaculture practice?}

At the beginning of this discussion chapter, I promised to not only look forward by discussing the inter-relations and implications of my findings but also discuss the relevance of my thesis by focussing on the following question: "Will the description 
of mechanisms underlying immuno-modulatory properties of $\beta$-glucan have an impact on current practise in aquaculture?"

Throughout this chapter I already addressed this question in different sections and discussed: i) trained immunity as a form of innate immune memory that provides an increased nonspecific response to subsequent infections and improved survival of the host, the latter of obvious interest to aquaculture practise. I mentioned this could come at a metabolic cost that could have an impact on growth performance; the optimal energetic balance between metabolic cost for prevention of infections and fighting pathogens remaining unknown for now. Further I discussed that in field studies on trained immunity "contaminations" with cell types other than those of the innate immune system may be rightfully considered of academic interest only. ii) Prebiotics and their assumed beneficial effects on the immune system, possibly mediated by SCFAs produced by the intestinal microbiota, leading to nutritional concepts such as "feeding the microbiota", despite the current knowledge gap. iii) The use of other prebiotic polysaccharides such as MSPs and the striking parallels between ulvans from seaweed and $\beta$-glucans from yeast when it comes to bio-ethanol production and use of "waste" materials as feed additives for animals, including fish.

At present, products such as $\beta$-glucans and ulvans are mostly restricted to fish feed for species of high economic value in intensive aquaculture systems such as Atlantic salmon, mostly for economic reasons. Yet, for these species prevention of disease by vaccination is also common, and an alternative is not always available and/or economically viable for fish species kept in less intensive (pond) systems, such as tilapia. This would argue in favour of a new focus and associated marketing of "healthy feeds" at lower costs for the farmers interested in less intensive aquaculture systems. This might require use of immune-modulatory products of reduced purity, at lower production costs, the exact working mechanisms of which should be verified against the present "pure" products.

Over the last few years, access to fish genomes and thus the possibilities for NGSbased approaches to analyse fish immune responses have increased considerably. With the emergence of affordable bench-top equipment such as based on nanopore technology, large-scale RNA sequencing might rapidly replace specific detection by PCR and might even come to the farm. Yet, gene duplications common to fish genomes continue to complicate the correct interpretation of these large data sets and will require continued involvement of both, bioinformaticians and biologists. This is where my review of the current understanding of NGS approaches to the immune system of fish (389) may be relevant to practise.

Last but not least, looking ahead, improved understanding of the effects of dietary supplementation with immuno-modulatory compounds such as $\beta$-glucans or other could help achieve concepts such as "personalized feeding" based on the concept of "personalised nutrition". The ultimate goal of personalised nutrition is to enable individual guidance by predictive knowledge of personal health to diets that prevent disease and maximize health [reviewed by: (468)]. Improving fish health 
by personalised diets would reduce the need to employ other measures to improve health, such as antibiotics or expensive vaccination strategies. Yet, this goal is not easily applied to individual fish in aquaculture systems but could be applicable to individual species in aquaculture. This would imply optimisation of fish feed to requirements of species with different metabolic, physiological and/or genetic status, and more. In this thesis, I have used multiple approaches to provide a scientific base to achieve such goal on the long term, inspired by new accurate and predictive measures of health, based on molecular signatures of metabolites, proteins, transcripts, genes, and microbiota. 


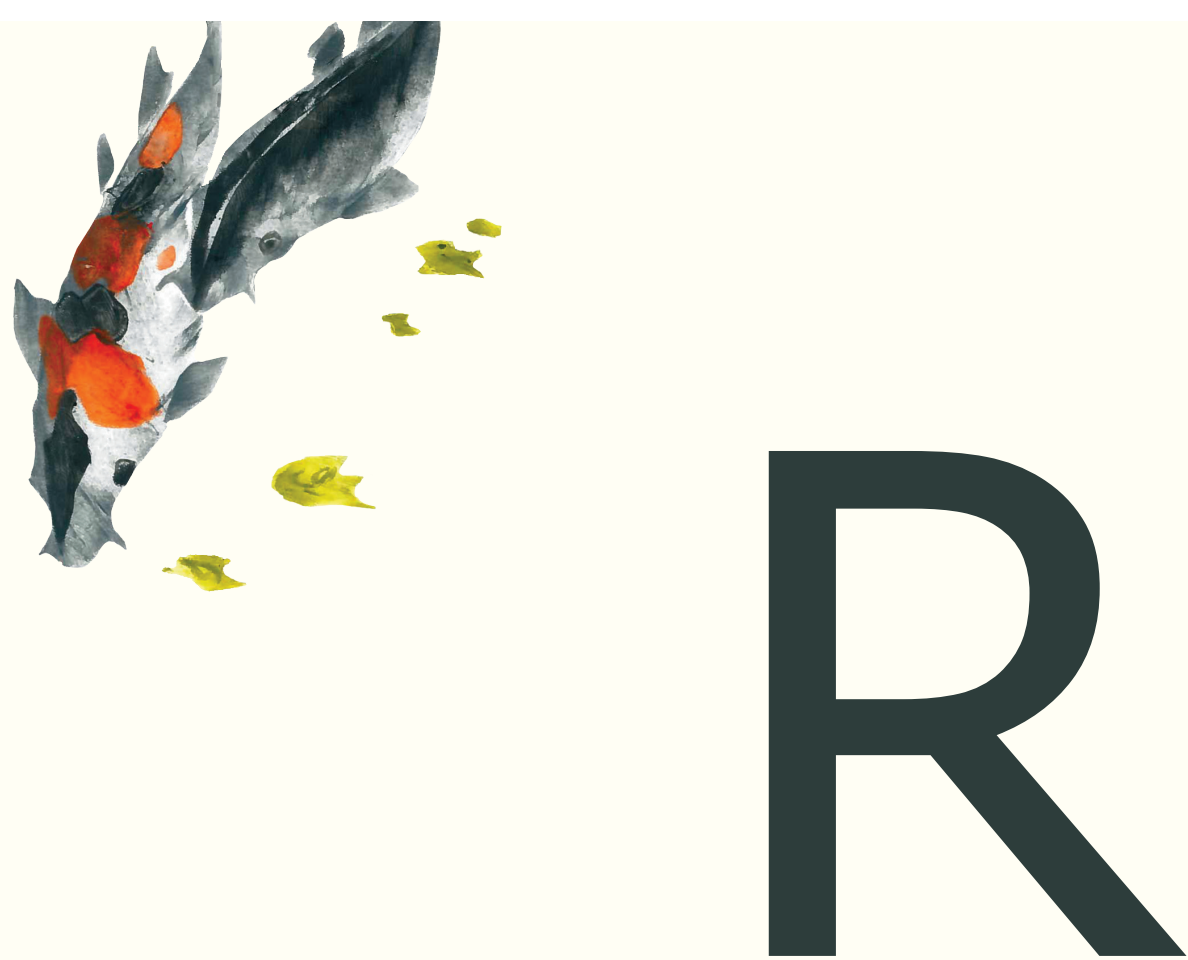

References

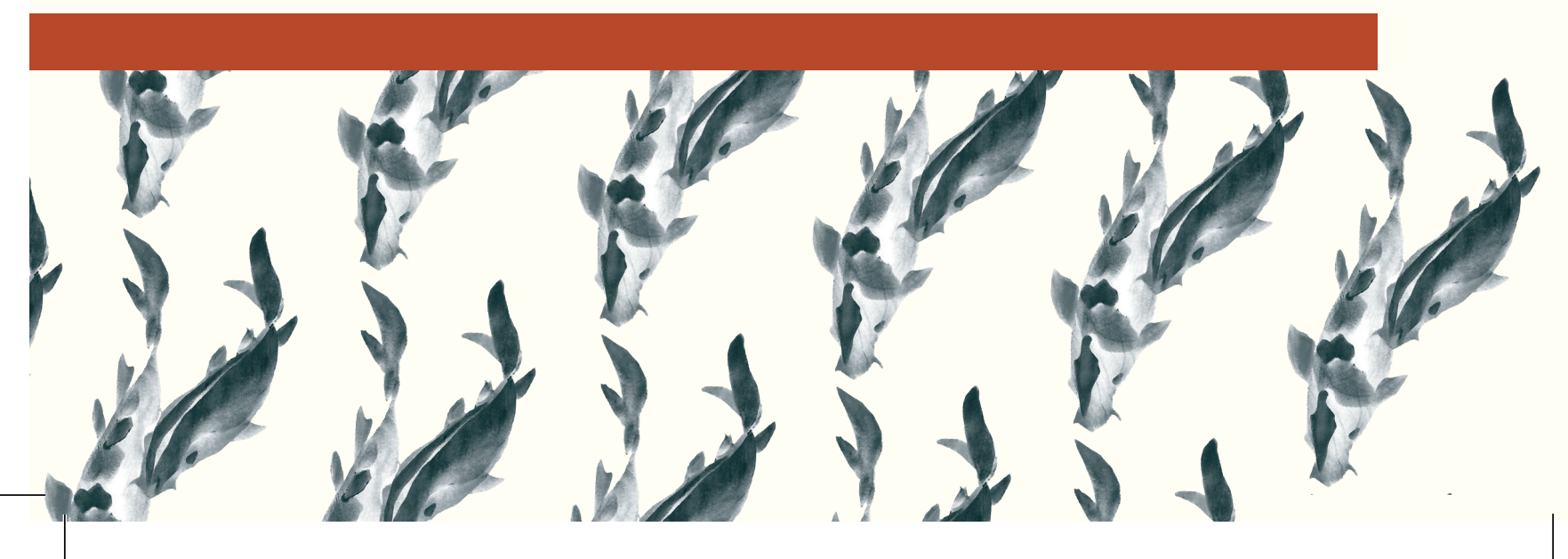


1. Goldemberg, J. 2008. The Brazilian biofuels industry. Biotechnology for biofuels 1: 6 .

2. Matsuoka, S., Ferro, J., and Arruda, P. 2009. The Brazilian experience of sugarcane ethanol industry. In Vitro Cell Dev-PI 45: 372-381.

3. Lopes, M. L., Paulillo, S. C. D., Godoy, A., Cherubin, R. A., Lorenzi, M. S., Giometti, F. H. C., Bernardino, C. D., Neto, H. B. A., and de Amorim, H. V. 2016. Ethanol production in Brazil: a bridge between science and industry. Braz J Microbiol 47: 64-76.

4. Basso, L. C., Basso, T. O., and Rocha, S. N. 2011. Ethanol production in Brazil: the industrial process and its impact on yeast fermentation. In Biofuel production-recent developments and prospects. InTech.

5. Kwiatkowski, S., and Kwiatkowski, S. E. 2012. Yeast (Saccharomyces cerevisiae) glucan polysaccharides-occurrence, separation and application in food, feed and health industries. In The complex world of polysaccharides. IntechOpen.

6. Rizzetto, L., Weil, T., and Cavalieri, D. 2015. Systems Level Dissection of Candida Recognition by Dectins: A Matter of Fungal Morphology and Site of Infection. Pathogens 4: 639-661.

7. Pietretti, D. 2013. Stimulation of the innate immune system of carp: role of Toll-like receptors. Wageningen University, Wageningen.

8. Fink, I. R. 2017. Innate immunity of carp : fishing for receptors. Wageningen University, Wageningen.

9. FAO. 2018. The State of World Fisheries and Aquaculture 2018: Meeting the sustainable development goals. FAO.

10. Barton, B. 2011. Stress in finfish: Past, present and future-a historical perspective. Cambridge, UK: Cambridge University Press.

11. Costas, B., Aragao, C., Mancera, J. M., Dinis, M. T., and Conceicao, L. E. C. 2008. High stocking density induces crowding stress and affects amino acid metabolism in Senegalese sole Solea senegalensis (Kaup 1858) juveniles. Aquac Res 39: 1-9.

12. Wedemeyer, G. 1997. Effects of rearing conditions on the health and physiological quality of fish in intensive culture. Fish stress and health in aquaculture: 35-71.

13. Kent, M. L. 2000. Marine netpen farming leads to infections with some unusual parasites. Int J Parasitol 30: 321-326.

14. Lafferty, K. D., Harvell, C. D., Conrad, J. M., Friedman, C. S., Kent, M. L., Kuris, A. M., Powell, E. N., Rondeau, D., and Saksida, S. M. 2015. Infectious Diseases Affect Marine Fisheries and Aquaculture Economics. Annu Rev Mar Sci 7: 471-496.

15. Tavares-Dias, M., and Martins, M. L. 2017. An overall estimation of losses caused by diseases in the Brazilian fish farms. Journal of Parasitic Diseases 41: 913-918.

16. Miranda, C. D., Godoy, F. A., and Lee, M. R. 2018. Current Status of the Use of Antibiotics and the Antimicrobial Resistance in the Chilean Salmon Farms. Front Microbiol 9.

17. Done, H. Y., Venkatesan, A. K., and Halden, R. U. 2016. Does the Recent Growth of Aquaculture Create Antibiotic Resistance Threats Different from those Associated with Land Animal Production in Agriculture? (vol 18, pg 513, 2016). Aaps J 18: 1583-1583.

18. Romero, J., Feijoó, C. G., and Navarrete, P. 2012. Antibiotics in aquaculture-use, abuse and alternatives. In Health and environment in aquaculture. InTech.

19. Dalmo, R. A., and Bogwald, J. 2008. Beta-glucans as conductors of immune symphonies. Fish \& shellfish immunology 25: 384-396.

20. Petit, J., and Wiegertjes, G. F. 2016. Long-lived effects of administering beta-glucans: Indications for trained immunity in fish. Dev Comp Immunol 64: 93-102.

21. Meena, D. K., Das, P., Kumar, S., Mandal, S. C., Prusty, A. K., Singh, S. K., Akhtar, M. S., Behera, B. K., Kumar, K., Pal, A. K., and Mukherjee, S. C. 2013. Beta-glucan: an ideal immunostimulant in aquaculture (a review). Fish physiology and biochemistry 39: 431-457.

22. Sinha, A. K., Kumar, V., Makkar, H. P. S., De Boeck, G., and Becker, K. 2011. Non-starch polysaccharides and their role in fish nutrition - A review. Food Chem 127: 1409-1426.

23. Ferreira, S. S., Passos, C. P., Madureira, P., Vilanova, M., and Coimbra, M. A. 2015. Structure-function relationships of immunostimulatory polysaccharides: A review. Carbohydrate polymers 132: 378-396.

24. Vetvicka, V., Vannucci, L., and Sima, P. 2013. The Effects of beta - Glucan on Fish Immunity. North American journal of medical sciences 5: 580-588.

25. Akira, S., Uematsu, S., and Takeuchi, O. 2006. Pathogen recognition and innate immunity. Cell 124: 783-801.

26. Gibson, G. R., Probert, H. M., Loo, J. V., Rastall, R. A., and Roberfroid, M. B. 2004. Dietary modulation of the human colonic microbiota: updating the concept of prebiotics. Nutr Res Rev 17: 259-275.

27. Brown, G. D., and Gordon, S. 2001. Immune recognition. A new receptor for beta-glucans. Nature 413: 36-37. 
28. Zelensky, A. N., and Gready, J. E. 2005. The C-type lectin-like domain superfamily. The FEBS journal 272: 6179-6217.

29. Ariizumi, K., Shen, G. L., Shikano, S., Xu, S., Ritter, R., 3rd, Kumamoto, T., Edelbaum, D., Morita, A., Bergstresser, P. R., and Takashima, A. 2000. Identification of a novel, dendritic cell-associated molecule, dectin-1, by subtractive cDNA cloning. The Journal of biological chemistry 275: 20157-20167.

30. Brown, G. D., Taylor, P. R., Reid, D. M., Willment, J. A., Williams, D. L., Martinez-Pomares, L., Wong, S. Y. C., and Gordon, S. 2002. Dectin-1 is a major beta-glucan receptor on macrophages. J Exp Med 196: 407-412.

31. Adachi, Y., Ishii, T., Ikeda, Y., Hoshino, A., Tamura, H., Aketagawa, J., Tanaka, S., and Ohno, N. 2004. Characterization of beta-glucan recognition site on C-type lectin, dectin 1. Infect Immun 72: 4159-4171.

32. Dulal, H. P., Adachi, Y., Ohno, N., and Yamaguchi, Y. 2018. beta-Glucan-induced cooperative oligomerization of Dectin-1 C-type lectin-like domain. Glycobiology 28: 612-623.

33. Brown, J., O'Callaghan, C. A., Marshall, A. S., Gilbert, R. J., Siebold, C., Gordon, S., Brown, G. D., and Jones, E. Y. 2007. Structure of the fungal beta-glucan-binding immune receptor dectin-1: implications for function. Protein science : a publication of the Protein Society 16: 1042-1052.

34. Kanagawa, M., Satoh, T., Ikeda, A., Adachi, Y., Ohno, N., and Yamaguchi, Y. 2011. Structural Insights into Recognition of Triple-helical beta-Glucans by an Insect Fungal Receptor. Journal of Biological Chemistry 286: 29158-29165.

35. Goodridge, H. S., Reyes, C. N., Becker, C. A., Katsumoto, T. R., Ma, J., Wolf, A. J., Bose, N., Chan, A. S. H., Magee, A. S., Danielson, M. E., Weiss, A., Vasilakos, J. P., and Underhill, D. M. 2011. Activation of the innate immune receptor Dectin-1 upon formation of a 'phagocytic synapse'. Nature 472: 471-U541.

36. Tang, J., Lin, G., Langdon, W. Y., Tao, L., and Zhang, J. 2018. Regulation of C-Type Lectin Receptor-Mediated Antifungal Immunity. Front Immunol 9: 123.

37. Wegener, E., and Krappmann, D. 2007. CARD-Bcl10-Malt1 signalosomes: missing link to NFkappaB. Science's STKE : signal transduction knowledge environment 2007: pe21.

38. Gross, O., Gewies, A., Finger, K., Schafer, M., Sparwasser, T., Peschel, C., Forster, I., and Ruland, J. 2006. Card9 controls a non-TLR signalling pathway for innate anti-fungal immunity. Nature 442: 651-656.

39. Plato, A., Hardison, S. E., and Brown, G. D. 2015. Pattern recognition receptors in antifungal immunity. Semin Immunopathol 37: 97-106.

40. Underhill, D. M., Rossnagle, E., Lowell, C. A., and Simmons, R. M. 2005. Dectin-1 activates Syk tyrosine kinase in a dynamic subset of macrophages for reactive oxygen production. Blood 106: 2543-2550.

41. Petit, J., Bailey, E. C., Wheeler, R. T., Ferreira de Oliveira, C. A., Forlenza, M., and Wiegertjes, G. F. 2019. Studies Into $\beta$-Glucan Recognition in Fish Suggests a Key Role for the C-Type Lectin Pathway. Front Immunol 10: 280.

42. Zelensky, A. N., and Gready, J. E. 2004. C-type lectin-like domains in Fugu rubripes. BMC genomics 5: 51.

43. Legentil, L., Paris, F., Ballet, C., Trouvelot, S., Daire, X., Vetvicka, V., and Ferrieres, V. 2015. Molecular Interactions of beta-(1 -> 3)-Glucans with Their Receptors. Molecules 20: 97459766.

44. Rogers, N. C., Slack, E. C., Edwards, A. D., Nolte, M. A., Schulz, O., Schweighoffer, E., Williams, D. L., Gordon, S., Tybulewicz, V. L., Brown, G. D., and Reis e Sousa, C. 2005. Sykdependent cytokine induction by Dectin-1 reveals a novel pattern recognition pathway for $\mathrm{C}$ type lectins. Immunity 22: 507-517.

45. Gantner, B. N., Simmons, R. M., Canavera, S. J., Akira, S., and Underhill, D. M. 2003. Collaborative induction of inflammatory responses by dectin-1 and toll-like receptor 2. J Exp Med 197: 1107-1117.

46. Soltanian, S., Francois, J. M., Dhont, J., Arnouts, S., Sorgeloos, P., and Bossier, P. 2007. Enhanced disease resistance in Artemia by application of commercial beta-glucans sources and chitin in a gnotobiotic Artemia challenge test. Fish \& shellfish immunology 23: 13041314.

47. Thornton, B. P., Vetvicka, V., Pitman, M., Goldman, R. C., and Ross, G. D. 1996. Analysis of the sugar specificity and molecular location of the beta-glucan-binding lectin site of complement receptor type 3 (CD11b/CD18). Journal of immunology 156: 1235-1246.

48. van Bruggen, R., Drewniak, A., Jansen, M., van Houdt, M., Roos, D., Chapel, H., Verhoeven, A. J., and Kuijpers, T. W. 2009. Complement receptor 3, not Dectin-1, is the major receptor on human neutrophils for beta-glucan-bearing particles. Molecular immunology 47: 575-581.

49. Vetvicka, V., Thornton, B. P., and Ross, G. D. 1996. Soluble beta-glucan polysaccharide binding to the lectin site of neutrophil or natural killer cell complement receptor type 3 
(CD11b/CD18) generates a primed state of the receptor capable of mediating cytotoxicity of iC3b-opsonized target cells. J Clin Invest 98: 50-61.

50. Zimmerman, J. W., Lindermuth, J., Fish, P. A., Palace, G. P., Stevenson, T. T., and DeMong, D. E. 1998. A novel carbohydrate-glycosphingolipid interaction between a beta-(1-3)-glucan immunomodulator, PGG-glucan, and lactosylceramide of human leukocytes. The Journal of biological chemistry 273: 22014-22020.

51. Hahn, P. Y., Evans, S. E., Kottom, T. J., Standing, J. E., Pagano, R. E., and Limper, A. H. 2003. Pneumocystis carinii cell wall beta-glucan induces release of macrophage inflammatory protein-2 from alveolar epithelial cells via a lactosylceramide-mediated mechanism. The Journal of biological chemistry 278: 2043-2050.

52. Dushkin, M. I., Safina, A. F., Vereschagin, E. I., and Schwartz, Y. 1996. Carboxymethylated beta-1,3-glucan inhibits the binding and degradation of acetylated low density lipoproteins in macrophages in vitro and modulates their plasma clearance in vivo. Cell biochemistry and function 14: 209-217.

53. Rice, P. J., Kelley, J. L., Kogan, G., Ensley, H. E., Kalbfleisch, J. H., Browder, I. W., and Williams, D. L. 2002. Human monocyte scavenger receptors are pattern recognition receptors for (1-->3)-beta-D-glucans. Journal of leukocyte biology 72: 140-146.

54. Vereschagin, E. I., van Lambalgen, A. A., Dushkin, M. I., Schwartz, Y. S., Polyakov, L., Heemskerk, A., Huisman, E., Thijs, L. G., and van den Bos, G. C. 1998. Soluble glucan protects against endotoxin shock in the rat: the role of the scavenger receptor. Shock 9: 193-198.

55. Jozefowski, S., Yang, Z., Marcinkiewicz, J., and Kobzik, L. 2012. Scavenger receptors and beta-glucan receptors participate in the recognition of yeasts by murine macrophages. Inflammation research : official journal of the European Histamine Research Society ... [et al.] 61: 113-126.

56. Netea, Mihai G., Quintin, J., and van der Meer, Jos W. M. 2011. Trained Immunity: A Memory for Innate Host Defense. Cell host \& microbe 9: 355-361.

57. Netea, M. G. 2013. Training innate immunity: the changing concept of immunological memory in innate host defence. European journal of clinical investigation 43: 881-884.

58. Quintin, J., Saeed, S., Martens, J. H. A., Giamarellos-Bourboulis, E. J., Ifrim, D. C., Logie, C., Jacobs, L., Jansen, T., Kullberg, B. J., Wijmenga, C., Joosten, L. A. B., Xavier, R. J., van der Meer, J. W. M., Stunnenberg, H. G., and Netea, M. G. 2012. Candida albicans infection affords protection against reinfection via functional reprogramming of monocytes. Cell host \& microbe 12: 223-232.

59. Arts, R. J., Novakovic, B., Ter Horst, R., Carvalho, A., Bekkering, S., Lachmandas, E., Rodrigues, F., Silvestre, R., Cheng, S. C., Wang, S. Y., Habibi, E., Goncalves, L. G., Mesquita, I., Cunha, C., van Laarhoven, A., van de Veerdonk, F. L., Williams, D. L., van der Meer, J. W., Logie, C., O'Neill, L. A., Dinarello, C. A., Riksen, N. P., van Crevel, R., Clish, C., Notebaart, R. A., Joosten, L. A., Stunnenberg, H. G., Xavier, R. J., and Netea, M. G. 2016. Glutaminolysis and Fumarate Accumulation Integrate Immunometabolic and Epigenetic Programs in Trained Immunity. Cell metabolism 24: 807-819.

60. Arts, R. J. W., Carvalho, A., La Rocca, C., Palma, C., Rodrigues, F., Silvestre, R., Kleinnijenhuis, J., Lachmandas, E., Goncalves, L. G., Belinha, A., Cunha, C., Oosting, M., Joosten, L. A. B., Matarese, G., van Crevel, R., and Netea, M. G. 2016. Immunometabolic Pathways in BCG-Induced Trained Immunity. Cell reports 17: 2562-2571.

61. Bekkering, S., Arts, R. J. W., Novakovic, B., Kourtzelis, I., van der Heijden, C. D. C. C., Li, Y., Popa, C. D., ter Horst, R., van Tuijl, J., Netea-Maier, R. T., de Veerdonk, F. L. V., Chavakis, T., Joosten, L. A. B., van der Meer, J. W. M., Stunnenberg, H., Riksen, N. P., and Netea, M. G. 2018. Metabolic Induction of Trained Immunity through the Mevalonate Pathway. Cell 172: $135-+$.

62. Cheng, S. C., Quintin, J., Cramer, R. A., Shepardson, K. M., Saeed, S., Kumar, V., Giamarellos-Bourboulis, E. J., Martens, J. H. A., Rao, N. A., Aghajanirefah, A., Manjeri, G. R., Li, Y., Ifrim, D. C., Arts, R. J. W., van der Meer, B. M. J. W., Deen, P. M. T., Logie, C., O'Neill, L. A., Willems, P., van de Veerdonk, F. L., van der Meer, J. W. M., Ng, A., Joosten, L. A. B., Wijmenga, C., Stunnenberg, H. G., Xavier, R. J., and Netea, M. G. 2014. mTORand HIF-1 alpha-mediated aerobic glycolysis as metabolic basis for trained immunity. Science 345: 1579-+.

63. Dominguez-Andres, J., Novakovic, B., Li, Y., Scicluna, B. P., Gresnigt, M. S., Arts, R. J. W., Oosting, M., Moorlag, S., Groh, L. A., Zwaag, J., Koch, R. M., Ter Horst, R., Joosten, L. A. B., Wijmenga, C., Michelucci, A., van der Poll, T., Kox, M., Pickkers, P., Kumar, V., Stunnenberg, H., and Netea, M. G. 2018. The Itaconate Pathway Is a Central Regulatory Node Linking Innate Immune Tolerance and Trained Immunity. Cell metabolism.

64. Saeed, S., Quintin, J., Kerstens, H. H., Rao, N. A., Aghajanirefah, A., Matarese, F., Cheng, S. C., Ratter, J., Berentsen, K., van der Ent, M. A., Sharifi, N., Janssen-Megens, E. M., Ter 
Huurne, M., Mandoli, A., van Schaik, T., Ng, A., Burden, F., Downes, K., Frontini, M., Kumar, V., Giamarellos-Bourboulis, E. J., Ouwehand, W. H., van der Meer, J. W., Joosten, L. A., Wijmenga, C., Martens, J. H., Xavier, R. J., Logie, C., Netea, M. G., and Stunnenberg, H. G. 2014. Epigenetic programming of monocyte-to-macrophage differentiation and trained innate immunity. Science 345: 1251086.

65. Kleinnijenhuis, J., Quintin, J., Preijers, F., Joosten, L. A., Ifrim, D. C., Saeed, S., Jacobs, C., van Loenhout, J., de Jong, D., Stunnenberg, H. G., Xavier, R. J., van der Meer, J. W., van Crevel, R., and Netea, M. G. 2012. Bacille Calmette-Guerin induces NOD2-dependent nonspecific protection from reinfection via epigenetic reprogramming of monocytes. Proceedings of the National Academy of Sciences of the United States of America 109: 1753717542.

66. Alvarez-Errico, D., Vento-Tormo, R., Sieweke, M., and Ballestar, E. 2015. Epigenetic control of myeloid cell differentiation, identity and function. Nature Reviews Immunology 15: 7-17.

67. Netea, M. G., Latz, E., Mills, K. H., and O'Neill, L. A. 2015. Innate immune memory: a paradigm shift in understanding host defense. Nat Immunol 16: 675-679.

68. Kurtz, J. 2005. Specific memory within innate immune systems. Trends Immunol 26: 186192.

69. Martens, E. C., Lowe, E. C., Chiang, H., Pudlo, N. A., Wu, M., McNulty, N. P., Abbott, D. W., Henrissat, B., Gilbert, H. J., Bolam, D. N., and Gordon, J. I. 2011. Recognition and degradation of plant cell wall polysaccharides by two human gut symbionts. PLoS biology 9: e1001221.

70. Temple, M. J., Cuskin, F., Basle, A., Hickey, N., Speciale, G., Williams, S. J., Gilbert, H. J., and Lowe, E. C. 2017. A Bacteroidetes locus dedicated to fungal 1,6-beta-glucan degradation: Unique substrate conformation drives specificity of the key endo-1,6-betaglucanase. The Journal of biological chemistry 292: 10639-10650.

71. Ulven, T. 2012. Short-chain free fatty acid receptors FFA2/GPR43 and FFA3/GPR41 as new potential therapeutic targets. Frontiers in endocrinology 3: 111.

72. Li, M., van Esch, B. C. A. M., Wagenaar, G. T. M., Garssen, J., Folkerts, G., and Henricks, P. A. J. 2018. Pro- and anti-inflammatory effects of short chain fatty acids on immune and endothelial cells. Eur J Pharmacol 831: 52-59.

73. Aoyama, M., Kotani, J., and Usami, M. 2010. Butyrate and propionate induced activated or non-activated neutrophil apoptosis via HDAC inhibitor activity but without activating GPR41/GPR-43 pathways. Nutrition 26: 653-661.

74. Immerstrand, T., Andersson, K. E., Wange, C., Rascon, A., Hellstrand, P., Nyman, M., Cui, S. W., Bergenstahl, B., Tragardh, C., and Oste, R. 2010. Effects of oat bran, processed to different molecular weights of beta-glucan, on plasma lipids and caecal formation of SCFA in mice. The British journal of nutrition 104: 364-373.

75. Lange, K., Hugenholtz, F., Jonathan, M. C., Schols, H. A., Kleerebezem, M., Smidt, H., Muller, M., and Hooiveld, G. J. 2015. Comparison of the effects of five dietary fibers on mucosal transcriptional profiles, and luminal microbiota composition and SCFA concentrations in murine colon. Molecular nutrition \& food research 59: 1590-1602.

76. Kawashima, T., Kosaka, A., Yan, H., Guo, Z., Uchiyama, R., Fukui, R., Kaneko, D., Kumagai, Y., You, D. J., Carreras, J., Uematsu, S., Jang, M. H., Takeuchi, O., Kaisho, T., Akira, S., Miyake, K., Tsutsui, H., Saito, T., Nishimura, I., and Tsuji, N. M. 2013. Double-stranded RNA of intestinal commensal but not pathogenic bacteria triggers production of protective interferon-beta. Immunity 38: 1187-1197.

77. Arena, M. P., Caggianiello, G., Fiocco, D., Russo, P., Torelli, M., Spano, G., and Capozzi, V. 2014. Barley beta-glucans-containing food enhances probiotic performances of beneficial bacteria. Int J Mol Sci 15: 3025-3039.

78. Adel, M., Lazado, C. C., Safari, R., Yeganeh, S., and Zorriehzahra, M. J. 2017. Aqualase (R), a yeast-based in-feed probiotic, modulates intestinal microbiota, immunity and growth of rainbow trout Oncorhynchus mykiss. Aquac Res 48: 1815-1826.

79. Kuhlwein, H., Emery, M. J., Rawling, M. D., Harper, G. M., Merrifield, D. L., and Davies, S. J. 2013. Effects of a dietary beta- $(1,3)(1,6)$-D-glucan supplementation on intestinal microbial communities and intestinal ultrastructure of mirror carp (Cyprinus carpio L.). J Appl Microbiol 115: $1091-1106$.

80. Jorgensen, J. B., Sharp, G. J. E., Secombes, C. J., and Robertsen, B. 1993. Effect of a YeastCell-Wall Glucan on the Bactericidal Activity of Rainbow-Trout Macrophages. Fish \& shellfish immunology 3: 267-277.

81. Chen, D., and Ainsworth, A. J. 1992. Glucan Administration Potentiates Immune DefenseMechanisms of Channel Catfish, Ictalurus-Punctatus Rafinesque. J Fish Dis 15: 295-304.

82. Jorgensen, J. B., and Robertsen, B. 1995. Yeast Beta-Glucan Stimulates Respiratory Burst Activity of Atlantic Salmon (Salmo-Salar L) Macrophages. Dev Comp Immunol 19: 43-57. 
83. Jeney, G., Galeotti, M., Volpatti, D., Jeney, Z., and Anderson, D. P. 1997. Prevention of stress in rainbow trout (Oncorhynchus mykiss) fed diets containing different doses of glucan. Aquaculture 154: 1-15.

84. Robertsen, B., Rørstad, G., Engstad, R., and Raa, J. 1990. Enhancement of non-specific disease resistance in Atlantic salmon, Salmo salar L., by a glucan from Saccharomyces cerevisiae cell walls. J Fish Dis 13: 391-400.

85. Siwicki, A. K., Anderson, D. P., and Rumsey, G. L. 1994. Dietary intake of immunostimulants by rainbow trout affects non-specific immunity and protection against furunculosis. Veterinary immunology and immunopathology 41: 125-139.

86. Goodridge, H. S., Wolf, A. J., and Underhill, D. M. 2009. Beta-glucan recognition by the innate immune system. Immunological reviews 230: 38-50.

87. Akramiene, D., Kondrotas, A., Didziapetriene, J., and Kevelaitis, E. 2007. Effects of betaglucans on the immune system. Med Lith 43: 597-606.

88. Raa, J. 2015. Immune modulation by non-digestible and non-absorbable beta-1,3/1,6glucan. Microb Ecol Health Dis 26: 27824.

89. Soltanian, S., Stuyven, E., Cox, E., Sorgeloos, P., and Bossier, P. 2009. Beta-glucans as immunostimulant in vertebrates and invertebrates. Crit Rev Microbiol 35: 109-138.

90. Swennen, K., Courtin, C. M., and Delcour, J. A. 2006. Non-digestible oligosaccharides with prebiotic properties. Critical reviews in food science and nutrition 46: 459-471.

91. Hino, S., Kito, A., Yokoshima, R., Sugino, R., Oshima, K., Morita, T., Okajima, T., Nadano, D., Uchida, K., and Matsuda, T. 2012. Discharge of solubilized and Dectin-1-reactive betaglucan from macrophage cells phagocytizing insoluble beta-glucan particles: involvement of reactive oxygen species (ROS)-driven degradation. Biochem Biophys Res Commun 421: 329334.

92. Dalmo, R. A., Ingebrigtsen, K., Horsberg, T. E., and Seljelid, R. 1994. Intestinal-Absorption of Immunomodulatory Laminaran and Derivatives in Atlantic Salmon, Salmo-Salar L. J Fish Dis 17: 579-589.

93. Lokka, G., Falk, K., Austbo, L., and Koppang, E. O. 2014. Uptake of yeast cells in the Atlantic salmon (Salmo salar L.) intestine. Dev Comp Immunol 47: 77-80.

94. Refstie, S., Baeverfjord, G., Seim, R. R., and Elvebo, O. 2010. Effects of dietary yeast cell wall beta-glucans and MOS on performance, gut health, and salmon lice resistance in Atlantic salmon (Salmo salar) fed sunflower and soybean meal. Aquaculture 305: 109-116.

95. Covello, J. M., Friend, S. E., Purcell, S. L., Burka, J. F., Markham, R. J. F., Donkin, A. W., Groman, D. B., and Fast, M. D. 2012. Effects of orally administered immunostimulants on inflammatory gene expression and sea lice (Lepeophtheirus salmonis) burdens on Atlantic salmon (Salmo salar). Aquaculture 366: 9-16.

96. Djordjevic, B., Skugor, S., Jorgensen, S. M., Overland, M., Mydland, L. T., and Krasnov, A. 2009. Modulation of splenic immune responses to bacterial lipopolysaccharide in rainbow trout (Oncorhynchus mykiss) fed lentinan, a beta-glucan from mushroom Lentinula edodes. Fish \& shellfish immunology 26: 201-209.

97. Lauridsen, J. H., and Buchmann, K. 2010. Effects of Short- and Long-Term Glucan Feeding of Rainbow Trout (Salmonidae) on the Susceptibility to Ichthyophthirius Multifiliis Infections. Acta Ichthyol Piscat 40: 61-66.

98. Skov, J., Kania, P. W., Holten-Andersen, L., Fouz, B., and Buchmann, K. 2012. Immunomodulatory effects of dietary beta-1,3-glucan from Euglena gracilis in rainbow trout (Oncorhynchus mykiss) immersion vaccinated against Yersinia ruckeri. Fish \& shellfish immunology 33: 111-120.

99. Schmitt, P., Wacyk, J., Morales-Lange, B., Rojas, V., Guzman, F., Dixon, B., and Mercado, L. 2015. Immunomodulatory effect of cathelicidins in response to a beta-glucan in intestinal epithelial cells from rainbow trout. Dev Comp Immunol 51: 160-169.

100. El-Boshy, M. E., El-Ashram, A. M., AbdelHamid, F. M., and Gadalla, H. A. 2010. Immunomodulatory effect of dietary Saccharomyces cerevisiae, beta-glucan and laminaran in mercuric chloride treated Nile tilapia (Oreochromis niloticus) and experimentally infected with Aeromonas hydrophila. Fish \& shellfish immunology 28: 802-808.

101. Guzman-Villanueva, L. T., Tovar-Ramirez, D., Gisbert, E., Cordero, H., Guardiola, F. A., Cuesta, A., Meseguer, J., Ascencio-Valle, F., and Esteban, M. A. 2014. Dietary administration of beta-1,3/1,6-glucan and probiotic strain Shewanella putrefaciens, single or combined, on gilthead seabream growth, immune responses and gene expression. Fish \& shellfish immunology 39: 34-41.

102. Dawood, M. A., Koshio, S., Ishikawa, M., and Yokoyama, S. 2015. Interaction effects of dietary supplementation of heat-killed Lactobacillus plantarum and beta-glucan on growth performance, digestibility and immune response of juvenile red sea bream, Pagrus major. Fish \& shellfish immunology 45: 33-42. 
103. Chang, C. S., Huang, S. L., Chen, S., and Chen, S. N. 2013. Innate immune responses and efficacy of using mushroom beta-glucan mixture (MBG) on orange-spotted grouper, Epinephelus coioides, aquaculture. Fish \& shellfish immunology 35: 115-125.

104. Pionnier, N., Falco, A., Miest, J. J., Shrive, A. K., and Hoole, D. 2014. Feeding common carp Cyprinus carpio with beta-glucan supplemented diet stimulates C-reactive protein and complement immune acute phase responses following PAMPs injection. Fish \& shellfish immunology 39: 285-295.

105. Gopalakannan, A., and Arul, V. 2010. Enhancement of the innate immune system and disease-resistant activity in Cyprinus carpio by oral administration of beta-glucan and whole cell yeast. Aquac Res 41: 884-892.

106. Lin, S. M., Pan, Y., Luo, L., and Luo, L. 2011. Effects of dietary beta-1,3-glucan, chitosan or raffinose on the growth, innate immunity and resistance of koi (Cyprinus carpio koi). Fish \& shellfish immunology 31: 788-794.

107. Pionnier, N., Falco, A., Miest, J., Frost, P., Irnazarow, I., Shrive, A., and Hoole, D. 2013. Dietary beta-glucan stimulate complement and C-reactive protein acute phase responses in common carp (Cyprinus carpio) during an Aeromonas salmonicida infection. Fish \& shellfish immunology 34: 819-831.

108. Falco, A., Frost, P., Miest, J., Pionnier, N., Irnazarow, I., and Hoole, D. 2012. Reduced inflammatory response to Aeromonas salmonicida infection in common carp (Cyprinus carpio L.) fed with beta-glucan supplements. Fish \& shellfish immunology 32: 1051-1057.

109. Miest, J. J., Falco, A., Pionnier, N. P. M., Frost, P., Irnazarow, I., Williams, G. T., and Hoole, D. 2012. The influence of dietary beta-glucan, PAMP exposure and Aeromonas salmonicida on apoptosis modulation in common carp (Cyprinus carpio). Fish \& shellfish immunology 33: 846-856.

110. van der Marel, M., Adamek, M., Gonzalez, S. F., Frost, P., Rombout, J. H. W. M., Wiegertjes, G. F., Savelkoul, H. F. J., and Steinhagen, D. 2012. Molecular cloning and expression of two beta-defensin and two mucin genes in common carp (Cyprinus carpio L.) and their upregulation after beta-glucan feeding. Fish \& shellfish immunology 32: 494-501.

111. Falco, A., Miest, J. J., Pionnier, N., Pietretti, D., Forlenza, M., Wiegertjes, G. F., and Hoole, D. 2014. beta-Glucan-supplemented diets increase poly(I:C)-induced gene expression of $M x$, possibly via TIr3-mediated recognition mechanism in common carp (Cyprinus carpio). Fish \& shellfish immunology 36: 494-502.

112. Miest, J. J., and Hoole, D. 2015. Time and concentration dependency of MacroGard(R) induced apoptosis. Fish \& shellfish immunology 42: 363-366.

113. Guselle, N. J., Speare, D. J., Markham, R. J. F., and Patelakis, S. 2010. Efficacy of Intraperitoneally and Orally Administered ProVale, a Yeast beta- $(1,3) /(1,6)$-D-glucan Product, in Inhibiting Xenoma Formation by the Microsporidian Loma salmonae on Rainbow Trout Gills. N Am J Aquacult 72: 65-72.

114. Paredes, M., Gonzalez, K., Figueroa, J., and Montiel-Eulefi, E. 2013. Immunomodulatory effect of prolactin on Atlantic salmon (Salmo salar) macrophage function. Fish physiology and biochemistry 39: 1215-1221.

115. Rodriguez, I., Chamorro, R., Novoa, B., and Figueras, A. 2009. beta-Glucan administration enhances disease resistance and some innate immune responses in zebrafish (Danio rerio). Fish \& shellfish immunology 27: 369-373.

116. Kiseleva, M., Balabanova, L., Elyakova, L., Rasskazov, V., and Zvyagintseva, T. 2014. Effect of treatment of chum salmon Oncorhynchus keta (Walbaum) eggs with 1,3;1,6-beta-Dglucans on their development and susceptibility to Saprolegnia infection. J Fish Dis 37: 3-10.

117. Zhang, Z., Swain, T., Bogwald, J., Dalmo, R. A., and Kumari, J. 2009. Bath immunostimulation of rainbow trout (Oncorhynchus mykiss) fry induces enhancement of inflammatory cytokine transcripts, while repeated bath induce no changes. Fish \& shellfish immunology 26: 677-684.

118. Selvaraj, V., Sampath, K., and Sekar, V. 2006. Adjuvant and immunostimulatory effects of beta-glucan administration in combination with lipopolysaccharide enhances survival and some immune parameters in carp challenged with Aeromonas hydrophila. Veterinary immunology and immunopathology 114: 15-24.

119. Przybylska-Diaz, D. A., Schmidt, J. G., Vera-Jimenez, N. I., Steinhagen, D., and Nielsen, M. E. 2013. beta-glucan enriched bath directly stimulates the wound healing process in common carp (Cyprinus carpio L.). Fish \& shellfish immunology 35: 998-1006.

120. Munang'andu, H. M., Mutoloki, S., and Evensen, O. 2015. A Review of the Immunological Mechanisms Following Mucosal Vaccination of Finfish. Front Immunol 6: 427.

121. Salinas, I. 2015. The Mucosal Immune System of Teleost Fish. Biology 4: 525-539.

122. Lam, K.-L., and Chi-Keung Cheung, P. 2013. Non-digestible long chain beta-glucans as novel prebiotics. Bioact Carbohydr Diet Fibre 2: 45-64. 
123. Wiegertjes, G. F., Wentzel, A. S., Spaink, H. P., Elks, P. M., and Fink, I. R. 2015. Polarization of immune responses in fish: The 'macrophages first' point of view. Molecular immunology.

124. Fredriksen, B. N., Saevareid, K., McAuley, L., Lane, M. E., Bogwald, J., and Dalmo, R. A. 2011. Early immune responses in Atlantic salmon (Salmo salar L.) after immunization with PLGA nanoparticles loaded with a model antigen and beta-glucan. Vaccine 29: 8338-8349.

125. Kudrenko, B., Snape, N., and Barnes, A. C. 2009. Linear and branched B(1-3) d-glucans activate but do not prime teleost macrophages in vitro and are inactivated by dilute acid: Implications for dietary immunostimulation. Fish \& shellfish immunology 26: 443-450.

126. Jung-Schroers, V., Adamek, M., Jung, A., Harris, S., Doza, O. S., Baumer, A., and Steinhagen, D. 2016. Feeding of beta-1,3/1,6-glucan increases the diversity of the intestinal microflora of carp (Cyprinus carpio). Aquacult Nutr 22: 1026-1039.

127. Carda-Dieguez, M., Mira, A., and Fouz, B. 2014. Pyrosequencing survey of intestinal microbiota diversity in cultured sea bass (Dicentrarchus labrax) fed functional diets. FEMS microbiology ecology 87: 451-459.

128. Gomez, D., Sunyer, J. O., and Salinas, I. 2013. The mucosal immune system of fish: The evolution of tolerating commensals while fighting pathogens. Fish \& shellfish immunology 35: 1729-1739.

129. Mikrou, A., Marioli, D., Papanastasiou, A. D., and Zarkadis, I. K. 2009. CR3 complement receptor: Cloning and characterization in rainbow trout. Fish \& shellfish immunology 26: 1928.

130. Nakao, M., Fujiki, K., Kondo, M., and Yano, T. 2003. Detection of complement receptors on head kidney phagocytes of the common carp Cyprinus carpio. Fisheries Sci 69: 929-935.

131. Akula, S., Mohammadamin, S., and Hellman, L. 2014. Fc Receptors for Immunoglobulins and Their Appearance during Vertebrate Evolution. Plos One 9.

132. Benard, E. L., Roobol, S. J., Spaink, H. P., and Meijer, A. H. 2014. Phagocytosis of mycobacteria by zebrafish macrophages is dependent on the scavenger receptor Marco, a key control factor of pro-inflammatory signalling. Dev Comp Immunol 47: 223-233.

133. Fink, I. R., Benard, E. L., Hermsen, T., Meijer, A. H., Forlenza, M., and Wiegertjes, G. F. 2015. Molecular and functional characterization of the scavenger receptor CD36 in zebrafish and common carp. Molecular immunology 63: 381-393.

134. Pietretti, D., and Wiegertjes, G. F. 2014. Ligand specificities of Toll-like receptors in fish: indications from infection studies. Dev Comp Immunol 43: 205-222.

135. Sattler, S., Ghadially, H., and Hofer, E. 2012. Evolution of the C-Type Lectin-Like Receptor Genes of the DECTIN-1 Cluster in the NK Gene Complex. Sci World J: 1-11.

136. Brogden, G., Krimmling, T., Adamek, M., Naim, H. Y., Steinhagen, D., and von KockritzBlickwede, M. 2014. The effect of beta-glucan on formation and functionality of neutrophil extracellular traps in carp (Cyprinus carpio L.). Dev Comp Immunol 44: 280-285.

137. Brogden, G., von Köckritz-Blickwede, M., Adamek, M., Reuner, F., Jung-Schroers, V., Naim, H. Y., and Steinhagen, D. 2012. $\beta$-Glucan protects neutrophil extracellular traps against degradation by Aeromonas hydrophila in carp (Cyprinus carpio). Fish \& shellfish immunology 33: $1060-1064$.

138. Pietretti, D., Vera-Jimenez, N. I., Hoole, D., and Wiegertjes, G. F. 2013. Oxidative burst and nitric oxide responses in carp macrophages induced by zymosan, MacroGard((R)) and selective dectin-1 agonists suggest recognition by multiple pattern recognition receptors. Fish \& shellfish immunology 35: 847-857.

139. Janeway, C. A., Jr., and Medzhitov, R. 2002. Innate immune recognition. Annual review of immunology 20: 197-216.

140. Marakalala, M. J., Williams, D. L., Hoving, J. C., Engstad, R., Netea, M. G., and Brown, G. D. 2013. Dectin-1 plays a redundant role in the immunomodulatory activities of beta-glucanrich ligands in vivo. Microbes and infection 15: 511-515.

141. Leentjens, J., Quintin, J., Gerretsen, J., Kox, M., Pickkers, P., and Netea, M. G. 2014. The Effects of Orally Administered Beta-Glucan on Innate Immune Responses in Humans, a Randomized Open-Label Intervention Pilot-Study. Plos One 9.

142. Kleinnijenhuis, J., Quintin, J., Preijers, F., Benn, C. S., Joosten, L. A. B., Jacobs, C., van Loenhout, J., Xavier, R. J., Aaby, P., van der Meer, J. W. M., van Crevel, R., and Netea, M. G. 2014. Long-Lasting Effects of BCG Vaccination on Both Heterologous Th1/Th17 Responses and Innate Trained Immunity. Journal of innate immunity 6: 152-158.

143. Ryals, J. A., Neuenschwander, U. H., Willits, M. G., Molina, A., Steiner, H. Y., and Hunt, M. D. 1996. Systemic acquired resistance. Plant Cell 8: 1809-1819.

144. Conrath, U. 2006. Systemic acquired resistance. Plant Signal Behav 1: 179-184.

145. Vernooij, B., Friedrich, L., Ahl Goy, P., Staub, T., Kessmann, H., and Ryals, J. 1995. 2, 6Dichloroisonicotinic acid-induced resistance to pathogens without the accumulation of salicylic acid. Mol Plant Microbe Interact 8: 228-234. 
146. Magor, B. G., and Magor, K. E. 2001. Evolution of effectors and receptors of innate immunity. Dev Comp Immunol 25: 651-682.

147. Magnadottir, B. 2006. Innate immunity of fish (overview). Fish \& shellfish immunology 20: 137-151.

148. Olivier, G., Eaton, C. A., and Campbell, N. 1986. Interaction between Aeromonas salmonicida and peritoneal macrophages of brook trout (Salvelinus fontinalis). Veterinary immunology and immunopathology 12: 223-234.

149. Kato, G., Kondo, H., Aoki, T., and Hirono, I. 2010. BCG vaccine confers adaptive immunity against Mycobacterium sp. infection in fish. Dev Comp Immunol 34: 133-140.

150. Kato, G., Kato, K., Saito, K., Pe, Y., Kondo, H., Aoki, T., and Hirono, I. 2011. Vaccine efficacy of Mycobacterium bovis BCG against Mycobacterium sp. infection in amberjack Seriola dumerili. Fish \& shellfish immunology 30: 467-472.

151. Kato, G., Kondo, H., Aoki, T., and Hirono, I. 2012. Mycobacterium bovis BCG vaccine induces non-specific immune responses in Japanese flounder against Nocardia seriolae. Fish \& shellfish immunology 33: 243-250.

152. Hohn, C., and Petrie-Hanson, L. 2012. Rag1-/- mutant zebrafish demonstrate specific protection following bacterial re-exposure. Plos One 7: e44451.

153. Kawakami, H., Shinohara, N., and Sakai, M. 1998. The non-specific immunostimulation and adjuvant effects of Vibrio anguillarum bacterin, M-glucan, chitin and Freund's complete adjuvant against Pasteurella piscicida infection in yellowtail. Fish Pathol 33: 287-292.

154. Samuel, M., Lam, T. J., and Sin, Y. M. 1996. Effect of Laminarin [beta(1,3)-D-Glucan] on the protective immunity of blue gourami, Trichogaster trichopterus against Aeromonas hydrophila. Fish \& shellfish immunology 6: 443-454.

155. Verlhac, V., Obach, A., Gabaudan, J., Schuep, W., and Hole, R. 1998. Immunomodulation by dietary vitamin $\mathrm{C}$ and glucan in rainbow trout (Oncorhynchus mykiss). Fish \& shellfish immunology 8: 409-424.

156. Bagni, M., Archetti, L., Amadori, M., and Marino, G. 2000. Effect of long-term oral administration of an immunostimulant diet on innate immunity in sea bass (Dicentrarchus labrax). J Vet Med B 47: 745-751.

157. Garcia-Valtanen, P., Martinez-Lopez, A., Lopez-Munoz, A., Bello-Perez, M., Medina-Gali, R. M., Ortega-Villaizan, M. D., Varela, M., Figueras, A., Mulero, V., Novoa, B., Estepa, A., and Coll, J. 2017. Zebra Fish Lacking Adaptive Immunity Acquire an Antiviral Alert State Characterized by Upregulated Gene Expression of Apoptosis, Multigene Families, and Interferon-Related Genes. Front Immunol 8: 121.

158. Alvarez-Rodriguez, M., Pereiro, P., Reyes-Lopez, F. E., Tort, L., Figueras, A., and Novoa, B. 2018. Analysis of the Long-Lived Responses Induced by Immunostimulants and Their Effects on a Viral Infection in Zebrafish (Danio rerio). Front Immunol 9.

159. Rebl, A., and Goldammer, T. 2018. Under control: The innate immunity of fish from the inhibitors' perspective. Fish \& shellfish immunology 77: 328-349.

160. Tokunaga, Y., Shirouzu, M., Sugahara, R., Yoshiura, Y., Kiryu, I., Ototake, M., Nagasawa, T., Somamoto, T., and Nakao, M. 2017. Comprehensive validation of T- and B-cell deficiency in rag1-null zebrafish: Implication for the robust innate defense mechanisms of teleosts. Scientific reports 7: 7536 .

161. Aoki, T., Takano, T., Santos, M. D., Kondo, H., and Hirono, I. 2008. Molecular innate immunity in teleost fish: review and future perspectives. In Fisheries for Global Welfare and Environment, Memorial Book of the 5th World Fisheries Congress. Terrapub: Tokyo, Japan. 263-276.

162. Wcisel, D. J., and Yoder, J. A. 2016. The confounding complexity of innate immune receptors within and between teleost species. Fish \& shellfish immunology 53: 24-34.

163. Zhang, Z., Chi, H., and Dalmo, R. A. 2019. Trained Innate Immunity of Fish Is a Viable Approach in Larval Aquaculture. Front Immunol 10.

164. Joerink, M., Ribeiro, C. M., Stet, R. J., Hermsen, T., Savelkoul, H. F., and Wiegertjes, G. F. 2006. Head kidney-derived macrophages of common carp (Cyprinus carpio L.) show plasticity and functional polarization upon differential stimulation. Journal of immunology 177: 61-69.

165. Maharana, J., Sahoo, B. R., Bej, A., Jena, I., Parida, A., Sahoo, J. R., Dehury, B., Patra, M. C., Martha, S. R., Balabantray, S., Pradhan, S. K., and Behera, B. K. 2015. Structural Models of Zebrafish (Danio rerio) NOD1 and NOD2 NACHT Domains Suggest Differential ATP Binding Orientations: Insights from Computational Modeling, Docking and Molecular Dynamics Simulations. Plos One 10.

166. Oehlers, S. H., Flores, M. V., Hall, C. J., Swift, S., Crosier, K. E., and Crosier, P. S. 2011. The inflammatory bowel disease (IBD) susceptibility genes NOD1 and NOD2 have conserved antibacterial roles in zebrafish. Dis Model Mech 4: 832-841. 
167. Zou, P. F., Chang, M. X., Li, Y., Xue, N. N., Li, J. H., Chen, S. N., and Nie, P. 2016. NOD2 in zebrafish functions in antibacterial and also antiviral responses via NF-kappa B, and also MDA5, RIG-I and MAVS. Fish \& shellfish immunology 55: 173-185.

168. Bekkering, S., Blok, B. A., Joosten, L. A., Riksen, N. P., van Crevel, R., and Netea, M. G. 2016. In Vitro Experimental Model of Trained Innate Immunity in Human Primary Monocytes. Clinical and vaccine immunology : CVI 23: 926-933.

169. Irnazarow, I. 1995. Genetic-Variability of Polish and Hungarian Carp Lines. Aquaculture 129: 215-215.

170. Piazzon, M. C., Savelkoul, H. F. J., Pietretti, D., Wiegertjes, G. F., and Forlenza, M. 2015. Carp Il10 Has Anti-Inflammatory Activities on Phagocytes, Promotes Proliferation of Memory T Cells, and Regulates B Cell Differentiation and Antibody Secretion. Journal of immunology 194: 187-199.

171. Saeij, J. P., Van Muiswinkel, W. B., Groeneveld, A., and Wiegertjes, G. F. 2002. Immune modulation by fish kinetoplastid parasites: a role for nitric oxide. Parasitology 124: 77-86.

172. Li, J., Barreda, D. R., Zhang, Y. A., Boshra, H., Gelman, A. E., LaPatra, S., Tort, L., and Sunyer, J. O. 2006. B lymphocytes from early vertebrates have potent phagocytic and microbicidal abilities. Nat Immunol 7: 1116-1124.

173. Pfaffl, M. W. 2001. A new mathematical model for relative quantification in real-time RT-PCR. Nucleic acids research 29: e45.

174. Oksanen, K. E., Halfpenny, N. J., Sherwood, E., Harjula, S. K., Hammaren, M. M., Ahava, M. J., Pajula, E. T., Lahtinen, M. J., Parikka, M., and Ramet, M. 2013. An adult zebrafish model for preclinical tuberculosis vaccine development. Vaccine 31: 5202-5209.

175. Medina-Gali, R. M., Ortega-Villaizan, M. D. M., Mercado, L., Novoa, B., Coll, J., and Perez, L. 2018. Beta-glucan enhances the response to SVCV infection in zebrafish. Dev Comp Immunol 84: 307-314.

176. Kleinnijenhuis, J., Quintin, J., Preijers, F., Joosten, L. A., Jacobs, C., Xavier, R. J., van der Meer, J. W., van Crevel, R., and Netea, M. G. 2014. BCG-induced trained immunity in NK cells: Role for non-specific protection to infection. Clinical immunology 155: 213-219.

177. Sun, J. C., Beilke, J. N., and Lanier, L. L. 2009. Adaptive immune features of natural killer cells. Nature 457: 557-561.

178. Schlums, H., Cichocki, F., Tesi, B., Theorell, J., Beziat, V., Holmes, T. D., Han, H., Chiang, S. C., Foley, B., Mattsson, K., Larsson, S., Schaffer, M., Malmberg, K. J., Ljunggren, H. G., Miller, J. S., and Bryceson, Y. T. 2015. Cytomegalovirus infection drives adaptive epigenetic diversification of NK cells with altered signaling and effector function. Immunity 42: 443456.

179. Magnadottir, B. 2010. Immunological control of fish diseases. Marine biotechnology 12: 361379.

180. Bowden, T. J. 2008. Modulation of the immune system of fish by their environment. Fish \& shellfish immunology 25: 373-383.

181. Dominguez-Andres, J., and Netea, M. G. 2018. Long-term reprogramming of the innate immune system. Journal of leukocyte biology.

182. Medina-Gali, R., Bello-Perez, M., Martinez-Lopez, A., Falco, A., Ortega-Villaizan, M. M., Encinar, J. A., Novoa, B., Coll, J., and Perez, L. 2018. Chromatin immunoprecipitation and high throughput sequencing of SVCV-infected zebrafish reveals novel epigenetic histone methylation patterns involved in antiviral immune response. Fish \& shellfish immunology 82: 514-521.

183. Best, C., Ikert, H., Kostyniuk, D. J., Craig, P. M., Navarro-Martin, L., Marandel, L., and Mennigen, J. A. 2018. Epigenetics in teleost fish: From molecular mechanisms to physiological phenotypes. Comp Biochem Phys B 224: 210-244.

184. Cerenius, L., Kawabata, S.-i., Söderhäll, K., Bacic, A., Fincher, G. B., and Stone, B. A. 2009. Chapter 4.5.1 - Biological and Immunological Aspects of Innate Defence Mechanisms Activated by $(1,3)-\beta$-Glucans and Related Polysaccharides in Invertebrates. In Chemistry, Biochemistry, and Biology of 1-3 Beta Glucans and Related Polysaccharides. Academic Press, San Diego. 563-577.

185. Brown, G. D., Williams, D. L., Bacic, A., Fincher, G. B., and Stone, B. A. 2009. Chapter 4.5.2 - $(1,3)-\beta-G l u c a n s$ in Innate Immunity: Mammalian Systems. In Chemistry, Biochemistry, and Biology of 1-3 Beta Glucans and Related Polysaccharides. Academic Press, San Diego. 579619.

186. Petrie, A. G., and Ellis, A. E. 2006. Evidence of particulate uptake by the gut of Atlantic salmon (Salmo salar L.). Fish \& shellfish immunology 20: 660-664.

187. Rombout, J. H. W. M., Tavernethiele, A. J., and Villena, M. I. 1993. The Gut-Associated Lymphoid-Tissue (Galt) of Carp (Cyprinus-Carpio L) - an Immunocytochemical Analysis. Dev Comp Immunol 17: 55-66. 
188. Round, J. L., and Mazmanian, S. K. 2009. The gut microbiota shapes intestinal immune responses during health and disease. Nature reviews. Immunology 9: 313-323.

189. Purchiaroni, F., Tortora, A., Gabrielli, M., Bertucci, F., Gigante, G., Ianiro, G., Ojetti, V., Scarpellini, E., and Gasbarrini, A. 2013. The role of intestinal microbiota and the immune system. European review for medical and pharmacological sciences 17: 323-333.

190. Belkaid, Y., and Hand, T. W. 2014. Role of the microbiota in immunity and inflammation. Cell 157: $121-141$.

191. Rurangwa, E., Laranja, J. L., Van Houdt, R., Delaedt, Y., Geraylou, Z., Van de Wiele, T., Van Loo, J., Van Craeyveld, V., Courtin, C. M., Delcour, J. A., and Ollevier, F. 2009. Selected nondigestible carbohydrates and prebiotics support the growth of probiotic fish bacteria mono-cultures in vitro. J Appl Microbiol 106: 932-940.

192. Kihara, M., and Sakata, T. 2002. Production of short-chain fatty acids and gas from various oligosaccharides by gut microbes of carp (Cyprinus carpio L.) in micro-scale batch culture. Comparative Biochemistry and Physiology a-Molecular and Integrative Physiology 132: 333340.

193. Kihara, M., and Sakata, T. 1997. Fermentation of dietary carbohydrates to short-chain fatty acids by gut microbes and its influence on intestinal morphology of a detritivorous teleost Tilapia (Oreochromis niloticus). Comp Biochem Phys A 118: 1201-1207.

194. Leenhouwers, J. I., Pellikaan, W. F., Huizing, H. F. A., Coolen, R. O. M., Verreth, J. A. J., and Schrama, J. W. 2008. Fermentability of carbohydrates in an in vitro batch culture method using inocula from Nile tilapia (Oreochromis niloticus) and European sea bass (Dicentrarchus labrax). Aquacult Nutr 14: 523-532.

195. Nikkhoo, M., Yousefian, M., Safari, R., and Nikkhoo, M. 2010. The influence probiotic of Aqualase on the survival, growth, intestinal microflora and challenge infection in wild carp (Cyprinus carpio L.). Research Journal of Fisheries and Hydrobiology 5: 168-172.

196. Perez-Jaramillo, J. E., Carrion, V. J., Bosse, M., Ferrao, L. F. V., de Hollander, M., Garcia, A. A. F., Ramirez, C. A., Mendes, R., and Raaijmakers, J. M. 2017. Linking rhizosphere microbiome composition of wild and domesticated Phaseolus vulgaris to genotypic and root phenotypic traits. The ISME journal 11: 2244-2257.

197. Masella, A. P., Bartram, A. K., Truszkowski, J. M., Brown, D. G., and Neufeld, J. D. 2012. PANDAseq: paired-end assembler for illumina sequences. BMC bioinformatics 13: 31.

198. Cole, J. R., Wang, Q., Fish, J. A., Chai, B., McGarrell, D. M., Sun, Y., Brown, C. T., PorrasAlfaro, A., Kuske, C. R., and Tiedje, J. M. 2014. Ribosomal Database Project: data and tools for high throughput rRNA analysis. Nucleic acids research 42: D633-642.

199. Dodt, M., Roehr, J. T., Ahmed, R., and Dieterich, C. 2012. FLEXBAR-Flexible Barcode and Adapter Processing for Next-Generation Sequencing Platforms. Biology 1: 895-905.

200. Edgar, R. C. 2010. Search and clustering orders of magnitude faster than BLAST. Bioinformatics 26: 2460-2461.

201. Rognes, T., Flouri, T., Nichols, B., Quince, C., and Mahe, F. 2016. VSEARCH: a versatile open source tool for metagenomics. Peerj 4.

202. Edgar, R. C., Haas, B. J., Clemente, J. C., Quince, C., and Knight, R. 2011. UCHIME improves sensitivity and speed of chimera detection. Bioinformatics 27: 2194-2200.

203. McDonald, D., Clemente, J. C., Kuczynski, J., Rideout, J. R., Stombaugh, J., Wendel, D., Wilke, A., Huse, S., Hufnagle, J., Meyer, F., Knight, R., and Caporaso, J. G. 2012. The Biological Observation Matrix (BIOM) format or: how I learned to stop worrying and love the ome-ome. Gigascience 1.

204. Wang, Q., Garrity, G. M., Tiedje, J. M., and Cole, J. R. 2007. Naive Bayesian classifier for rapid assignment of rRNA sequences into the new bacterial taxonomy. Appl Environ Microb 73: 5261-5267.

205. Koster, J., and Rahmann, S. 2012. Snakemake--a scalable bioinformatics workflow engine. Bioinformatics 28: 2520-2522.

206. Kuczynski, J., Stombaugh, J., Walters, W. A., Gonzalez, A., Caporaso, J. G., and Knight, R. 2011. Using QIIME to analyze 16S rRNA gene sequences from microbial communities. Current protocols in bioinformatics Chapter 10: Unit 10.17.

207. Paulson, J. N., Pop, M., and Bravo, H. 2013. metagenomeSeq: Statistical analysis for sparse high-throughput sequencing. Bioconductor package 1.

208. Team, R. C. 2013. R: A language and environment for statistical computing.

209. Paulson, J. N., Stine, O. C., Bravo, H. C., and Pop, M. 2013. Differential abundance analysis for microbial marker-gene surveys. Nature methods 10: 1200-1202.

210. McMurdie, P. J., and Holmes, S. 2013. phyloseq: an R package for reproducible interactive analysis and graphics of microbiome census data. Plos One 8: e61217.

211. Kursa, M. B., Jankowski, A., and Rudnicki, W. R. 2010. Boruta - A System for Feature Selection. Fund Inform 101: 271-286.

212. Kolde, R. 2012. Pheatmap: pretty heatmaps. $R$ package version 61. 
213. Davies, Z. S., Mason, D., Brooks, A. E., Griffith, G. W., Merry, R. J., and Theodorou, M. K. 2000. An automated system for measuring gas production from forages inoculated with rumen fluid and its use in determining the effect of enzymes on grass silage. Anim Feed Sci Tech 83: 205-221.

214. Coles, L. T., Moughan, P. J., and Darragh, A. J. 2005. In vitro digestion and fermentation methods, including gas production techniques, as applied to nutritive evaluation of foods in the hindgut of humans and other simple-stomached animals. Anim Feed Sci Tech 123: 421444.

215. Williams, B. A., Verstegen, M. W. A., and Tamminga, S. 2001. Fermentation in the large intestine of single-stomached animals and its relationship to animal health. Nutr Res Rev 14: 207-227.

216. Williams, B. A., Bosch, M. W., Boer, H., Verstegen, M. W. A., and Tamminga, S. 2005. An in vitro batch culture method to assess potential fermentability of feed ingredients for monogastric diets. Anim Feed Sci Tech 123: 445-462.

217. Cone, J. W., vanGelder, A. H., Visscher, G. J. W., and Oudshoorn, L. 1996. Influence of rumen fluid and substrate concentration on fermentation kinetics measured with a fully automated time related gas production apparatus. Anim Feed Sci Tech 61: 113-128.

218. Groot, J. C. J., Cone, J. W., Williams, B. A., Debersaques, F. M. A., and Lantinga, E. A. 1996. Multiphasic analysis of gas production kinetics for in vitro fermentation of ruminant feeds. Anim Feed Sci Tech 64: 77-89.

219. Bauer, E., Williams, B. A., Voigt, C., Mosenthin, R., and Verstegen, M. W. A. 2001. Microbial activities of faeces from unweaned and adult pigs, in relation to selected fermentable carbohydrates. Anim Sci 73: 313-322.

220. Vissers, A. M., Pellikaan, W. F., Bouwhuis, A., Vincken, J. P., Gruppen, H., and Hendriks, W. H. 2018. Laminaria digitata phlorotannins decrease protein degradation and methanogenesis during in vitro ruminal fermentation. J Sci Food Agr 98: 3644-3650.

221. Nogales, B., Moore, E. R. B., Llobet-Brossa, E., Rossello-Mora, R., Amann, R., and Timmis, K. N. 2001. Combined use of $16 S$ ribosomal DNA and $16 S$ rRNA to study the bacterial community of polychlorinated biphenyl-polluted soil. Appl Environ Microb 67: 1874-1884.

222. Rooks, M. G., and Garrett, W. S. 2016. Gut microbiota, metabolites and host immunity. Nature reviews. Immunology 16: 341-352.

223. van Kessel, M. A., Dutilh, B. E., Neveling, K., Kwint, M. P., Veltman, J. A., Flik, G., Jetten, M. S., Klaren, P. H., and Op den Camp, H. J. 2011. Pyrosequencing of 16S rRNA gene amplicons to study the microbiota in the gastrointestinal tract of carp (Cyprinus carpio L.). AMB Express 1: 41.

224. Li, T., Long, M., Gatesoupe, F. J., Zhang, Q., Li, A., and Gong, X. 2015. Comparative analysis of the intestinal bacterial communities in different species of carp by pyrosequencing. Microbial ecology 69: 25-36.

225. Liu, H., Guo, X., Gooneratne, R., Lai, R., Zeng, C., Zhan, F., and Wang, W. 2016. The gut microbiome and degradation enzyme activity of wild freshwater fishes influenced by their trophic levels. Scientific reports 6: 24340.

226. Roeselers, G., Mittge, E. K., Stephens, W. Z., Parichy, D. M., Cavanaugh, C. M., Guillemin, K., and Rawls, J. F. 2011. Evidence for a core gut microbiota in the zebrafish. The ISME journal 5: 1595-1608.

227. Ndeh, D., Rogowski, A., Cartmell, A., Luis, A. S., Basle, A., Gray, J., Venditto, I., Briggs, J., Zhang, X., Labourel, A., Terrapon, N., Buffetto, F., Nepogodiev, S., Xiao, Y., Field, R. A., Zhu, Y., O'Neil, M. A., Urbanowicz, B. R., York, W. S., Davies, G. J., Abbott, D. W., Ralet, M. C., Martens, E. C., Henrissat, B., and Gilbert, H. J. 2017. Complex pectin metabolism by gut bacteria reveals novel catalytic functions. Nature 544: 65-70.

228. Xu, J., Mahowald, M. A., Ley, R. E., Lozupone, C. A., Hamady, M., Martens, E. C., Henrissat, B., Coutinho, P. M., Minx, P., Latreille, P., Cordum, H., Van Brunt, A., Kim, K., Fulton, R. S., Fulton, L. A., Clifton, S. W., Wilson, R. K., Knight, R. D., and Gordon, J. I. 2007. Evolution of symbiotic bacteria in the distal human intestine. PLoS biology 5: e156.

229. Martens, E. C., Chiang, H. C., and Gordon, J. I. 2008. Mucosal glycan foraging enhances fitness and transmission of a saccharolytic human gut bacterial symbiont. Cell host \& microbe 4: 447-457.

230. Degnan, B. A., and Macfarlane, G. T. 1995. Carbohydrate utilization patterns and substrate preferences in Bacteroides thetaiotaomicron. Anaerobe 1: 25-33.

231. Trompette, A., Gollwitzer, E. S., Yadava, K., Sichelstiel, A. K., Sprenger, N., Ngom-Bru, C., Blanchard, C., Junt, T., Nicod, L. P., Harris, N. L., and Marsland, B. J. 2014. Gut microbiota metabolism of dietary fiber influences allergic airway disease and hematopoiesis. Nature medicine 20: 159-166. 
232. Halnes, I., Baines, K. J., Berthon, B. S., MacDonald-Wicks, L. K., Gibson, P. G., and Wood, L. G. 2017. Soluble Fibre Meal Challenge Reduces Airway Inflammation and Expression of GPR43 and GPR41 in Asthma. Nutrients 9.

233. Kim, Y. S., Ke, F., and Zhang, Q. Y. 2009. Effect of beta-glucan on activity of antioxidant enzymes and $M x$ gene expression in virus infected grass carp. Fish \& shellfish immunology 27: 336-340.

234. Masui, R., Sasaki, M., Funaki, Y., Ogasawara, N., Mizuno, M., Iida, A., Izawa, S., Kondo, Y., Ito, Y., Tamura, Y., Yanamoto, K., Noda, H., Tanabe, A., Okaniwa, N., Yamaguchi, Y., Iwamoto, T., and Kasugai, K. 2013. G Protein-Coupled Receptor 43 Moderates Gut Inflammation Through Cytokine Regulation from Mononuclear Cells. Inflamm Bowel Dis 19: 2848-2856.

235. Ohira, H., Fujioka, Y., Katagiri, C., Mamoto, R., Aoyama-Ishikawa, M., Amako, K., Izumi, Y., Nishiumi, S., Yoshida, M., Usami, M., and Ikeda, M. 2013. Butyrate attenuates inflammation and lipolysis generated by the interaction of adipocytes and macrophages. Journal of atherosclerosis and thrombosis 20: 425-442.

236. Li, M., van Esch, B., Henricks, P. A. J., Garssen, J., and Folkerts, G. 2018. Time and Concentration Dependent Effects of Short Chain Fatty Acids on Lipopolysaccharide- or Tumor Necrosis Factor alpha-Induced Endothelial Activation. Frontiers in pharmacology 9: 233.

237. Voltolini, C., Battersby, S., Etherington, S. L., Petraglia, F., Norman, J. E., and Jabbour, H. N. 2012. A novel antiinflammatory role for the short-chain fatty acids in human labor. Endocrinology 153: 395-403.

238. Yang, L., Sado, T., Vincent Hirt, M., Pasco-Viel, E., Arunachalam, M., Li, J., Wang, X., Freyhof, J., Saitoh, K., Simons, A. M., Miya, M., He, S., and Mayden, R. L. 2015. Phylogeny and polyploidy: resolving the classification of cyprinine fishes (Teleostei: Cypriniformes). Mol Phylogenet Evol 85: 97-116.

239. Dubinska-Magiera, M., Daczewska, M., Lewicka, A., Migocka-Patrzalek, M., NiedbalskaTarnowska, J., and Jagla, K. 2016. Zebrafish: A model for the study of toxicants affecting muscle development and function. Int J Mol Sci 17.

240. Brugman, S. 2016. The zebrafish as a model to study intestinal inflammation. Dev Comp Immunol 64: 82-92.

241. MacRae, C. A., and Peterson, R. T. 2015. Zebrafish as tools for drug discovery. Nat Rev Drug Discov 14: 721-731.

242. Benard, E. L., Rougeot, J., Racz, P. I., Spaink, H. P., and Meijer, A. H. 2016. Transcriptomic approaches in the zebrafish model for tuberculosis-insights into host- and pathogen-specific determinants of the innate immune response. Adv Genet 95: 217-251.

243. Aparicio, S., Chapman, J., Stupka, E., Putnam, N., Chia, J. M., Dehal, P., Christoffels, A., Rash, S., Hoon, S., Smit, A., Gelpke, M. D., Roach, J., Oh, T., Ho, I. Y., Wong, M., Detter, C., Verhoef, F., Predki, P., Tay, A., Lucas, S., Richardson, P., Smith, S. F., Clark, M. S., Edwards, Y. J., Doggett, N., Zharkikh, A., Tavtigian, S. V., Pruss, D., Barnstead, M., Evans, C., Baden, H., Powell, J., Glusman, G., Rowen, L., Hood, L., Tan, Y. H., Elgar, G., Hawkins, T., Venkatesh, B., Rokhsar, D., and Brenner, S. 2002. Whole-genome shotgun assembly and analysis of the genome of Fugu rubripes. Science 297: 1301-1310.

244. Jaillon, O., Aury, J. M., Brunet, F., Petit, J. L., Stange-Thomann, N., Mauceli, E., Bouneau, L., Fischer, C., Ozouf-Costaz, C., Bernot, A., Nicaud, S., Jaffe, D., Fisher, S., Lutfalla, G., Dossat, C., Segurens, B., Dasilva, C., Salanoubat, M., Levy, M., Boudet, N., Castellano, S., Anthouard, R., Jubin, C., Castelli, V., Katinka, M., Vacherie, B., Biemont, C., Skalli, Z., Cattolico, L., Poulain, J., de Berardinis, V., Cruaud, C., Duprat, S., Brottier, P., Coutanceau, J. P., Gouzy, J., Parra, G., Lardier, G., Chapple, C., McKernan, K. J., McEwan, P., Bosak, S., Kellis, M., Volff, J. N., Guigo, R., Zody, M. C., Mesirov, J., Lindblad-Toh, K., Birren, B., Nusbaum, C., Kahn, D., Robinson-Rechavi, M., Laudet, V., Schachter, V., Quetier, F., Saurin, W., Scarpelli, C., Wincker, P., Lander, E. S., Weissenbach, J., and Crollius, H. R. 2004. Genome duplication in the teleost fish Tetraodon nigroviridis reveals the early vertebrate proto-karyotype. Nature 431: 946-957.

245. Kasahara, M., Naruse, K., Sasaki, S., Nakatani, Y., Qu, W., Ahsan, B., Yamada, T., Nagayasu, Y., Doi, K., Kasai, Y., Jindo, T., Kobayashi, D., Shimada, A., Toyoda, A., Kuroki, Y., Fujiyama, A., Sasaki, T., Shimizu, A., Asakawa, S., Shimizu, N., Hashimoto, S. I., Yang, J., Lee, Y., Matsushima, K., Sugano, S., Sakaizumi, M., Narita, T., Ohishi, K., Haga, S., Ohta, F., Nomoto, H., Nogata, K., Morishita, T., Endo, T., Shin-I, T., Takeda, H., Morishita, S., and Kohara, Y. 2007. The medaka draft genome and insights into vertebrate genome evolution. Nature 447: 714-719.

246. Yue, G. H., and Wang, L. 2017. Current status of genome sequencing and its applications in aquaculture. Aquaculture 468, Part 1: 337-347.

247. Bernardi, G., Wiley, E. O., Mansour, H., Miller, M. R., Orti, G., Haussler, D., O'Brien, S. J., Ryder, O. A., and Venkatesh, B. 2012. The fishes of Genome 10K. Mar Genom 7: 3-6. 
248. Sanger, F., Nicklen, S., and Coulson, A. R. 1977. DNA sequencing with chain-terminating inhibitors. Proceedings of the National Academy of Sciences of the United States of America 74: 5463-5467.

249. Lander, E. S., Consortium, I. H. G. S., Linton, L. M., Birren, B., Nusbaum, C., Zody, M. C., Baldwin, J., Devon, K., Dewar, K., Doyle, M., FitzHugh, W., Funke, R., Gage, D., Harris, K., Heaford, A., Howland, J., Kann, L., Lehoczky, J., LeVine, R., McEwan, P., McKernan, K., Meldrim, J., Mesirov, J. P., Miranda, C., Morris, W., Naylor, J., Raymond, C., Rosetti, M., Santos, R., Sheridan, A., Sougnez, C., Stange-Thomann, N., Stojanovic, N., Subramanian, A., Wyman, D., Rogers, J., Sulston, J., Ainscough, R., Beck, S., Bentley, D., Burton, J., Clee, C., Carter, N., Coulson, A., Deadman, R., Deloukas, P., Dunham, A., Dunham, I., Durbin, R., French, L., Grafham, D., Gregory, S., Hubbard, T., Humphray, S., Hunt, A., Jones, M., Lloyd, C., McMurray, A., Matthews, L., Mercer, S., Milne, S., Mullikin, J. C., Mungall, A., Plumb, R., Ross, M., Shownkeen, R., Sims, S., Waterston, R. H., Wilson, R. K., Hillier, L. W., McPherson, J. D., Marra, M. A., Mardis, E. R., Fulton, L. A., Chinwalla, A. T., Pepin, K. H., Gish, W. R., Chissoe, S. L., Wendl, M. C., Delehaunty, K. D., Miner, T. L., Delehaunty, A., Kramer, J. B., Cook, L. L., Fulton, R. S., Johnson, D. L., Minx, P. J., Clifton, S. W., Hawkins, T., Branscomb, E., Predki, P., Richardson, P., Wenning, S., Slezak, T., Doggett, N., Cheng, J. F., Olsen, A., Lucas, S., Elkin, C., Uberbacher, E., Frazier, M., Gibbs, R. A., Muzny, D. M., Scherer, S. E., Bouck, J. B., Sodergren, E. J., Worley, K. C., Rives, C. M., Gorrell, J. H., Metzker, M. L., Naylor, S. L., Kucherlapati, R. S., Nelson, D. L., Weinstock, G. M., Sakaki, Y., Fujiyama, A., Hattori, M., Yada, T., Toyoda, A., Itoh, T., Kawagoe, C., Watanabe, H., Totoki, Y., Taylor, T., Weissenbach, J., Heilig, R., Saurin, W., Artiguenave, F., Brottier, P., Bruls, T., Pelletier, E., Robert, C., Wincker, P., Rosenthal, A., Platzer, M., Nyakatura, G., Taudien, S., Rump, A., Yang, H. M., Yu, J., Wang, J., Huang, G. Y., Gu, J., Hood, L., Rowen, L., Madan, A., Qin, S. Z., Davis, R. W., Federspiel, N. A., Abola, A. P., Proctor, M. J., Myers, R. M., Schmutz, J., Dickson, M., Grimwood, J., Cox, D. R., Olson, M. V., Kaul, R., Raymond, C., Shimizu, N., Kawasaki, K., Minoshima, S., Evans, G. A., Athanasiou, M., Schultz, R., Roe, B. A., Chen, F., Pan, H. Q., Ramser, J., Lehrach, H., Reinhardt, R., McCombie, W. R., de la Bastide, M., Dedhia, N., Blocker, H., Hornischer, K., Nordsiek, G., Agarwala, R., Aravind, L., Bailey, J. A., Bateman, A., Batzoglou, S., Birney, E., Bork, P., Brown, D. G., Burge, C. B., Cerutti, L., Chen, H. C., Church, D., Clamp, M., Copley, R. R., Doerks, T., Eddy, S. R., Eichler, E. E., Furey, T. S., Galagan, J., Gilbert, J. G. R., Harmon, C., Hayashizaki, Y., Haussler, D., Hermjakob, H., Hokamp, K., Jang, W. H., Johnson, L. S., Jones, T. A., Kasif, S., Kaspryzk, A., Kennedy, S., Kent, W. J., Kitts, P., Koonin, E. V., Korf, I., Kulp, D., Lancet, D., Lowe, T. M., McLysaght, A., Mikkelsen, T., Moran, J. V., Mulder, N., Pollara, V. J., Ponting, C. P., Schuler, G., Schultz, J. R., Slater, G., Smit, A. F. A., Stupka, E., Szustakowki, J., Thierry-Mieg, D., Thierry-Mieg, J., Wagner, L., Wallis, J., Wheeler, R., Williams, A., Wolf, Y. I., Wolfe, K. H., Yang, S. P., Yeh, R. F., Collins, F., Guyer, M. S., Peterson, J., Felsenfeld, A., Wetterstrand, K. A., Patrinos, A., Morgan, M. J., and Conso, I. H. G. S. 2001. Initial sequencing and analysis of the human genome. Nature 409: 860-921.

250. Venter, J. C., Adams, M. D., Myers, E. W., Li, P. W., Mural, R. J., Sutton, G. G., Smith, H. O., Yandell, M., Evans, C. A., Holt, R. A., Gocayne, J. D., Amanatides, P., Ballew, R. M., Huson, D. H., Wortman, J. R., Zhang, Q., Kodira, C. D., Zheng, X. Q. H., Chen, L., Skupski, M., Subramanian, G., Thomas, P. D., Zhang, J. H., Miklos, G. L. G., Nelson, C., Broder, S., Clark, A. G., Nadeau, C., McKusick, V. A., Zinder, N., Levine, A. J., Roberts, R. J., Simon, M., Slayman, C., Hunkapiller, M., Bolanos, R., Delcher, A., Dew, I., Fasulo, D., Flanigan, M., Florea, L., Halpern, A., Hannenhalli, S., Kravitz, S., Levy, S., Mobarry, C., Reinert, K., Remington, K., Abu-Threideh, J., Beasley, E., Biddick, K., Bonazzi, V., Brandon, R., Cargill, M., Chandramouliswaran, I., Charlab, R., Chaturvedi, K., Deng, Z. M., Di Francesco, V., Dunn, P., Eilbeck, K., Evangelista, C., Gabrielian, A. E., Gan, W., Ge, W. M., Gong, F. C., Gu, Z. P., Guan, P., Heiman, T. J., Higgins, M. E., Ji, R. R., Ke, Z. X., Ketchum, K. A., Lai, Z. W., Lei, Y. D., Li, Z. Y., Li, J. Y., Liang, Y., Lin, X. Y., Lu, F., Merkulov, G. V., Milshina, N., Moore, H. M., Naik, A. K., Narayan, V. A., Neelam, B., Nusskern, D., Rusch, D. B., Salzberg, S., Shao, W., Shue, B. X., Sun, J. T., Wang, Z. Y., Wang, A. H., Wang, X., Wang, J., Wei, M. H., Wides, R., Xiao, C. L., Yan, C. H., Yao, A., Ye, J., Zhan, M., Zhang, W. Q., Zhang, H. Y., Zhao, Q., Zheng, L. S., Zhong, F., Zhong, W. Y., Zhu, S. P. C., Zhao, S. Y., Gilbert, D., Baumhueter, S., Spier, G., Carter, C., Cravchik, A., Woodage, T., Ali, F., An, H. J., Awe, A., Baldwin, D., Baden, H., Barnstead, M., Barrow, I., Beeson, K., Busam, D., Carver, A., Center, A., Cheng, M. L., Curry, L., Danaher, S., Davenport, L., Desilets, R., Dietz, S., Dodson, K., Doup, L., Ferriera, S., Garg, N., Gluecksmann, A., Hart, B., Haynes, J., Haynes, C., Heiner, C., Hladun, S., Hostin, D., Houck, J., Howland, T., Ibegwam, C., Johnson, J., Kalush, F., Kline, L., Koduru, S., Love, A., Mann, F., May, D., McCawley, S., McIntosh, T., McMullen, I., Moy, M., Moy, L., Murphy, B., Nelson, K., Pfannkoch, C., Pratts, E., Puri, V., Qureshi, H., Reardon, M., Rodriguez, R., Rogers, Y. H., Romblad, D., Ruhfel, B., Scott, R., Sitter, C., 
Smallwood, M., Stewart, E., Strong, R., Suh, E., Thomas, R., Tint, N. N., Tse, S., Vech, C., Wang, G., Wetter, J., Williams, S., Williams, M., Windsor, S., Winn-Deen, E., Wolfe, K., Zaveri, J., Zaveri, K., Abril, J. F., Guigo, R., Campbell, M. J., Sjolander, K. V., Karlak, B., Kejariwal, A., Mi, H. Y., Lazareva, B., Hatton, T., Narechania, A., Diemer, K., Muruganujan, A., Guo, N., Sato, S., Bafna, V., Istrail, S., Lippert, R., Schwartz, R., Walenz, B., Yooseph, S., Allen, D., Basu, A., Baxendale, J., Blick, L., Caminha, M., Carnes-Stine, J., Caulk, P., Chiang, Y. H., Coyne, M., Dahlke, C., Mays, A. D., Dombroski, M., Donnelly, M., Ely, D., Esparham, S., Fosler, C., Gire, H., Glanowski, S., Glasser, K., Glodek, A., Gorokhov, M., Graham, K., Gropman, B., Harris, M., Heil, J., Henderson, S., Hoover, J., Jennings, D., Jordan, C., Jordan, J., Kasha, J., Kagan, L., Kraft, C., Levitsky, A., Lewis, M., Liu, X. J., Lopez, J., Ma, D., Majoros, W., McDaniel, J., Murphy, S., Newman, M., Nguyen, T., Nguyen, N., Nodell, M., Pan, S., Peck, J., Peterson, M., Rowe, W., Sanders, R., Scott, J., Simpson, M., Smith, T., Sprague, A., Stockwell, T., Turner, R., Venter, E., Wang, M., Wen, M. Y., Wu, D., Wu, M., Xia, A., Zandieh, A., and Zhu, X. H. 2001. The sequence of the human genome. Science 291: 1304-+.

251. Margulies, M., Egholm, M., Altman, W. E., Attiya, S., Bader, J. S., Bemben, L. A., Berka, J., Braverman, M. S., Chen, Y. J., Chen, Z., Dewell, S. B., Du, L., Fierro, J. M., Gomes, X. V., Godwin, B. C., He, W., Helgesen, S., Ho, C. H., Irzyk, G. P., Jando, S. C., Alenquer, M. L., Jarvie, T. P., Jirage, K. B., Kim, J. B., Knight, J. R., Lanza, J. R., Leamon, J. H., Lefkowitz, S. M., Lei, M., Li, J., Lohman, K. L., Lu, H., Makhijani, V. B., McDade, K. E., McKenna, M. P., Myers, E. W., Nickerson, E., Nobile, J. R., Plant, R., Puc, B. P., Ronan, M. T., Roth, G. T., Sarkis, G. J., Simons, J. F., Simpson, J. W., Srinivasan, M., Tartaro, K. R., Tomasz, A., Vogt, K. A., Volkmer, G. A., Wang, S. H., Wang, Y., Weiner, M. P., Yu, P., Begley, R. F., and Rothberg, J. M. 2005. Genome sequencing in microfabricated high-density picolitre reactors. Nature 437: 376-380.

252. Zhang, Y., Stupka, E., Henkel, C. V., Jansen, H. J., Spaink, H. P., and Verbeek, F. J. 2011. Identification of common carp innate immune genes with whole-genome sequencing and RNA-Seq data. J Integr Bioinform 8: 169.

253. Henkel, C. V., Dirks, R. P., Jansen, H. J., Forlenza, M., Wiegertjes, G. F., Howe, K., van den Thillan, G. E. E. J. M., and Spaink, H. P. 2012. Comparison of the Exomes of Common Carp (Cyprinus carpio) and Zebrafish (Danio rerio). Zebrafish 9: 59-67.

254. Komen, H., and Thorgaard, G. H. 2007. Androgenesis, gynogenesis and the production of clones in fishes: A review. Aquaculture 269: 150-173.

255. Kolder, I. C., van der Plas-Duivesteijn, S. J., Tan, G., Wiegertjes, G. F., Forlenza, M., Guler, A. T., Travin, D. Y., Nakao, M., Moritomo, T., Irnazarow, I., den Dunnen, J. T., Anvar, S. Y., Jansen, H. J., Dirks, R. P., Palmblad, M., Lenhard, B., Henkel, C. V., and Spaink, H. P. 2016. A full-body transcriptome and proteome resource for the European common carp. BMC genomics 17: 701.

256. Xu, P., Zhang, X., Wang, X., Li, J., Liu, G., Kuang, Y., Xu, J., Zheng, X., Ren, L., Wang, G., Zhang, Y., Huo, L., Zhao, Z., Cao, D., Lu, C., Li, C., Zhou, Y., Liu, Z., Fan, Z., Shan, G., Li, X., Wu, S., Song, L., Hou, G., Jiang, Y., Jeney, Z., Yu, D., Wang, L., Shao, C., Song, L., Sun, J., Ji, P., Wang, J., Li, Q., Xu, L., Sun, F., Feng, J., Wang, C., Wang, S., Wang, B., Li, Y., Zhu, Y., Xue, W., Zhao, L., Wang, J., Gu, Y., Lv, W., Wu, K., Xiao, J., Wu, J., Zhang, Z., Yu, J., and Sun, X. 2014. Genome sequence and genetic diversity of the common carp, Cyprinus carpio. Nat Genet 46: 1212-1219.

257. Kohlmann, K., Gross, R., Murakaeva, A., and Kersten, P. 2003. Genetic variability and structure of common carp (Cyprinus carpio) populations throughout the distribution range inferred from allozyme, microsatellite and mitochondrial DNA markers. Aquat Living Resour 16: 421-431.

258. Xia, J. H., Liu, F., Zhu, Z. Y., Fu, J. J., Feng, J. B., Li, J. L., and Yue, G. H. 2010. A consensus linkage map of the grass carp (Ctenopharyngodon idella) based on microsatellites and SNPs. BMC genomics 11 .

259. Yang, J. X., Chen, X. L., Bai, J., Fang, D. M., Qiu, Y., Jiang, W. S., Yuan, H., Bian, C., Lu, J., He, S. Y., Pan, X. F., Zhang, Y. L., Wang, X. A., You, X. X., Wang, Y. S., Sun, Y., Mao, D. Q., Liu, Y., Fan, G. Y., Zhang, H., Chen, X. Y., Zhang, X. H., Zheng, L. P., Wang, J. T., Cheng, L., Chen, J. M., Ruan, Z. Q., Li, J., Yu, H., Peng, C., Ma, X. Y., Xu, J. M., He, Y., Xu, Z. F., Xu, P., Wang, J., Yang, H. M., Wang, J., Whitten, T., Xu, X., and Shi, Q. 2016. The Sinocyclocheilus cavefish genome provides insights into cave adaptation. BMC Biol 14 .

260. Xu, J., Li, J. T., Jiang, Y., Peng, W., Yao, Z., Chen, B., Jiang, L., Feng, J., Ji, P., Liu, G., Liu, Z., Tai, R., Dong, C., Sun, X., Zhao, Z. X., Zhang, Y., Wang, J., Li, S., Zhao, Y., Yang, J., Sun, X., and Xu, P. 2017. Genomic basis of adaptive evolution: The survival of Amur ide (Leuciscus waleckii) in an extremely alkaline environment. Mol Biol Evol 34: 145-159. 
261. Fijan, N., Sulimanovic, D., Bearzotti, M., Muzinic, D., Zwillenberg, L. O., Chilmonczyk, S., Vautherot, J. F., and Dekinkelin, P. 1983. Some properties of the Epithelioma Papulosum Cyprini (EPC) cell line from carp Cyprinus carpio. Ann Inst Pasteur Vir 134: 207-220.

262. Winton, J., Batts, W., deKinkelin, P., LeBerre, M., Bremont, M., and Fijan, N. 2010. Current lineages of the Epithelioma Papulosum Cyprini (EPC) cell line are contaminated with fathead minnow, Pimephales promelas, cells. J Fish Dis 33: 701-704.

263. Burns, F. R., Cogburn, A. L., Ankley, G. T., Villeneuve, D. L., Waits, E., Chang, Y. J., Llaca, V., Deschamps, S. D., Jackson, R. E., and Hoke, R. A. 2016. Sequencing and de novo draft assemblies of a fathead minnow (Pimephales promelas) reference genome. Environ Toxicol Chem 35: 212-217.

264. Howe, K., Clark, M. D., Torroja, C. F., Torrance, J., Berthelot, C., Muffato, M., Collins, J. E., Humphray, S., McLaren, K., Matthews, L., McLaren, S., Sealy, I., Caccamo, M., Churcher, C., Scott, C., Barrett, J. C., Koch, R., Rauch, G. J., White, S., Chow, W., Kilian, B., Quintais, L. T., Guerra-Assuncao, J. A., Zhou, Y., Gu, Y., Yen, J., Vogel, J. H., Eyre, T., Redmond, S., Banerjee, R., Chi, J., Fu, B., Langley, E., Maguire, S. F., Laird, G. K., Lloyd, D., Kenyon, E., Donaldson, S., Sehra, H., Almeida-King, J., Loveland, J., Trevanion, S., Jones, M., Quail, M., Willey, D., Hunt, A., Burton, J., Sims, S., McLay, K., Plumb, B., Davis, J., Clee, C., Oliver, K., Clark, R., Riddle, C., Elliot, D., Threadgold, G., Harden, G., Ware, D., Begum, S., Mortimore, B., Kerry, G., Heath, P., Phillimore, B., Tracey, A., Corby, N., Dunn, M., Johnson, C., Wood, J., Clark, S., Pelan, S., Griffiths, G., Smith, M., Glithero, R., Howden, P., Barker, N., Lloyd, C., Stevens, C., Harley, J., Holt, K., Panagiotidis, G., Lovell, J., Beasley, H., Henderson, C., Gordon, D., Auger, K., Wright, D., Collins, J., Raisen, C., Dyer, L., Leung, K., Robertson, L., Ambridge, K., Leongamornlert, D., McGuire, S., Gilderthorp, R., Griffiths, C., Manthravadi, D., Nichol, S., Barker, G., Whitehead, S., Kay, M., Brown, J., Murnane, C., Gray, E., Humphries, M., Sycamore, N., Barker, D., Saunders, D., Wallis, J., Babbage, A., Hammond, S., Mashreghi-Mohammadi, M., Barr, L., Martin, S., Wray, P., Ellington, A., Matthews, N., Ellwood, M., Woodmansey, R., Clark, G., Cooper, J., Tromans, A., Grafham, D., Skuce, C., Pandian, R., Andrews, R., Harrison, E., Kimberley, A., Garnett, J., Fosker, N., Hall, R., Garner, P., Kelly, D., Bird, C., Palmer, S., Gehring, I., Berger, A., Dooley, C. M., Ersan-Urun, Z., Eser, C., Geiger, H., Geisler, M., Karotki, L., Kirn, A., Konantz, J., Konantz, M., Oberlander, M., Rudolph-Geiger, S., Teucke, M., Lanz, C., Raddatz, G., Osoegawa, K., Zhu, B., Rapp, A., Widaa, S., Langford, C., Yang, F., Schuster, S. C., Carter, N. P., Harrow, J., Ning, Z., Herrero, J., Searle, S. M., Enright, A., Geisler, R., Plasterk, R. H., Lee, C., Westerfield, M., de Jong, P. J., Zon, L. I., Postlethwait, J. H., Nusslein-Volhard, C., Hubbard, T. J., Roest Crollius, H., Rogers, J., and Stemple, D. L. 2013. The zebrafish reference genome sequence and its relationship to the human genome. Nature 496: 498-503.

265. Wang, Y. P., Lu, Y., Zhang, Y., Ning, Z. M., Li, Y., Zhao, Q., Lu, H. Y., Huang, R., Xia, X. Q., Feng, Q., Liang, X. F., Liu, K. Y., Zhang, L., Lu, T. T., Huang, T., Fan, D. L., Weng, Q. J., Zhu, C. R., Lu, Y. Q., Li, W. J., Wen, Z. R., Zhou, C. C., Tian, Q. L., Kang, X. J., Shi, M. J., Zhang, W. T., Jang, S. H., Du, F. K., He, S., Liao, L. J., Li, Y. M., Gui, B., He, H. H., Ning, Z., Yang, C., He, L. B., Luo, L. F., Yang, R., Luo, Q., Liu, X. C., Li, S. S., Huang, W., Xiao, L., Lin, H. R., Han, B., and Zhu, Z. Y. 2015. The draft genome of the grass carp (Ctenopharyngodon idellus) provides insights into its evolution and vegetarian adaptation. Nat Genet 47: 625-631.

266. Dheilly, N. M., Adema, C., Raftos, D. A., Gourbal, B., Grunau, C., and Du Pasquier, L. 2014. No more non-model species: The promise of next generation sequencing for comparative immunology. Dev Comp Immunol 45: 56-66.

267. Robinson, N., Baranski, M., Das Mahapatra, K., Saha, J. N., Das, S., Mishra, J., Das, P., Kent, M., Arnyasi, M., and Sahoo, P. K. 2014. A linkage map of transcribed single nucleotide polymorphisms in rohu (Labeo rohita) and QTL associated with resistance to Aeromonas hydrophila. BMC genomics 15.

268. Kuang, Y. Y., Zheng, X. H., Li, C. Y., Li, X. M., Cao, D. C., Tong, G. X., Lv, W. H., Xu, W., Zhou, Y., Zhang, X. F., Sun, Z. P., Mahboob, S., Al-Ghanim, K. A., Li, J. T., and Sun, X. W. 2016. The genetic map of goldfish (Carassius auratus) provided insights to the divergent genome evolutions in the Cyprinidae family. Scientific reports 6: 34849.

269. Rylkova, K., Kalous, L., Slechtova, V., and Bohlen, J. 2010. Many branches, one root: First evidence for a monophyly of the morphologically highly diverse goldfish (Carassius auratus). Aquaculture 302: 36-41.

270. Pietretti, D., Spaink, H. P., Falco, A., Forlenza, M., and Wiegertjes, G. F. 2013. Accessory molecules for Toll-like receptors in Teleost fish. Identification of TLR4 interactor with leucinerich repeats (TRIL). Molecular immunology 56: 745-756.

271. Liao, X., Cheng, L., Xu, P., Lu, G., Wachholtz, M., Sun, X., and Chen, S. 2013. Transcriptome analysis of crucian carp (Carassius auratus), an important aquaculture and hypoxia-tolerant species. Plos One 8: e62308. 
272. Grabherr, M. G., Haas, B. J., Yassour, M., Levin, J. Z., Thompson, D. A., Amit, I., Adiconis, X., Fan, L., Raychowdhury, R., Zeng, Q. D., Chen, Z. H., Mauceli, E., Hacohen, N., Gnirke, A., Rhind, N., di Palma, F., Birren, B. W., Nusbaum, C., Lindblad-Toh, K., Friedman, N., and Regev, A. 2011. Full-length transcriptome assembly from RNA-Seq data without a reference genome. Nat Biotechnol 29: 644-U130.

273. Rhee, J. S., Jeong, C. B., Kim, D. H., Kim, I. C., Lee, Y. S., Lee, C., and Lee, J. S. 2014. Immune gene discovery in the crucian carp Carassius auratus. Fish \& shellfish immunology 36: $240-251$.

274. Ji, P., Liu, G., Xu, J., Wang, X., Li, J., Zhao, Z., Zhang, X., Zhang, Y., Xu, P., and Sun, X. 2012. Characterization of common carp transcriptome: sequencing, de novo assembly, annotation and comparative genomics. Plos One 7: e35152.

275. Fu, B. D., and He, S. P. 2012. Transcriptome analysis of silver carp (Hypophthalmichthys molitrix) by paired-end RNA sequencing. DNA Res 19: 131-142.

276. Gao, Z. X., Luo, W., Liu, H., Zeng, C., Liu, X. L., Yi, S. J., and Wang, W. M. 2012. Transcriptome analysis and SSR/SNP markers information of the blunt snout bream (Megalobrama amblycephala). Plos One 7.

277. Luo, H., Xiao, S. J., Ye, H., Zhang, Z. S., Lv, C. H., Zheng, S. M., Wang, Z. Y., and Wang, X. Q. 2016. Identification of immune-related genes and development of SSR/SNP markers from the spleen transcriptome of Schizothorax prenanti. Plos One 11.

278. Zhang, R., Ludwig, A., Zhang, C., Tong, C., Li, G., Tang, Y., Peng, Z., and Zhao, K. 2015. Local adaptation of Gymnocypris przewalskii (Cyprinidae) on the Tibetan Plateau. Scientific reports 5: 9780.

279. Tong, C., Zhang, C. F., Zhang, R. Y., and Zhao, K. 2015. Transcriptome profiling analysis of naked carp (Gymnocypris przewalskii) provides insights into the immune-related genes in highland fish. Fish \& shellfish immunology 46: 366-377.

280. Tong, C., Lin, Y. Q., Zhang, C. F., Shi, J. Q., Qi, H. F., and Zhao, K. 2015. Transcriptomewide identification, molecular evolution and expression analysis of Toll-like receptor family in a Tibet fish, Gymnocypris przewalskii. Fish \& shellfish immunology 46: 334-345.

281. Miyazawa, R., Matsuura, Y., Shibasaki, Y., Imamura, S., and Nakanishi, T. 2016. Crossreactivity of monoclonal antibodies against CD4-1 and CD8alpha of ginbuna crucian carp with lymphocytes of zebrafish and other cyprinid species. Dev Comp Immunol.

282. Jiang, Y., Feng, S., Zhang, S., Liu, H., Feng, J., Mu, X., Sun, X., and Xu, P. 2016. Transcriptome signatures in common carp spleen in response to Aeromonas hydrophila infection. Fish \& shellfish immunology 57: 41-48.

283. Xu, X., Shen, Y., Fu, J., Lu, L., and Li, J. 2014. De novo assembly of the grass carp Ctenopharyngodon idella transcriptome to identify miRNA targets associated with motile aeromonad septicemia. Plos One 9: e112722.

284. Xu, X. Y., Shen, Y. B., Fu, J. J., Lu, L. Q., and Li, J. L. 2015. Next-generation sequencing identified microRNAs that associate with motile aeromonad septicemia in grass carp. Fish \& shellfish immunology 45: 94-103.

285. Yang, Y., Yu, H., Li, H., and Wang, A. L. 2016. Transcriptome profiling of grass carp (Ctenopharyngodon idellus) infected with Aeromonas hydrophila. Fish \& shellfish immunology 51: 329-336.

286. Song, X., Hu, X., Sun, B., Bo, Y., Wu, K., Xiao, L., and Gong, C. 2017. A transcriptome analysis focusing on inflammation-related genes of grass carp intestines following infection with Aeromonas hydrophila. Scientific reports 7: 40777.

287. Yang, Y., Yu, H., Li, H., Wang, A., and Yu, H. Y. 2016. Effect of high temperature on immune response of grass carp (Ctenopharyngodon idellus) by transcriptome analysis. Fish \& shellfish immunology 58: 89-95.

288. Dang, Y. F., Xu, X. Y., Shen, Y. B., Hu, M. Y., Zhang, M., Li, L. S., Lv, L. Q., and Li, J. L. 2016. Transcriptome analysis of the innate immunity-related complement system in spleen tissue of Ctenopharyngodon idella infected with Aeromonas hydrophila. Plos One 11.

289. Robinson, N., Sahoo, P. K., Baranski, M., Das Mahapatra, K., Saha, J. N., Das, S., Mishra, Y., Das, P., Barman, H. K., and Eknath, A. E. 2012. Expressed sequences and polymorphisms in rohu carp (Labeo rohita, Hamilton) revealed by mRNA-seq. Marine biotechnology 14: 620633.

290. Das, S., Chhottaray, C., Das Mahapatra, K., Saha, J. N., Baranski, M., Robinson, N., and Sahoo, P. K. 2014. Analysis of immune-related ESTs and differential expression analysis of few important genes in lines of rohu (Labeo rohita) selected for resistance and susceptibility to Aeromonas hydrophila infection. Mol Biol Rep 41: 7361-7371.

291. Tran, N. T., Gao, Z. X., Zhao, H. H., Yi, S. K., Chen, B. X., Zhao, Y. H., Lin, L., Liu, X. Q., and Wang, W. M. 2015. Transcriptome analysis and microsatellite discovery in the blunt snout bream (Megalobrama amblycephala) after challenge with Aeromonas hydrophila. Fish \& shellfish immunology 45: 72-82. 
292. Cui, L., Hu, H., Wei, W., Wang, W., and Liu, H. 2016. Identification and characterization of microRNAs in the liver of blunt snout bream (Megalobrama amblycephala) infected by Aeromonas hydrophila. Int J Mol Sci 17.

293. Ramasamy, A., Mondry, A., Holmes, C. C., and Altman, D. G. 2008. Key issues in conducting a meta-analysis of gene expression microarray datasets. Plos Med 5: 1320-1332.

294. Edgar, R., Domrachev, M., and Lash, A. E. 2002. Gene expression omnibus: NCBI gene expression and hybridization array data repository. Nucleic acids research 30: 207-210.

295. Kolesnikov, N., Hastings, E., Keays, M., Melnichuk, O., Tang, Y. A., Williams, E., Dylag, M., Kurbatova, N., Brandizi, M., Burdett, T., Megy, K., Pilicheva, E., Rustici, G., Tikhonov, A., Parkinson, H., Petryszak, R., Sarkans, U., and Brazma, A. 2015. ArrayExpress updatesimplifying data submissions. Nucleic acids research 43: D1113-D1116.

296. Li, G., Zhao, Y., Wang, J., Liu, B., Sun, X., Guo, S., and Feng, J. 2016. Transcriptome profiling of developing spleen tissue and discovery of immune-related genes in grass carp (Ctenopharyngodon idella). Fish \& shellfish immunology 60: 400-410.

297. Pasquier, J., Cabau, C., Nguyen, T., Jouanno, E., Severac, D., Braasch, I., Journot, L., Pontarotti, P., Klopp, C., Postlethwait, J. H., Guiguen, Y., and Bobe, J. 2016. Gene evolution and gene expression after whole genome duplication in fish: the PhyloFish database. BMC genomics 17: 368.

298. Lee, X. Z., Yi, Y., Weng, S. P., Zeng, J., Zhang, H. T., He, J. G., and Dong, C. F. 2016. Transcriptomic analysis of koi (Cyprinus carpio) spleen tissue upon cyprinid herpesvirus-3 (CyHV3) infection using next generation sequencing. Fish \& shellfish immunology 49: 213224.

299. Chen, J., Li, C., Huang, R., Du, F. K., Liao, L. J., Zhu, Z. Y., and Wang, Y. P. 2012. Transcriptome analysis of head kidney in grass carp and discovery of immune-related genes. Bmc Vet Res 8.

300. Shi, M., Huang, R., Du, F., Pei, Y., Liao, L., Zhu, Z., and Wang, Y. 2014. RNA-seq profiles from grass carp tissues after reovirus (GCRV) infection based on singular and modular enrichment analyses. Molecular immunology 61: 44-53.

301. Wan, Q. Y., and Su, J. G. 2015. Transcriptome analysis provides insights into the regulatory function of alternative splicing in antiviral immunity in grass carp (Ctenopharyngodon idella). Scientific reports 5 .

302. Du, X., Li, Y., Li, D., Lian, F., Yang, S., Wu, J., Liu, H., Bu, G., Meng, F., Cao, X., Zeng, X., Zhang, H., and Chen, Z. 2017. Transcriptome profiling of spleen provides insights into the antiviral mechanism in Schizothorax prenanti after poly (I: C) challenge. Fish \& shellfish immunology.

303. Yuan, J., Yang, Y., Nie, H., Li, L., Gu, W., Lin, L., Zou, M., Liu, X., Wang, M., and Gu, Z. 2014. Transcriptome analysis of epithelioma papulosum cyprini cells after SVCV infection. BMC genomics 15: 935.

304. Zhong, H., Li, J. L., Zhou, Y., Li, H. X., Tang, Y. K., Yu, J. H., and Yu, F. 2016. A transcriptome resource for common carp after growth hormone stimulation. Mar Genom 25: 25-27.

305. Zhou, Y., Yu, W., Zhong, H., Li, J., Li, H., He, F., Zhou, J., Tang, Y., Yu, J., and Yu, F. 2016. Transcriptome analysis reveals that insulin is an immunomodulatory hormone in common carp. Fish \& shellfish immunology 59: 213-219.

306. Li, L., Liang, X. F., He, S., Sun, J., Wen, Z. Y., He, Y. H., Cai, W. J., Wang, Y. P., and Tao, Y. X. 2015. Transcriptome analysis of grass carp (Ctenopharyngodon idella) fed with animal and plant diets. Gene 574: 371-379.

307. Tian, J. J., Lu, R. H., Ji, H., Sun, J., Li, C., Liu, P., Lei, C. X., Chen, L. Q., and Du, Z. Y. 2015. Comparative analysis of the hepatopancreas transcriptome of grass carp (Ctenopharyngodon idellus) fed with lard oil and fish oil diets. Gene 565: 192-200.

308. Wu, S., Ren, Y., Peng, C., Hao, Y., Xiong, F., Wang, G., Li, W., Zou, H., and Angert, E. R. 2015. Metatranscriptomic discovery of plant biomass-degrading capacity from grass carp intestinal microbiomes. FEMS microbiology ecology 91.

309. He, S., Liang, X. F., Li, L., Sun, J., Wen, Z. Y., Cheng, X. Y., Li, A. X., Cai, W. J., He, Y. H., Wang, Y. P., Tao, Y. X., and Yuan, X. C. 2015. Transcriptome analysis of food habit transition from carnivory to herbivory in a typical vertebrate herbivore, grass carp Ctenopharyngodon idella. BMC genomics 16: 15.

310. He, L., Pei, Y., Jiang, Y., Li, Y., Liao, L., Zhu, Z., and Wang, Y. 2015. Global gene expression patterns of grass carp following compensatory growth. BMC genomics 16: 184.

311. Brinkmann, M., Koglin, S., Eisner, B., Wiseman, S., Hecker, M., Eichbaum, K., Thalmann, B., Buchinger, S., Reifferscheid, G., and Hollert, H. 2016. Characterisation of transcriptional responses to dioxins and dioxin-like contaminants in roach (Rutilus rutilus) using whole transcriptome analysis. Sci Total Environ 541: 412-423. 
312. Sun, S., Ge, X., Zhu, J., Zhang, W., and Xuan, F. 2016. De novo assembly of the blunt snout bream (Megalobrama amblycephala) gill transcriptome to identify ammonia exposure associated microRNAs and their targets. Results Immunol 6: 21-27.

313. Leggatt, R. A., and Iwama, G. K. 2003. Occurrence of polyploidy in the fishes. Rev Fish Biol Fisher 13: 237-246.

314. Ohno, S. 1970. Evolution by gene duplication. Springer-Verlag, Berlin, New York,.

315. Dehal, P., and Boore, J. L. 2005. Two rounds of whole genome duplication in the ancestral vertebrate. PLoS biology 3: e314.

316. Amores, A., Force, A., Yan, Y. L., Joly, L., Amemiya, C., Fritz, A., Ho, R. K., Langeland, J., Prince, V., Wang, Y. L., Westerfield, M., Ekker, M., and Postlethwait, J. H. 1998. Zebrafish hox clusters and vertebrate genome evolution. Science 282: 1711-1714.

317. Meyer, A., and Malaga-Trillo, E. 1999. Vertebrate genomics: More fishy tales about Hox genes. Curr Biol 9: R210-+.

318. Taylor, J. S., Van de Peer, Y., and Meyer, A. 2001. Genome duplication, divergent resolution and speciation. Trends Genet 17: 299-301.

319. David, L., Blum, S., Feldman, M. W., Lavi, U., and Hillel, J. 2003. Recent duplication of the, common carp (Cyprinus carpio L.) genome as revealed by analyses of microsatellite loci. Mol Biol Evol 20: 1425-1434.

320. Larhammar, D., and Risinger, C. 1994. Molecular genetic aspects of tetraploidy in the common carp Cyprinus carpio. Mol Phylogenet Evol 3: 59-68.

321. Wang, J. T., Li, J. T., Zhang, X. F., and Sun, X. W. 2012. Transcriptome analysis reveals the time of the fourth round of genome duplication in common carp (Cyprinus carpio). BMC genomics 13: 96.

322. Macqueen, D. J., and Johnston, I. A. 2014. A well-constrained estimate for the timing of the salmonid whole genome duplication reveals major decoupling from species diversification. Proc Biol Sci 281: 20132881.

323. Berthelot, C., Brunet, F., Chalopin, D., Juanchich, A., Bernard, M., Noel, B., Bento, P., Da Silva, C., Labadie, K., Alberti, A., Aury, J. M., Louis, A., Dehais, P., Bardou, P., Montfort, J., Klopp, C., Cabau, C., Gaspin, C., Thorgaard, G. H., Boussaha, M., Quillet, E., Guyomard, R., Galiana, D., Bobe, J., Volff, J. N., Genet, C., Wincker, P., Jaillon, O., Crollius, H. R., and Guiguen, Y. 2014. The rainbow trout genome provides novel insights into evolution after whole-genome duplication in vertebrates. Nature communications 5.

324. Lien, S., Koop, B. F., Sandve, S. R., Miller, J. R., Kent, M. P., Nome, T., Hvidsten, T. R., Leong, J. S., Minkley, D. R., Zimin, A., Grammes, F., Grove, H., Gjuvsland, A., Walenz, B., Hermansen, R. A., von Schalburg, K., Rondeau, E. B., Di Genova, A., Samy, J. K. A., Vik, J. O., Vigeland, M. D., Caler, L., Grimholt, U., Jentoft, S., Vage, D. I., de Jong, P., Moen, T., Baranski, M., Palti, Y., Smith, D. R., Yorke, J. A., Nederbragt, A. J., Tooming-Klunderud, A., Jakobsen, K. S., Jiang, X. T., Fan, D. D., Liberles, D. A., Vidal, R., Iturra, P., Jones, S. J. M., Jonassen, I., Maass, A., Omholt, S. W., and Davidson, W. S. 2016. The Atlantic salmon genome provides insights into rediploidization. Nature 533: 200-+.

325. Kihara, H., and Ono, T. 1926. Chromosomenzahlen und systematische Gruppierung der Rumex-Arten. Z Zellforsch Mikrosk Anat 4: 475-481.

326. Sammut, B., Marcuz, A., and Du Pasquier, L. 2002. The fate of duplicated major histocompatibility complex class Ia genes in a dodecaploid amphibian, Xenopus ruwenzoriensis. Eur J Immunol 32: 1593-1604.

327. Li, X. Y., Zhang, X. J., Li, Z., Hong, W., Liu, W., Zhang, J., and Gui, J. F. 2014. Evolutionary history of two divergent Dmrt1 genes reveals two rounds of polyploidy origins in gibel carp. Mol Phylogenet Evol 78: 96-104.

328. Yuan, J. A., He, Z. Z., Yuan, X. N., Jiang, X. Y., Sun, X. W., and Zou, S. M. 2010. Speciation of polyploid Cyprinidae fish of common carp, crucian carp, and silver crucian carp derived from duplicated Hox genes. J Exp Zool Part B 314B: 445-456.

329. Chai, J., Su, Y. B., Huang, F., Liu, S. J., Tao, M., Murphy, R. W., and Luo, J. 2015. The gap in research on polyploidization between plants and vertebrates: model systems and strategic challenges. Sci Bull 60: 1471-1478.

330. Hufton, A. L., and Panopoulou, G. 2009. Polyploidy and genome restructuring: a variety of outcomes. Curr Opin Genet Dev 19: 600-606.

331. Du Pasquier, L., Miggiano, V. C., Kobel, H. R., and Fischberg, M. 1977. The genetic control of histocompatibility reactions in natural and laboratory-made polyploid individuals of the clawed toad Xenopus. Immunogenetics 5: 129-141.

332. Session, A. M., Uno, Y., Kwon, T., Chapman, J. A., Toyoda, A., Takahashi, S., Fukui, A., Hikosaka, A., Suzuki, A., Kondo, M., van Heeringen, S. J., Quigley, I., Heinz, S., Ogino, H., Ochi, H., Hellsten, U., Lyons, J. B., Simakov, O., Putnam, N., Stites, J., Kuroki, Y., Tanaka, T., Michiue, T., Watanabe, M., Bogdanovic, O., Lister, R., Georgiou, G., Paranjpe, S. S., van Kruijsbergen, I., Shu, S., Carlson, J., Kinoshita, T., Ohta, Y., Mawaribuchi, S., Jenkins, J., 
Grimwood, J., Schmutz, J., Mitros, T., Mozaffari, S. V., Suzuki, Y., Haramoto, Y., Yamamoto, T. S., Takagi, C., Heald, R., Miller, K., Haudenschild, C., Kitzman, J., Nakayama, T., Izutsu, Y., Robert, J., Fortriede, J., Burns, K., Lotay, V., Karimi, K., Yasuoka, Y., Dichmann, D. S., Flajnik, M. F., Houston, D. W., Shendure, J., DuPasquier, L., Vize, P. D., Zorn, A. M., Ito, M., Marcotte, E. M., Wallingford, J. B., Ito, Y., Asashima, M., Ueno, N., Matsuda, Y., Veenstra, G. J., Fujiyama, A., Harland, R. M., Taira, M., and Rokhsar, D. S. 2016. Genome evolution in the allotetraploid frog Xenopus laevis. Nature 538: 336-343.

333. Koonin, E. V. 2005. Orthologs, paralogs, and evolutionary genomics. Annu Rev Genet 39: 309-338.

334. Langham, R. J., Walsh, J., Dunn, M., Ko, C., Goff, S. A., and Freeling, M. 2004. Genomic duplication, fractionation and the origin of regulatory novelty. Genetics 166: 935-945.

335. Rastogi, S., and Liberles, D. A. 2005. Subfunctionalization of duplicated genes as a transition state to neofunctionalization. BMC Evol Biol 5.

336. Force, A., Lynch, M., Pickett, F. B., Amores, A., Yan, Y. L., and Postlethwait, J. 1999. Preservation of duplicate genes by complementary, degenerative mutations. Genetics 151: 1531-1545.

337. Li, J. T., Hou, G. Y., Kong, X. F., Li, C. Y., Zeng, J. M., Li, H. D., Xiao, G. B., Li, X. M., and Sun, X. W. 2015. The fate of recent duplicated genes following a fourth-round whole genome duplication in a tetraploid fish, common carp (Cyprinus carpio). Scientific reports 5: 8199.

338. Amores, A., Catchen, J., Ferrara, A., Fontenot, Q., and Postlethwait, J. H. 2011. Genome evolution and meiotic maps by massively parallel DNA sequencing: spotted gar, an outgroup for the teleost genome duplication. Genetics 188: 799-808.

339. Seeb, J. E., Carvalho, G., Hauser, L., Naish, K., Roberts, S., and Seeb, L. W. 2011. Singlenucleotide polymorphism (SNP) discovery and applications of SNP genotyping in nonmodel organisms. Mol Ecol Resour 11 Suppl 1: 1-8.

340. Valenzuela-Quinonez, F. 2016. How fisheries management can benefit from genomics? Brief Funct Genomics 15: 352-357.

341. Baird, N. A., Etter, P. D., Atwood, T. S., Currey, M. C., Shiver, A. L., Lewis, Z. A., Selker, E. U., Cresko, W. A., and Johnson, E. A. 2008. Rapid SNP discovery and genetic mapping using sequenced RAD markers. Plos One 3.

342. Elshire, R. J., Glaubitz, J. C., Sun, Q., Poland, J. A., Kawamoto, K., Buckler, E. S., and Mitchell, S. E. 2011. A robust, simple genotyping-by-sequencing (GBS) approach for high diversity species. Plos One 6.

343. Davey, J. W., and Blaxter, M. L. 2011. RADSeq: next-generation population genetics Brief Funct Genomics 10: 108-108.

344. Peterson, B. K., Weber, J. N., Kay, E. H., Fisher, H. S., and Hoekstra, H. E. 2012. Double digest RADseq: An inexpensive method for de novo SNP discovery and genotyping in model and non-model species. Plos One 7.

345. Gjedrem, T., and Robinson, N. 2014. Advances by selective breeding for aquatic species: a review. Agric Sci 5: 1152.

346. Bell, C. J., Dinwiddie, D. L., Miller, N. A., Hateley, S. L., Ganusova, E. E., Mudge, J., Langley, R. J., Zhang, L., Lee, C. C., Schilkey, F. D., Sheth, V., Woodward, J. E., Peckham, H. E., Schroth, G. P., Kim, R. W., and Kingsmore, S. F. 2011. Carrier testing for severe childhood recessive diseases by next-generation sequencing. Sci Transl Med 3: 65ra64.

347. Kai, W., Nomura, K., Fujiwara, A., Nakamura, Y., Yasuike, M., Ojima, N., Masaoka, T., Ozaki, A., Kazeto, Y., Gen, K., Nagao, J., Tanaka, H., Kobayashi, T., and Ototake, M. 2014. A ddRAD-based genetic map and its integration with the genome assembly of Japanese eel (Anguilla japonica) provides insights into genome evolution after the teleost-specific genome duplication. BMC genomics 15.

348. Tsai, H. Y., Robledo, D., Lowe, N. R., Bekaert, M., Taggart, J. B., Bron, J. E., and Houston, R. D. 2016. Construction and annotation of a high density SNP linkage map of the Atlantic salmon (Salmo salar) genome. G3 6: 2173-2179.

349. Vij, S., Kuhl, H., Kuznetsova, I. S., Komissarov, A., Yurchenko, A. A., Van Heusden, P., Singh, S., Thevasagayam, N. M., Prakki, S. R. S., Purushothaman, K., Saju, J. M., Jiang, J., Mbandi, S. K., Jonas, M., Tong, A. H. Y., Mwangi, S., Lau, D., Ngoh, S. Y., Liew, W. C., Shen, X. Y., Hon, L. S., Drake, J. P., Boitano, M., Hall, R., Chin, C. S., Lachumanan, R., Korlach, J., Trifonov, V., Kabilov, M., Tupikin, A., Green, D., Moxon, S., Garvin, T., Sedlazeck, F. J., Vurture, G. W., Gopalapillai, G., Katneni, V. K., Noble, T. H., Scaria, V., Sivasubbu, S., Jerry, D. R., O'Brien, S. J., Schatz, M. C., Dalmay, T., Turner, S. W., Lok, S., Christoffels, A., and Orban, L. 2016. Chromosomal-level assembly of the Asian seabass genome using long sequence reads and multi-layered scaffolding. Plos Genet 12.

350. Kongchum, P., Sandel, E., Lutzky, S., Hallerman, E. M., Hulata, G., David, L., and Palti, Y. 2011. Association between $I L-10$ a single nucleotide polymorphisms and resistance to cyprinid herpesvirus-3 infection in common carp (Cyprinus carpio). Aquaculture 315: 417-421. 
351. Piazzon, M. C., Wentzel, A. S., Wiegertjes, G. F., and Forlenza, M. 2017. Carp Il10a and Il10b exert identical biological activities in vitro, but are differentially regulated in vivo. Dev Comp Immunol 67: 350-360.

352. Savan, R., Igawa, D., and Sakai, M. 2003. Cloning, characterization and expression analysis of interleukin-10 from the common carp, Cyprinus carpio L. Eur J Biochem 270: 4647-4654.

353. Shiina, T., Hosomichi, K., Inoko, H., and Kulski, J. K. 2009. The HLA genomic loci map: expression, interaction, diversity and disease. J Hum Genet 54: 15-39.

354. Harris, T. D., Buzby, P. R., Babcock, H., Beer, E., Bowers, J., Braslavsky, I., Causey, M., Colonell, J., Dimeo, J., Efcavitch, J. W., Giladi, E., Gill, J., Healy, J., Jarosz, M., Lapen, D., Moulton, K., Quake, S. R., Steinmann, K., Thayer, E., Tyurina, A., Ward, R., Weiss, H., and Xie, Z. 2008. Single-molecule DNA sequencing of a viral genome. Science 320: 106-109.

355. Muller, E. E. L., Pinel, N., Gillece, J. D., Schupp, J. M., Price, L. B., Engelthaler, D. M., Levantesi, C., Tandoi, V., Luong, K., Baliga, N. S., Korlach, J., Keim, P. S., and Wilmes, P. 2012. Genome Sequence of "Candidatus Microthrix parvicella" Bio17-1, a long-chain-fattyacid-accumulating filamentous actinobacterium from a biological wastewater treatment plant. J Bacteriol 194: 6670-6671.

356. Chaisson, M. J. P., Huddleston, J., Dennis, M. Y., Sudmant, P. H., Malig, M., Hormozdiari, F., Antonacci, F., Surti, U., Sandstrom, R., Boitano, M., Landolin, J. M., Stamatoyannopoulos, J. A., Hunkapiller, M. W., Korlach, J., and Eichler, E. E. 2015. Resolving the complexity of the human genome using single-molecule sequencing. Nature 517: 608-U163.

357. Mostovoy, Y., Levy-Sakin, M., Lam, J., Lam, E. T., Hastie, A. R., Marks, P., Lee, J., Chu, C., Lin, C., Dzakula, Z., Cao, H., Schlebusch, S. A., Giorda, K., Schnall-Levin, M., Wall, J. D., and Kwok, P. Y. 2016. A hybrid approach for de novo human genome sequence assembly and phasing. Nature methods 13: 587-+.

358. Loman, N. J., Quick, J., and Simpson, J. T. 2015. A complete bacterial genome assembled de novo using only nanopore sequencing data. Nature methods 12: 733-U751.

359. Jansen, H. J., Liem, M., Jong-Raadsen, S. A., Dufour, S., Weltzien, F.-A., Swinkels, W., Koelewijn, A., Palstra, A. P., Pelster, B., Spaink, H. P., Van den Thillart, G. E., Dirks, R. P., and Henkel, C. V. 2017. Rapid de novo assembly of the European eel genome from nanopore sequencing reads. bioRxiv.

360. Kolodziejczyk, A. A., Kim, J. K., Svensson, V., Marioni, J. C., and Teichmann, S. A. 2015. The technology and biology of single-cell RNA sequencing. Mol Cell 58: 610-620.

361. Vieira Braga, F. A., Teichmann, S. A., and Chen, X. 2016. Genetics and immunity in the era of single-cell genomics. Hum Mol Genet 25: R141-R148.

362. Macaulay, I. C., Svensson, V., Labalette, C., Ferreira, L., Hamey, F., Voet, T., Teichmann, S. A., and Cvejic, A. 2016. Single-cell RNA-sequencing reveals a continuous spectrum of differentiation in hematopoietic cells. Cell reports 14: 966-977.

363. Cao, J. L., Navis, A., Cox, B. D., Dickson, A. L., Gemberling, M., Karra, R., Bagnat, M., and Poss, K. D. 2016. Single epicardial cell transcriptome sequencing identifies Caveolin 1 as an essential factor in zebrafish heart regeneration. Development 143: 232-243.

364. Lefkovits, I. 2013. Alacrity of cells engaged in the immune response. Scandinavian journal of immunology 77: 1-12.

365. Forlenza, M. 2009. Immune responses of carp: A molecular and cellular approach to infections. In Cell Biology and Immunology. Wageningen University, Wageningen. 212.

366. Tacchi, L., Larragoite, E. T., Munoz, P., Amemiya, C. T., and Salinas, I. 2015. African lungfish reveal the evolutionary origins of organized mucosal lymphoid tissue in vertebrates. Curr Biol 25: 2417-2424.

367. Fuglem, B., Jirillo, E., Bjerkas, I., Kiyono, H., Nochi, T., Yuki, Y., Raida, M., Fischer, U., and Koppang, E. O. 2010. Antigen-sampling cells in the salmonid intestinal epithelium. Dev Comp Immunol 34: 768-774.

368. Lokka, G., and Koppang, E. O. 2016. Antigen sampling in the fish intestine. Dev Comp Immunol 64: 138-149.

369. Dalum, A. S., Griffiths, D. J., Valen, E. C., Amthor, K. S., Austbo, L., Koppang, E. O., Press, C. M., and Kvellestad, A. 2016. Morphological and functional development of the interbranchial lymphoid tissue (ILT) in Atlantic salmon (Salmo salar L.). Fish \& shellfish immunology 58: 153-164.

370. Westermann, A. J., Gorski, S. A., and Vogel, J. 2012. Dual RNA-seq of pathogen and host. Nat Rev Microbiol 10: 618-630.

371. Sato, A., Mayer, W. E., Overath, P., and Klein, J. 2003. Genes encoding putative natural killer cell C-type lectin receptors in teleostean fishes. Proceedings of the National Academy of Sciences of the United States of America 100: 7779-7784.

372. Kikuno, R., Sato, A., Mayer, W. E., Shintani, S., Aoki, T., and Klein, J. 2004. Clustering of Ctype lectin natural killer receptor-like loci in the bony fish Oreochromis niloticus. Scandinavian journal of immunology 59: 133-142. 
373. Engstad, R. E., and Robertsen, B. 1993. Recognition of Yeast-Cell Wall Glucan by Atlantic Salmon (Salmo-Salar L) Macrophages. Dev Comp Immunol 17: 319-330.

374. Ainsworth, A. J. 1994. A beta-glucan inhibitable zymosan receptor on channel catfish neutrophils. Veterinary immunology and immunopathology 41: 141-152.

375. Esteban, M. A., Rodriguez, A., and Meseguer, J. 2004. Glucan receptor but not mannose receptor is involved in the phagocytosis of Saccharomyces cerevisiae by seabream (Sparus aurata L.) blood leucocytes. Fish \& shellfish immunology 16: 447-451.

376. Nakao, M., Miura, C., Itoh, S., Nakahara, M., Okumura, K., Mutsuro, J., and Yano, T. 2004. A complement C3 fragment equivalent to mammalian C3d from the common carp (Cyprinus carpio): generation in serum after activation of the alternative pathway and detection of its receptor on the lymphocyte surface. Fish \& shellfish immunology 16: 139-149.

377. Callol, A., Roher, N., Amaro, C., and MacKenzie, S. 2013. Characterization of PAMP/PRR interactions in European eel (Anguilla anguilla) macrophage-like primary cell cultures. Fish \& shellfish immunology 35: 1216-1223.

378. Chadzinska, M., Leon-Kloosterziel, K. M., Plytycz, B., and Lidy Verburg-van Kemenade, B. M. 2008. In vivo kinetics of cytokine expression during peritonitis in carp: evidence for innate and alternative macrophage polarization. Dev Comp Immunol 32: 509-518.

379. Li, S., Chen, X., Li, X., Geng, X., Lin, R., Li, M., and Sun, J. 2015. Molecular characterization of purinergic receptor P2X4 involved in Japanese flounder (Paralichthys olivaceus) innate immune response and its interaction with ATP release channel Pannexin1. Fish \& shellfish immunology 47: 100-109.

380. Kiron, V., Kulkarni, A., Dahle, D., Vasanth, G., Lokesh, J., and Elvebo, O. 2016. Recognition of purified beta 1,3/1,6 glucan and molecular signalling in the intestine of Atlantic salmon. Dev Comp Immunol 56: 57-66.

381. Soanes, K. H., Figuereido, K., Richards, R. C., Mattatall, N. R., and Ewart, K. V. 2004. Sequence and expression of C-type lectin receptors in Atlantic salmon (Salmo salar). Immunogenetics 56: 572-584.

382. Trapnell, C., Pachter, L., and Salzberg, S. L. 2009. TopHat: discovering splice junctions with RNA-Seq. Bioinformatics 25: 1105-1111.

383. Li, H., Handsaker, B., Wysoker, A., Fennell, T., Ruan, J., Homer, N., Marth, G., Abecasis, G., Durbin, R., and Genome Project Data Processing, S. 2009. The Sequence Alignment/Map format and SAMtools. Bioinformatics 25: 2078-2079.

384. Anders, S., Pyl, P. T., and Huber, W. 2015. HTSeq--a Python framework to work with highthroughput sequencing data. Bioinformatics 31: 166-169.

385. Love, M. I., Huber, W., and Anders, S. 2014. Moderated estimation of fold change and dispersion for RNA-seq data with DESeq2. Genome biology 15: 550.

386. Robinson, M. D., McCarthy, D. J., and Smyth, G. K. 2010. edgeR: a Bioconductor package for differential expression analysis of digital gene expression data. Bioinformatics 26: 139140 .

387. McCarthy, D. J., Chen, Y., and Smyth, G. K. 2012. Differential expression analysis of multifactor RNA-Seq experiments with respect to biological variation. Nucleic acids research 40: 4288-4297.

388. Huber, W., Carey, V. J., Gentleman, R., Anders, S., Carlson, M., Carvalho, B. S., Bravo, H. C., Davis, S., Gatto, L., Girke, T., Gottardo, R., Hahne, F., Hansen, K. D., Irizarry, R. A., Lawrence, M., Love, M. I., MacDonald, J., Obenchain, V., Oles, A. K., Pages, H., Reyes, A., Shannon, P., Smyth, G. K., Tenenbaum, D., Waldron, L., and Morgan, M. 2015. Orchestrating high-throughput genomic analysis with Bioconductor. Nature methods 12: 115-121.

389. Petit, J., David, L., Dirks, R., and Wiegertjes, G. F. 2017. Genomic and transcriptomic approaches to study immunology in cyprinids: What is next? Dev Comp Immunol 75: 48-62.

390. Eden, E., Navon, R., Steinfeld, I., Lipson, D., and Yakhini, Z. 2009. GOrilla: a tool for discovery and visualization of enriched GO terms in ranked gene lists. BMC bioinformatics 10: 48 .

391. Kanehisa, M., and Goto, S. 2000. KEGG: kyoto encyclopedia of genes and genomes. Nucleic acids research 28: 27-30.

392. Xie, C., Mao, X. Z., Huang, J. J., Ding, Y., Wu, J. M., Dong, S., Kong, L., Gao, G., Li, C. Y., and Wei, L. P. 2011. KOBAS 2.0: a web server for annotation and identification of enriched pathways and diseases. Nucleic acids research 39: W316-W322.

393. Finn, R. D., Coggill, P., Eberhardt, R. Y., Eddy, S. R., Mistry, J., Mitchell, A. L., Potter, S. C., Punta, M., Qureshi, M., Sangrador-Vegas, A., Salazar, G. A., Tate, J., and Bateman, A. 2016. The Pfam protein families database: towards a more sustainable future. Nucleic acids research 44: D279-285.

394. Chiang, H. I., Zhou, H., Raudsepp, T., Jesudhasan, P. R., and Zhu, J. J. 2007. Chicken CD69 and CD94/NKG2-like genes in a chromosomal region syntenic to mammalian natural killer gene complex. Immunogenetics 59: 603-611. 
395. Edgar, R. C. 2004. MUSCLE: multiple sequence alignment with high accuracy and high throughput. Nucleic acids research 32: 1792-1797.

396. Kumar, S., Stecher, G., Li, M., Knyaz, C., and Tamura, K. 2018. MEGA X: Molecular Evolutionary Genetics Analysis across Computing Platforms. Mol Biol Evol 35: 1547-1549.

397. Whelan, S., and Goldman, N. 2001. A general empirical model of protein evolution derived from multiple protein families using a maximum-likelihood approach. Mol Biol Evol 18: 691699.

398. Felsenstein, J. 1985. Confidence-Limits on Phylogenies - an Approach Using the Bootstrap. Evolution 39: 783-791.

399. Brown, G. D. 2006. Dectin-1: a signalling non-TLR pattern-recognition receptor. Nature reviews. Immunology $6: 33-43$.

400. Shankaran, H., Wiley, H. S., and Resat, H. 2007. Receptor downregulation and desensitization enhance the information processing ability of signalling receptors. BMC systems biology 1: 48.

401. van Kooyk, Y. 2008. C-type lectins on dendritic cells: key modulators for the induction of immune responses. Biochemical Society transactions 36: 1478-1481.

402. Marshall, A. S. J., Willment, J. A., Pyz, E., Dennehy, K. M., Reid, D. M., Dri, P., Gordon, S., Wong, S. Y. C., and Brown, G. D. 2006. Human MICL (CLEC12A) is differentially glycosylated and is down-regulated following cellular activation. Eur J Immunol 36: 2159-2169.

403. Hefter, M., Lother, J., Weiss, E., Schmitt, A. L., Fliesser, M., Einsele, H., and Loeffler, J. 2017. Human primary myeloid dendritic cells interact with the opportunistic fungal pathogen Aspergillus fumigatus via the C-type lectin receptor Dectin-1. Med Mycol 55: 573-578.

404. Palma, A. S., Liu, Y., Zhang, H. T., Zhang, Y. B., McCleary, B. V., Yu, G. L., Huang, Q. L., Guidolin, L. S., Ciocchini, A. E., Torosantucci, A., Wang, D. N., Carvalho, A. L., Fontes, C. M. G. A., Mulloy, B., Childs, R. A., Feizi, T., and Chai, W. G. 2015. Unravelling Glucan Recognition Systems by Glycome Microarrays Using the Designer Approach and Mass Spectrometry. Mol Cell Proteomics 14: 974-988.

405. Liu, Y., Palma, A. S., Ten, F. Z., and Chai, W. G. 2018. Insights Into Glucan Polysaccharide Recognition Using Glucooligosaccharide Microarrays With Oxime-Linked Neoglycolipid Probes. Method Enzymol 598: 139-167.

406. Smith, A. J., Graves, B., Child, R., Rice, P. J., Ma, Z. C., Lowman, D. W., Ensley, H. E., Ryter, K. T., Evans, J. T., and Williams, D. L. 2018. Immunoregulatory Activity of the Natural Product Laminarin Varies Widely as a Result of Its Physical Properties. Journal of immunology 200: 788-799.

407. Neumann, N. F., Barreda, D. R., and Belosevic, M. 2000. Generation and functional analysis of distinct macrophage sub-populations from goldfish (Carassius auratus L.) kidney leukocyte cultures. Fish \& shellfish immunology 10: 1-20.

408. Barreda, D. R., Hanington, P. C., Walsh, C. K., Wong, P., and Belosevic, M. 2004. Differentially expressed genes that encode potential markers of goldfish macrophage development in vitro. Dev Comp Immunol 28: 727-746.

409. Kondera, E. 2011. Haematopoiesis in the head kidney of common carp (Cyprinus carpio L.): a morphological study. Fish physiology and biochemistry 37: 355-362.

410. Mitroulis, I., Ruppova, K., Wang, B. M., Chen, L. S., Grzybek, M., Grinenko, T., Eugster, A., Troullinaki, M., Palladini, A., Kourtzelis, I., Chatzigeorgiou, A., Schlitzer, A., Beyer, M., Joosten, L. A. B., Isermann, B., Lesche, M., Petzold, A., Simons, K., Henry, I., Dahl, A., Schultze, J. L., Wielockx, B., Zamboni, N., Mirtschink, P., Coskun, U., Hajishengallis, G., Netea, M. G., and Chavakis, T. 2018. Modulation of Myelopoiesis Progenitors Is an Integral Component of Trained Immunity. Cell 172: 147-+.

411. Netea, M. G., Joosten, L. A. B., Latz, E., Mills, K. H. G., Natoli, G., Stunnenberg, H. G., O'Neill, L. A. J., and Xavier, R. J. 2016. Trained immunity: A program of innate immune memory in health and disease. Science 352.

412. Sealey, W. M., Barrows, F. T., Hang, A., Johansen, K. A., Overturf, K., LaPatra, S. E., and Hardy, R. W. 2008. Evaluation of the ability of barley genotypes containing different amounts of beta-glucan to alter growth and disease resistance of rainbow trout Oncorhynchus mykiss. Anim Feed Sci Tech 141: 115-128.

413. Beaulaurier, J., Bickford, N., Gregg, J. L., Grady, C. A., Gannam, A. L., Winton, J. R., and Hershberger, P. K. 2012. Susceptibility of Pacific Herring to Viral Hemorrhagic Septicemia Is Influenced by Diet. J Aquat Anim Health 24: 43-48.

414. Bekkering, S., Quintin, J., Joosten, L. A., van der Meer, J. W., Netea, M. G., and Riksen, N. P. 2014. Oxidized low-density lipoprotein induces long-term proinflammatory cytokine production and foam cell formation via epigenetic reprogramming of monocytes. Arteriosclerosis, thrombosis, and vascular biology 34: 1731-1738.

415. Rizzetto, L., Ifrim, D. C., Moretti, S., Tocci, N., Cheng, S. C., Quintin, J., Renga, G., Oikonomou, V., De Filippo, C., Weil, T., Blok, B. A., Lenucci, M. S., Santos, M. A., Romani, 
L., Netea, M. G., and Cavalieri, D. 2016. Fungal Chitin Induces Trained Immunity in Human Monocytes during Cross-talk of the Host with Saccharomyces cerevisiae. The Journal of biological chemistry 291: 7961-7972.

416. van Splunter, M., van Osch, T. L. J., Brugman, S., Savelkoul, H. F. J., Joosten, L. A. B., Netea, M. G., and van Neerven, R. J. J. 2018. Induction of Trained Innate Immunity in Human Monocytes by Bovine Milk and Milk-Derived Immunoglobulin G. Nutrients 10.

417. Schrum, J. E., Crabtree, J. N., Dobbs, K. R., Kiritsy, M. C., Reed, G. W., Gazzinelli, R. T., Netea, M. G., Kazura, J. W., Dent, A. E., Fitzgerald, K. A., and Golenbock, D. T. 2018. Cutting Edge: Plasmodium falciparum Induces Trained Innate Immunity. Journal of immunology 200: 1243-1248.

418. Bekkering, S., Joosten, L. A. B., van der Meer, J. W. M., Netea, M. G., and Riksen, N. P. 2015. The Epigenetic Memory of Monocytes and Macrophages as a Novel Drug Target in Atherosclerosis. Clin Ther 37: 914-923.

419. Bekkering, S., Joosten, L. A., van der Meer, J. W., Netea, M. G., and Riksen, N. P. 2013. Trained innate immunity and atherosclerosis. Current opinion in lipidology 24: 487-492.

420. Christ, A., Gunther, P., Lauterbach, M. A. R., Duewell, P., Biswas, D., Pelka, K., Scholz, C. J., Oosting, M., Haendler, K., Bassler, K., Klee, K., Schulte-Schrepping, J., Ulas, T., Moorlag, S., Kumar, V., Park, M. H., Joosten, L. A. B., Groh, L. A., Riksen, N. P., Espevik, T., Schlitzer, A., Li, Y., Fitzgerald, M. L., Netea, M. G., Schultze, J. L., and Latz, E. 2018. Western Diet Triggers NLRP3-Dependent Innate Immune Reprogramming. Cell 172: 162-175 e114.

421. Davis, K. B. 2006. Management of physiological stress in finfish aquaculture. $N$ Am J Aquacult 68: $116-121$.

422. Lochmiller, R. L., and Deerenberg, C. 2000. Trade-offs in evolutionary immunology: just what is the cost of immunity? Oikos 88: 87-98.

423. Renshaw, S. A., Loynes, C. A., Trushell, D. M., Elworthy, S., Ingham, P. W., and Whyte, M. K. 2006. A transgenic zebrafish model of neutrophilic inflammation. Blood 108: 3976-3978.

424. Ellett, F., Pase, L., Hayman, J. W., Andrianopoulos, A., and Lieschke, G. J. 2011. mpeg1 promoter transgenes direct macrophage-lineage expression in zebrafish. Blood 117: e49-56.

425. Yao, Y. S., Jeyanathan, M., Haddadi, S., Barra, N. G., Vaseghi-Shanjani, M., Damjanovic, D., Lai, R., Afkhami, S., Chen, Y. H., Dvorkin-Gheva, A., Robbins, C. S., Schertzer, J. D., and Xing, Z. 2018. Induction of Autonomous Memory Alveolar Macrophages Requires T Cell Help and Is Critical to Trained Immunity. Cell 175: 1634-+.

426. de Vrese, M., and Schrezenmeir, J. 2008. Probiotics, prebiotics, and synbiotics. Advances in biochemical engineering/biotechnology 111: 1-66.

427. Ringo, E., Olsen, R. E., Gifstad, T. O., Dalmo, R. A., Amlund, H., Hemre, G. I., and Bakke, A. M. 2010. Prebiotics in aquaculture: a review. Aquacult Nutr 16: 117-136.

428. Dimitroglou, A., Merrifield, D. L., Carnevali, O., Picchietti, S., Avella, M., Daniels, C., Guroy, D., and Davies, S. J. 2011. Microbial manipulations to improve fish health and production A Mediterranean perspective. Fish \& shellfish immunology 30: 1-16.

429. Akhter, N., Wu, B., Memon, A. M., and Mohsin, M. 2015. Probiotics and prebiotics associated with aquaculture: A review. Fish \& shellfish immunology 45: 733-741.

430. Hoseinifar, S. H., Esteban, M. A., Cuesta, A., and Sun, Y. Z. 2015. Prebiotics and Fish Immune Response: A Review of Current Knowledge and Future Perspectives. Rev Fish Sci Aquac 23: 315-328.

431. Ma, X. J., Lv, S. B., Zhang, C., and Yang, C. P. 2013. Histone deacetylases and their functions in plants. Plant Cell Rep 32: 465-478.

432. Marks, P. A., Miller, T., and Richon, V. M. 2003. Histone deacetylases. Curr Opin Pharmacol 3: 344-351.

433. Usami, M., Kishimoto, K., Ohata, A., Miyoshi, M., Aoyama, M., Fueda, Y., and Kotani, J. 2008. Butyrate and trichostatin A attenuate nuclear factor kappa B activation and tumor necrosis factor alpha secretion and increase prostaglandin E-2 secretion in human peripheral blood mononuclear cells. Nutr Res 28: 321-328.

434. Vinolo, M. A. R., Hatanaka, E., Lambertucci, R. H., Newsholme, P., and Curi, R. 2009. Effects of short chain fatty acids on effector mechanisms of neutrophils. Cell biochemistry and function 27: 48-55.

435. Ang, Z. W., Er, J. Z., Tan, N. S., Lu, J. H., Liou, Y. C., Grosse, J., and Ding, J. L. 2016. Human and mouse monocytes display distinct signalling and cytokine profiles upon stimulation with FFAR2/FFAR3 short-chain fatty acid receptor agonists. Scientific reports 6.

436. Tall, G. G., Barbieri, M. A., Stahl, P. D., and Horazdovsky, B. F. 2001. Ras-activated endocytosis is mediated by the Rab5 guanine nucleotide exchange activity of RIN1. Dev Cell 1: 73-82.

437. Wang, Y., Waldron, R. T., Dhaka, A., Patel, A., Riley, M. M., Rozengurt, E., and Colicelli, J. 2002. The RAS effector RIN1 directly competes with RAF and is regulated by 14-3-3 proteins. Mol Cell Biol 22: 916-926. 
438. Herbin, O., Regelmann, A. G., Ramkhelawon, B., Weinstein, E. G., Moore, K. J., and Alexandropoulos, K. 2016. Monocyte Adhesion and Plaque Recruitment During Atherosclerosis Development Is Regulated by the Adapter Protein Chat-H/SHEP1. Arterioscl Throm Vas 36: 1791-1801.

439. Sakakibara, A., Ohba, Y., Kurokawa, K., Matsuda, M., and Hattori, S. 2002. Novel function of Chat in controlling cell adhesion via Cas-Crk-C3G-pathway-mediated Rap1 activation. J Cell Sci 115: 4915-4924.

440. Arnold, I. C., Paul, W. D., and Genhong, C. 2005. Rip2: A Key Molecule that Regulates both Innate and Acquired Immunity. Current Medicinal Chemistry - Anti-Inflammatory \& AntiAllergy Agents 4: 35-42.

441. Cohen, L. A., Gutierrez, L., Weiss, A., Leichtmann-Bardoogo, Y., Zhang, D. L., Crooks, D. R., Sougrat, R., Morgenstern, A., Galy, B., Hentze, M. W., Lazaro, F. J., Rouault, T. A., and Meyron-Holtz, E. G. 2010. Serum ferritin is derived primarily from macrophages through a nonclassical secretory pathway. Blood 116: 1574-1584.

442. Shouval, D. S., Ouahed, J., Biswas, A., Goettel, J. A., Horwitz, B. H., Klein, C., Muise, A. M., and Snapper, S. B. 2014. Interleukin 10 receptor signaling: master regulator of intestinal mucosal homeostasis in mice and humans. Advances in immunology 122: 177-210.

443. Rand, T. G., Sun, M., Gilyan, A., Downey, J., and Miller, J. D. 2010. Dectin-1 and inflammation-associated gene transcription and expression in mouse lungs by a toxic $(1,3)$ beta-D glucan. Archives of toxicology 84: 205-220.

444. Nakagawa, T., Roth, W., Wong, P., Nelson, A., Farr, A., Deussing, J., Villadangos, J. A., Ploegh, H., Peters, C., and Rudensky, A. Y. 1998. Cathepsin L: critical role in Ii degradation and CD4 T cell selection in the thymus. Science 280: 450-453.

445. Beers, C., Honey, K., Fink, S., Forbush, K., and Rudensky, A. 2003. Differential regulation of cathepsin S and cathepsin L in interferon gamma-treated macrophages. J Exp Med 197: 169179.

446. Pickard, M. R., and Williams, G. T. 2014. BCL2L14 (BCL2-like 14 (apoptosis facilitator)). Atlas of Genetics and Cytogenetics in Oncology and Haematology.

447. Gundra, U. M., Girgis, N. M., Ruckerl, D., Jenkins, S., Ward, L. N., Kurtz, Z. D., Wiens, K. E., Tang, M. S., Basu-Roy, U., Mansukhani, A., Allen, J. E., and Loke, P. 2014. Alternatively activated macrophages derived from monocytes and tissue macrophages are phenotypically and functionally distinct. Blood 123: e110-122.

448. Ma, Y. Y., He, X. J., Wang, H. J., Xia, Y. J., Wang, S. L., Ye, Z. Y., and Tao, H. Q. 2011. Interaction of coagulation factors and tumor-associated macrophages mediates migration and invasion of gastric cancer. Cancer science 102: 336-342.

449. Swisher, J. F. A., Khatri, U., and Feldman, G. M. 2007. Annexin A2 is a soluble mediator of macrophage activation. Journal of leukocyte biology 82: 1174-1184.

450. Fitzgerald, K. A. 2011. The Interferon Inducible Gene: Viperin. J Interf Cytok Res 31: 131135.

451. Eom, J., Yoo, J., Kim, J. J., Lee, J. B., Choi, W., Park, C. G., and Seo, J. Y. 2018. Viperin Deficiency Promotes Polarization of Macrophages and Secretion of M1 and M2 Cytokines. Immune Netw 18 .

452. Mitra, N., Banda, K., Altheide, T. K., Schaffer, L., Johnson-Pais, T. L., Beuten, J., Leach, R. J., Angata, T., Varki, N., and Varki, A. 2011. SIGLEC12, a Human-specific Segregating (Pseudo)gene, Encodes a Signaling Molecule Expressed in Prostate Carcinomas. Journal of Biological Chemistry 286: 23003-23011.

453. Cang, C. L., Bekele, B., and Ren, D. J. 2014. The voltage-gated sodium channel TPC1 confers endolysosomal excitability. Nat Chem Biol 10: 463-469.

454. Vetvicka, V., and Sima, P. 2004. $\beta$-Glucan in invertebrates. Invertebrate Survival Journal: $60-65 \%$ V 61.

455. Royet, J., Gupta, D., and Dziarski, R. 2011. Peptidoglycan recognition proteins: modulators of the microbiome and inflammation. Nature reviews. Immunology 11: 837-851.

456. Dziarski, R., and Gupta, D. 2006. The peptidoglycan recognition proteins (PGRPs). Genome biology 7: 232.

457. Lee, M. H., Osaki, T., Lee, J. Y., Baek, M. J., Zhang, R., Park, J. W., Kawabata, S., Soderhall, K., and Lee, B. L. 2004. Peptidoglycan recognition proteins involved in 1,3-beta-D-glucandependent prophenoloxidase activation system of insect. The Journal of biological chemistry 279: 3218-3227.

458. Sun, L. Y., Liu, S. K., Wang, R. J., Li, C., Zhang, J. R., and Liu, Z. J. 2014. Pathogen recognition receptors in channel catfish: IV. Identification, phylogeny and expression analysis of peptidoglycan recognition proteins. Dev Comp Immunol 46: 291-299.

459. Yu, Z. L., Li, J. H., Xue, N. N., Nie, P., and Chang, M. X. 2014. Expression and functional characterization of PGRP6 splice variants in grass carp Ctenopharyngodon idella. Dev Comp Immunol 47: 264-274. 
460. Zhang, F. M., Shan, S. J., Xu, X. Y., Wang, Y., Zhang, Y. H., Yin, M., and Yang, G. W. 2019. Molecular characterization and expression analysis of two peptidoglycan recognition proteins (CCPGRP5, CCPGRP6) in larvae ontogeny of common carp Cyprinus carpio L. and upon immune stimulation by bacteria. Bmc Vet Res 15.

461. Burton, T., Lyons, H., Lerat, Y., Stanley, M., and Rasmussen, M. B. 2009. A Review of the Potential of Marine Algae as a Source of Biofuel in Ireland. Dublin: Sustainable Energy Ireland - SEI.

462. Berri, M., Olivier, M., Holbert, S., Dupont, J., Demais, H., Le Goff, M., and Collen, P. N. 2017. Ulvan from Ulva armoricana (Chlorophyta) activates the PI3K/Akt signalling pathway via TLR4 to induce intestinal cytokine production. Algal Res 28: 39-47.

463. Lahaye, M., and Robic, A. 2007. Structure and functional properties of Ulvan, a polysaccharide from green seaweeds. Biomacromolecules 8: 1765-1774.

464. Castro, R., Zarra, I., and Lamas, J. 2004. Water-soluble seaweed extracts modulate the respiratory burst activity of turbot phagocytes. Aquaculture 229: 67-78.

465. Castro, R., Piazzon, M. C., Zarra, I., Leiro, J., Noya, M., and Lamas, J. 2006. Stimulation of turbot phagocytes by Ulva rigida C. Agardh polysaccharides. Aquaculture 254: 9-20.

466. Quezada-Rodriguez, P. D., and Fajer-Avila, E. J. 2017. The dietary effect of ulvan from Ulva clathrata on hematological-immunological parameters and growth of tilapia (Oreochromis niloticus). J Appl Phycol 29: 423-431.

467. Guriec, N., Bussy, F., Gouin, C., Mathiaud, O., Quero, B., Le Goff, M., and Collen, P. N. 2018. Ulvan Activates Chicken Heterophils and Monocytes Through Toll-Like Receptor 2 and TollLike Receptor 4. Front Immunol 9.

468. Ordovas, J. M., Ferguson, L. R., Tai, E. S., and Mathers, J. C. 2018. Personalised nutrition and health. bmj 361: bmj. k2173. 


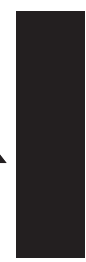




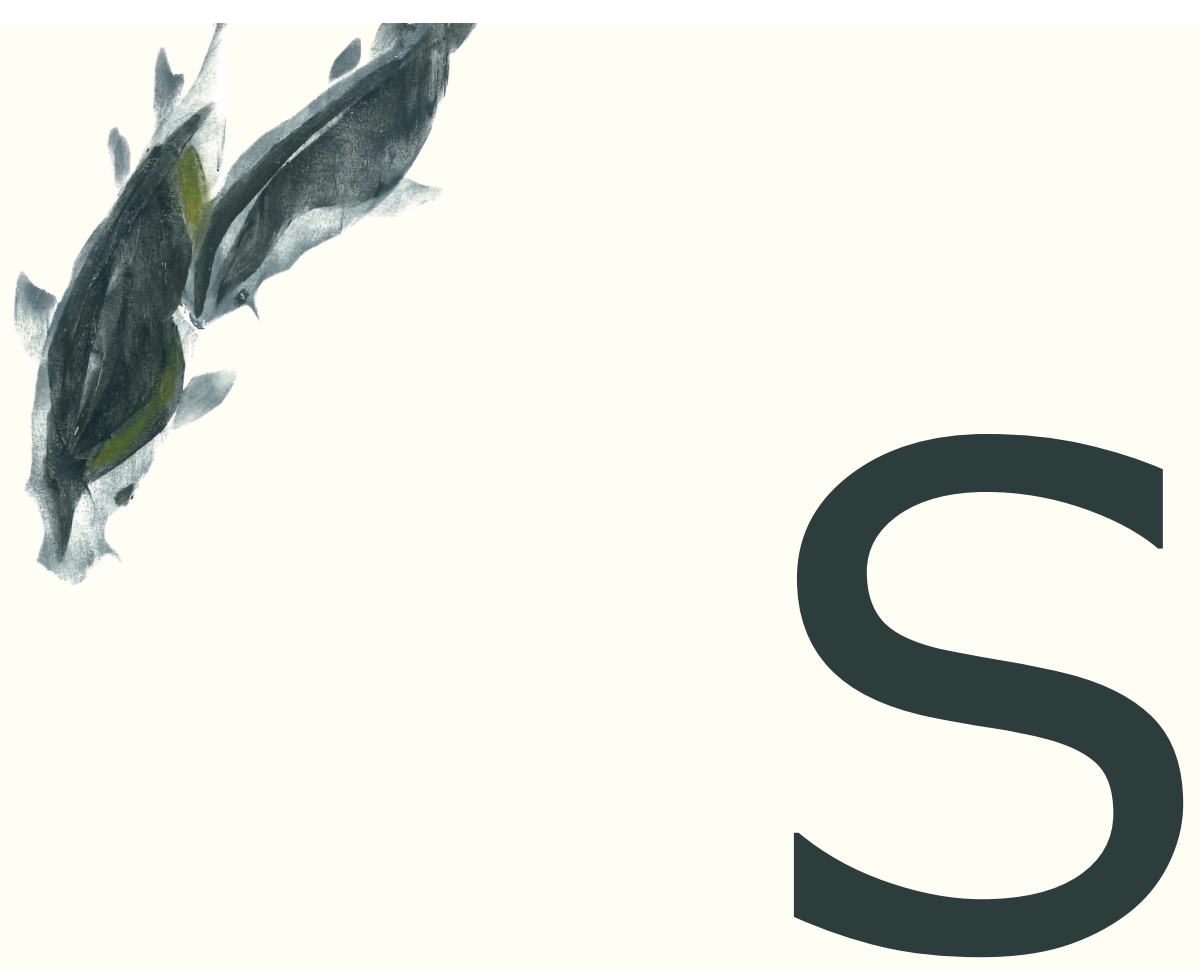

Summary (English)

Samenvatting (Nederlands)

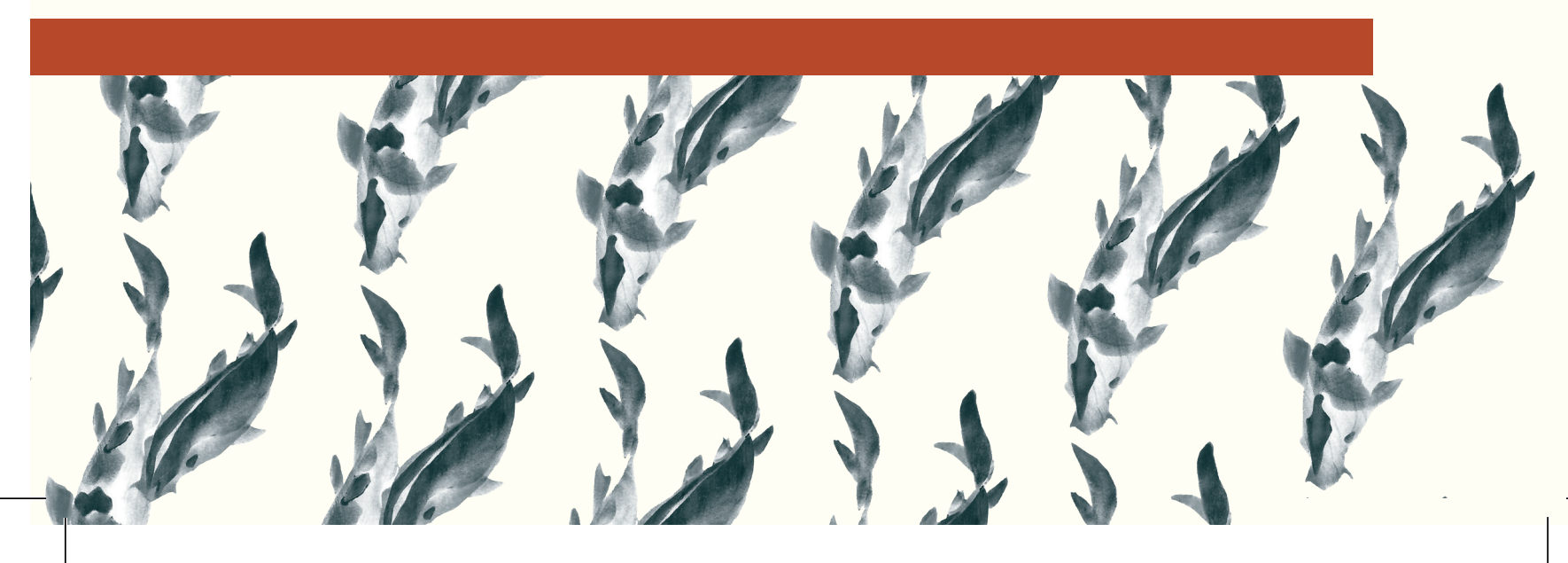




\section{Summary}

The past decades the aquaculture sector has grown tremendously to meet the growing demand for animal protein sources. The intensification of the aquaculture industry accommodated for the growth of the sector and the growing demand for fish, however increased stress, lack of adaptation to local infectious agents, increased host contact rates due to high stocking density and intensive monoculture have resulted in a clear increase in disease incidence. Overall, the immuno-modulation induced by $\beta$-glucan supplementation in fish have been well studied and are regarded as positive and beneficial for fish health. Dietary $\beta$-glucan supplementation is widely applied in aquaculture but no definitive mechanism of action has been described to date in fish. Improved understanding of the underlying mechanisms explaining the immuno-modulatory effects of $\beta$-glucans would allow for optimization of feeding and supplementation strategies.

In light of this, the main aim of this thesis was to characterize mechanisms of modulation of innate immune responses of carp by $\beta$-glucans. Using present mammalian literature as a starting point, we investigated three potential mechanisms that could be at play in the immuno-modulatory effects of $\beta$-glucan supplementation in fish, 1 ) long-lived effects on the myeloid cell compartment due to the induction of trained immunity by $\beta$-glucans; 2 ) degradation and fermentation of $\beta$-glucans by the intestinal microbiota, resulting in immunomodulation via metabolites produced by the intestinal microbiota; 3) direct receptor recognition and downstream signalling, inducing innate immune modulation.

In chapter 1, I start with delineating the rationale behind the research project and scientific collaboration between and The Netherlands Organisation for Scientific Research (NWO). Subsequently, I provide the theoretical framework for this thesis by first introducing the increasing pressure on the aquaculture industry. This increasing pressure results in intensification of the sector and increased disease incidence. Subsequently, I discuss the use of $\beta$-glucans to modulate the immune system of farmed fish. Finally I treat different mechanisms potentially explaining the effects observed in fish after supplementation with $\beta$-glucans, differentiating between direct and indirect effects.

First in chapter 2 we review the current literature on $\beta$-glucan research in fish. We sought to update previous reviews in the field, and grouped recent literature by (super)order, differentiating salmonids, perciforms and cyprinids based on the assumption that the closer the phylogenetic relationship the more reliable the conclusions. Subsequently, we review evidence in fish studies for proposed effector mechanisms involved in the immune-stimulatory effects induced by $\beta$-glucans, including intestinal microbiota, receptor recognition and downstream signalling, and a relatively new immunological concept of trained immunity. Subsequently, we revisited the screened literature for overlooked indications of long-lived effects of $\beta$-glucans in fish. We uncovered several studies with indications for long-lived 
effects in fish, however due to suboptimal experimental set-ups it was not yet possible to ascribe these observations to trained immunity.

As we observed indications for long-lived effects of $\beta$-glucans in fish in the current literature, but were made under suboptimal experimental conditions to ascribe the long-lived effects to trained immunity, we established in chapter $\mathbf{3}$ an in vitro model to investigate the conservation of trained immunity in fish. In mammals, the two main induction routes for trained immunity are via Dectin-1 or via NOD2. To date a definitive receptor and signalling pathway for $\beta$-glucans remains elusive in fish, however evidence for the conservation of NOD receptors and their downstream signalling cascade in fish is available, therefore experimental model was established with a NOD-ligand. Unstimulated trained macrophages displayed evidence of metabolic reprogramming, as well as heightened phagocytosis and increased expression of the inflammatory cytokines il6 and tnfa. Stimulated, trained macrophages showed heightened production of reactive oxygen and nitrogen species as compared to the corresponding stimulated but untrained cells. After establishment of the in vitro model, we tested laminarin, a soluble $\beta$-glucan, for its capacity to induce trained immunity in carp macrophages and observed a clear increase in ROS production in stimulated, laminarin trained macrophages compared to stimulated untrained macrophages.

After we observed conservation of trained immunity in fish and could induce trained immunity-like profiles in macrophages with $\beta$-glucans, we set out to investigate other mechanisms at play in the immuno-modulation by $\beta$-glucans. In chapter 4 we investigate whether or not the intestinal microbiota could be of importance in the effects of dietary $\beta$-glucan supplementation. First we characterised the active microbiota of naive common carp by 165 rRNA sequencing. We hypothesized based on the abundance of several genera, that the intestinal microbiota should theoretically be able to ferment $\beta$-glucans. Subsequently, an in vitro batch culture experiment was performed, confirming not only the capacity of carp intestinal microbiota to ferment $\beta$-glucans but also revealed a specific propionate production for one of the analysed $\beta$-glucans. Subsequently, a single oral gavage with this $\beta$-glucan was performed to analyse in vivo effects on the intestinal microbiota and the local gene expression. At day 7 post treatment, the $\beta$-glucan treated grouped showed a shift in active microbiota and regulation of the pro-inflammatory genes, $i l 1 \beta$, il6 and tnfa in the intestine. Taking the present literature on immune-modulatory effects of metabolites produced by the intestinal microbiota upon degradation and fermentation of dietary fibres into account, we conclude by hypothesizing that the regulation observed in the intestine after $\beta$-glucan supplementation can be a consequence of the microbiota fermenting $\beta$-glucans.

Finally, to investigate the last mechanisms proposed at the beginning of this project, we investigated possibilities of employing next generation sequencing (NGS) techniques to address this mechanisms. In chapter $\mathbf{5}$ we reviewed the current genome assemblies in teleost species and summarized current NGS-based 
studies investigating fish immune responses. We discuss the complexities and potential pitfalls of using NGS approaches with polyploid species, like common carp. Finally, we provided an outlook for coming technological advantages, which could be employed in addressing more complex issues such as genome assembly in a tetraploid species. After assessing the state of art of NGS-based studies in fish immunology, in chapter 6 we used a NGS based transcriptome analysis of macrophages stimulated with $\beta$-glucans. Using a primary culture of common carp macrophages ascertained a reduction in background due to other leukocytes. Based on differential gene expression, we could discern regulation of several signalling pathways. Furthermore, manual pathway analysis revealed the regulation of the C-type lectin receptor signalling pathway. As the regulation of this pathway suggests at least partial conservation of a $\beta$-glucan recognition cascade via a C-type lectin receptor, we investigated the current common carp genome assembly for candidate receptors. To this end, we investigated the CLR family members identified in the latest carp genome assembly and based on expression and structural architecture, we proposed several candidate receptors.

Finally, I integrate the results of the previous chapters in a larger framework and discuss the implications of these findings for future research and applications of $\beta$ glucans in aquaculture in chapter 7. I start with reflecting on the observed induction of trained immunity in carp and discuss the parallels and differences between our observations and the studies on trained immunity in mammals. Furthermore, I discuss both the potential and the possible dark-side of trained immunity for the aquaculture sector. Finally I propose two future studies which might provide useful insights from a fundamental and an applied point of view. I continue by discussing our observations on the effects of $\beta$-glucans on carp intestinal microbiota. Besides placing our observations in a broader context, I discuss the potential implications of the presence of fermentation products and $\beta$ glucans in light of trained immunity. Subsequently, I take our observations on the regulation of the C-type lectin receptor signalling pathway a step further by comparing the dataset to new datasets. By comparison to macrophages stimulated with a different $\beta$-glucan, zymosan, or with LPS, as a common pro-inflammatory agent, we can identify a specific $\beta$-glucan-regulated set of genes. I continue with the investigation of a $\beta$-glucan recognition mechanism, peptidoglycan recognition proteins (PGRPs) and the conservation of its role from invertebrates to fish. We observe indications for a role for the PGRPs in the response to $\beta$-glucans. Finally, I recognize that there are other fish in the sea of immuno-modulatory substances and discuss the use of marine sulphated polysaccharides as an alternative to $\beta$ glucans. Although during the discussion I already reflected on the relevance of our observations to the aquaculture practice, I consciously reflect on relevance of my data by addressing both its short- and long-term implications on the aquaculture sector.

The diverse angles of approach taken in the studies in this thesis have allowed a better understanding of the underlying mechanisms possibly explaining effects of $\beta$-glucan supplementation in common carp. This improved understanding of $\beta$ - 
glucan induced immuno-modulation will provide a strong base for future studies. In the long-term, the findings in this thesis might aid in achieving concepts such as "personalized feeding", similar to "personalized nutrition". Fish health could be improved through these optimized feeding strategies and reduce the need to employ other measures to improve health, such as antibiotics or vaccination strategies. 


\section{Samenvatting}

Om aan de toenemende vraag naar dierlijke eiwitbronnen te kunnen voldoen is de aquacultuur sector de laatste decennia gigantisch gegroeid. De aquacultuur sector heeft deze groei kunnen bewerkstelligen dankzij sterke intensivering. Deze intensivering heeft echter een keerzijde, namelijk een duidelijke toename aan infecties. Deze toename is onder andere het gevolg van zowel een toename aan stress en vis tot vis contact, inherent aan een hoge visdichtheid, alsmede een gebrek aan aanpassing aan de lokale ziekteverwekkers. De immuun-modulatie als gevolg van $\beta$-glucaan suppletie van visvoeders, is over het algemeen goed bestudeerd en wordt beschouwd als positief voor de gezondheid van de vissen. Ondanks de brede toepassing van $\beta$-glucaan suppletie in de aquacultuur sector zijn de definitieve mechanismen nog niet beschreven. Betere kennis van de mechanismen die aan de immuun-modulatie door $\beta$-glucaan suppletie ten grondslag liggen, zou verdere optimalisatie en verbetering van de voer- en suppletiestrategieën mogelijk kunnen maken.

Met dat in het achterhoofd was het hoofddoel van dit proefschrift het karakteriseren van de onderliggende mechanismen van de immuun-modulatie door $\beta$-glucanen in karpers. Op basis van de huidige literatuur op het gebied van immuun-modulatie door $\beta$-glucanen in zoogdieren, zijn er drie potentiele mechanismen onderzocht die een rol kunnen spelen bij de immuun-modulatie veroorzaakt door $\beta$-glucanen in vissen. De onderzochte mechanismen zijn de volgende: 1) langdurige effecten op het myeloïde cel compartiment door de inductie van 'trained immunity' door $\beta$-glucanen; 2 ) de afbraak en fermentatie van de $\beta$-glucanen door de microbiota in de darm, waarbij metabolieten geproduceerd door de microbiota indirect leiden tot immuun-modulatie; 3 ) directe activatie van een receptor door $\beta$-glucanen die via signaalcascades uiteindelijk het aangeboren immuun systeem moduleert.

In hoofdstuk 1, begin ik met het uiteenzetten van de rationaal achter het onderzoeksproject en achter de wetenschappelijke samenwerking tussen de São Paulo Research Foundation (FAPESP) en de Nederlandse Organisatie voor Wetenschappelijk Onderzoek (NWO). Vervolgens zet ik het theoretische kaderwerk voor deze thesis uiteen door de toenemende druk op de aquacultuur industrie te introduceren. Deze toenemende druk heeft ertoe geleid dat de sector sterk heeft moeten intensiveren, wat leidde tot een toename in ziekte incidentie. Vervolgens bediscussieer ik het gebruik van $\beta$-glucanen om het immuunsysteem van kweekvissen te moduleren. Tenslotte, bespreek ik verschillende mechanismen die mogelijk de geobserveerde effecten van $\beta$-glucanen in vissen zouden kunnen verklaren, hierbij differentieer ik tussen directe en indirecte effecten.

In hoofdstuk 2, behandelen we de huidige literatuur over onderzoek naar $\beta$ glucanen in vissen. We wilden eerdere reviews over dit onderwerp updaten om op die manier een concreet overzicht van de huidige literatuur op het gebied van immuun-modulatie door $\beta$-glucanen in vissen te krijgen. We hebben daartoe 
recente literatuur gegroepeerd per (super)orde, waarbij we onderscheid maken tussen zalmachtigen, baarsachtigen en karperachtigen. Dit onderscheid maken we omdat we aannemen dat nauwere fylogenetische verwantschappen tot betrouwbaardere conclusies kunnen leiden. Vervolgens analyseren we de huidige literatuur voor indicaties dat de voorgestelde effector mechanismen een rol zouden kunnen spelen in de immuun-modulatie door $\beta$-glucanen in vissen. Deze eerder genoemde mechanismen zijn de effecten via de microbiota in de darm, directe receptor herkenning leidend tot activatie van de signaalcascade en het relatief nieuwe concept trained immunity. Vervolgens, zijn we opnieuw door de literatuur gegaan op zoek naar mogelijke langdurige effecten van $\beta$-glucanen in vissen die over het hoofd gezien zijn. We vinden een aantal studies waar dergelijke langdurige effecten in vissen beschreven worden, echter doordat de experimenten niet opgezet zijn om deze langdurige effecten te onderzoeken kunnen we deze observaties niet toeschrijven aan trained immunity.

Omdat we indicaties voor langdurige effecten van $\beta$-glucanen in vis vonden in de literatuur, maar we vanwege suboptimale opzet van experimenten deze observaties niet konden toeschrijven aan trained immunity, hebben we in hoofdstuk $\mathbf{3}$ een in vitro model opgezet om de evolutionaire conservatie van trained immunity in vissen te onderzoeken. In zoogdieren zijn de twee best beschreven routes om trained immunity te induceren via Dectin-1 of via NOD2. Tot de dag van vandaag is er nog geen definitieve receptor-route of signaalcascade voor $\beta$-glucanen in vis beschreven, er is echter wel bewijs voor evolutionaire conservatie van de NOD receptoren en hun signaalcascade in vissen. Derhalve hebben we het experimentele model opgezet met een NOD-ligand. Ongestimuleerde getrainde karper macrofagen lieten naast kenmerken van metabole herprogrammering ook een toename in fagocytose activiteiten en een toename in expressie van de il6 en tnfa genen zien. Gestimuleerde, getrainde macrofagen lieten verhoogde productie van zuurstof radicalen en stikstof radicalen zien, in vergelijking met gestimuleerde ongetrainde macrofagen. Na de opzet van het model hebben we getoetst of laminarin, een oplosbare $\beta$-glucaan, ook in staat was om trained immunity te induceren in karper macrofagen. Ook na training met laminarin zagen we een duidelijke toename in de productie van zuurstof radicalen in gestimuleerde getrainde macrofagen ten opzichte van gestimuleerde ongetrainde macrofagen.

Nadat we de evolutionaire conservatie van trained immunity in vissen hadden geobserveerd en we profielen gelijkend op trained immunity konden induceren in karper macrofagen met behulp van $\beta$-glucanen, zijn we andere potentiele onderliggende mechanismen van de $\beta$-glucaan geïnduceerde immuun-modulatie gaan onderzoeken. In hoofdstuk 4 hebben we onderzocht of de darm microbiota een rol speelde in de immuun-modulatie na suppletie van het dieet met $\beta$ glucanen. Daartoe hebben we eerst de actieve microbiota van naïeve karpers geanalyseerd met 16S rRNA sequencing. We hebben toen op basis van de aanwezigheid van verschillende genera, de hypothese gesteld dat de microbiota van karpers in staat zou moeten zijn om $\beta$-glucanen te fermenteren. Vervolgens 
hebben we een in vitro fermentatie experiment uitgevoerd waarmee we aantoonden de darm microbiota van karpers in staat is om $\beta$-glucanen te fermenteren en observeerden we specifieke propionaat productie na fermentatie van een van de geanalyseerde $\beta$-glucanen. Daarna hebben we, om de in vivo effecten van $\beta$-glucanen op de darm microbiota en de lokale genexpressie te onderzoeken, karpers een enkele behandeling gegeven met orale $\beta$-glucaan spoeling. Op dag 7 na de behandeling, zagen we een verandering in de actieve microbiota en zagen we een duidelijke regulatie van pro-inflammatoire genen, $i / 1 \beta$, il6 en tnfa in de darm. In het licht van de huidige literatuur op het gebied van de immuun-modulatoire effecten van de metabolieten, geproduceerd door de microbiota na degradatie en fermentatie van voedingsvezels, formuleren we tenslotte de hypothese dat de geobserveerde regulatie in de darm na een $\beta$ glucaan supplement, het gevolg kan zijn van de fermentatie van die $\beta$-glucanen door de microbiota.

Om het laatste mechanisme te onderzoeken wat aan het begin van het project werd geopperd, hebben we eerst de mogelijkheid om gebruik te maken van next generation sequencing (NGS) in relatie tot dit mechanisme onderzocht. In hoofdstuk 5 hebben we de huidige genomen van beenvissen bekeken en hebben we op NGS gebaseerde studies naar vissen immunologie samengevat. Daarnaast hebben we de complexiteiten en mogelijke valkuilen van het gebruik van NGS in polyploïde soorten, zoals de karper, bediscussieerd. Tot slot, bieden we een vooruitblik naar toekomstige technologische ontwikkelingen die een uitkomst kunnen bieden bij genoom analyses van complexe genomen. Vervolgens hebben we in hoofdstuk 6 zelf, met behulp van NGS, een transcriptoom analyse uitgevoerd van macrofagen gestimuleerd met $\beta$-glucanen. Om achtergrond ruis vanuit andere cel typen te minimaliseren hebben we een primaire macrofagen celkweek gebruikt als uitgangspunt. Op basis van differentiële genexpressie profielen, konden we met geautomatiseerde analyses, de regulatie van verschillende signaalcascades identificeren. Daarnaast hebben we met handmatige analyses vast kunnen stellen dat de C-type lectin receptor signaalcascade gereguleerd werd. De regulatie van deze cascade suggereert dat een deel van de respons op $\beta$-glucanen evolutionair geconserveerd is tussen zoogdieren en karpers. Daartoe hebben we de C-type lectin receptoren in het karper genoom geanalyseerd. Op basis van expressie van de genen en de structurele architectuur van hun bijbehorende eiwitten, konden we tot slot een aantal kandidaat receptoren voorstellen.

Tot slot, integreer ik alle voorgaande hoofdstukken in een groter kader en bediscussieer ik de implicaties van mijn bevindingen voor toekomstig onderzoek en voor de toepassing van $\beta$-glucanen in de aquacultuur in hoofdstuk 7. Ik begin met een reflectie op de geobserveerde inductie van trained immunity in karper en bediscussieer overeenkomsten en verschillen tussen onze observaties en de studies in zoogdieren naar trained immunity. Daarnaast bediscussieer ik de potentie maar ook de mogelijke keerzijde van trained immunity voor de aquacultuur sector. Tenslotte stel ik twee mogelijke studies voor die bruikbare 
inzichten kunnen bieden voor zowel de fundamentele en de toegepaste kant van het verhaal. Ik vervolg met een discussie over onze geobserveerde effecten van $\beta$-glucanen op de karper darm microbiota. Naast dat ik onze observaties in een bredere context plaats, bediscussieer ik mogelijke implicaties van de aanwezigheid van fermentatieproducten en $\beta$-glucanen in de darm, in de context van trained immunity. Vervolgens neem ik onze observaties van de gereguleerde C-type lectin receptor signaalcascade een stap verder door onze dataset met nieuwe datasets te vergelijken. Door onze gepubliceerde datasets te vergelijken met transcriptoom datasets van macrofagen gestimuleerd met een andere $\beta$-glucaan, zymosan, of met LPS, als een algemeen inflammatie inducerend ligand, ben ik in staat om een specifieke $\beta$-glucaan gereguleerde genen set te identificeren. Ik vervolg de discussie met het analyseren van een, tot dan toe, nog niet genoemd herkenningsmechanisme van $\beta$-glucanen, de peptidoglycaan herkennings eiwitten (PGRPs), en de mogelijke evolutionaire conservatie van die rol van invertebraten naar vissen. Tenslotte, bediscussieer ik dat er meer vissen in de zee van immuunmodulatoire stoffen zwemmen en bediscussieer ik het gebruik van zogenaamde mariene sulfaat polysachariden als een alternatief op $\beta$-glucanen. Ondanks dat ik gedurende de discussie al reflecteer op de relevantie van onze bevindingen voor de aquacultuur sector, sluit ik af door bewust de korte termijn en mogelijke lange termijn implicaties van mijn observaties voor de aquacultuur sector te behandelen.

De diverse invalshoeken die we hebben genomen voor de studies in dit proefschrift hebben geleid tot een beter begrip van de onderliggende mechanismen die mogelijk de immuun-modulatoire effecten van $\beta$-glucanen op karpers verklaren. Deze verbeterde kennis van de $\beta$-glucaan geïnduceerde immuun-modulatie kan als een sterke basis dienen voor toekomstige studies. Op de langere termijn, kunnen de bevindingen in dit proefschrift mogelijk bijdragen aan het bereiken van concepten als "gepersonaliseerd voeren", vergelijkbaar met "gepersonaliseerde voeding". De gezondheid van vissen zou verbeterd kunnen worden door de optimalisatie van voerstrategieën, wat vervolgens de noodzaak van inzet om andere maatregelen om de gezondheid te verbeteren, zoals antibiotica en vaccinatie, kan verminderen. 


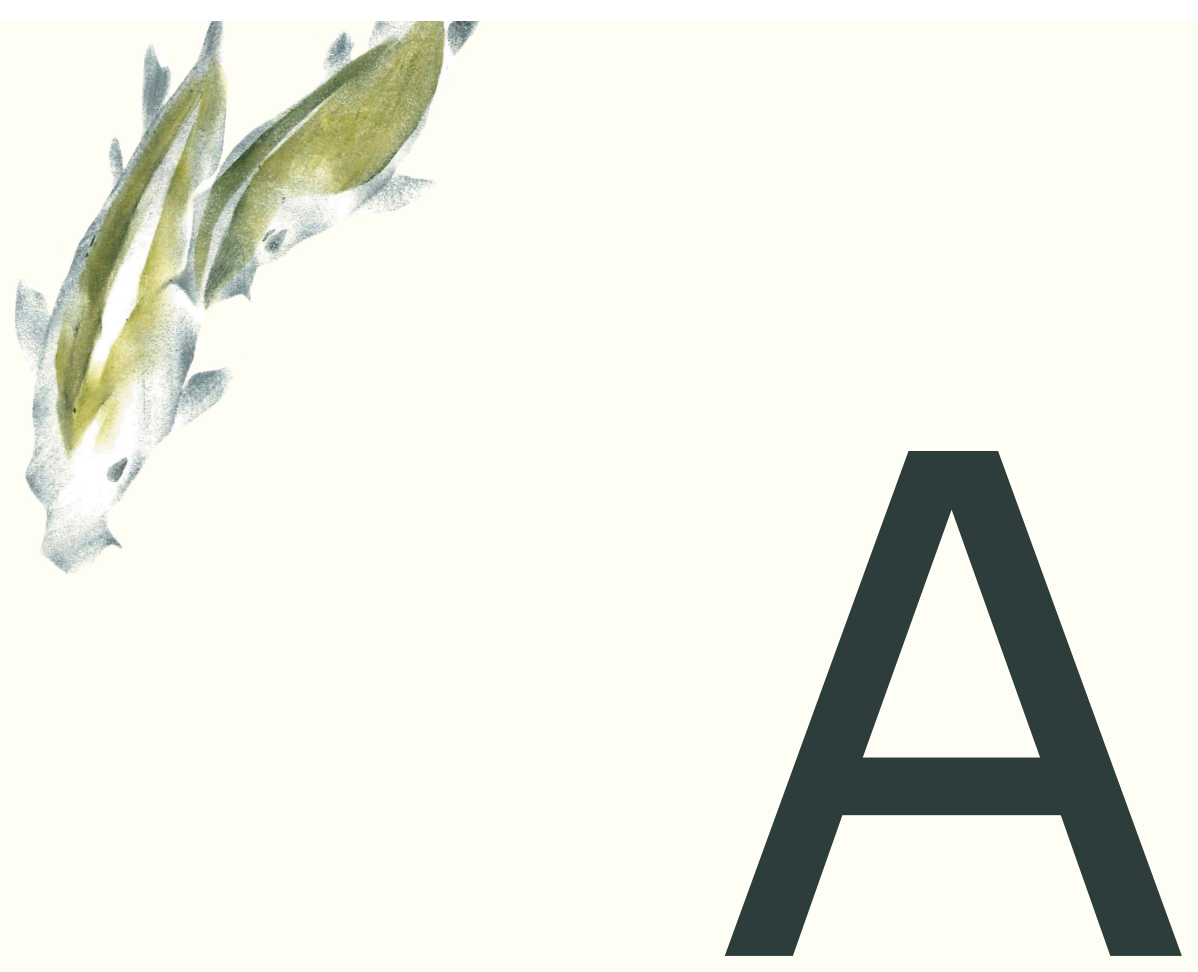

About the author

List of Publications

Overview of completed training activities

Acknowledgements

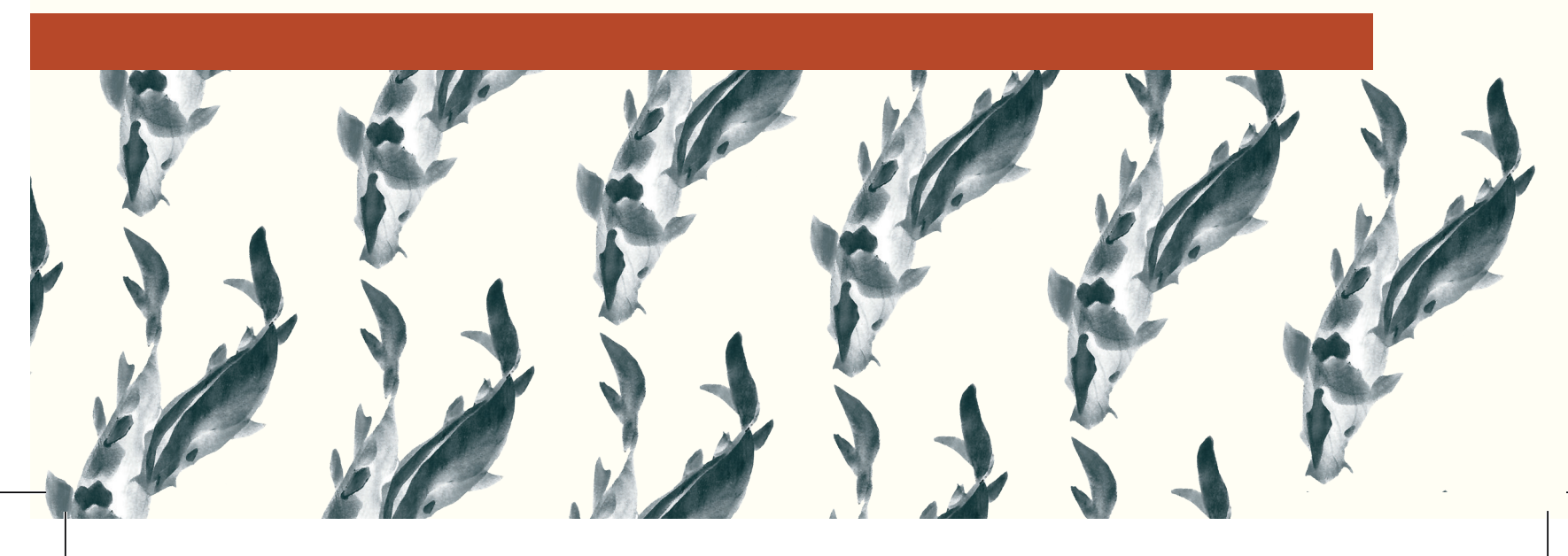




\section{About the author}

Jules Petit was born on the $18^{\text {th }}$ of November, 1989 in Nijmegen. He enrolled in a three-year program for gifted children during the first three years of his high school (Mondriaan College, Oss). After three years, the program ended and Jules enrolled in regular Athenaeum education. From his early days Jules was fascinated by oceans, seas, water and their inhabitants. In 2007, Jules started his BSc Biology at the Wageningen University, with the aim to become a marine ecologist. During his BSc period Jules spent a full year, performing extracurricular activities, as a board member of a student association. During this he was, together with seven other board

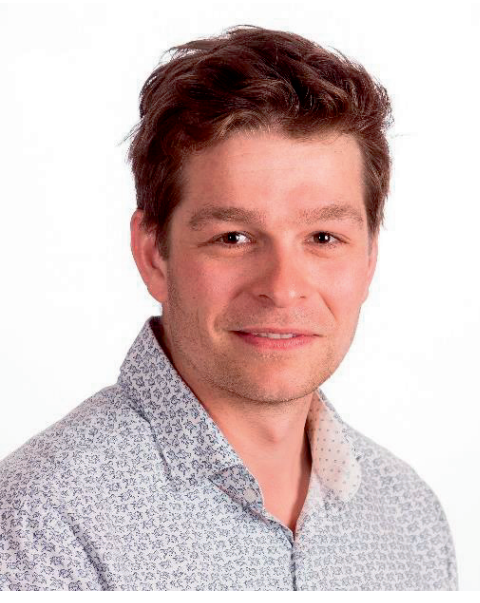
members, responsible for the daily organization of an association with 600 active members.

After Jules finished his BSc, he came to the conclusion that another great interest was in immunology, and therefore continued his MSc studies in Biology with the specialization "Health and Disease". During his major MSc thesis he was able to combine these two passions in the form of independent research on the characterization of carp interleukin-12 and its possible use as an adjuvant during vaccination. He performed this research under the supervision of Dr Maria Forlenza at the Cell Biology and Immunology group of the Wageningen University. He continued his studies during an internship abroad at the Virologie et Immunologie Moléculaires department of the Institut national de la recherche agronomique (INRA) at Jouy-en-Josas, France. Under the supervision of Christelle Langevin and Pierre Boudinot he investigated finTRIMs and their potential role in antiviral responses. Jules obtained his BSc degree in 2012 and his MSc degree in 2014.

In January 2015, Jules started his PhD project at the Cell Biology and Immunology group of the Wageningen University under the supervision of Geert Wiegertjes and Maria Forlenza. His aim was to explain the effects of $\beta$-glucans on carp immune responses. The most important findings of his work are presented in this thesis. During this project there was a strong collaboration with the Brazilian partners reflected by several visits to Brazil and an exchange between labs of four weeks.

While finishing his PhD thesis, Jules already started as a Post-doctoral researcher at the Aquaculture and Fisheries group under the supervision of Geert Wiegertjes and Johan Schrama. In his current position he is investigating the effects of marine sulphated polysaccharides on the immune system of several important aquaculture fish species. 


\section{List of publications}

Petit, J.; de Bruijn, I.; Brugman, S.; Pellikaan, W.F.; Forlenza, M.; Wiegertjes, G.F. $\beta$-glucan immuno-modulation in common carp intestine: a role for microbiota and its metabolites. Manuscript in preparation

Petit, J.; Embregts, C.W.E.; Forlenza, M.; Wiegertjes, G.F. Evidence of trained immunity in teleost fish: conserved features in carp macrophages. The Journal of Immunology, Accepted for publication (2019)

doi: $10.4049 /$ jimmunol.1900137

de Jesus, R.B.; Petit, J.; Pilarski, F.; Wiegertjes, G.F.; Koch, J.F.A.; de Oliveira, C.A.F.; Zanuzzo, F.S. An early $\beta$-glucan bath during embryo development increases larval size of Nile tilapia. Aquaculture Research (2019)

doi: $10.1111 /$ are. 14047

Petit, J.; Bailey, E.C.; Wheeler, R.T.; de Oliveira, C.A.F.; Forlenza, M.; Wiegertjes, G.F. Studies Into $\beta$-Glucan Recognition in Fish Suggests a Key Role for the C-Type Lectin Pathway. Frontiers in immunology 10, article 280 (2019)

doi: $10.3389 /$ fimmu.2019.00280

Embregts, C.W.E.; Rigaudeau, D.; Veselý, T.; Pokorová, D.; Lorenzen, N.; Petit, J.; Houel, A.; Dauber, M.; Schütze, H.; Boudinot, P.; Wiegertjes, G.F.; Forlenza, M. intramuscular Dna Vaccination of Juvenile carp against spring Viremia of carp Virus induces Full Protection and establishes a Virus-specific B and T cell response. Frontiers in immunology 8, article 1340 (2017)

doi: $10.3389 /$ fimmu.2017.01340

Petit, J.; David, L.; Dirks, R.; Wiegertjes, G.F. Genomic and transcriptomic approaches to study immunology in cyprinids: What is next? Developmental \& Comparative Immunology 75, 48-62 (2017)

doi: $10.1016 / j . d c i .2017 .02 .022$

Petit, J.; Wiegertjes, G.F. Long-lived effects of administering $\beta$-glucans: indications for trained immunity in fish. Developmental \& Comparative Immunology 64, 93-102 (2016)

doi: $10.1016 /$ j.dci.2016.03.003 


\section{Overview of completed training activities}

The Basic Package

2 ECTS

WIAS Introduction Day

2015

Research Integrity \& Ethics and Animal Science (RI-EPAS)

2017

\section{Disciplinary Competences}

19 ECTS

Writing a literature review:

"Long-lived effects of administering $\beta$-glucans: Indications for trained immunity in fish

Advanced course immunology UMC

Participant 16th International Fish Immunology Workshop,

Statistics and R for the Life Sciences (EDx, Harvard)

Bioinformatic Methods I (Coursera, University of Toronto)

Bioinformatic Methods II (Coursera, University of Toronto)

Summer Frontiers 2016 - Systems Biology of Innate Immunity

Epigenesis and Epigenetics (VLAG)

Genomics and Bioinformatics Workshop (IMPRESS, Leiden)

Advanced statistics of Life Science (WIAS)

\section{Professional Competences}

Competence Assessment (WIAS)

Participant WPC symposium (WPC)

Employability outside Academia (PCDI)

\section{Presentation Skills}

4 ECTS

Wellcome Trust Innate Immune memory, Cambridge (poster)

The $13^{\text {th }}$ ISDCI conference, Murcia, Spain (poster)

European Macrophage and Dendritic Cell Society (poster)

Epigenesis and Epigenetics course, Wageningen (Oral)

19th International Fish Immunology Workshop (Oral)

WIAS Science Day 2018 - best oral presentation prize (oral)

The $14^{\text {th }}$ ISDCI conference, Santa Fe, USA (Oral)

The 14th ISDCI conference, Santa Fe, USA (Poster) 
Teaching Competences

6 ECTS

MSc cours: "Human and Veterinary Immunology" (4x)

2015-2018

Practical supervision "International Fish Immunology Workshop" (3x) 2016-2018

Supervising major Master thesis (3x)

2015-2018

Supervising minor Master thesis

2017

Review of Research Master Cluster proposals

Presentation on Supervisor-PhD - WIAS Introduction day (3x)

2017-2018

Education and Training total:

32.5 ECTS

Completion of the training activities is in fulfilment of the requirements for the education certificate of the Graduate School of the Wageningen Institute of Animal Sciences (WIAS). One ECTS equals a study load of 28 hours. 


\section{Acknowledgements}

The acknowledgements. Commonly, the last written chapter, but also the first and most well-read chapter. Four years of research are not accomplished on your own and I would like to thank everybody who feels that he or she has contributed in any form to my research or to my physical and mental health. There are some people I would like to thank in particular.

Ten eerste mijn promotor Geert. Zoals beloofd wil ik je graag allereerst bedanken voor alle wetenschappelijke vrijheid die ik heb genoten de afgelopen 4 jaar. Maar zonder gekheid, ik ben je ontzettend dankbaar voor alle hulp, kennis, enthousiasme en ervaring die je met me hebt gedeeld en aan me hebt besteed. Ik heb jouw "open deur" beleid altijd goed gebruikt en kwam bijna dagelijks even langs met een vraag (meestal), een "even checken" (vaak) of een "het lijkt erop dat ik goede resultaten heb, oh nee wacht ik was te voorbarig" (ook regelmatig). Ik waardeer ontzettend dat je altijd even tijd voor me had en nam en ik kijk dan ook erg uit naar de komende twee jaar waarin we onze samenwerking verder kunnen zetten.

Secondly, off course my other promotor Maria. Already during my Master thesis, I got infected with your enthusiasm for science. You were the one that planted a seed in my mind about doing a PhD and look where we are now! Thank you for all your help and advice on experiments. Thank you for every time I had a "short question", which resulted in us spending an hour discussing results, experiments or future opportunities.

Ik wil graag ook alle technicians en studenten bedanken die hebben meegeholpen aan mijn onderzoeksproject. Trudi, Marleen, Ben en Erik, dank jullie wel voor alle hulp en zorgen dat ik in zo'n geweldig lab heb kunnen werken als waar ik in heb kunnen werken. Naast het geweldige lab, ben ik ook dankbaar dat we zo'n goede proeffaciliteit hebben waar ik altijd terecht kon voor nieuwe donoren. Menno, Wian, Sander, Truus en Emily, bedankt voor jullie werk. Cassandra, Mark, Vignesh (volgens mij ben je goed genoeg in Nederlands om dit te snappen) en Koyan, jullie waren allemaal hartstikke goede studenten en ik wil jullie dan ook bedanken voor al jullie inzet en het verrichtte werk tijdens jullie MSc thesis.

My dear collaborators in Brazil, Raphael, Fabio, Carlos and Fabiana, thank you for the pleasant collaboration and the fruitful discussions either in Brazil or in the Netherlands.

Dear (ex) E-wingers, Adrià, Sem, Awatif, Mirelle, Mojtaba, Paulina, Julia, Sandra, Danilo, Adriaan, Christine, Marloes, Esther, Caroline, Lieke, Joeri, Pim, Lana, Julian, Mike, Uros, Kees, Antoine, Pulkit, Wouter, Andres, Myrthe and Sam, thank you for being such great colleagues. It was a pleasure to spend these 4 years with you guys. Thanks for the brilliant PhD-weekends, the drinks and all the laughs! 
Natuurlijk wil ook even stilstaan bij de mensen die me misschien nog wel het meest hebben gezien de afgelopen vier jaar. Olaf, ik liep binnen op kantoor om kennis te maken en het was meteen alsof we elkaar al jaren kende. Dit groeide al snel uit tot een standaard koffieritueel, liefdesodes met behulp van Minnie Riperton en ons liet versmelten tot Olus. Dank voor alle goede gesprekken, slechte koffietjes, goede muziek, slechte muziek, goede adviezen, slechte grappen en je heerlijke nuchtere kijk op het werkende leven! Het ga je goed in Australië! Annelieke, office en macrofagenbuddy. Je kwam wat later in E1264, maar werd eigenlijk direct oneof-the-guys! Fijn hoe we elkaar konden ondersteunen met de macrofagenkweken (waar ik dan weer bijna alles van claimde), het transcriptoom werk en het reilen en zeilen in de "fishunit". Hoewel het wat meer tijd nodig had, ben ik blij dat ik ook jouw muzieksmaak heb kunnen verpesten. Dank je wel voor alles en succes nog even! Je bent er bijna! Carmen, eigenlijk konden we het van begin af aan meteen goed vinden. Dank voor alle hulp, al je enthousiasme, de officeescaperoom, het volledige omtoveren van onze kantoren als er iemand jarig was en natuurlijk voor alle lol die we samen gehad hebben. Ik weet niet wat daar illustratiever voor is, het samen sinaasappels plukken in Murcia om 7 uur 's ochtends of een Fishworkshop borrel eindigen met gasmaskers. Heel veel succes in Rotterdam, je kan het! Mark, net begonnen aan je PhD, maar eigenlijk loop je al heel lang mee. Via je thesis bij mij binnen gerold bij CBI en gewoon net zo lang blijven hangen tot ze je niet meer kwijt konden ;). Dank je wel voor alle koffietjes, de wandelingetjes, het ouwehoeren over muziek, festivals en natuurlijk onze gedeelde passie voor films! Lieve nimfjes van me, Annelieke en Mark, dank jullie wel dat jullie aan mijn zijde staan en de rol van paranimf op je wilde nemen! Dear Paulina, see this as a materialistic way of eternal gratitude, thank you so much for being my interim-paranymph at the last moment!

Tot slot, natuurlijk ook alle overige collega's bij CBI, bedankt voor al jullie steun, hulp, interesse en gezelligheid! To all CBI colleagues, thank you very much for the support, help, interest and fun!

Daarnaast zou ik ook graag de gelegenheid willen aangrijpen om de mensen om me heen te bedanken voor al hun steun, interesse en begrip van de afgelopen vier jaar.

Lieve clubgenoten, dank jullie wel voor jullie interesse de afgelopen jaren. Onze clubreisjes waren altijd een welkome reset button. Fijn om te merken dat we na alweer elf jaar, nog steeds dezelfde slechte humor hebben maar ook nog steeds bij elkaar terecht kunnen voor goede gesprekken. Lieve Lars, allereerst ontzettend bedankt voor je hulp bij de grafische kant van dit proefschrift, zonder jouw creativiteit had dit proefschrift toch aanzienlijk minder glans gehad. Misschien nog wel belangrijker, dank je wel voor alle filmavondjes met heerlijk eten en een hoop gezelligheid. Na Star Wars, alle Tarantino's en de Matrix trilogie wordt het toch echt tijd voor Jurassic Park! 
Lieve oud-huisgenoten van de Burlenburgh, dank jullie wel voor jullie betrokkenheid en interesse de afgelopen vier jaar. De biertjes, slechte grappen en relativering waren af en toe broodnodig en worden dan ook zeer gewaardeerd! Lieve Juri, Hein, Xander en Thomas, bedankt voor alle etentjes, fietstochtjes en welverdiende biertjes!

Lieve senaatsgenootjes, dank voor alle borrels, etentjes en gezelligheid. We zien elkaar misschien niet super frequent, maar als we elkaar zien is het altijd goed. Lieve Coen, dank je wel voor alle sportmomentjes waarbij we allebei even lekker konden ventileren. Fijn om zo snel tussen onderbroekenlol en poep- en plasgrappen te kunnen schakelen naar goede gesprekken, beiden zijn af en toe namelijk heel hard nodig.

Lieve vrienden uit Maasbommel, fijn om te zien dat we na ruim 20 jaar nog steeds contact hebben, samen voetbal kunnen kijken, biertjes kunnen drinken en betrokken bij elkaar zijn. Dank voor jullie interesse en betrokkenheid, de weekendjes weg, een surftripje en natuurlijk alle gezelligheid van de afgelopen jaren. Lieve Rob, dank je wel voor alle belletjes, interesse in mijn onderzoek en je betrokkenheid. Ondanks de crappy carkit maakte dit de ritjes naar werk altijd weer een stuk fijner!

Lieve Aukje en Lucas, bedankt voor de heerlijke etentjes samen, de uitjes en mooie fietstochten en de gezelligheid in Utrecht en Zeist.

Lieve Jules en Dolinda \& Martijn en Moïra, ik ben alweer acht jaar geleden met open armen ontvangen in jullie gezin en ik ben blij en trots dat ik onderdeel van jullie familie mag zijn. Dank jullie wel voor al jullie steun, liefde, betrokkenheid en interesse voor mij en mijn onderzoek!

Lieve Emile en Anouk, met eigenlijk vaste belmomenten (Emile maandagochtend en Anouk dinsdagochtend/middag), hielden we elkaar altijd op de hoogte. Of het nou ging over wat je het weekend had gedaan of over wat je bezig hield, het is fijn om zo'n lieve broer en zus te hebben! Lieve Corinne en Marcel, dank jullie wel voor jullie betrokkenheid en interesse. Het is fijn om te weten dat mijn lieve broer en zus in zulke goede handen zijn!

Lieve papa en mama, door jullie sta ik nu waar ik sta. Jullie hebben me altijd gesteund en stonden altijd voor me klaar. Of het nu met de sleutel voor Ouddorp was of met advies over een huis kopen of over "de leven" an sich. Ik weet dat ik altijd met alles terecht kan bij de Hoogstraat en dat voelt goed! Dank jullie wel voor alles; jullie steun, betrokkenheid, interesse en liefde!

Lieve Tommie, je bent veel te slim, maar ik vraag me af of je dit begrijpt. Dank je wel voor de slapeloze nachten, de rustgevende knuffels en je altijd eigenwijze aanwezigheid. Je bent een echte top kat! 
En tenslotte mijn steun en toeverlaat voor de afgelopen acht jaar, maar eigenlijk al vanaf het begin van mijn studie. Lieve Stefanie, ik ben ontzettend trots op hoe we samen de afgelopen tijd doorgeworsteld hebben. Ik ben blij dat ik mijn leven met jou mag delen en van je steun, je liefde, en (ja ook) je humor mag genieten. Zoals jij ooit al zei, ik ben blij dat je mijn maatje bent! We weten niet wat de toekomst op ons pad werpt, maar met jou durf ik en wil ik dat pad zeker bewandelen! Ik hou van je! 


\section{Colophon}

The research described in this thesis was financially supported the Netherlands Organisation for Scientific Research (NWO) and São Paulo Research Foundation, Brazil (FAPESP) as part of the Joint Research Projects BioBased Economy NWOFAPESP Programme (Project number 729.004.002).

Financial support from Wageningen University and Biorigin for printing this thesis is gratefully acknowledged.

Cover image by Stefanie Bus based on a template from vingerveegkunst.nl Cover design by Lars H.E. Beurskens Lay-out by Lars H.E. Beurskens and Jules Petit

Printed by Proefschrift maken I| Digiforce on FSC-certified paper 